\title{
Hydraulical and acoustical properties of porous sintered glass bead systems: Experiments, theory, \& simulations
}

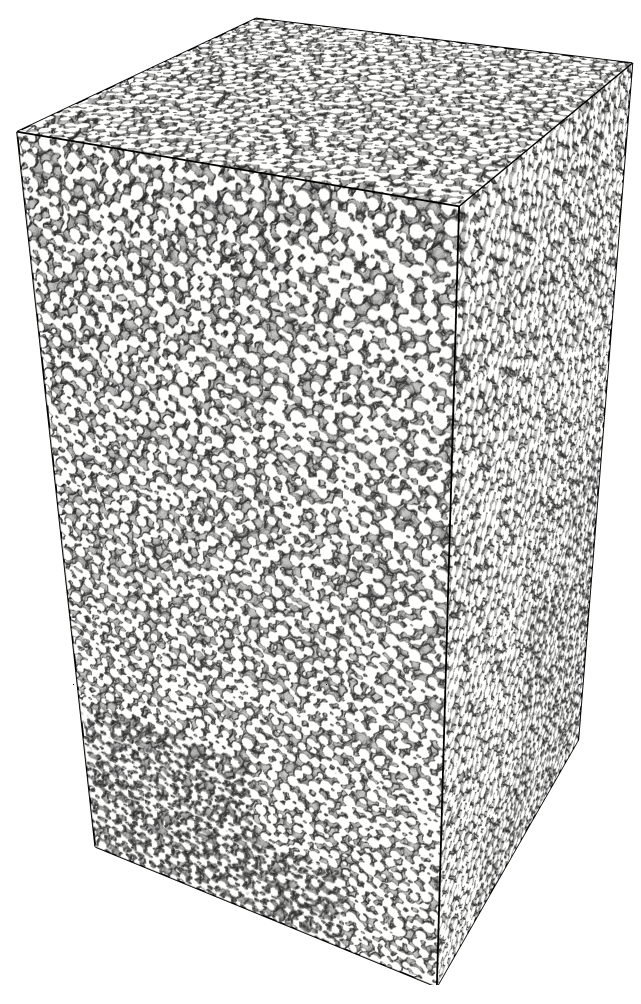

\section{Ibrahim Güven}


Hydraulical and acoustical properties of porous sintered glass bead systems: experiments, theory, \& simulations 
Hydraulical and acoustical properties of porous sintered glass bead systems: experiments, theory and simulations

I. Güven

PhD thesis, University of Twente, the Netherlands

Copyright (C)2016 Ibrahim Güven, Enschede, the Netherlands

All right reserved. No parts of this thesis may be reproduced or transmitted in any form or by any means without the permission of the author or the publishers of the included scientific papers.

ISBN 978-90-365-4135-0

Cover image and design by Ibrahim Güven and Gildeprint

Printed by Gildeprint Drukkerijen - Enschede

The publications of this thesis was supported by: Stichting voor Fundamenteel

Onderzoek der Materie (FOM), which was financially supported by the "Nederlandse Organisatie voor Wetenschappelijk Onderzoek" (NWO), (project number:

09iPOG14-1/2). 


\title{
HYDRAULICAL AND ACOUSTICAL PROPERTIES OF POROUS SINTERED GLASS BEAD SYSTEMS: EXPERIMENTS, THEORY, \& SIMULATIONS
}

\author{
PROEFSCHRIFT
}

Ter verkrijging van

de graad van doctor aan de Universiteit Twente, op gezag van de rector magnificus,

Prof. dr. H. Brinksma,

volgens besluit van het College voor Promoties

in het openbaar te verdedigen

op donderdag 30 june 2016 om 16:45 uur

door

Ibrahim Güven

geboren op 09 december 1983

te Bochum, Duitsland 
Dit proefschrift is goedgekeurd door de promotor:

prof. dr. S. Luding

Samenstelling promotiecommissie:

prof. dr. G.P.M.R. Dewulf

prof. dr. S. Luding

prof. dr. H. Steeb

dr. S. Berg

prof. dr. D. Lohse

prof. dr. T.H. van der Meer

prof. dr. D.M.J. Smeulders

prof. dr. J. Renner
Universiteit Twente, voorzitter/sectretaris

Universiteit Twente, promotor

Universität Stuttgart, co-promotor

Shell Int. Exploration and Production BV.

Universiteit Twente

Universiteit Twente

Technische Universiteit Eindhoven

Ruhr-Universiät Bochum 


\section{Abstract}

Wave and transport phenomena through porous media are of great importance in science and industrial applications, because they involve the interaction of various physical mechanisms and can provide useful informations of the structure of the porous medium. Despite the extensive application in modern industry and comprehensive research, transport and wave propagation through porous materials are not fully understood.

This work focuses on the investigations of hydraulical and acoustical properties of sintered glass bead systems, which serve as replacement for rock samples. In contrast to common rock samples the hydraulical and acoustical properties of the sintered glass bead samples can be selectively influenced by the selection of certain glass beads and sintering treatments. Moreover, their high gray-scale contrast to the pore space, in addition to the relatively simple pore structure favors their use in scientific research and image analysis.

The research goal is to understand the essential physical phenomena and mechanisms, which determine the transport and wave behavior in fluid-saturated porous sintered glass bead systems. For this purpose, a multi-purpose measuring cell is developed, which enables, besides stationary and dynamic permeability measurements, also to perform ultrasound experiments, while the produced porous sintered samples can be saturated with different fluids. The steady-state and oscillatory flow processes through sintered glass bead packings, are investigated, in order to determine both, the stationary and frequency-dependent hydraulic properties. For the ultrasound experiments the porous sintered samples are either saturated with Newtonian fluids, like water or silicone oil, or with magnetorheological fluids (MRF), which is a suspension of micron-sized, magnetizable particles in a silicone-oil-based carrying fluid. The wave propagation in MRFsaturated porous sintered samples is studied at different magnetization strengths. The ultrasound experiments are performed according to the transmission method and analyzed using the spectral ratio technique. The ultrasound experiments reveal nicely the dispersive nature of the fluid-saturated sintered granular materials and show strong frequency filtering as the dispersion properties of the detected waves are highly influenced by the pore fluid. The experimental studies of transport and waves are complemented by extensive research on the morphology by using XRCT-based images of the produced samples. A special focus lies on the elaborate $\mu \mathrm{XRCT}$ data processing of the produced samples. The proposed frameworks for $\mu \mathrm{XRCT}$ analysis can be used for arbitrary porous media. The binarized and differently-sized voxel data are incorporated in Lattice Boltzmann simulations to determine the local permeabilities numerically and finally are compared with experimental effective permeabilities. The numerical permeabilities are correlated with averaged microscopic features, such as pore throats. 


\section{Samenvatting}

Golf- en transportfenomenen in poreuze media zijn van zeer groot belang in de wetenschap en voor industriële applicaties, omdat er zich verscheidende fysische mechanismes afspelen en ze nuttige informatie over de structuur van het poreuze media kunnen geven. Ondanks de vele toepassingen in de moderne industrie en uitgebreid onderzoek zijn de mechanismes achter golf- en transportfenomenen nog niet helemaal begrepen.

Dit werk focust zich op het onderzoeken van hydraulische en akoestische eigenschappen van systemen van gesinterde glazen bolletjes, welke als vervanging dienen van rotsmonsters. In tegenstelling tot standaard rotsmonsters zijn de hydraulische en akoestische eigenschappen van de gesinterde glazen bolletjes selectief te beinvloeden door de materiaalkeuze en het type sinterbehandeling. Bovendien zijn deze systemen practischer in wetenschappelijk onderzoek en beeldanalyses door hoog contrast in grijstinten tussen de glazen bolletjes en de poriÃńn waardoor de porie structuur zeer duidelijk zichtbaar is. Ook is de poriestructuur van systemen met bolletjes eenvoudiger.

Het onderzoeksdoel is het begrijpen van de essentiële fysische fenomenen en mechanismes die het transport- en golfgedrag beïnvloeden in systemen van vloeistofverzadigde poreuze media van gesinterde glazen bolletjes. Om dit te bereiken is een meetcel ontworpen waarmee, naast stationaire en dynamische permeabiliteitsmetingen, het ook mogelijks is om experimenten met ultrageluid uit te voeren en bovendien kunnen de systemen met verschillende type vloeistof worden verzadigd. De constante-toestand en niet-constante vloeiprocessen door verschillende stapelingen van gesinterde glazen bolletjes is onderzocht om te bepalen wat de stationaire en frequentie-afhankelijke hydraulische eigenschappen zijn. Voor de ultrageluid experimenten zijn de poreus gesinterde monsters gesatureerd met of een Newtoniaanse vloeistof zoals water of siloconen olie, of magnetorheologische vloeistoffen (MRF), waarbij de laatste een suspensie is van magnetische deeltjes ter grote van een micron in een op siliconen gebaseerde olie als drager vloeistof. De voortplanting van golven in poreus gesinterede monsters, gesatureerd met MRF vloeistoffen, is onderzocht bij verschillende sterktes van het magnetisch veld. De ultrageluid experimenten zijn uitgevoerd volgens de transmissie methode en geanalyseerd met de spectrale verhouding techniek. De ultrageluid experimenten laten aardig het verspreide gedrag van vloeistof-gesatureerd gesinterde granulaire materials zien en laten sterkte frequentie filtering zien als de verspreidingseigenschappen van de waargenomen golven sterk beïnvloed worden door de vloeistof in de poriën. De experimentele studie van transport en golven zijn aangevuld door uitgebreid onderzoek naar de morfologie met behulp van Röntgenstraling-computer-tomografie (XRCT) beelden van de geproduceerde monsters. Er is special focus gelegd op de uitgebreide micro-Röntgenstraling-computer-tomografie ( $\mu \mathrm{XRCT})$ data ver- 
werking van de gemaakte monsters. De voorgestelde methode voor de $\mu$ XRCT analyse kan worden gebruikt voor arbitrair gevormde poreuze media. De gebinariseerde grijstintbeelden en voxel data, van verschillende grote, zijn toegevoegd aan Lattice Boltzman simulaties om de lokale permeabiliteit numeriek te meten. Deze waardes zijn vergeleken met de effectieve experimentele permeabiliteiten. De numeriek gemeten permeabiliteiten zijn gecorreleerd met gemiddelde microscopische kenmerken zoals de kleinste radius van een porie kanaal. 



\section{Contents}

List of Figures $\quad$ V

$\begin{array}{ll}\text { List of Tables } & \text { XVII }\end{array}$

$\begin{array}{ll}\text { Notation } & \text { XX }\end{array}$

1 Introduction 1

1.1 Motivation ...................... 1

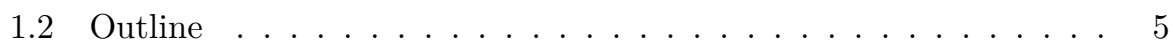

$\begin{array}{llr}2 & \text { Stationary hydraulical properties } & 9\end{array}$

2.1 Introduction . . . . . . . . . . . . . . . . . 10

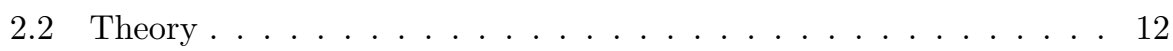

2.3 The lattice Boltzmann method . . . . . . . . . . . . . . . 14

2.4 Experiments . . . . . . . . . . . . . . . . 15

$2.5 \mu \mathrm{XRCT}$ data processing . . . . . . . . . . . . . . . 20

2.6 Results and discussion . . . . . . . . . . . . . . . 25

2.7 Conclusion ......................... 41

2.8 Acknowledgement . . . . . . . . . . . . . . . . 43

3 Dynamic hydraulical experiments 45

3.1 Introduction . . . . . . . . . . . . . . . . . 45

3.2 Theoretical background . . . . . . . . . . . . . 47

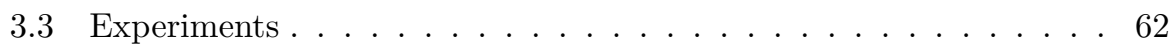


3.4 Conclusion . . . . . . . . . . . . . . . . 83

3.5 Acknowledgement . . . . . . . . . . . . . . 85

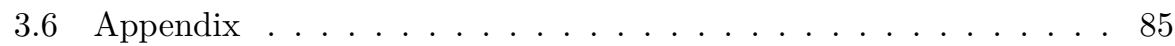

4 Coherent wave part: Fast P1-wave $\quad 89$

4.1 Introduction . . . . . . . . . . . . . . . . . . 90

4.2 Biot theory . . . . . . . . . . . . . . . . 92

4.3 Experimental methods - Application to sintered glass beads . . . . 98

4.4 Experiments . . . . . . . . . . . . . . . . . . . . . . . . . 104

4.5 Results and discussion . . . . . . . . . . . . . . . 115

4.6 Conclusion . . . . . . . . . . . . . . . . . . . 131

4.7 Acknowledgement . . . . . . . . . . . . . . . . . . 132

4.8 Appendix . . . . . . . . . . . . . . . . . . 132

5 Incoherent wave part: Scattering phenomena 135

5.1 Introduction . . . . . . . . . . . . . . . . . . . . . . . 135

5.2 Experiments . . . . . . . . . . . . . . . 136

5.3 Diffusive wave propagation . . . . . . . . . . . . 137

5.4 Conclusion . . . . . . . . . . . . . . . . . . . 142

5.5 Acknowledgement . . . . . . . . . . . . . . . . . 142

6 Evidence of slow P2-wave 143

6.1 Introduction . . . . . . . . . . . . . . . . . . . . 144

6.2 Theoretical background . . . . . . . . . . . . . . 145

6.3 Experiments . . . . . . . . . . . . . . . . . . . . . 148

6.4 Results and discussion . . . . . . . . . . . . . . . 150

6.5 Conclusion . . . . . . . . . . . . . . . 156

6.6 Acknowledgement . . . . . . . . . . . . . . 157

7 Wave propagation in MRF-saturated sintered sample $\quad 159$

7.1 Introduction . . . . . . . . . . . . . . . . . . . . 160

7.2 Background theory and evaluation algorithm . . . . . . . . . 162

7.3 Experiments . . . . . . . . . . . . . . . . . . 165

7.4 Results and discussion . . . . . . . . . . . . . . 169 
7.5 Conclusion . . . . . . . . . . . . . . . . 178

7.6 Acknowledgement . . . . . . . . . . . . . . . . . 178

8 Summary, conclusion and outlook 183

8.1 Summary and conclusion . . . . . . . . . . . . 183

8.2 Outlook . . . . . . . . . . . . . . . . 187

$\begin{array}{lr}\text { Acknowledgement } & 189\end{array}$

$\begin{array}{ll}\text { Bibliography } & 191\end{array}$

$\begin{array}{lr}\text { Curriculum Vitae } & 209\end{array}$ 


\section{List of Figures}

1.1 Examples of granular and porous media (from left to right and top to bottom): corn grains, hazelnuts, foam for household, rock, rice grains, stevia powder, aluminium foam, pharmaceutical tablets, sandy beach, soil. . . . . . . . . . . . . . . . 4

1.2 Topics of study of sintered glass bead packings treated in this study presented in the form of a life cycle, which explain the six chapters. 6

1.3 Different experimental stages with increasing complexity processed in this study. . . . . . . . . . . . . . . . . . 8

2.1 Experimental arrangement for sintering (left) and measured temperature curves at five different furnace zones (right). . . . . . . . 18

2.2 Experimental set up for stationary permeability experiments (left) and detailed construction drawing of the multi-task measuring cell in mode for permeability measurements (right). . . . . . . . . . . 19

2.3 Stepwise increase of flow rate $Q_{z}$ and differential pressure $\Delta p$ caused by viscous fluid flow through the sintered glass beads sample in dependence of measuring time $t$. . . . . . . . . . . 19

2.4 (Left) Computational domain in LB simulations consisting of a porous glass bead sample and the two fluid chambers denoted as in-and output. The fluid is accelerated upwards in the acceleration zone at bottom. (Right) Process flow chart used for extracting influence parameters and quantities determining the hydraulical properties of the sintered glass bead samples from CT-data. . . . . 21 
2.5 Illustration of the applied multistage filtering process using slice images with 1024 x 1024 pixels: A.) Untreated raw $\mu \mathrm{CT}$ scan data B.) after removing bright spots, C.) after applying the "delineate" filter D.) after applying the "median" filter. The corresponding gray value distributions of the images after each filter step are shown below in the respective panels. The vertical axis is logarithmic and gives the probability of occurrence of the gray values, while the horizontal axis represents the gray value of a voxel. . . . 23

2.6 Volume rendering of the 3D initial cuboid with the dimensions $1024 \times 1024 \times 2048$ voxel $^{3}$, whereby the voxel resolution is $16 \mu \mathrm{m}$. The illustration on top-left shows the initial cuboid before segmentation in the raw state A. State B represent the binarized 3D image after segmentation and state $\mathrm{C}$ shows the separated and labeled system used for determination of the particle number distribution. In panel D., the resulting particle number distributions of differently sized subsets taken from different positions of the entire scanned region in comparison with measurement data obtained from laser granulometry are presented. The initial cuboid contains 78,375 particles, while the other distributions are based on less particles as given in the inset. . . . . . . . . . . . . . 26

2.7 (Top) Illustration of main image processing steps for visualization and quantification of pore throat areas in AVIZO 8.0.1. (Bottom) Resulting pore throat areas in a slightly polydisperse sintered glass bead packing and the principle for determining equivalent pore throat diameter is shown below. . . . . . . . . . . . . 27

2.8 Local distribution of numerically determined porosity and permeability values of subsets with edge lengths of 256 voxels within the initial cuboid with dimensions of $1024 \times 1024 \times 2048$ voxel $^{3}$. The conventional representative volume element (RVE) analysis for porosity and permeability are shown below. . . . . . . . . . . 28

2.9 (Left) Local intrinsic permeabilities of different subsets showing particle diameter between 0.4 and $0.6 \mathrm{~mm}$ as a function of porosity. (Right) Normalized permeabilities of differently sized subsets (with maximum edge lengths of $\triangleright-256$ voxel, $\diamond-512$ voxel, - 1024 voxel, $\triangle-2048$ voxel) from LB simulations and predicted values according to Kozeny-Carman in dependence of their porosity values. The blue curve represents the best fit, whereby the Kozeny-Carman constant $c_{1}$ is used as the only fitting parameter. . 
2.10 Numerically and experimentally determined porosity (left) and permeability (right) for sintered glass bead samples from different particle diameters. The experimentally determined permeability results represent the average value of five to eight independent measurements with the error bar representing the standard deviation of the measurements. The numerical results are obtained from subsets of 1024 voxel-sided cubes. The largest deviation between numerical and experimental permeabilities is observed for the glass bead sample with diameter $3.0 \mathrm{~mm}$. In this case, the representativeness of the investigated subset is not sufficient. . .

2.11 (Left) Filter velocity $Q_{z} / A$ as a function of pressure drop $\Delta p / l$ normalized by the real fluid viscosity $\mu^{f R}$. The permeability measurements are repeated 8 times for the sintered glass bead with diameters between 0.4 and $0.8 \mathrm{~mm}$. The almost linear relationship between the filter velocity and the normalized pressure drop confirms the applicability of Darcy's law and the stability of the experimental setup in a broader range of pressure gradients. (Right)Intrinsic permeability in dependence of test duration $t$. The intrinsic permeabilities of the different measurements show an almost constant behavior in dependence on the test duration for different volume fluxes. The small fluctuations are caused by uncertainties of the measuring equipment. For the sake of clarity, only each 150th measuring point in both figures is plotted. . . . . . . . . . . . . 30

2.12 Cross section view of sintered specimen with glass bead diameter between 0.4 and $0.6 \mathrm{~mm}$ : A.) Raw data, B.) after filtering C.) after segmentation of tubes with different mean radii, and D.) porosity in dependence on the mean radial distance of the pipe cross-section from the center for different specimens featuring different glass bead diameters and degrees of polydispersity. . . . . . . . . . . 33

2.13 Method of determining the so-called centroid path tortuosity from binarized 2D slices of the CT image (left). Distribution of tortuosity within the initial cuboid with the dimensions of $1024 \mathrm{x}$ $1024 \times 2200$ voxel $^{3}$. The geometrical tortuosities are determined from cubes with dimensions 256 voxels in each spatial direction and assigned according to their spatial coordinates (left). . . . . . 37

2.14 Centroid path tortuosities $\alpha$ of differently sized subsets taken from the initial cuboid as a function of porosity $\phi_{0}$ (left). Intrinsic permeabilities $k_{z}^{s}$ plotted against the Centroid Path Tortuosity $\alpha$. . 37 
2.15 Local distribution of numerical determined mean sphericity and specific surface values of subsets with edge lengths of 256 voxels within the initial cube. Distributions for sphericity and relative standard deviation of specific surface related to the minimum specific surface obtained from equivalent particle diameter are shown below (red curves). The corresponding cumulative curves are depicted in green. . . . . . . . . . . . . . . . . . 39

2.16 Local spatial distribution of mean pore throat diameter values of subsets with edge lengths of 256 voxels within the initial cuboid (left). Frequency distribution for equivalent mean pore throat diameter $\left\langle d_{p t}\right\rangle$ (red curve) and the corresponding cumulative curve (green curve) (right) . . . . . . . . . . . . . . . . 40

2.17 Porosity (left) and dimensionless permeability (right) in dependence on the mean pore throat diameter normalized to the mean particle diameter. Both, the porosity $\phi_{0}$ and normalized intrinsic permeability $k_{z}^{s} /\left\langle d_{p t}^{2}\right\rangle$, show linear dependency on the normalized mean pore throat diameter $\left\langle d_{p t}\right\rangle /\left\langle d_{p}\right\rangle \ldots \ldots$. . . . . . . . . . 41

3.1 Sketch of different experimental set ups for permeability determination with corresponding pressure curves: A.) Constant pressure difference method, B.) Pressure oscillation method. . . . . . . . . 47

3.2 Theoretical prediction of the dynamic permeabilities of the investigated sintered samples: A.) Absolute value B.) Phase C.) Real part D.) Imaginary part. . . . . . . . . . . . . . . . . . . . . 52

3.3 Theoretical prediction of the dynamic permeabilities of the investigated synthetic samples: A.) Absolute value B.) Phase C.) Real part D.) Imaginary part. . . . . . . . . . . . . . . . . . . 53

3.4 Normalized measured dynamic permeabilities for the sintered sample S1 at different experimental configurations. . . . . . . . . . 56

3.5 Flow chart for the determination of frequency-dependent hydraulical parameters from pore pressure oscillation method.

3.6 Photographs of latex membrane without (A.) and with (B.) a plastic plate, which serves to increase the stiffness of the latex membrane. Photographs from the bottom view of the test table and of the mounted latex membrane inclusive front plate of the upstream reservoir are shown in C.) and D.). The Dynamic Darcy Cell (DDC) with shaker, generating monochromatic waves up to $5 \mathrm{kHz}$ is illustrated in E.) 
3.7 Experimental arrangement for dynamic permeability measurements (A.) and sketch of measuring cell for derivation of determination equation of dynamic permeability at partial saturation of measuring cell (B.). . . . . . . . . . . . . . . . . 6

3.8 Pressure-time signals of the sintered sample $\mathrm{S} 1$ at $0.95 \mathrm{~Hz}$ obtained from piezo-resistive transducers positioned at the up- and downstream reservoir (A.) with corresponding amplitude spectra (B.). Excitation signal for the latex membrane (C.) with corresponding amplitude spectrum (D.). The measuring cell is not completely flooded with distilled water and no piston plate shown in Figure3.6 (B.) is used to increase the stiffness of the latex membrane. . . . . 67

3.9 Pressure-time signals of the sintered sample S1 $0.95 \mathrm{~Hz}$ obtained from piezo-resistive transducers positioned at the up- and downstream reservoir (A.) with corresponding amplitude spectra (B.). Excitation signal for the latex membrane (C.) with corresponding amplitude spectrum (D.). The measuring cell is completely flooded with distilled water and a piston plate shown in Figure 3.6 (A.) is used to increase the stiffness of the latex membrane. . . . . . . 69

3.10 Pressure-time signals of the sintered sample S1 at $37.87 \mathrm{~Hz}$ obtained from piezo-resistive transducers positioned at the up- and downstream reservoir (A.) with corresponding amplitude spectra (B.). Excitation signal for the latex membrane (C.) with corresponding amplitude spectrum (D.). The measuring cell is completely flooded with distilled water and a piston plate shown in Figure3.6 (A.) is used to increase the stiffness of the latex membrane. . . . . . . . . . . . . . . 70

3.11 Pressure-time signals of the sintered sample S4 at 0.95 (A.) and $37.87 \mathrm{~Hz}$ (B.) with corresponding amplitude spectra (B. and D.).

3.12 Pressure-time signals of the sintered sample S5 at 0.95 (A.) and $37.87 \mathrm{~Hz}$ (B.) with corresponding amplitude spectra (B. and D.).

3.13 Illustration of frequency-dependent phase difference through the example of the sintered sample S5: A.) Original phase difference B.) Corrected phase difference. After unwrapping the phase jumps for frequencies greater than $52 \mathrm{~Hz}$ are eliminated. . . . . . . . . 75

3.14 Development of frequency-dependent phase difference and amplitude ration of the synthetic (A. - B.) and sintered sample (C. - D.) within the investigated frequency range of 0.1 till $1000 \mathrm{~Hz}$. . . . 76

3.15 Development of frequency-dependent phase difference and amplitude ration of the synthetic (A. - B.) and sintered sample (C. D.)within the frequency range of 0.2 till $80 \mathrm{~Hz}$, where resonance effects are negligible small. . . . . . . . . . . . . . . . 
3.16 Graphical representation of the analytical solution of the 1-dim diffusion equation for oscillatory flow through an isotropic porous sample in the dimensionless domain of amplitude ratio $A_{r}$ versus phase shift $\Delta \phi$ for: A.) the sintered sample S1 and B.) the synthetic sample D2 consisting of one capillary tube with a diameter $0.75 \mathrm{~mm}$.

3.17 Intrinsic permeabilities (A.-B.), storage capacity (C.-D.) and diffusion coefficient (E.-F.) of the investigated synthetic samples and sintered samples as function of the oscillation frequency. The left panel illustrates the results for the synthetic samples and the right panel for the sintered samples. . . . . . . . . . . . . . 79

3.18 Comparison of permeabilities determined from the pore pressure oscillation test and classical Darcy experiments (or analaytical determined permeability values) for: A.) synthetic samples consisting of capillary and B.) sintered glass bead samples. . . . . . . . . . . 82

3.19 Amplitude sweep tests of sintered sample S1 at different set up configurations. The measuring cell has been either filled completely filled with distilled water (A.-B.) or up to a certain water level (C.-D.). Amplitude ratio (A.,C.) and phase differences (C.,D.) as a function of the amplitude of the excitation signal which is used for oscillation of the latex membrane. . . . . . . . . . . . . . 84

3.21 Pressure-time signals of the sintered sample D2 at 0.95 (A.) and $37.87 \mathrm{~Hz}$ (B.) with corresponding amplitude spectra (B. and D.).

3.20 Pressure-time signals of the sintered sample D1 at 0.95 (A.) and $37.87 \mathrm{~Hz}$ (B.) with corresponding amplitude spectra (B. and D.).

3.22 Pressure-time signals of the synthetic sample D3 (in series connected tubes) at 0.95 (A.) and $37.87 \mathrm{~Hz}$ (B.) with corresponding amplitude spectra (B. and D.)

3.23 Pressure-time signals of the synthetic sample D4 (in parallel connected tubes) at 0.95 (A.) and $37.87 \mathrm{~Hz}$ (B.) with corresponding amplitude spectra (B. and D.). . . . . . . . . . . . . 87

4.1 Valid frequency range (A.) of Biot's theory according to Hughes et al. [87] and simplified illustration of wave modes, which are predicted in the classical bi-phase Biot theory (B.) [140] . . . . . . . 91

4.2 Photographs of investigated sintered glass bead samples with different particle diameters: A.) $\left.d_{p}=0.4-0.6 \mathrm{~mm} \mathrm{B.}\right) d_{p}=1.0$ -

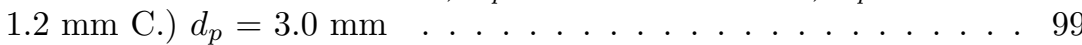


4.3 Extracted volume-rendered cuboid with the dimensions $1024 \mathrm{x}$ 1024 x 2048 voxels of sintered porous sample showing particle diameters between 1.0 and $1.2 \mathrm{~mm}$ (A.). The illustration shows the investigated cuboid after segmentation and labeling procedure. The voxel resolution is $16 \mu \mathrm{m}$. The entire scanned region is demonstrated through the $2 \mathrm{D}$ slice. The corresponding pore space shows a typical foam structure (B.). . . . . . . . . . . . . . . 100

4.4 A.) Visualization of interparticle contact surfaces and neck formation through the examples of glass beads with particle diameters of $d_{p}=1.0-1.2 \mathrm{~mm}$ and $d_{p}=3.0 \mathrm{~mm}$ after the sintering procedure. After idealization of the interparticle contact areas an equivalent neck diameter $d_{n k}$ is determined. B.) Illustration of the main image processing steps for visualization and quantification of inter-particle contact surfaces, which are mainly responsible for the elastic parameters of the solid skeleton composed of sintered glass beads. Note the different scales between Figures (A.) and (B.). . . . . . . 102

4.5 Equivalent neck diameter $d_{n k}$ distribution and corresponding cumulative curves of the investigated samples showing different particle diameter: A.) $\left.d_{p}=0.4-0.6 \mathrm{~mm} \mathrm{B.)} d_{p}=1.0-1.2 \mathrm{~mm} \mathrm{C.}\right) d_{p}=3.0 \mathrm{~mm} .103$

4.6 Time signals of different samples obtained from dry measurements (A.) $d_{p}=0.4-0.6 \mathrm{~mm}$, B.) $d_{p}=1.0-1.2 \mathrm{~mm}$, C.) $\left.d_{p}=3.0 \mathrm{~mm}\right)$ and corresponding normalized power spectra (D.) obtained from the coherent part of the time signals by FFT. The piezo-electric acoustic transmitter is pulsed at the central frequency of $100 \mathrm{kHz}$ using square wave impulser of Panametrics NDT (model 5077 PR). For the comutation of the FFT time windows with approxiamtely 10 to $12 \mu \mathrm{s}$ are used. The samples are directly excited using contact broadband transducers of Panametrics NDT (type V1011). . . . . 105

4.7 A.) Visualization of funnel-shaped pore spaces with distinct pore throats through the examples of glass beads showing particle diameters of $d_{p}=1.0-1.2 \mathrm{~mm}$ and $d_{p}=3.0 \mathrm{~mm}$ after sintering procedure. The pore throats highly influence the coupling between the fluid and solid. B.) Illustration of main image processing steps for visualization and quantification of pore spaces as a result of sintering.106

4.8 Pore volume $V_{\text {pore }}$ distribution and corresponding cumulative curves of the investigated samples showing different particle diameter: A.) $d_{p}=0.4-0.6 \mathrm{~mm} \mathrm{B.)} d_{p}=1.0-1.2 \mathrm{~mm} \mathrm{C}$.) $d_{p}=3.0 \mathrm{~mm}$. . . . . . . . 107

4.9 Experimental arrangements according to the transmission method, whereby the square wave pulser of Olympus (model 5077PR) is used as pulser and pre-amplifier (left) or simply as pre-amplifier (right). . . . . . . . . . . . . . . . . . 109 
4.10 Received time signals, after pre-amplifying with $50 \mathrm{~dB}$, transmitted through the same sample with $d_{p}=1.0-1.2 \mathrm{~mm}$. The piezoelectric transducer is excited with a square wave (left) according to configuration 1 and 1-cycle sinus-burst-signal (right) according to configuration 2 in Figure 4.9. The central frequency of the generated signal is at $1.0 \mathrm{MHz}$. . . . . . . . . . . . . . . 110

4.11 Excitation signals (A.) with corresponding amplitude spectra (B.) at $1 \mathrm{MHz}$ by using configuration 2 in Figure 4.9 (left). . . . . . . . 110

4.12 Simplified sketch of the measuring cell with length specifications used for ultrasound measurements. . . . . . . . . . . . . . . . . 111

4.13 Illustration of principles of Spectral Ratio Technique (SRT): A.) Substitution principle through the use of sintered glass beads B.) Examples of received ultrasound signals recorded in reference medium, such as water (black line) and through water-saturated sintered glass bead sample (red line). C.) Amplitude spectra from the reference signal (black line) and of of the signal transmitted through the water-saturated sample (red line). From the spectral ratio of both signals, the frequency-dependent specific attenuation and phase velocities can be determined. . . . . . . . . . . . . . 114

4.14 Illustration of the frequency-dependent TOFs and specific attenuations of the different waves for the investigated water-saturated sintered samples: The left panel shows the frequency-dependent TOFs (A., C., E.) and the right panel the specific attenuations (B., D., F.) of the different waves. The theoretical predicted curves are determined from dispersion relations formulated in Biot theory using the physical parameters in Table 4.1. . . . . . . . . . . . 116

4.15 Received time signals through water-saturated samples with different glass bead diameters and in water: A.) $d_{p}=0.4-0.6 \mathrm{~mm} \mathrm{B.)}$ $d_{p}=1.0-1.2 \mathrm{~mm} \mathrm{C}$.) $d_{p}=3.0 \mathrm{~mm} \mathrm{D.)} \mathrm{reference} \mathrm{measurement} \mathrm{in} \mathrm{wa-}$ ter. In each measurement, the acoustic transmitter is excited with a 10-cycle-sinus-burst signal at $0.9 \mathrm{MHz}$. The inset at stage D.) shows a zoom of the reference signal in water consisting of 10 sinus cycles. . . . . . . . . . . . . . . . . . . 119

4.16 Received transmission signals through a water-saturated sintered sample showing glass bead diameters between 1.0 and $1.2 \mathrm{~mm}$. The piezo-electric acoustic transmitter is excited with a 10-cycle-sinusburst signal at different frequencies of $0.2 \mathrm{MHz}$ (A.) and $1.0 \mathrm{MHz}$ (B.). . . . . . . . . . . . . . . . . 120 
4.17 Received and windowed time signals of the generated low- (A.) and high-frequency (B.) wave for the sintered sample with $d_{p}=1.0$ $1.2 \mathrm{~mm}$ (shown in Figures 4.16 (A.) and (B.)). The power spectra of the coherent parts of the received signals with the corresponding reference measurement in water are shown in C.). A so-called "Tukey"-window is used to separate the coherent part of the signal from the remaining part. . . . . . . . . . . . . 121

4.18 Normalized power spectra of the investigated samples at different high-frequency (1.0 MHz) excitation signals: A.) 1-cycle-sinusburst B.) 10-cycle-sinus-burst C.) 1-cycle-Ricker-wavelet. For comparison, the corresponding normalized power spectrum obtained from reference measurments in water is also shown. The arrow indicate the increasing particle diameter $d_{p}$ for the peaks. . . . . . 123

4.19 Spectrograms of the different samples and reference measurement, whereby the piezo-electric transmitter is excited with sinus-burstsignal at $1 \mathrm{MHz}$ : A.) $0.4-0.6 \mathrm{~mm}$, B.) $1.0-1.2 \mathrm{~mm}$, C.) $3.0 \mathrm{~mm}$, and the D.) reference measurement (water). Note the different vertical range of for each spectrogram. . . . . . . . . . . . . . 124

4.20 Received time signals through water-saturated samples with different glass bead diameters (using the experimental arrangement depicted in Figure 4.9 A.)): A.) $\left.d_{p}=0.4-0.6 \mathrm{~mm} \mathrm{B.}\right) d_{p}=1.0-1.2 \mathrm{~mm}$ C.) $d_{p}=3.0 \mathrm{~mm}$. Figure D.) shows the peak-normalized Power Spectra of the coherent elastic parts. In each measurement, the acoustic transmitter is briefly stimulated with a square wave pulse

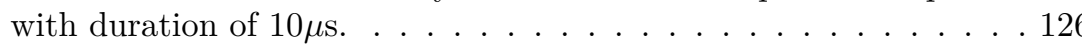

4.21 Comparison of experimentally and theoretically determined phase velocities for the fast $\mathrm{p}$-wave of all investigated samples as function of frequency. The acoustic transmitter is excited with sinus-burstsignals: A.) of 1 cycle B.) of 10 cycles. . . . . . . . . . . . . . . . 127

4.22 Phase velocities $c_{P 1}$, normalized attenuation $\hat{\alpha}$ and specific attenuation $1 / Q_{P 1}$ of the fast $\mathrm{P} 1$-wave of the investigated samples, whereby the piezo-electric transmitter is excited with a 1-cyclesinus-burst-signal at $1 \mathrm{MHz}$. . . . . . . . . . . . . . . . . . . . 128

4.23 Phase velocities of the investigated samples, whereby the piezoelectric transmitter is excited differently with major frequency of $1 \mathrm{MHz}$ : A.) 1-cycle-sinus-burst B.) 10-cycle-sinus-burst C.) 1cycle-Ricker-wavelet. Different symbols correspond to different diameters, while the solid lines give the reference velocity in water. . 129 
4.24 Normalized frequency-dependent attenuation $\alpha_{P 1}$ of the coherent fast P1-wave of the investigated samples at different excitation signals: A.) 1-cycle-sinus-burst signal B.) 10-cycle-sinus-burst signal C.) 1-cycle-Ricker wavelet. . . . . . . . . . . . . . . . . 130

4.25 Received time signals of the generated low- (A.) and high-frequency (B.) wave with corresponding power spectra of the windowed signal. The piezo-electric transmitter is excited with a 1-cycle-sinus burst signal. The power spectra of the coherent parts of received signals with the corresponding reference measurement in water are shown in C.). A so-called Tukey-Win is used to separate the coherent part of the signal from the remaining part. . . . . . . . . . . 133

4.26 Received time signals of the generated low- (A.) and high-frequency (B.) wave. The piezo-electric transmitter is excited with a 1-cycleRicker-wavelet. The power spectra of the coherent parts of received signals with the corresponding reference measurement in water are shown in C.). A so-called Tukey-Win is used to separate the coherent part of the signal from the remainig part. . . . . . . . . . 134

5.1 Received time signal in raw state (A.) and after highpass filtering (B.). The cut-off frequency is chosen at $0.7 \mathrm{MHz}$. The corresponding power spectra of the raw signal (C.) and of the highpass-filtered high-frequency incoherent part are shown below. . . . . . . . . . 138

5.2 Comparison of normalized Intensities obtained from ultrasound measurements and diffusion model. The measured averaged intensity curve is determined from 10 independent measurements. The piezo-electric acoustic transmitter is excited with a 10-cycle-sinus burst at $0.9 \mathrm{MHz}$. The diffusion coefficient and quality factor is determined by fitting the diffusion model the measured averaged intensity profile at $D=0.38 \mathrm{~m}^{2} / \mathrm{s}$ and $Q=105$. . . . . . . . 140

6.1 Simplified sketch of the measuring cell with length specifications used for ultrasound measurements. . . . . . . . . . . . . . . . . . 146

6.2 Frequency-dependent TOFs of the fast P1- (A.) and slow P2-waves (B.) for the investigated porous sintered sample in silicone oil and water. The frequency-dependent TOFs are determined from the dispersion relations according to Equation (6.6). The vertical lines represent the critical frequencies of investigated sample in water and silicone and the gray-shaded area indicates the approximate frequency-range if the received ultrasound signals. . . . . . . . . 147 
6.3 Frequency-dependent wavelength of the fast and slow P-wave in water- and silicone oil-saturated sintered glass bead specimen (A.). The diameter range of the glass particles is represented by the black area. The approximately frequency range of the received time signal is given by the gray-shaded area. The experimental set up for the ultrasound measurements (B.) according to the transmission method. . . . . . . . . . . . . . . . . 150

6.4 Received time signals of the water- and oil-saturated porous sintered sample in raw state (A.-B.) and after lowpass-filtering (C.D.). For comparison the received signals of the reference measurements in water (E.) and silicone oil (F) are shown below. The insets in (E., F.) show an enlargement of the transmitted useful signals determined from the reference measurements. A 1-cyclesinus-burst-signal at $1 \mathrm{MHz}$ is used as electrical input signal. . . . 152

6.5 Received time signals of the water- and oil-saturated porous sintered sample in raw state (A.-B.) and after lowpass-filtering (C.D.). For comparison the received signals of the reference measurements in water (E.) and silicone oil (F) are shown below. The insets in (E., F.) show an enlargement of the transmitted useful signals determined from the reference measurements. A 1-cycleRicker-wavelet at $0.1 \mathrm{MHz}$ is used as electrical input signal. . . . . 154

6.6 Illustration of normalized power spectra (A., C.) and normalized attenuations (B., D.) of the detected waves. The theoretical predictions of the attenuations of the detected waves are represented by solid and dotted lines (B., D.). The piezo-electric transmitter was excited with a 1-cycle-sinus-burst-signal at $1 \mathrm{MHz}$ (A., B.) or a 1-cycle-Ricker wavelet at $0.1 \mathrm{MHz}$ (C., D.). . . . . . . . . 155

7.1 Illustration of MRF behavior, in the cases when no magnetic field is applied (A.) or a magnetic field (B.) is generated [141]. . . . . . 161

7.2 Flow chart for determination dispersion properties (i.e. $\hat{\alpha}(f)$, $Q(f), c(f))$ according to the spectral ratio method. . . . . . . 165

7.3 Photograph of the used porous sintered glass bead sample (A.) and $\mu \mathrm{CT}$ image of the solid matrix (B.) and corresponding pore structure (C.). . . . . . . . . . . . . 166

7.4 Photographs of the used MRF 140CG, when no magnetic field is applied (A.) and a magnetic field is generated by using a NeFeBpermanent magnet (B.). . . . . . . . . . . . . . . . . 167 
7.5 Scaled CAD drawing of the thin-walled measuring cell and the used broadband immersion transducers (central frequency $2.25 \mathrm{MHz}$, Panametrice-NDT, V306-SU). Length informations are given in mm. . . . . . . . . . . . . . . . . . . . . . . . 169

7.6 Photographs of the measuring cell made of acrylic glass and designed to investigate the influence of magnetic strength on the acoustical wave propagation of MRF-saturated porous samples. . . 170

7.7 Received time signals of MRF only (A.) and MRF-saturated sintered glass bead sample (B.). The piezoelectric transmitter is excited with a sinus-burst-signal at $1 \mathrm{MHz}$. The coherent and incoherent parts are marked accordingly. . . . . . . . . . . . . 171

7.8 Examples of raw and windowed signals, when the acoustic transmitter is excited with a sinus-burst-signal at $1 \mathrm{MHz}$. . . . . . . . 172

7.9 Coherent Part of the received time signals (A.,C.) transmitted through only MRF and MRF-saturated sintered sample with corresponding normalized power spectra (B.,D.). The piezo-electric acoustic transmitter is excited with a sinus-burst-signal at $1 \mathrm{MHz} .173$

7.10 Coherent Part of the received time signals (A.,C.) transmitted through only MRF and MRF-saturated sintered sample with corresponding normalized power spectra (B.,D.). The piezo-electric acoustic transmitter is excited with a sinus-burst-signal at $2.25 \mathrm{MHz}$. . . . . . . . . . . . . . . . . . . 175

7.11 Coherent Part of the received time signals (A.,C.) transmitted through only MRF and MRF-saturated sintered sample with corresponding normalized power spectra (B.,D.). The piezo-electric acoustic transmitter is excited with a Ricker wavelet at $0.2 \mathrm{MHz}$.

7.12 Coherent Part of the received time signals (A.,C.) transmitted through only MRF and MRF-saturated sintered sample with corresponding normalized power spectra (B.,D.). The piezo-electric acoustic transmitter is excited with a Ricker wavelet at $1.0 \mathrm{MHz}$.

7.13 Specific attenuation $1 / Q(f)$, attenuation $\alpha(f)$ and phase velocity $c_{P h}(f)$ in dependence on the frequency of the detected fast P1-wave, where different magnetic strength $B$ are applied during ultrasound experiments. The piezo-electric transmitter is excited with a sinus-burst-signal at 0.1 (A.,C.,E.) and $1.0 \mathrm{MHz}$ (B.,D.,F.). 179

7.14 Specific attenuation $1 / Q(f)$, attenuation $\alpha(f)$ and phase velocity $c_{P h}(f)$ in dependence on the frequency of the detected fast P1-wave, where different magnetic strength $B$ are applied during ultrasound experiments. The piezo-electric transmitter is excited with a Ricker wavelet at 0.2 (A.,C.,E.) and $1.0 \mathrm{MHz}$ (B.,D.,F.). . . 180 


\section{List of Tables}

2.1 Material parameters and characteristic particle diameters of the investigated glass beads. . . . . . . . . . . . . . . . 17

2.2 Comparison of characteristic parameters from particle number distributions gained from $\mu \mathrm{m}-\mathrm{CT}$ and laser granulometry. . . . . . . . 24

3.1 Input parameters for the investigated porous sintered glass bead samples. . . . . . . . . . . . . . . . 51

3.2 Input parameters for the investigated artificially produced samples consisting of capillary tubes. . . . . . . . . . . . . . . . . 52

3.3 Comparison of results obtained from analytical solutions and pore pressure oscillation experiments at a specific frequencies. . . . . . 80

3.4 Comparison of results obtained from analytical solutions and pore pressure oscillation experiments at a specific frequencies. . . . . . 81

4.1 Physical parameters of sintered glass beads and water used in Biot model. . . . . . . . . . . . . . . . . . . . . . 108

4.2 Experimental and theoretical determined TOFs and SOSs (Speed Of Sound) of fast P1-wave of investigated sintered samples. . . . . 125

5.1 Input parameters for the diffusion model and results obtained from the fit of the theoretical time-dependent intensity curve to measurement data. . . . . . . . . . . . . . . . . . 141

6.1 Material parameters used for Biot theory. . . . . . . . . . . . . . 149

6.2 Comparison of theoretically and experimentally determined TOFs and SOSs of the slow and fast P-waves. . . . . . . . . . . . . 151

7.1 Material parameters of the used ferro fluid and sintered glass bead sample. . . . . . . . . . . . . . . . . 167

7.2 Material parameters of the used permanent magnets ${ }^{[1]} \ldots$. . . . 170 
7.3 Experimental and theoretical determined TOFs and SOSs of fast P1-wave of investigated sintered samples at a excitation frequency of $1 \mathrm{MHz} \ldots \ldots \ldots$. . . . . . . . . . . . . . . . . 174 



\section{Notation}

\section{Latin characters}

\begin{tabular}{|c|c|}
\hline$f[\mathrm{~Hz}]$ & frequency \\
\hline$f_{\text {crit }}[\mathrm{Hz}]$ & critical frequency \\
\hline$t[\mathrm{~s}]$ & time \\
\hline$c[\mathrm{~m} / \mathrm{s}]$ & speed of sound in bulk material \\
\hline$c(f)[\mathrm{m} / \mathrm{s}]$ & frequency-dependent sound velocity \\
\hline$c_{P 1, P 2, S}[\mathrm{~m} / \mathrm{s}]$ & $\begin{array}{l}\text { frequency-dependent sound velocity of P1-, P2- and S-waves } \\
\text { in the bulk material }\end{array}$ \\
\hline$A_{\text {ref }}(f)[\mathrm{a} . \mathrm{u}]$ & amplitude spectrum of reference signal \\
\hline$A_{0}[\mathrm{a} . \mathrm{u}]$ & instrumentation transfer function \\
\hline$U_{\text {ref }}[\mathrm{a} . \mathrm{u}]$ & $\begin{array}{l}\text { transfer function of ultrasound propagation in reference } \\
\text { medium }\end{array}$ \\
\hline$A(f)[\mathrm{a} . \mathrm{u}]$ & $\begin{array}{l}\text { amplitude spectrum of the received ultrasound signal of the } \\
\text { sample measurement }\end{array}$ \\
\hline$T(f)[-]$ & $\begin{array}{l}\text { product of the transmission coefficient for the reference } \\
\text { material-sample and sample-reference material interfaces }\end{array}$ \\
\hline $\begin{array}{l}U(f)[\mathrm{a} \cdot \mathrm{u}] \\
l[\mathrm{~m}]\end{array}$ & $\begin{array}{l}\text { transfer function of ultrasound propagation in bulk medium } \\
\text { sample length }\end{array}$ \\
\hline$l_{s}[\mathrm{~m}]$ & distance between ultrasound transducer and porous sample \\
\hline$A_{d}(f)[\mathrm{a} . \mathrm{u}]$ & diffraction transfer function \\
\hline$T O F_{\text {ref }}[\mu \mathrm{s}]$ & Time Of Flight of reference measurement \\
\hline$T O F[\mu \mathrm{s}]$ & Time of Flight of sample measurement \\
\hline$\Delta T O F[\mu \mathrm{s}]$ & time difference between sample and reference measurement \\
\hline$\Delta T O F(\omega)[\mu \mathrm{s}]$ & $\begin{array}{l}\text { frequency-dependent time difference between sample and ref- } \\
\text { erence signal }\end{array}$ \\
\hline$G_{P^{d s}, P^{d s}}[\mathrm{a} . \mathrm{u}]$ & $\begin{array}{l}\text { cross power spectrum between pressure signals in the up- and } \\
\text { downstream reservoir }\end{array}$ \\
\hline$E, G, K[\mathrm{GPa}]$ & elastic-, shear- and bulk modulus of skeleton frame \\
\hline$K^{f}, K^{s}[\mathrm{GPa}]$ & bulk modulus of pore fluid and solid grains \\
\hline & Poisson ratio of skeleton frame \\
\hline$k_{z}^{s}\left[\mathrm{~m}^{2}\right]$ & intrinsic permeability of porous sample in flow direction $z$ \\
\hline & particle diameter of glass beads \\
\hline$A\left[\mathrm{~m}^{2}\right]$ & cross-sectional area of the porous sample \\
\hline $\begin{array}{l}\operatorname{grad} p / l[\mathrm{~Pa} / \mathrm{m}] \\
Q_{z}\left[\mathrm{~m}^{3} / \mathrm{s}\right]\end{array}$ & $\begin{array}{l}\text { pressure drop across the porous sample with length } l \\
\text { volumetrical flux in flow direction } z\end{array}$ \\
\hline
\end{tabular}




$\begin{array}{ll}Q(f)[-] & \text { frequency-dependent quality factor } \\ 1 / Q(f)[-] & \text { frequency-dependent specific attenuation } \\ F(f)[-] & \text { frequency-dependent correction factor } \\ b(f)[-] & \text { frequency-dependent viscous damping factor } \\ b_{0}[-] & \text { initial viscous damping factor } \\ b(\omega)[-] & \text { frequency-dependent damping factor } \\ M[-] & \text { shape factor for pores } \\ A, P, N, Q, R[\mathrm{GPa}] & \text { generalized elastic parameters in Biot's theory } \\ \mathbf{A}_{P}, \mathbf{A}_{S}[\mathrm{GPa}] & \text { inertia matrices for P- and S-waves } \\ \mathbf{B}_{P}, \mathbf{B}_{S}[\mathrm{GPa}] & \text { stiffness matrices for P- and S-waves } \\ \hat{\mathbf{\Phi}}, \hat{\mathbf{\Psi}}[-] & \text { potential vectors for longitudinal and transversal } \\ k_{P 1, P 2, S}(\omega), \bar{k}_{P 1, P 2, S}(\omega)[- & \text { frequency-dependent wave number } \\ ] & \\ D\left[\mathrm{~mm}^{2} / \mathrm{s}\right] & \text { frequency-dependent diffusion coefficient }\end{array}$

\section{Greek characters}

$\begin{array}{ll}\omega[1 / \mathrm{s}] & \text { angular frequency } \\ \omega_{c r i t}[1 / \mathrm{s}] & \text { critical ("rollover frequency") } \\ \rho^{f R}\left[\mathrm{~kg} / \mathrm{m}^{3}\right] & \text { real density of saturation fluid } \\ \rho^{s R}\left[\mathrm{~kg} / \mathrm{m}^{3}\right] & \text { real density of grains } \\ \alpha(f)[-] & \text { frequency-dependent attenuation ceoffcient } \\ \hat{\alpha}(f)\left[\mathrm{m}^{-1}\right] & \text { normalized frequency-dependent attenuation ceoffcient } \\ \Delta \phi(f), \Delta \theta(f)[-] & \text { frequency-dependent phase difference between received ultra- } \\ \Delta \phi_{u}(f), \Delta \theta_{u}(f)[-] & \text { sound or pressure signals } \\ & \text { unwrapped phase difference between voltage or pressure sig- } \\ \phi_{r e f}(f)[-] & \text { nals } \\ \phi(f)[-] & \text { phase spectrum of reference signal } \\ \phi_{0}[-] & \text { initial porosity } \\ \phi^{E}[-] & \text { effective porosity } \\ \eta^{f R}, \mu^{f R}[\mathrm{~Pa} \mathrm{~s}] & \text { dynamic viscosity of pore fluid } \\ \delta_{i j}[-] & \text { Kronecka tensor } \\ \rho_{11}, \rho_{12}, \rho_{22}\left[\mathrm{~kg} / \mathrm{m}^{3}\right] & \text { partial densities } \\ \hat{\rho}_{11}, \hat{\rho}_{12}, \hat{\rho}_{22}\left[\mathrm{~kg} / \mathrm{m}^{3}\right] & \text { frequency-dependent partial densities } \\ \delta[-] & \text { frequency-dependent viscous skin depth } \\ \alpha_{\infty}, \alpha[-] & \text { tortuosity of pore channels }\end{array}$




\section{Notation specific to Chapter 2}

\section{Latin characters}

$S_{v}\left[\mathrm{~m}^{2} / \mathrm{m}^{3}\right]$
$f_{i}[-]$
$\bar{d}_{i}[\mathrm{~mm}]$
$r[-]$
$\mathbf{x}[-]$
$\mathbf{c}[\mathrm{m} / \mathrm{s}]$
$t[\mathrm{~s}]$
$n_{z}[-]$
$V_{p}\left[\mathrm{~mm}^{3}\right]$
$A_{p}\left[\mathrm{~mm}^{2}\right]$
$T\left[{ }^{\circ} \mathrm{C}\right]$
$A_{p t}\left[\mathrm{~mm}^{2}\right]$
$\left\langle d_{p t}\right\rangle[\mu \mathrm{m}]$
$\left\langle d_{p}\right\rangle[\mathrm{mm}]$
$c_{1}[-]$

Greek characters volume-based specific surface area fraction of particles between two sieve sizes averaged diameter of glass particles between two sieve sizes shape factor position vector of collision particle velocity vector of collision particle time total number of lattice units in $z$ direction voxel-based volume of glass particle voxel-based surface area of glass particle temperature voxel-based pore throat diameter voxel-based mean pore throat diameter voxel-based mean particle diameter empirical constant in Kozeny-Carman model $\tau[-]$

$\tau_{\text {bulk }}[-]$

$\rho_{s}\left[\mathrm{~kg} / \mathrm{m}^{3}\right]$

$\rho_{b}\left[\mathrm{~kg} / \mathrm{m}^{3}\right]$

$\Delta \rho^{f R}\left[\mathrm{~kg} / \mathrm{m}^{3}\right]$

$\psi^{s}[-]$

$\sigma_{d_{p}}[\mathrm{~mm}]$ relaxation time in $\mathrm{BGK}$ collision model

relaxation time of energy

specific density of glass beads

bulk density of sintered sample

bulk density difference of pore fluid between in- and outlet sphericity of particle

standard deviation of particle diameter 


\section{Notation specific to Chapter 3}

\section{Latin characters}

$\mathbf{w}_{f}[\mathrm{~m} / \mathrm{s}]$

$\mathbf{v}_{f}[\mathrm{~m} / \mathrm{s}]$

$J_{0}, J_{1}[-]$

$K[-]$

$\hat{Q}[\mathrm{~m} / \mathrm{s}]$

$r_{t}[\mathrm{~m}]$

$P^{u s}, P^{d s}[$ a.u. $]$

$V^{u s}, V^{d s}\left[\mathrm{~m}^{3}\right]$

$A_{r}[-]$

$s_{c}\left[\mathrm{~m}^{3} / \mathrm{Pa}\right]$

$s_{c}^{d s}\left[\mathrm{~m}^{3} / \mathrm{Pa}\right]$ relative velocity vector between fluid and solid phase

seepage velocity vector

Bessel functions of zero and first order

shear wave vector

frequency-dependent volumetrical flux

tube radius of pore channel

complex-valued measured pressure transducer

volume of up- down stream reservoir

amplitude ratio between measured pressures in the up- and downstream reservoir storage capacity of porous medium

storage capacity of downstream reservoir

\section{Greek characters}
$\Lambda[\mathrm{m}]$
pore volume to surface ratio
$\delta_{A}[-]$
ratio from reduction of cross-sectional area
$\xi[-]$
dimensionless storage parameter
$\eta[-]$
dimensionless transport parameter 


\section{Notation specific to Chapter 4}

\section{Latin characters}

$$
\begin{aligned}
& \mathbf{u}_{s}, \mathbf{u}_{f}[\mathrm{~m}] \\
& T O F_{f r}[\mu \mathrm{s}] \\
& K_{f r}, E_{f r}[\mathrm{GPa}] \\
& t_{s d}[\mathrm{~h}] \\
& \left\langle d_{n k}\right\rangle[\mathrm{mm}] \\
& T^{f}\left[{ }^{\circ} \mathrm{C}\right]
\end{aligned}
$$

solid and fluid displacement

arrival time of sound wave of the porous sample in dry ultrasound measurements

bulk and elastic modulus of the solid frame determined from dry ultrasound measurements sinter duration voxel-based mean neck diameter between particles fluid temperature

\section{Notation specific to Chapter 5}

\section{Latin characters}

$$
\begin{aligned}
& c_{e}[\mathrm{~m} / \mathrm{s}] \\
& l^{*}[\mathrm{~mm}] \\
& U[\text { a.u. }] \\
& P[\mathrm{a} . \mathrm{u}] \\
& I(t)\left[\mathrm{V}^{2}\right] \\
& U_{0}\left[\mathrm{~V}^{2}\right]
\end{aligned}
$$

ballistic transport velocity transport mean free path for ultrasonic wave spectral energy density of ultrasonic wave field spectral source energy density time-dependent averaged intensity profile of ultrasonic wave field

averaged energy of transmitted scattered wave field 


\section{Greek characters}
$\sigma[\mathrm{a} . \mathrm{u}]$
dissipation rate for spectral energy density of ultrasonic wave
$\tau_{\alpha}[\mathrm{s}]$ field
in elastic absorption time of scattered wave field

\section{Notation specific to Chapter 6}

\section{Latin characters}
$f_{v}[\mathrm{~Hz}]$
viscous frequency
$d_{p} t[\mu \mathrm{m}]$
characteristic pore size (for example pore throat diameter)

\section{Greek characters}

$\zeta[-] \quad$ empirical determined value for assessment of "viscous" frequency

\section{Notation specific to Chapter 7}

\section{Latin characters}

$B[\mathrm{~T}]$

TOF sample $[\mu \mathrm{s}]$

TOF $F^{r e f}[\mu \mathrm{s}]$ residual magnetic strength of the $\mathrm{NeFeB}$ magnet

Time Of Flight of MRF-saturated sample

Time Of Flight of the reference measurement only in MRF 



\section{CHAPTER 1}

\section{Introduction}

\subsection{Motivation}

The term "granular matter" is the general name for materials consisting of grainlike materials, which are acting collectively as an ensemble. They are present in many industrial applications as well as in our daily life, for instance in pharmaceutics or food products like tablets or nuts, cf. Figure 1.1. In general, particle systems with particle sizes larger than $1 \mu \mathrm{m}$ are considered, in which the Brownian motion (thermal agitation) of the particles can be neglected. The grains can exhibit a solid-like or fluid-like behavior, whereby the description of these states and their co-existence is very controversial. In this thesis we consider static, solid porous materials, in which the grain ensemble can be considered as a porous rock model. The particles are held together by solid bridges formed due to sintering. Compared to common rock samples, the homogeneity of the pore space and shape, in addition to the chemical stability and inertness of the sintered glass bead packings are important properties, which promote and facilitate their application in many cases. Moreover, important bulk parameters, such as the porosity, the tortuosity of the pore channels, the stiffness of the solid frame, as well as the glass particle sizes can be adjusted by the selection of certain glass bead sizes and special sintering treatments. Another important characteristic of sintered glass bead systems is the good gray-scale contrast between the pore space and the onecomponent solid frame, which certainly simplifies X-ray based image techniques and data processing.

The porous matrix consisting of sintered glass beads can now be saturated with different pore fluids. Besides the saturation with Newtonian fluids, such as silicone oil and water, non-Newtonian suspensions with magnetizable micron-sized particles suspended in a silicone oil-based carrying fluid are used to fill the pore 
space of the porous sintered system.

In general, fluid-filled porous media are ubiquitous in many natural and industrial systems. Examples of natural fluid-filled porous media and corresponding processes are the water storage in soil or the oil, gas and water flow through porous petroleum reservoirs. Furthermore, the characterization of biological porous materials through the use of ultrasound, such as the clinical assessment of the bone status for the diagnosis of osteoporosis, which is a skeletal disease, is important for medical applications [107]. In this specific case the porous bone matrix is filled with bone marrow. Examples for industrial applications and corresponding processes are the water and gas management in fuel cells or the water and solution movement in building materials [16].

While the fluid-saturated porous sintered glass bead packings are major subject of this thesis, their transport and acoustical wave properties are the physical phenomena to be studied in this work. Both properties are highly affected by the micro- and macroscopic features of the sintered system. In addition, the acoustical properties of the sintered porous bead package is highly affected by the pore fluid, which saturates the pore space of the sintered sample. For instance, the wave speed and the attenuation in sintered glass bead packings strongly depends on the physical parameters of the used saturation fluid and its interaction with the solid matrix. Understanding the above-mentioned transport and wave propagation phenomena in sintered glass bead systems, experimental and numerical studies, and modeling them at different scales especially as a necessity of the spatial heterogeneity, are the subject of this thesis.

The key parameter in the research of porous media, describing the transport capacity of a porous medium, is besides the porosity the intrinsic permeability, which depends only on the geometric characteristics of the porous medium. It is a measure of the ability of the porous medium to transmit fluid mass. We consider not only experimentally and numerically the intrinsic permeabilities of porous sintered glass bead systems from stationary steady-state flow processes, but also their frequency dependence, when an oscillatory fluid movement is induced.

Starting from the generation of simple continuous low-frequency waves $(f<$ $1000 \mathrm{~Hz}$ ) for the study of the frequency dependence of the dynamic permeability, our wave propagation investigations are extended towards short-time, highfrequency broadband ultrasound signals in the range from approximately $100 \mathrm{kHz}$ to $5 \mathrm{MHz}$. The term "ultrasonic" applied to sound refers to anything above the frequencies of audible sound at the limit for human hearing that is approximately at $20 \mathrm{kHz}$.

In general, waves can be encountered directly and have a widespread application in our daily life. We can distinguish between waves that we directly perceive as waves, like the waves in the ocean or the vibration during earthquakes, and waves 
that we perceive without recognizing them as such, like the light we see or the sound we hear. In fact waves have applications in almost every field of everyday life, from wireless communication to radio waves, from the music of a guitar to lasers etc.. Waves are involved in almost every aspect of our daily life. They can appear in a variety of forms, for instance as longitudinal, transversal or rotational waves, surface or body waves etc.. Moreover, they can propagate in various media, such as mechanical waves, or do not need a carrying medium, such as electromagnetic waves. We often distinguish between monochromatic waves, which propagate with a particular frequency and wavelength, such as laser light, and polychromatic waves, which contain more than one wavelength and frequency, for example sunlight.

Consequently, the wide range of applications of wave propagation, due to their special and influenceable properties, involves almost all natural science and engineering disciplines. Wave propagation is central for various engineering disciplines, especially in geophysics and petroleum engineering, and major applications include the transmission, reflection and stimulation through to non-destructive inspection of components. In this respect, the interpretation and prediction of wave propagation is crucial. The experimental study of wave propagation through fluid-saturated sintered glass bead systems, where the determining parameters can be selectively changed, serve to understand the relevant physical processes and to develop appropriate physical models.

The combination of the system of fluid-saturated porous sintered glass bead samples with the propagation of ultrasound yields a versatile research area and opens up new interesting application possibilities.

The characterization of porous media by means of wave propagation, for instance through ultrasound, is very important and has a long history $[14,85,96,98,100$, 106, 144, 167, 176, 186, 189]. Fundamental contributions for the understanding of wave propagation in fluid-saturated porous media were made during the last century $[2,24,25,57,61,94,105,189,194]$. However, the wave propagation in porous media saturated with complex fluids, like magneto-rheological fluids, which change their flow behavior when a magnetic field is applied, has been barely investigated and can open new application fields, for instance in petroleum industry.

Moreover, the complex structure of porous materials can be challenging, because detailed information of their structure is often missing and if available, there is often a lack of appropriate data processing tools.

In recent years significant improvements in imaging techniques and computational performance enabled more comprehensive and precise studies of the structure of 


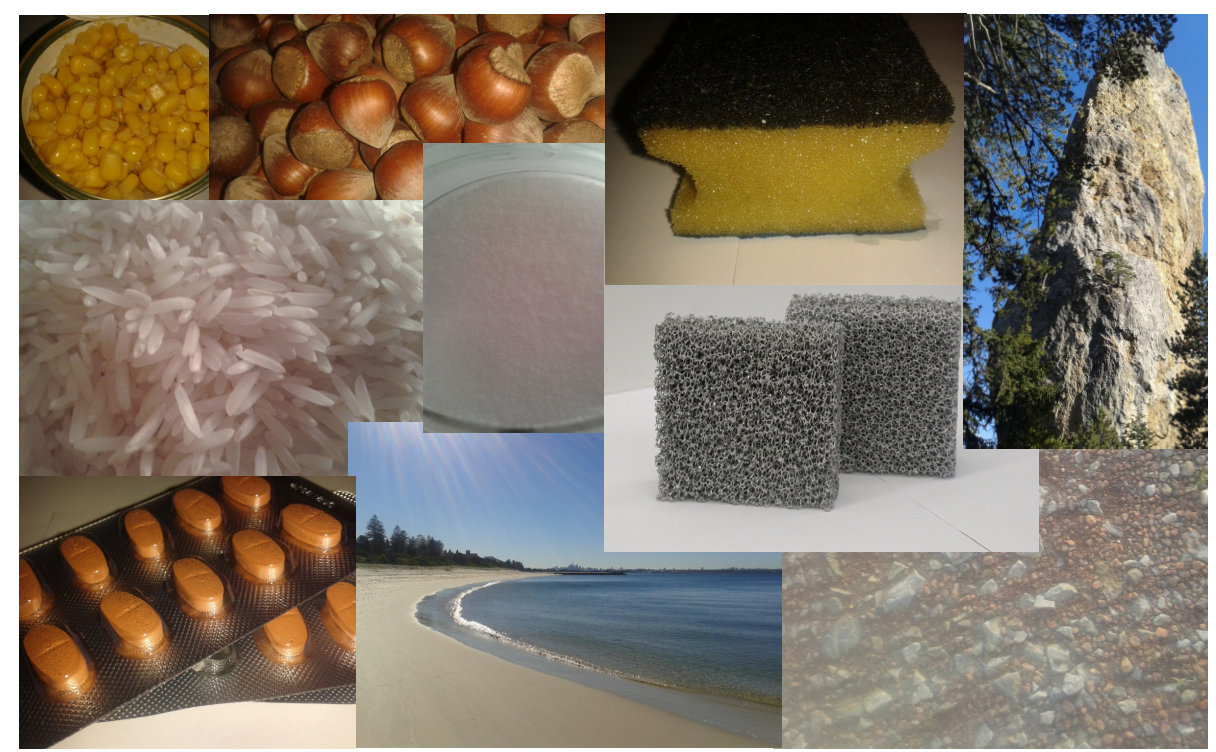

Figure 1.1: Examples of granular and porous media (from left to right and top to bottom): corn grains, hazelnuts, foam for household, rock, rice grains, stevia powder, aluminium foam, pharmaceutical tablets, sandy beach, soil.

porous materials. More detailed information on structural properties, which determine the transport and wave propagation phenomena, has become available. This study utilizes the advantages of XRCT-imaging to characterize the structure of the sintered glass bead packings at different length scales and to understand the pore fluid transport and wave propagation in such sintered granular systems. The relatively simple and repeatable pore structure of the sintered glass bead packings, in addition to the high contrast between the pore space and solid matrix significantly simplifies the image processing and thus increases the understanding of pore fluid transport or wave propagation phenomena in porous media. The XRCT images are embedded in a massively parallel Lattice Boltzmann (LB) simulation framework to determine the intrinsic permeabilities of the investigated randomly distributed system of sintered glass particles [75, 118, 119, 128, 129, 170]. The benefits of understanding the frequency-dependent fluid transport and wave propagation is not only of scientific interest, but also serves to develop purposeful designs in engineering and help to interpret environmental events and processes. 


\subsection{Outline}

The present thesis deals with the hydraulical and the acoustical properties of porous sintered glass bead packings. It aims to

- Understand the relevant processes in transport and wave propagation phenomena in sintered glass bead systems. Specific questions are: Which parameters in sintered granular systems have the strongest impact on the permeability? What is the origin of scattering and frequency-dependent attenuation in ultrasound experiments? How are the dispersion and filtering properties affected by the microstructure of sintered specimens?, What is the frequency response for permeability and for the dispersion quantities? etc..

- Compare numerically and experimentally determined intrinsic permeabilities as result of sintering, giving rise to specific questions as: How do different up- and downscaling processes influence not only the permeability but also the porosity? How do the permeability change with varying frequency, when oscillatory fluid flow is induced?, etc..

- Predict the transport and wave behavior, which involves open issues as: How do the pore fluid influence the wave propagation in sintered glass bead systems? Which parameter in fluid-saturated sintered granular systems support the observation of the slow P2-wave in ultrasound experiments? Is Kozeny-Carman sufficient to predict the permeability in sintered glass bead systems?, etc..

- Interpret and integrate the observations from permeability and ultrasound experiments and answer open questions: How do the generation of an external magnetic field influence the wave propagation in MRF-saturated porous media? Which factors are decisive for the occurrence of scattering phenomena in ultrasound experiments and why? How is scattering related to the microstructure of sintered sample?, etc..

The structure of the thesis is related to the research line and results from publications (submitted and in preparation). Consequently, each chapter is selfcontained and the benefit of this for the reader is only slightly off-set by some overlap of the content. Although different topics are treated, some research methods and theoretical background often are the same, which lead to some more overlap of content.

As denoted in Figure 1.2, sintered glass bead packings are the major focus of our investigations. Each chapter is implemented in a logical order, where different topics are treated by appropriate methods with respect to the involved physical 


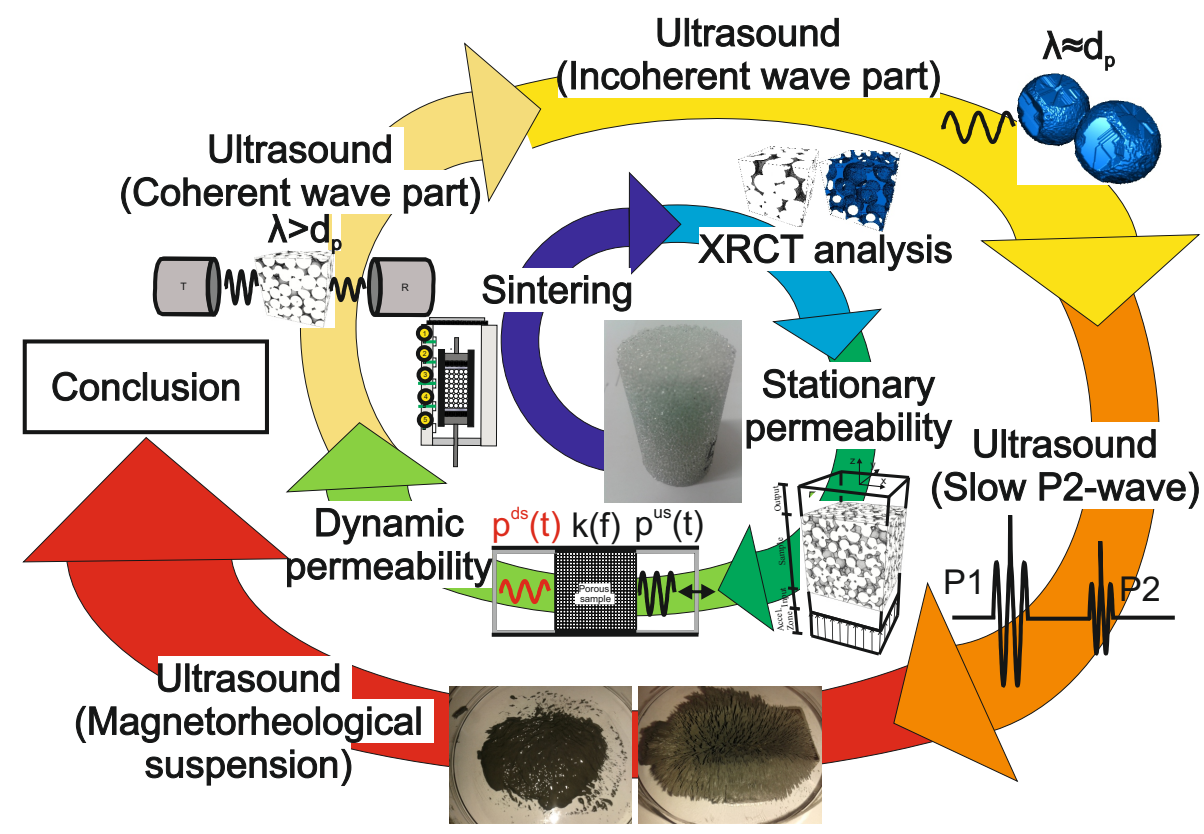

Figure 1.2: Topics of study of sintered glass bead packings treated in this study presented in the form of a life cycle, which explain the six chapters.

processes. As can be seen in Figure 1.2, the entire work can intellectually be divided in nine parts. In addition to this introduction chapter, the final conclusion and the appendix at the end of this thesis, six chapters constitute the core of this work. Depending on the thematic focus each chapter consists of a specific motivation or introduction, respectively, followed by theoretical background, methods and procedures, results, discussion and conclusion.

- The second chapter which follows this first introductory chapter and brings various topics together and relates the hydraulical properties of the porous sintered packings to the sintering procedure, via morphological characteristics from $\mu \mathrm{CT}$ data. A special focus lies on the $\mu \mathrm{CT}$ data processing. Possible ways are presented to extract informations and determine features of sintered granular systems from $\mu \mathrm{CT}$ data, which affect not only the intrinsic permeability, but also the acoustical properties. Differently sized binarized $\mu \mathrm{CT}$ data are used to determine the numerical permeabilities. Those are compared with experimental results and complemented by a comprehensive 
RVE analysis for permeability and porosity.

- In the third chapter the hydraulical properties of the sintered samples are tested for their frequency dependence. The dynamic Darcy cell is presented, where the oscillatory fluid movement through the samples is investigated. The so-called pore pressure oscillation method is introduced and applied to the produced highly permeable sintered samples in order to determine the frequency-dependence not only of the permeability, but also of the storage capacity and diffusivity.

- The fourth chapter deals with the acoustical properties of different porous sintered samples, saturated with water. Focus is on the coherent wave part, which arrives at the leading edge of received signals and shows wavelength greater than the bead diameter $\left(\lambda>d_{p}\right)$. The spectral ratio technique is introduced and used to determine the dispersion properties of the sintered samples with different microstructures. The ultrasound measurements are compared with the classical biphase Biot-theory. $\mu \mathrm{CT}$ scans are again used to extract important parameters determining the wave propagation in fluidsaturated sintered glass bead systems and used as input for the Biot theory.

- In the following fifth chapter the incoherent wave part of the received ultrasound signals is considered. This often consists of (multiple-) scattered waves with wavelengths smaller than the particle sizes $\left(\lambda<d_{p}\right)$. The diffusive wave propagation is introduced and used to describe and understand the scattering phenomena in fluid-saturated porous sintered glass bead samples.

- The sixth chapter specifically addresses the detection and evidence of the slow P2-wave in fluid-saturated porous sintered glass bead packings. For this purpose ultrasound measurements with different pore fluids are performed and compared with each other. The Biot theory is used to identify the detected waves. The difficulties associated with the detection of slow P2-waves in fluid-saturated in sintered glass bead packings are highlighted and discussed.

- The seventh chapter constitutes the investigation of wave propagation of porous sintered samples, saturated with magneto-rheological fluids (MRF). The wave propagation of MRF-saturated sintered samples are investigated at different magnetic strengths and compared with measurements where no magnetic field is applied. The dispersion properties are determined at different magnetic strengths. 


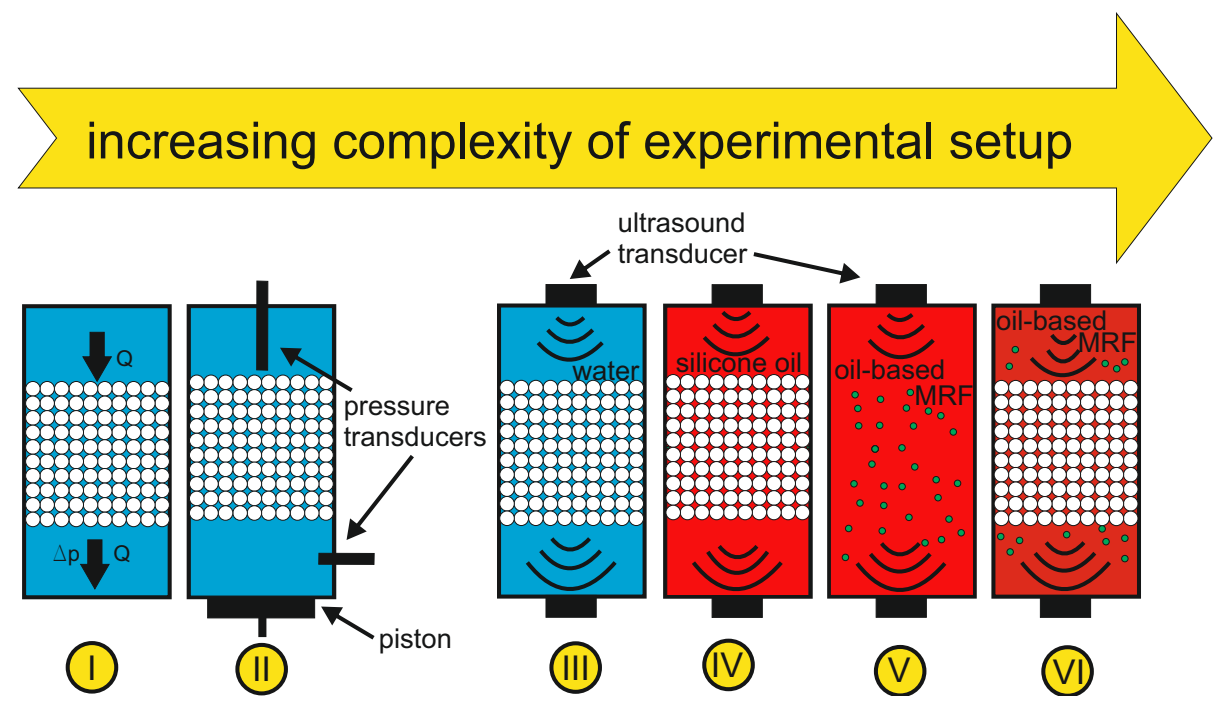

Figure 1.3: Different experimental stages with increasing complexity processed in this study.

- In the eighth chapter the previous chapters are finally reviewed and summarized in a wider, more general framework. A preview for further complementary work and for future research is proposed.

The experimental investigations constitute an important foundation of this work. Figure 1.3 gives an overview of the experimental investigations carried out in this study. It shows simplified sketches of experimental set-ups at different stages, whereby the complexity of the investigated system and the used methods is increasing from left to right in the direction of the arrow. As can be seen in Figure 1.3 the experimental part of this work can be divided in two sections, namely the hydraulical and the acoustical part. The hydraulical part is described by the first two stages and includes the stationary and oscillatory fluid movement through the sintered samples. The acoustical part is represented by the third stage to last stage, whereby different systems and topics are examined using the transmission method. 


\section{CHAPTER 2}

\section{Hydraulic Properties of Porous Sintered Glass Bead Systems}

This article, with the original title "Hydraulical properties porous sintered glass bead systems" by Ibrahim Güven, Stefan Frijters, Jens Harting, Stefan Luding and Holger Steeb, has been submitted to the Granular Matter Journal [69].

In this paper, porous sintered glass bead packings are studied, using X-ray Computed Tomography (XRCT) images at $16 \mu \mathrm{m}$ voxel resolution, to obtain not only the porosity field, but also other properties like tortuosity, particle sizes, pore throat, particle sphericity, specific surface area and the permeability. The influence of the sintering procedure and the original particle size distributions on the microstructure, and thus on the hydraulical properties, is analyzed in detail. The $X R C T$ data are visualized and studied by advanced image filtering and analysis algorithms on to the extracted sub-systems (cubes of different sizes) to determine the correlations between the microstructure and the measured macroscopic hydraulic parameters. Since accurate permeability measurements are not simple, special focus lies on the experimental set up and procedure, for which a new innovative multi-purpose cell based on a modular concept is presented. Furthermore, segmented voxel-based images (defining the microstructure) are used for $3 D$ (three-dimensional) lattice Boltzmann simulations to directly compute some of the properties in the creeping flow regime. A very good agreement between experimental and numerical porosity and permeability could be achieved, validating the numerical model and results. Porosity and permeability gradients along the 
sample height could be related to gravity acting during sintering. Furthermore, porosity increases in the outer zones of the samples due to the different contact geometry between the beads and the confining cylinder wall during sintering (which is replaced by a membrane during permeability testing to close these pores at the surface of the sample).

The influence of different filters on the gray scale distributions and the impact of the segmentation procedure on porosity and permeability is systematically studied. The complex relationships and dependencies between numerical determined permeabilities and hydraulical influence parameters are investigated carefully. In accordance to the well-known Kozeny-Carman model, a similar trend for local permeability values in dependance on porosity and particle diameter is obtained. From the $\mu X R C T$ analysis two distinct peaks in pore throat distributions could be identified, which can be clearly assigned to typical pore throat areas occurring in slightly polydisperse granular systems. Moreover, a linear dependency between average pore throat diameter and porosity as well as between permeability and pore throat diameter is reported. Furthermore, almost identical mean values for porosity and permeability are found from conventional $\boldsymbol{R}$ epresentative Volume Element (RVE) analysis. For sintered granular systems, the empirical constant in the classical Kozeny-Carman model is determined to be 131, while a value of 180 is expected for perfect mono-disperse sphere packings.

\section{$2.1 \quad$ Introduction}

Numerical and experimental investigations of fluid flow in porous and granular media are of crucial importance in many research areas, such as the recovery of hydrocarbons from oil reservoirs [35,81], ground water flow [17] or gas diffusion in fuel cells [82]. In spite of extensive scientific research, there are still many open questions namely which and how macroscopic transport factors other than porosity affect the fluid flow in a porous medium with a given microstructure $[53,60,82,118,175]$. An experimentally and numerically determinable parameter of porous materials is the intrinsic permeability which is highly sensitive to the underlying microstructure. The effective intrinsic permeability depends only on the pore structure of the medium, that is independent of the properties of the fluid. Therefore, a comparison with numerical determined permeability values based on Micro-X-Ray-Computer-Tomographic ( $\mu \mathrm{XRCT}$ ) images can increase the understanding of the effects of microstructure on the intrinsic permeability [75-77]. The typical workflow of $\mu \mathrm{XRCT}$-based permeability investigations comprises

1. the $\mu \mathrm{XRCT}$-scanning and reconstruction of the porous material; 
2. the filtering and segmentation of the image data;

3. the implementation of image data in numerical simulations;

4. validation of numerical calculations with experimental data;

5. finding new correlations between microstructure and macroscopic properties by combining the previous steps and insights.

The presented workflow clearly demonstrates that a numerical simulation based on real data sets and the correlation between microscopic and macroscopic properties can only be as good as the preceding filtering and segmentation procedure $[86,159]$. Therefore, a special focus lies on this topic. Possible ways are presented how to filter, segment and finally extract essential features of a porous material, which determine the intrinsic permeability.

In this work we investigate, both experimentally and numerically, the intrinsic permeability of artificial produced samples composed of sintered glass beads showing different particle diameters, porosities and degree of polydispersity. In contrast to common rock samples, like e.g. dolomite, sintered glass bead samples are characterized by their chemical stability and inertness, in addition to their relatively simple pore structure. Nevertheless, the pore structure, and thus the intrinsic hydraulical permeability can be influenced by the selection of certain glass beads and special sintering treatments compared to rock samples, like Fontainebleau sandstone. Another crucial advantage of sintered glass bead samples, in contrast to most rock samples, is the improved gray-scale contrast between the pore space and the solid phase, which considerably simplifies the image segmentation process and thus ensures a better comparability of experimentally and numerically determined permeability values. The filtering and segmentation procedure of sintered glass bead packings is further simplified by single phase composition of the solid matrix. In this respect, sintered glass bead samples can serve as replacement material for soil and rock specimen to provide a benchmark in permeability calculations.

The present paper focuses on the hydraulical properties of porous sintered glass bead systems. The influence of the sintering process on the microstructure, and thus on the permeability of the sintered samples are analyzed in detail by using appropriate XRCT data analysis and visualization methods (AVIZO Fire 8.0.1 and 9.0). In chapter 2 the well-known Darcy's law is introduced to define the intrinsic permeability of porous materials in general. Furthermore, the semi-empirical Kozeny-Carman equation, which is often used to determine the intrinsic permeability of granular systems, is presented and discussed in terms of microstructural parameters. In chapter 3 the Lattice Boltzmann (LB) method and the numerical set-up, which is used to determine the local intrinsic permeabilities of the extracted data sets, is described briefly. For a better understanding 
of the underlying microstructure, the sintering procedure is described in section 4.1. For validation of numerical data based on discretized $\mu \mathrm{XRCT} /$ voxel data sets of the produced sintered glass bead samples, the experimental setup and procedure of permeability measurements are described in section 4.2. The developed multi-purpose measuring cell is proposed in section 4.3. Chapter 5 focuses on the elaborate processing of $\mu \mathrm{XRCT}$ scans, whereby possible ways are introduced, how to filter, segment and extract essential features, which highly influences the hydraulical properties of porous sintered granular systems. Chapter 6 starts with presentations and discussion of results obtained from numerical and experimental porosity and permeability measurements. In addition, the results from RVE analysis for porosity and permeability and the peripheral porosity development of the samples are depicted and discussed in section 6.1. Moreover, the numerically determined local permeability values are qualitatively and quantitatively compared with the theoretical predictions according to the Kozeny-Carman model. Based on the Kozeny-Carman model further influence of parameters of hydraulical characteristics of sintered glass bead packings, like tortuosity, sphericity, specific surface area, particle sizes and pore throat distributions are visualized and analyzed by means of $\mu \mathrm{XRCT}$ data from section 6.2 to 6.4 . The study of hydraulical properties of porous sintered granular packings is concluded in section 2.7.

\subsection{Theory}

Darcy's law is the most commonly used empirical relationship for calculation of the pressure drop across a homogeneous, isotropic and non-deformable porous medium $[195,196]$. It states that, at the macroscopic level and in the creeping flow regime, the measured pressure drop $\Delta p / l$ per length applied to a porous medium, and the fluid flux per area $Q_{z} / A$ have a linear relationship given by

$$
\frac{Q_{z}}{A}=-\frac{k_{z}^{s}}{\mu^{f R}} \overbrace{\left(\frac{\Delta p}{l}-\rho^{f R} g\right)}^{=i},
$$

where $\mu^{f R}, A$ and $l$ are dynamic viscosity of the fluid, cross-sectional area and length of the sample [78]. The proportionality constant $k_{z}^{s}$ describes the intrinsic permeability of the porous medium in flow direction $z$, which strongly depends on the porosity and the microstructure (e.g particle shape, tortuosity and connectivity of pore channels). The term $\rho^{f R} g$ represents the gravity force-density, driving the fluid flow. The expression given in brackets is often referred to as the hydraulic gradient $i$.

The semi-empirical approach of Kozeny-Carman is one of the most well-known 
theories, which relates macroscopic parameters, like the intrinsic permeability, to microstructural parameters, like particle arrangement, shape and orientation or tortuosity (flow path) [51, 165, 196]. For granular media, the Kozeny-Carman permeability reads

$$
k_{z}^{s}=\frac{1}{2} \frac{\phi_{0}^{3}}{\left(1-\phi_{0}\right)^{2}}\left(\frac{1}{\alpha}\right)^{2}\left(\frac{1}{S_{v}}\right)^{2},
$$

where $S_{v}$ and $\alpha$ are the volume-based specific surface area and tortuosity of the fluid path. The porosity of the sample is denoted as $\phi_{0}$. For monodisperse granular media consisting of particles with diameter $d_{p}$ the intrinsic permeability is given by

$$
k_{z}^{s}=\frac{1}{180} \frac{\phi_{0}^{3}}{\left(1-\phi_{0}\right)^{2}} d_{p}^{2}
$$

Since in most investigated cases the granular medium consists of non-uniform spheres, Carrier et al. [41] have introduced an effective diameter $d_{r}$ which can be reliably determined on basis of the particle size distribution in accordance with

$$
d_{r}=\frac{100}{\sum f_{i} / \bar{d}_{i}},
$$

where $f_{i}$ is the fraction of particles between two sieve sizes and $\bar{d}_{i}$ corresponds to the geometrical average particle size between the minimum and maximum sieve size $\left(d_{\min , i}, d_{\max , i}\right)$

$$
\bar{d}_{i}=\sqrt{d_{\min , i} \cdot d_{\max , i}} .
$$

For monodisperse granular media the median diameter $d_{50}$ is commonly used as effective diameter due to the little variation in grain size. The median diameter is the value of the particle diameter at $50 \%$ in the cumulative distribution. In the case of a nearly symmetric particle size distribution, the median diameter is often replaced by the arithmetic mean value.

Several approaches have been introduced in recent years to describe the tortuosity (fluid path) in Equation (2.2) through porous media [51, 165, 175]. According to Berryman [21] for instance, the tortuosity can be estimated from the porosity by

$$
\alpha=1-r\left(1-1 / \phi_{0}\right),
$$

with $r=1 / 2$ as shape factor for spheres. A different approach for the determination of the geometrical tortuosity is from the centroids of two-dimensional slices 
obtained from $\mu \mathrm{XRCT}$ scans as

$$
\alpha=\frac{\sum_{i}^{N} d_{i}}{H}
$$

where $H=z(n)-z(0)$ represents the distance between two slices. The sum of all images $\sum_{i}^{N} d_{i}$ describes the total path length through the centroids of areas of each slice (cf. Figure 2.13, top left), and $d_{i}$ represents the distance between the centroids of area determined from two adjacent 2-dim slices.

\subsection{The lattice Boltzmann method}

For the determination of the numerical intrinsic permeabilities of the extracted differently sized subsets of glass beads the lattice Boltzmann method is used. We follow the procedure to measure permeabilities as described in Narvaez et al. [129, 130] and Frijters et al. [58, 59]. The LB method itself has proven to be very successful for modeling fluid flow in porous media [115]. It enables a straightforward implementation of complex boundary conditions and is suitable for use in parallel computation due to the high degree of locality of the algorithm. For the LB simulations the Boltzmann equation

$$
\frac{\partial}{\partial t} f(\mathbf{x}, \mathbf{c}, t)+\mathbf{c} \cdot \nabla f(\mathbf{x}, \mathbf{c}, t)=\Omega(f(\mathbf{x}, \mathbf{c}, t))
$$

in discretized form is solved to simulate creeping fluid flow through porous media. Equation (2.8) describes the evolution of a single-particle probability density $f(\mathbf{x}, \mathbf{c}, t)$, whereby $\mathbf{x} \in \mathbb{R}^{3}$ is the position vector, $\mathbf{c} \in \mathbb{R}^{3}$ is the velocity vector, $\mathrm{t} \in \mathbb{R}$ is the time and $\Omega(f(\mathbf{x}, \mathbf{c}, t))$ is the collision operator describing binary collisions between particles. The time discretization is performed by using a time step $\Delta t$, whereas for the lattice velocities a finite set of vectors $\mathbf{c}_{i}$ with $i=1 \ldots 19$ lattice points is applied. The position vector $\mathbf{x}$ is discretized by using a three-dimensional structured cubic lattice with a lattice constant $\Delta x$. For the permeability calculations the simulation parameters are chosen in accordance to Narvaez et al. [129] and Frijters et al. [58, 59]. As in those publications the well-known D3Q19 lattice for velocities, providing adequate accuracy at moderate computational cost is applied [148]. For the collision matrix $\Omega$ in Equation (2.8) a standard two relaxation time (TRT) model is used, c.f. Ref. [49, 129]. Narvaez et al. [129, 130] have shown that relaxation times of $\tau=1$ and $\tau_{b u l k}=0.84$ provide useful results for permeability calculations.

The porous sample is positioned between two fluid chambers which serve as inand output to avoid artifacts, cf. Figure 2.4 (left). The on-site boundary condition introduced by Zou and He [200] and later extended to three dimensions and 
nineteen velocities (D3Q19) by Hecht et al. [79] is applied to generate a gradient in flow direction ( $z$-direction) by defining the fluid densities at the in- and outlet of the system,

$$
\begin{aligned}
\rho^{f R}(z=1) & =1+\Delta \rho^{f R}, \\
\rho^{f R}\left(z=n_{z}\right) & =1-\Delta \rho^{f R},
\end{aligned}
$$

whereby $n_{z}$ indicates the total number of lattice nodes in flow direction and $\Delta \rho^{f R}$ the difference of the fluid density between in- and outlet. To ensure creeping flow with low Reynolds numbers the density difference is chosen in the order of $10^{-4}$ in lattice units. Furthermore, the computational domain is surrounded by walls in $x$ - and $y$-directions to prevent flow over boundaries orthogonal to the pressure gradient. The pressure gradient in flow direction $z$ reads then as

$$
(\nabla p)_{z}=\frac{p\left(z=n_{z}\right)-p(z=1)}{n_{z}}=\frac{2 \Delta \rho^{f R}}{3 n_{z}} .
$$

The intrinsic permeability $k_{z}^{s}$ in flow direction $z$ can be expressed in terms of the lattice Boltzmann relaxation time as

$$
k_{z}^{s}=\frac{2 \tau-1}{6} \frac{3 Q_{z} n_{z}}{2 \Delta \rho^{f R}\left(n_{z} n_{y}\right)},
$$

whereby $A=n_{z} n_{y}$ represents the cross-sectional area of the extracted subset, $n_{z}$ the sample length and $Q_{z}$ the volume flux in $z$-direction. The system has reached steady state when the mass flux and the permeability are constant over the geometry [58, 129]. For the determination of the permeability of the differently sized samples in flow direction $z$ the permeabilities are averaged over the lattice surfaces in flow direction $z$.

\subsection{Experiments}

\subsubsection{Sintering}

In this study various types of glass with different chemical compositions and characteristic particle diameters are used for sintering, see Table 2.1. Depending on the composition, the deformation temperature of the used glass particles varied between 575 and $680{ }^{\circ} \mathrm{C}$. The used glass beads (Muehlmeier $\mathrm{GmbH} \& \mathrm{Co} . \mathrm{KG}$ ) showed different particle sizes and degree of polydispersity, cf. Table 2.1. The specific density of the sintered glass beads lies at $2.5 \mathrm{~g} / \mathrm{cm}^{3}$.

It is attempted to generate cylindrical samples and at the same time to ensure the smallest possible deformation of the beads during the sintering process, see 
also Ref. [71]. Since the used glass beads showed an almost ideal spherical shape before sintering, the non-sphericity determined from the $\mu \mathrm{XRCT}$ analysis can be used to quantify the deformation degree of the beads as result of sintering. The sphericity describes how spherical an object is and is defined as the ratio of the surface area of a sphere with the same volume as the given particle to the surface area of the particle itself:

$$
\psi_{s}=\frac{\pi^{\frac{1}{3}}\left(6 V_{p}\right)^{\frac{2}{3}}}{A_{p}},
$$

where $V_{p}$ and $A_{p}$ describe the (voxel-based) volume and surface area of the given particle. The sphericity of an ideal sphere is 1 , whereas for non-spherical particles, the sphericity is $\psi_{s}<1$, such that $1-\psi_{s}$ quantifies the non-sphericity.

The produced cylindrical samples have bulk diameters of 30 and $50 \mathrm{~mm}$, and the lengths of the specimens are $50 \mathrm{~mm}$. Figure 2.1 (left) shows the experimental sintering set-up for the glass beads. The sintering of the glass beads is performed in a tubular furnace with heat power of $0.7 \mathrm{~kW}$ and a nominal target temperature of $1000^{\circ} \mathrm{C}$ under atmospheric conditions. The induction furnace is equipped with three programmable temperature controllers (type West 5010) regulating the inner temperature at three different furnace zones. The temperature progression within the furnace is monitored continually at five different places during the sintering process using thermocouples (type K), see Figure 2.1 (left). The measured temperature curves are depicted in Figure 2.1 (right). The glass beads are filled in a quartz glass cylinder (with inner diameters of approximately 30 and $50 \mathrm{~mm}$ ) and completely enclosed with graphite paper. The melting temperature of the quartz glass cylinder is around $1713{ }^{\circ} \mathrm{C}$ and thus certainly higher than the deformation temperature of the glass beads $\left(\approx 690^{\circ} \mathrm{C}\right)$. The graphite paper prevents an adhesion or sticking between the beads and the cylinder. The glass beads are manually shaken prior to sintering to reach the closest glass bead packing. The samples are subsequently loaded from the top with different masses ranging between 100 and $300 \mathrm{~g}$ corresponding to pressures of $1.39 \mathrm{kN} / \mathrm{m}^{2}$ and $4.16 \mathrm{kN} / \mathrm{m}^{2}$ for samples with $30 \mathrm{~mm}$ diameter or $0.50 \mathrm{kN} / \mathrm{m}^{2}$ and $1.50 \mathrm{kN} / \mathrm{m}^{2}$ for samples with $50 \mathrm{~mm}$ diameter, respectively. As can be seen in Figure 2.1 (right) the glass beads are heated up with a constant temperature rate of $300^{\circ} \mathrm{C}$ per hour until the required sintering temperature of $695^{\circ} \mathrm{C}$ is reached. Holding the beads at this temperature for approximately 2.5 hours, the specimen is finally cooled down in an uncontrolled manner by switching off the furnace, cf. temperature curve at position 3 in Figure 2.1 (right). Since the prepared samples are placed centrally in the furnace, only the temperature curve at position 3 is of major relevance. The preprogrammed target temperature of $695^{\circ} \mathrm{C}$ in the outer zones (position 1 and 5) is not reached due to large heat losses at the top and bottom of the furnace whereas the target temperature at positions 2 and 4 is reached, but delayed by approximately 1-2 hours. 
Table 2.1: Material parameters and characteristic particle diameters of the investigated glass beads.

\begin{tabular}{|c|c|c|c|c|c|c|}
\hline $\begin{array}{l}\text { Material de- } \\
\text { scription }\end{array}$ & $\begin{array}{c}\text { Diameter }^{[1]} \\
{[\mathrm{mm}]}\end{array}$ & $\begin{array}{l}\mathrm{D}_{10}{ }^{[2]} \\
{[\mu \mathrm{m}]}\end{array}$ & $\begin{array}{l}\mathrm{D}_{50}{ }^{[2]} \\
{[\mu \mathrm{m}]}\end{array}$ & $\begin{array}{l}\mathrm{D}_{90}{ }^{[2]} \\
{[\mu \mathrm{m}]}\end{array}$ & $\begin{array}{c}\mathrm{D}[4,3]^{[2]} \\
\quad[\mu \mathrm{m}]\end{array}$ & $\begin{array}{c}\mathrm{D}[3,2]^{[2]} \\
{[\mu \mathrm{m}]}\end{array}$ \\
\hline \multirow[t]{2}{*}{ Minibeads ${ }^{[A]}$} & 0.4-0.6 & 388 & 519 & 696 & 532 & 505 \\
\hline & $0.6-0.8$ & 507 & 683 & 916 & 702 & 666 \\
\hline \multirow[t]{2}{*}{ Minibeads $^{[B]}$} & $0.8-1.0$ & 646 & 883 & 1222 & 915 & 862 \\
\hline & $1.0-1.2$ & 766 & 1045 & 1415 & 1073 & 1016 \\
\hline \multirow[t]{2}{*}{ Glass Beads } & $1.5-2.0$ & 857 & 1190 & 1581 & 1209 & 1143 \\
\hline & $2.0-2.5$ & - & - & - & - & - \\
\hline $\begin{array}{l}\text { "Diamond" } \\
\text { Pearls }[D]\end{array}$ & $3.0( \pm 0.2)$ & - & - & - & - & - \\
\hline
\end{tabular}

Chemical Composition [wt\%]:

$[A]: 70-74 \mathrm{SiO}_{2}, 10-15 \mathrm{Na}_{2} \mathrm{O}, 7-11 \mathrm{CaO}, 3-5 \mathrm{MgO}, 0.5-2 \mathrm{Al}_{2} \mathrm{O}_{3}$

${ }^{[B]}$ : $60-70 \mathrm{SiO}_{2}, 12-18 \mathrm{Na}_{2} \mathrm{O}, 15-20 \mathrm{CaO}, 1-4 \mathrm{MgO}, 1-5 \mathrm{Al}_{2} \mathrm{O}_{3}, 1.4 \mathrm{MgO},<0.1$ $\mathrm{Fe}_{2} \mathrm{O}_{3}$

${ }^{[C]}$ : $72.3 \mathrm{SiO}_{2}, 14.3 \mathrm{Na}_{2} \mathrm{O}, 7.5 \mathrm{CaO}, 1.4 \mathrm{MgO}, 2.4 \mathrm{Al}_{2} \mathrm{O}_{3}, 1.4 \mathrm{MgO},<0.1 \mathrm{Fe}_{2} \mathrm{O}_{3}$

$[D]: 61-67 \mathrm{SiO}_{2}, 10-18 \mathrm{Na}_{2} \mathrm{O}, 5-10 \mathrm{CaO}, 0.5-3 \mathrm{MgO}, 3-8 \mathrm{Al}_{2} \mathrm{O}_{3}, 0.5-3 \mathrm{MgO}, 1-5$

$\mathrm{B}_{2} \mathrm{O}_{3}$

[1] manufacturer information, [2] obtained from laser granulometry measurement (for description of the characteristic diameters see Ref. $[9,10]$ )

Depending on the chemical composition, bead diameter, dead loads of the used masses and sintering duration, the initial heights of the untreated specimens shrink by 1 to $5 \mathrm{~mm}$. After sintering the samples are cut by a diamond disc to the desired length of $50 \mathrm{~mm}$.

\subsubsection{Permeability measurement set-up}

Technical requirements on precise hydraulical measurements are very high [29, $50,52]$. Therefore, an elaborate setup is built for the stationary permeability measurements, to guarantee proper comparability between numerical and experimental permeabilities, see Figure 2.2 (left). To minimize the content of air bubbles and to guarantee for reproducible experimental results, use is made of filtered and de-aired water. For this purpose, the water is mechanically filtered in various filter stages until reaching a degassing tank, where the filtered water 

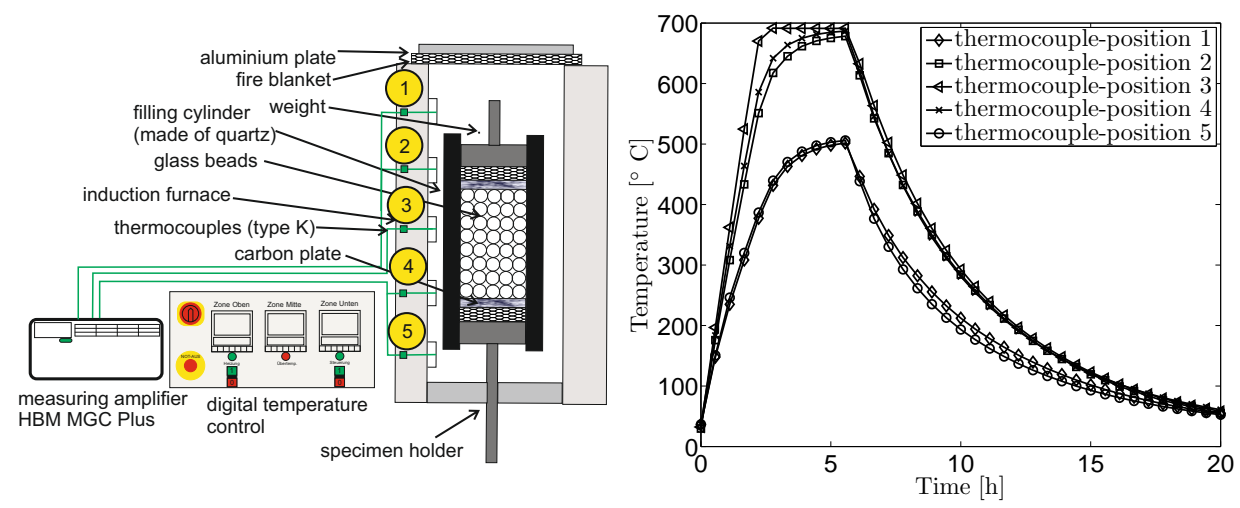

Figure 2.1: Experimental arrangement for sintering (left) and measured temperature curves at five different furnace zones (right).

is de-aired.

The measuring cell including the hose connections are rinsed with carbon dioxide before the cell is flooded with filtered and de-aired water. The carbon dioxide easily dissolves in water. In this way, the content of air bubbles in the cell is minimized and an optimal water saturation of the sample is achieved.

The stationary permeability experiments are performed by controlling the volumetrical flux through the pressure regulator of the degassing tank. During the permeability measurements, the volume flux is stepwise increased by increasing the inertial pressure of the degassing tank through a pressure regulator, cf. and Figure 2.3 (left). The pressure regulator ensures that the atmospheric pressure in the degassing tank remains constant. In this manner, different measuring ranges for fluid flux and pressure difference are driven to determine the intrinsic permeability of the produced sintered specimens, see Figure 2.3. The measured values for pressure difference and fluid flux are continuously recorded (digital data acquisition, sampling rate $0.5 \mathrm{~Hz}$ ) during each measurement and subsequently sent to a computer. Depending on the porosity and glass bead diameter of the sintered specimens, different fluid fluxes ranging between 30 and $245 \mathrm{ml} / \mathrm{min}$. are observed. With these volume flows pressure differences up to $35 \mathrm{mbar}$ can be generated. For each measurement the water temperature is measured and found to be in the narrow range of $20^{\circ} \mathrm{C}<T<22^{\circ}$. 

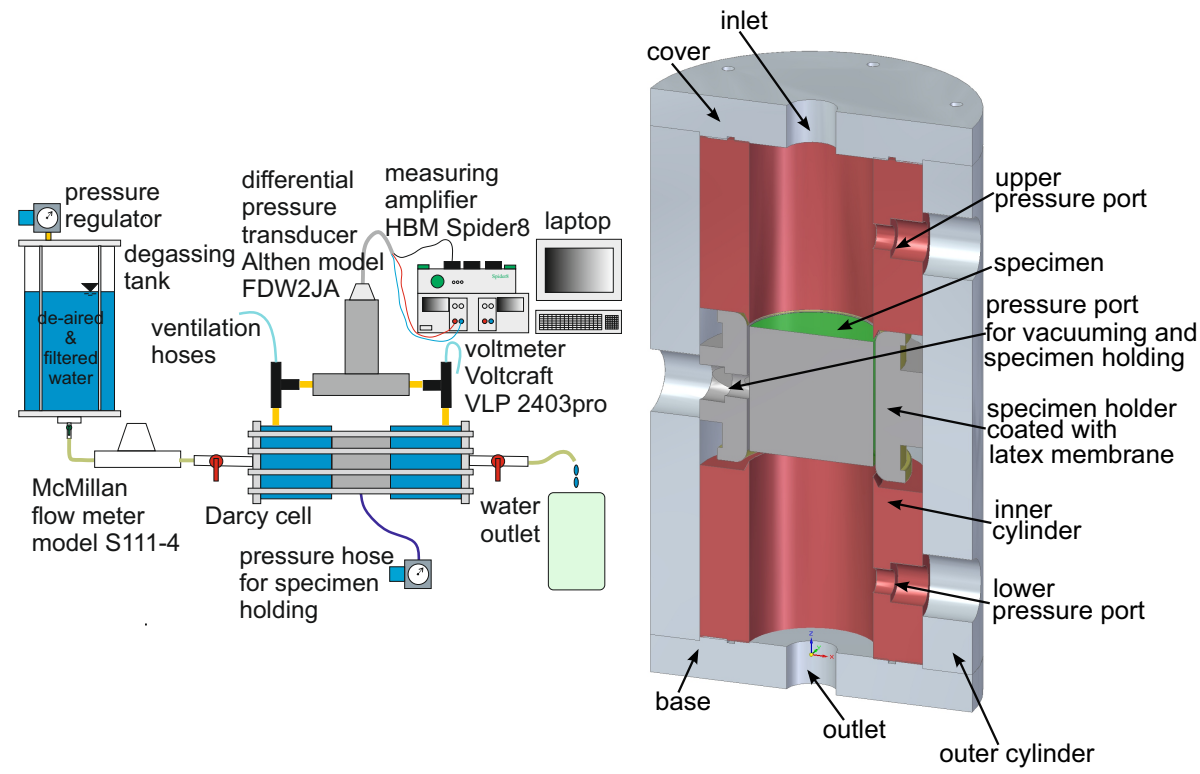

Figure 2.2: Experimental set up for stationary permeability experiments (left) and detailed construction drawing of the multi-task measuring cell in mode for permeability measurements (right).
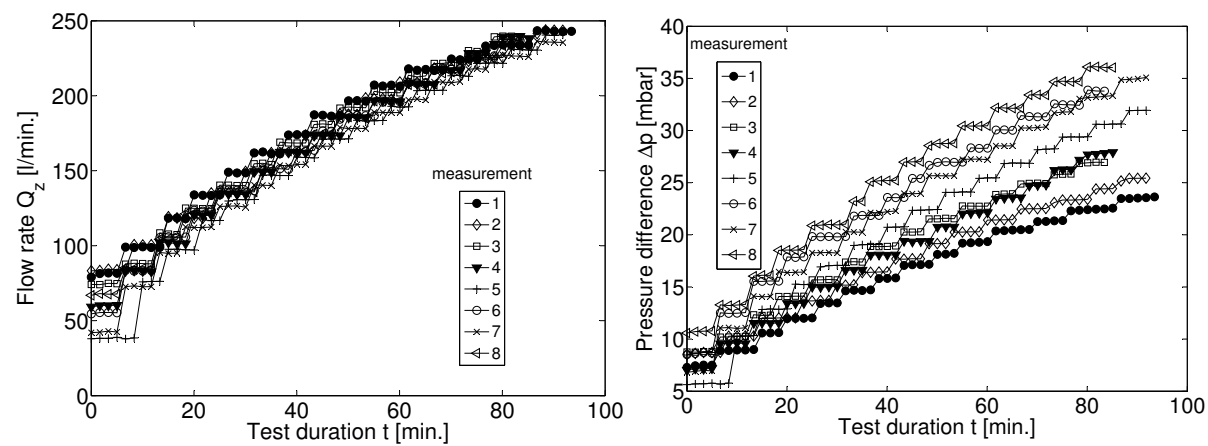

Figure 2.3: Stepwise increase of flow rate $Q_{z}$ and differential pressure $\Delta p$ caused by viscous fluid flow through the sintered glass beads sample in dependence of measuring time $t$. 


\subsubsection{Measuring Cell}

The developed measuring cell has been designed according to a modular concept in order to use it in various applications. Figure 2.2 (right) shows an illustration of the measuring cell in operating mode for stationary permeability measurements.

It consists of an inner and outer cylinder. The inner cylinder is made of an acrylic glass (PMMA) and produced in various sizes, whereas the outer cylinder is made of aluminium (AlCu4PbMgMn alloy) to stabilize the measuring cell. As can be seen from Figure 2.2 (right), the sintered sample is positioned at the center of the cell and pneumatically fixed by a specially developed specimen holder coated with a $1 \mathrm{~mm}$-thick latex membrane. During flow measurements a static air pressure on the latex membrane is applied which fixes the specimen in the current position and prevents a surrounding fluid flow. At the same time, the latex membrane ensures a hermetically sealing off the measuring cell to the outside. The pressure difference, mainly resulting from the viscous friction of the fluid passing through the porous sintered sample, can be taken via the upper and lower pressure port and measured by a high-precision differential pressure transducer (type FDW2JA, ALTHEN, Germany) capturing differential pressures up to 35 mbar (with an accuracy of $0.25 \%$ ).

\section{$2.5 \quad \mu \mathrm{XRCT}$ data processing}

$\mu \mathrm{XRCT}$ data processing is a crucial step towards understanding of fluid flow through complex morphologies like sintered glass beads. It is a important tool for visualization and quantification of parameters, such as porosity, tortuosity or pore throats, determining the hydraulical conductivity of a porous medium $[145,190,191]$. The correct procedure of the $\mu$ XRCT-data including an adequate filtering and thresholding method is essential for a proper comparison between experimental and numerical determined permeabilities [86, 197].

The XRCT device used for imaging of the sintered samples is a 'nanotom 180' device provided by the petrophysics laboratory at the Leibniz Institute for Applied Geophysics in Hannover, Germany. The device is equipped with a special watercooled nanofocus X-ray tube with a maximum $180 \mathrm{kV}$ and $15 \mathrm{~W}$. The minimal focus size is about $0.6 \mu \mathrm{m}$, which results in a detail detectability of $0.2 \mu \mathrm{m}$ [75]. The measurement parameters for the investigated scans are $107 \mathrm{kV}$ and $200 \mu \mathrm{A}$. XRCT images at 900 angles on $360^{\circ}$ are performed with an integration time of $10 \times 0.1$ seconds per angle. The initial voxel resolution of the XRCT scans is 16 $\mu \mathrm{m}$ and remained constant during the whole $\mu \mathrm{XRCT}$ data processing.

Figure 2.4 (right) shows the main image processing steps. Starting from raw data, 


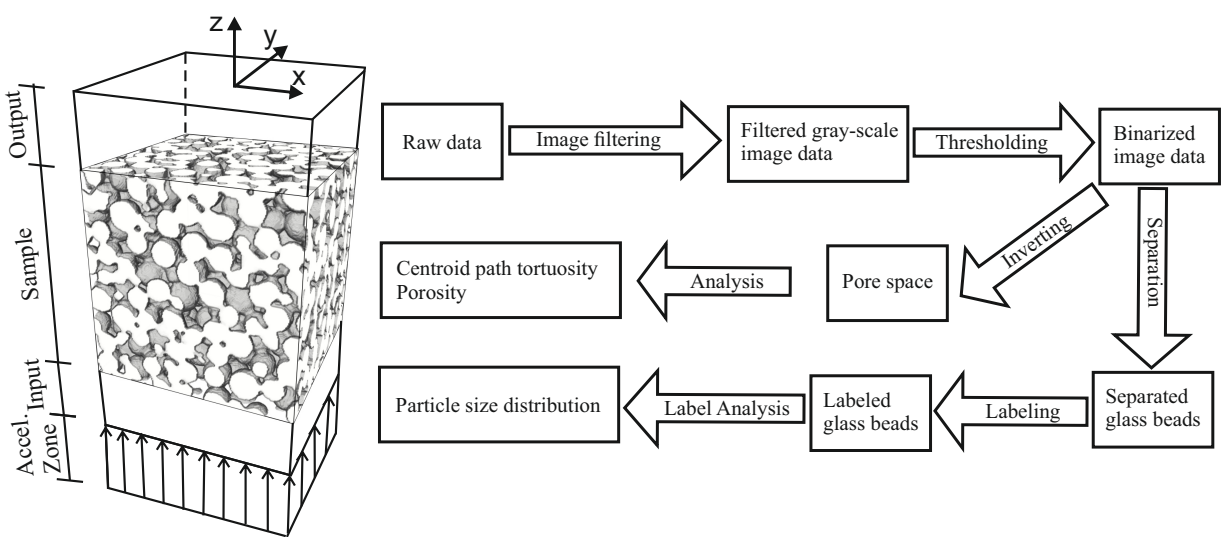

Figure 2.4: (Left) Computational domain in LB simulations consisting of a porous glass bead sample and the two fluid chambers denoted as in-and output. The fluid is accelerated upwards in the acceleration zone at bottom. (Right) Process flow chart used for extracting influence parameters and quantities determining the hydraulical properties of the sintered glass bead samples from CT-data.

the image file is filtered in several stages until the desired gray scale distribution is reached. For better visualization of the filter effect, Figure 2.5 demonstrates exemplarily the filtering procedure on the basis of slices applied onto the original raw data with the corresponding gray-scale value distribution. For the sake of clarity, during filtering the image is interpreted and processed as a 3-dim volume , and the gray-values of each voxel are numbers of decimals obtained from 16-bit binary representation of the gray level. In the initial state (state A), the grayscale distribution of the raw data show two peaks which can be clearly attributed to the pore space and the glass beads. Starting from the original raw data a simple logical operator with the so-called "arithmetic" module is applied to remove bright spots from the image file. These bright spots highlighted by a red circle in state A are caused by density fluctuations and chemical impurities of the beads and can be clearly assigned to greater gray-scale values. In the first filtering step, the gray values belonging to these bright spots are lowered artificially by setting a defined maximum threshold for gray-scale values. As highlighted in the example, a maximum threshold of 26818 is used, cf. Figure $2.5 \mathrm{~B}$ ).

Since the gray-scale values belonging to the glass beads and pore space overlap due to their wide distributions, the segmentation procedure becomes difficult. Therefore, in the second step of the filtering procedure the so-called "delineate" filter is applied to enhance the edges of the glass beads and to adjust the contrast between the pore space and the glass beads. Local changes in intensity of gray- 
scale values constitute a common issue during segmentation. The "delineate" filter, which is based on a phase contrast method, can detect sharp transitions between different phases to finally enhance and contrast the edge of an object to be segmented. As a result, the gray-scale distributions between the glass beads and the pore space are clearly separated from each other (state C), which simplifies the further separation of the glass beads from the pore space.

In the last step, the "median" filter is applied to denoise and smooth the image data. This filter uses morphological operators to set the gray-scale value of a voxel to the median for a defined neighborhood [157]. The gray-scale value distribution from state $\mathrm{C}$ is changed only slightly compared to the distribution in state D. Because of the high data quality, which comprises also the high gray-scale contrast between pore space and solid matrix, there is no need to use the so-called "nonlocal means" filter, which is commonly used to denoise image data especially at the edge of an object to be segmented $[32,33,86]$. The algorithm of this filter compares the neighborhood of a voxel in a given search window with neighbors of the current voxel. A weight is determined from the similarity between the neighbors, with which the values of the voxel value in the search window will influence the new value of the current voxel. The final weight result by applying a Gaussian kernel to the similarity values $[32,33]$. In accordance with the process flow chart shown in Figure 2.4 (right), the filtered gray-scale image data is thresholded to generate a binary map. The threshold is selected manually for each sample in a way that the solid glass beads are assigned to values of unity and the pore space voxels are set to zero. For the determination of porosity and visualization of the pore space the binarized image data is inverted. In the following step, the module "axis connectivity" is used to create a binary image containing all path linking planes. Subsequently, the so-called centroid path tortuosity module is applied to the inverted and binarized image data to determine the geometrical tortuosity, cf. Figure 2.13. This module allows the computation of the tortuosity of a path according to Equation (2.7) which is formed by the centroids of each plane.

\section{Particle size distribution}

For the determination of the particle size distribution, the segmented glass beads are separated using a high-level combination of watershed, distance transform and numerical reconstruction algorithms. After separation, the segmented beads are numerated with the "labeling" module. In this module, each voxel of the same object is assigned to the same value, and each object gets a different value. Based on the labeled system a quantitative analysis is performed to determine specific parameters, like surface area, volume or volume-equivalent diameter of 


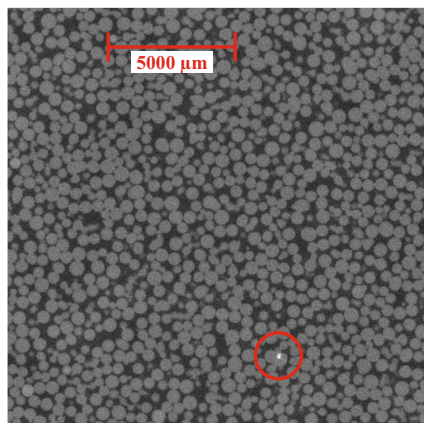

A.)
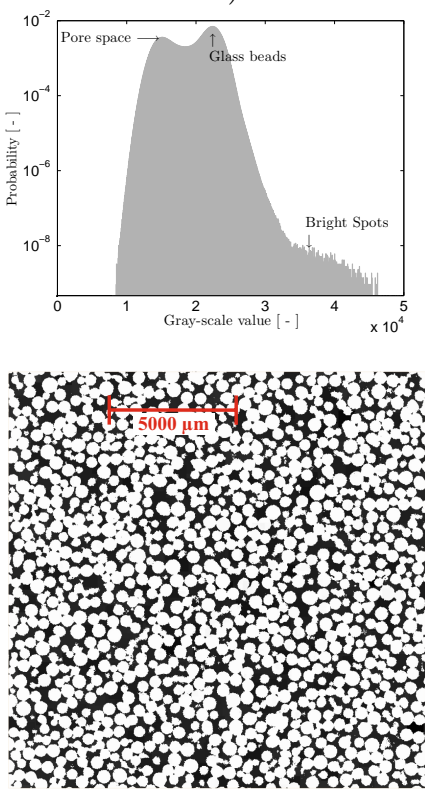

C.)

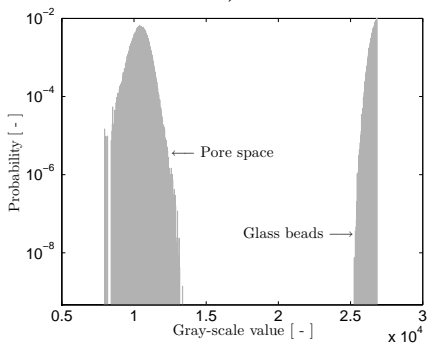

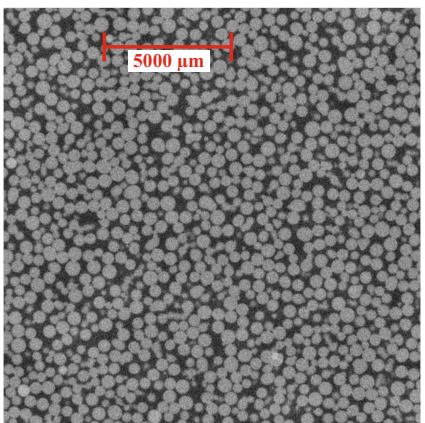

B.)

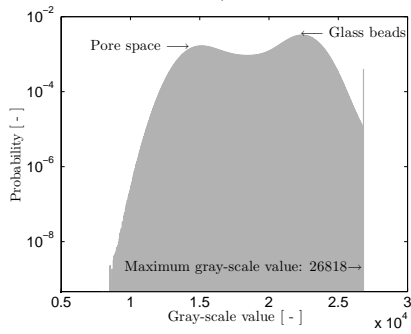

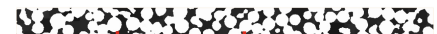

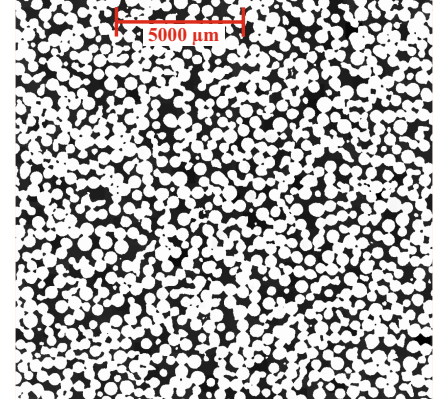

D.)

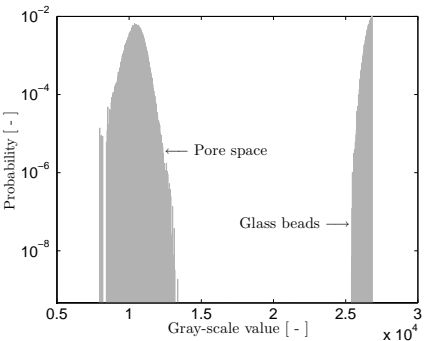

Figure 2.5: Illustration of the applied multistage filtering process using slice images with 1024 x 1024 pixels: A.) Untreated raw $\mu \mathrm{CT}$ scan data B.) after removing bright spots, C.) after applying the "delineate" filter D.) after applying the "median" filter. The corresponding gray value distributions of the images after each filter step are shown below in the respective panels. The vertical axis is logarithmic and gives the probability of occurrence of the gray values, while the horizontal axis represents the gray value of a voxel. 
Table 2.2: Comparison of characteristic parameters from particle number distributions gained from $\mu \mathrm{m}-\mathrm{CT}$ and laser granulometry.

\begin{tabular}{|c|c|c|c|c|c|c|}
\hline & Unit & $\begin{array}{l}256 \text { voxel } \\
\text { cube }\end{array}$ & $\begin{array}{l}512 \text { voxel } \\
\text { cube }\end{array}$ & $\begin{array}{l}1024 \text { voxel } \\
\text { cube }\end{array}$ & $\begin{array}{l}\text { Initial } \\
\text { cuboid }\end{array}$ & $\begin{array}{l}\text { Laser } \\
\text { granulom- } \\
\text { etry }\end{array}$ \\
\hline $\begin{array}{l}\text { Number of } \\
\text { particles } N\end{array}$ & {$[-]$} & 705 & 5,144 & 39,290 & 78,375 & - \\
\hline $\begin{array}{l}\text { Arithmetic } \\
\text { mean value } \\
\left\langle d_{p}\right\rangle\end{array}$ & {$[\mu \mathrm{m}]$} & 383.33 & 499.59 & 499.59 & 499.59 & 464.88 \\
\hline $\begin{array}{l}\text { Standard } \\
\text { deviation } \\
\sigma_{d_{p}}\end{array}$ & {$[\mu \mathrm{m}]$} & 216.74 & 286.06 & 286.06 & 286.06 & 326.78 \\
\hline
\end{tabular}

each particle object. The particle diameter $d_{p}$ is computed by

$$
d_{p}=\sqrt[3]{\frac{6 V_{p}}{\pi}}
$$

where $V_{p}$ is the voxel-based volume of the particle. As a result, Figure 2.6 shows (from state A to $\mathrm{C}$ ) the volume rendered sample in the form of a cuboid with the dimensions $1024 \times 1024 \times 2048$ voxel $^{3}$ in the raw, and the segmented and labeled state. In the labeled state different colors are used to distinguish optically between the identified glass particles. It should be stated, that the coloring of the particles occurs randomly. After a certain number of beads the coloring is repeated and the same colors are assigned again to different identified objects. The initial cuboid shown in Figure 2.6 contains more than 78,000 particles with diameters ranging mostly between 0.4 and $0.6 \mathrm{~mm}$. The corresponding particle number distributions of the initial cuboid and different sized subsets taken from the initial cuboid are depicted in Figure 2.6 (D). For all investigated subsets a monomodal distribution with maximum around $500 \mu \mathrm{m}$ occurs, which confirms the representativeness of the investigated subsets, except for the smallest one. For comparison, the particle number distribution obtained from laser granulometry measurement (Mastersizer 2000, Malvern Instruments Ltd.) before sintering the glass beads shows a broader distribution of the particle diameters compared to the distributions gained from the $\mu \mathrm{XRCT}$ data. The characteristic particle diameters obtained by laser granulometry measurements of all investigated samples are summarized in Table 2.1, and the arithmetic mean values and the standard deviations of the particle number distributions shown in Figure 2.6 (D) are given in Table 2.2. 


\section{Pore throat determination}

A decisive factor, which determines the intrinsic permeability in granular porous systems is the pore throat area. Micro tomographic imaging techniques enable to localize, visualize and quantify such determining areas.

Therefore, Figure 2.7 (top) illustrates exemplarily the main image processing steps for determination of pore throats using a 256 voxel-sided cube showing glass bead diameters between 2.0 and $2.5 \mathrm{~mm}$. Starting from the filtered grayscale image the glass beads are binarized by using the tool of interactive thresholding. Hereby each voxel is assigned to a value of one (solid) or zero (void) by defining a threshold grayscale value as described in the previous section 2.5. In the following step the segmented glass beads are inverted to determine the pore space of the cube. After inversion the segmented pore space are separated in various pore voids by using the tool of "binseparate". This module computes the watershed lines of a binary image. In the separation process voxels with at least one common edge are considered as connected and the separation takes place at the narrowest places of the pore space. In the next step, the separated pore space is deducted from the untreated pore space, in order to determine the split planes, which also represent the pore throat areas. After labeling typical pore throat areas formed by three or four particles result, see Figure 2.7 (bottom). From the computed pore throat areas an equivalent pore throat diameter

$$
d_{p t}=\sqrt{\frac{4 A_{p t}}{\pi}}
$$

is determined, whereby $A_{p t}$ represents the pore throat area.

\subsection{Results and discussion}

In this section the results of the determining parameters of the intrinsic permeability, described in previous sections, obtained from $\mu \mathrm{XRCT}$ analysis and LB simulations are presented successively, discussed and compared with experimental results. Moreover, the dependency of the intrinsic permeability on different parameters and on the localizations and sizes of the investigated subsets are analyzed qualitatively and quantitatively. 


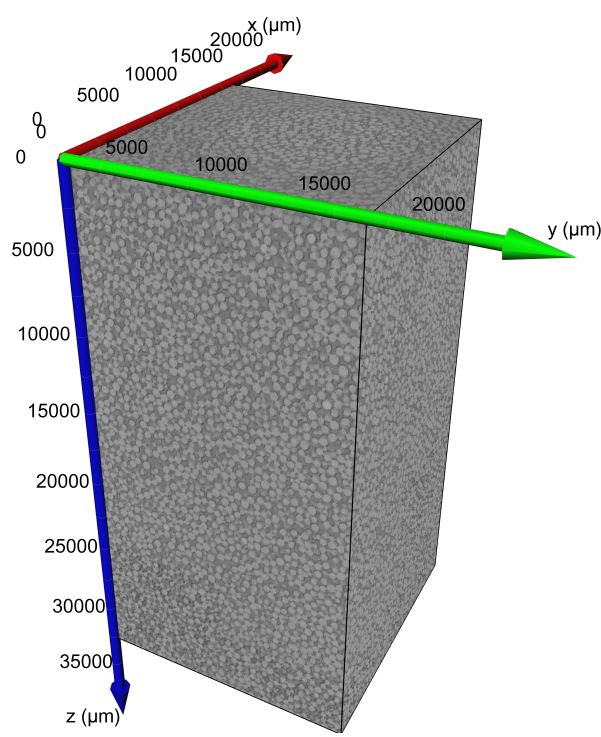

A.)

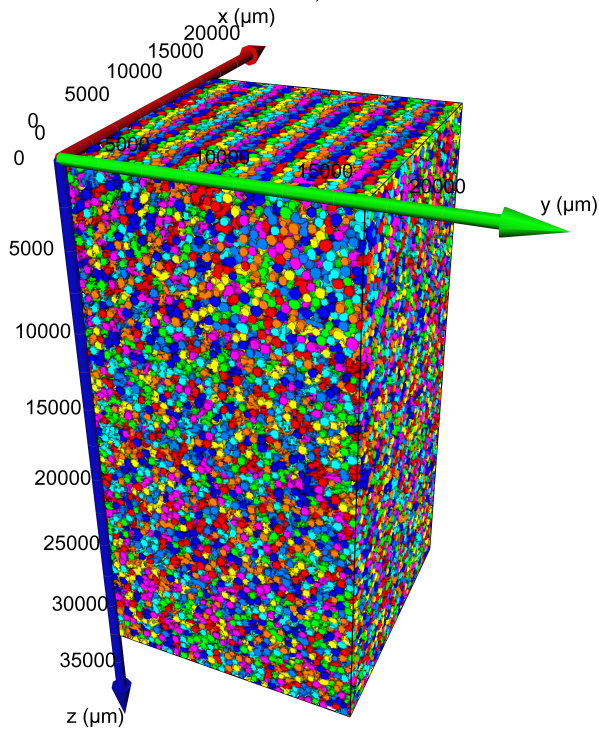

C.)

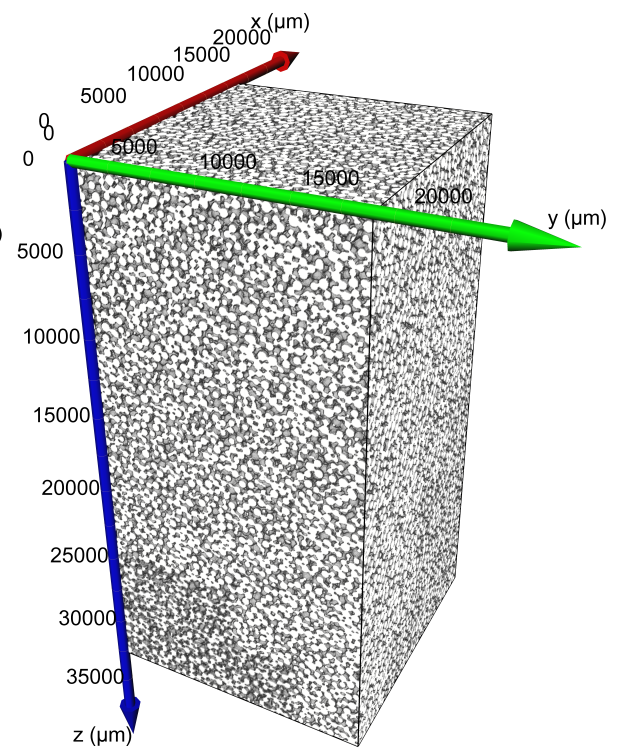

B.)

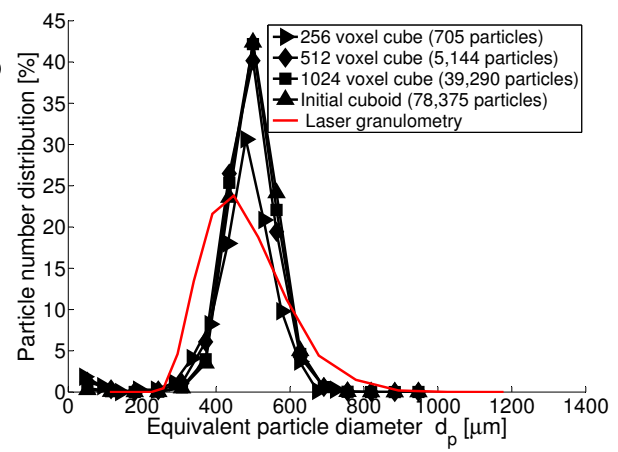

D.)

Figure 2.6: Volume rendering of the 3D initial cuboid with the dimensions $1024 \times 1024 \times 2048$ voxel $^{3}$, whereby the voxel resolution is $16 \mu \mathrm{m}$. The illustration on top-left shows the initial cuboid before segmentation in the raw state A. State B represent the binarized 3D image after segmentation and state C shows the separated and labeled system used for determination of the particle number distribution. In panel D., the resulting particle number distributions of differently sized subsets taken from different positions of the entire scanned region in comparison with measurement data obtained from laser granulometry are presented. The initial cuboid contains 78,375 particles, while the other distributions are based on less particles as given in the inset. 

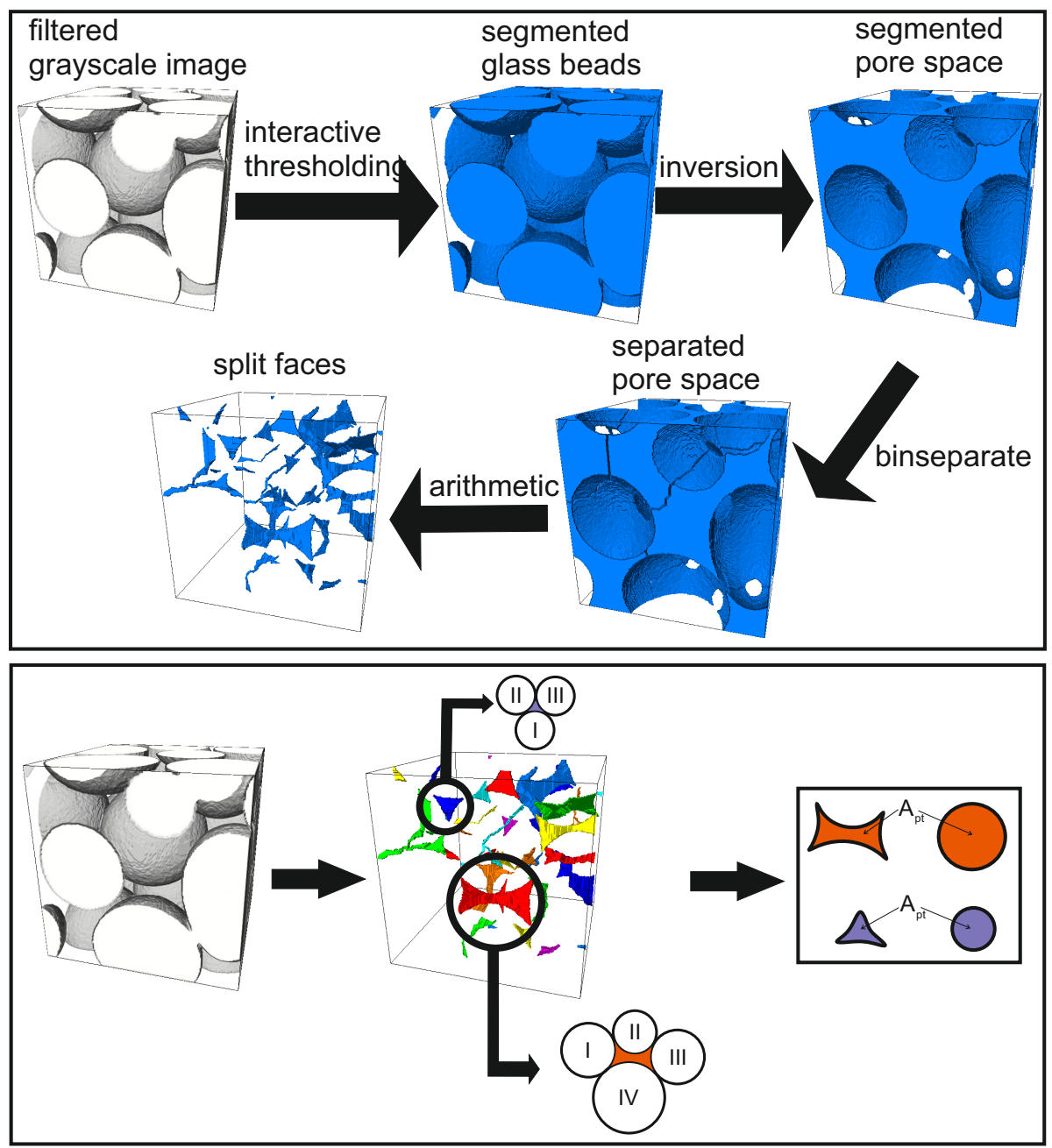

Figure 2.7: (Top) Illustration of main image processing steps for visualization and quantification of pore throat areas in AVIZO 8.0.1. (Bottom) Resulting pore throat areas in a slightly polydisperse sintered glass bead packing and the principle for determining equivalent pore throat diameter is shown below. 

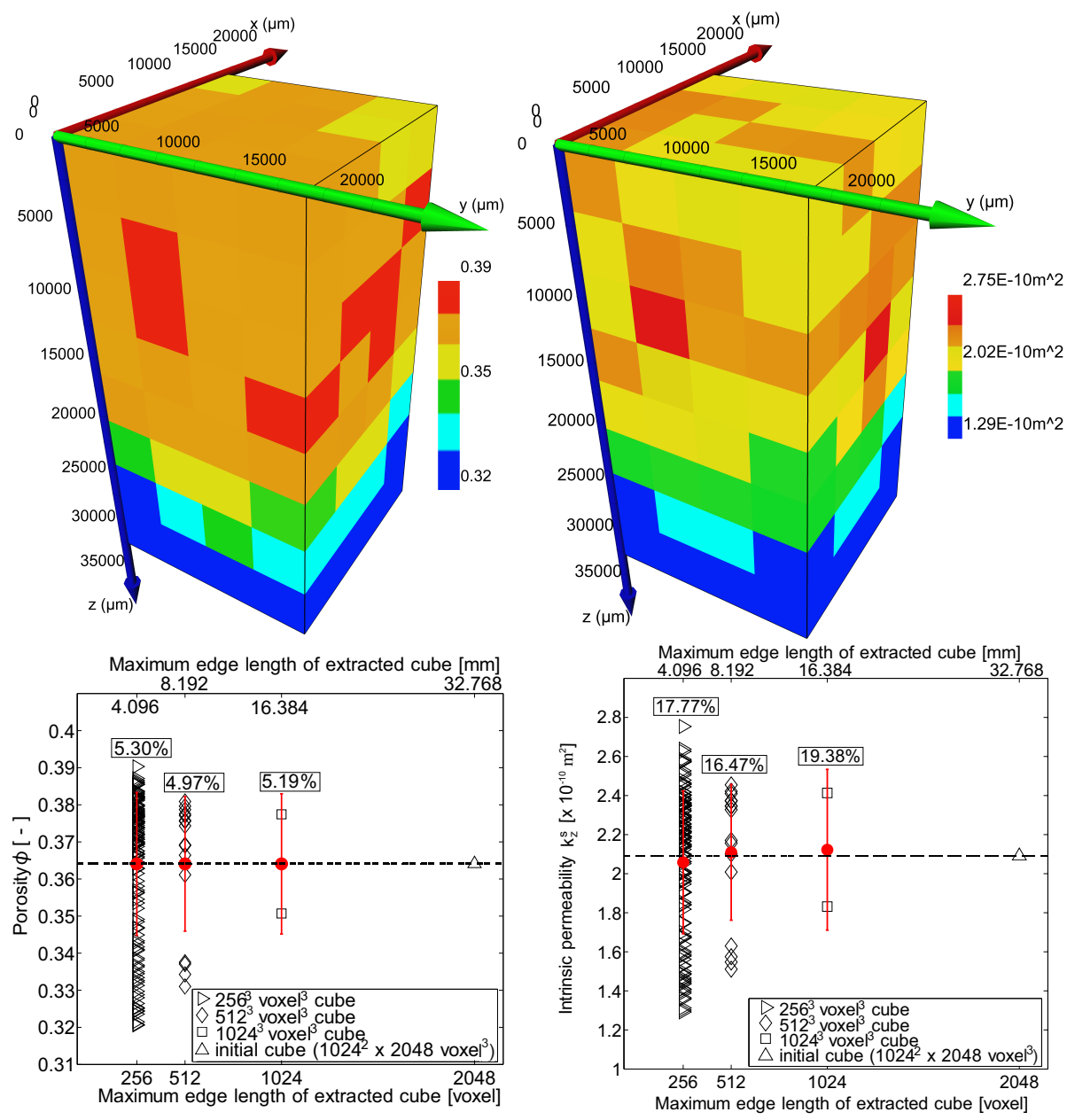

Figure 2.8: Local distribution of numerically determined porosity and permeability values of subsets with edge lengths of 256 voxels within the initial cuboid with dimensions of $1024 \times 1024 \times 2048$ voxel $^{3}$. The conventional representative volume element (RVE) analysis for porosity and permeability are shown below. 

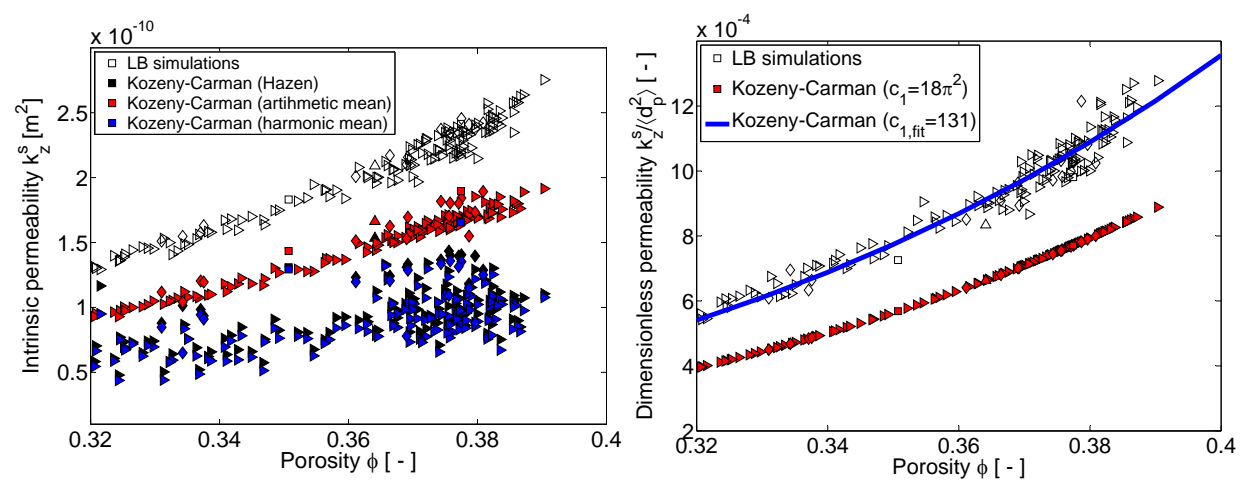

Figure 2.9: (Left) Local intrinsic permeabilities of different subsets showing particle diameter between 0.4 and $0.6 \mathrm{~mm}$ as a function of porosity. (Right) Normalized permeabilities of differently sized subsets (with maximum edge lengths of $\triangleright$ - 256 voxel, $\diamond-512$ voxel, $\square-1024$ voxel, $\triangle$ - 2048 voxel) from LB simulations and predicted values according to Kozeny-Carman in dependence of their porosity values. The blue curve represents the best fit, whereby the Kozeny-Carman constant $c_{1}$ is used as the only fitting parameter.
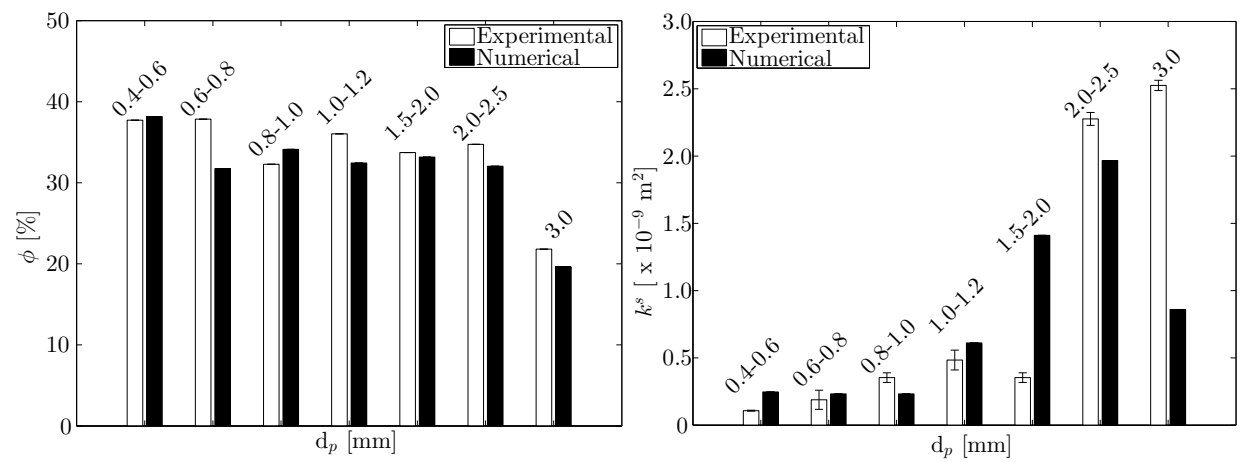

Figure 2.10: Numerically and experimentally determined porosity (left) and permeability (right) for sintered glass bead samples from different particle diameters. The experimentally determined permeability results represent the average value of five to eight independent measurements with the error bar representing the standard deviation of the measurements. The numerical results are obtained from subsets of 1024 voxel-sided cubes. The largest deviation between numerical and experimental permeabilities is observed for the glass bead sample with diameter $3.0 \mathrm{~mm}$. In this case, the representativeness of the investigated subset is not sufficient. 

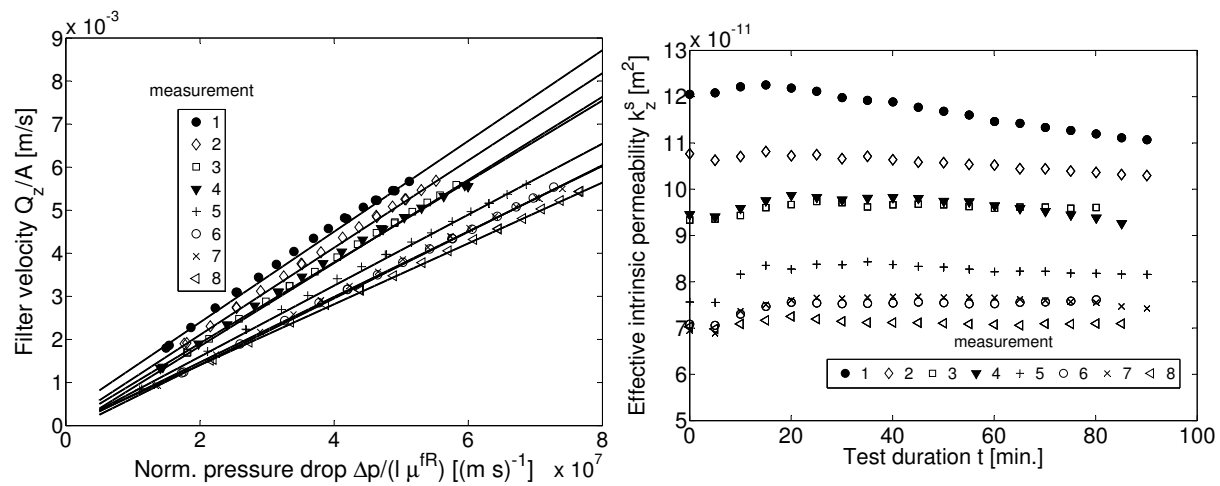

Figure 2.11: (Left) Filter velocity $Q_{z} / A$ as a function of pressure drop $\Delta p / l$ normalized by the real fluid viscosity $\mu^{f R}$. The permeability measurements are repeated 8 times for the sintered glass bead with diameters between 0.4 and $0.8 \mathrm{~mm}$. The almost linear relationship between the filter velocity and the normalized pressure drop confirms the applicability of Darcy's law and the stability of the experimental setup in a broader range of pressure gradients. (Right)Intrinsic permeability in dependence of test duration $t$. The intrinsic permeabilities of the different measurements show an almost constant behavior in dependence on the test duration for different volume fluxes. The small fluctuations are caused by uncertainties of the measuring equipment. For the sake of clarity, only each 150th measuring point in both figures is plotted. 


\subsubsection{Porosity and Permeability}

Figure 2.8 illustrates the numerical results of porosity and permeability values of extracted subsets with edge lengths of 256 voxel in each spatial direction obtained from $\mu \mathrm{XRCT}$ analysis of the sintered sample with bead diameters ranging between 0.4 and $0.6 \mathrm{~mm}$. In total, $4 \times 4 \times 8=128$ cubes are investigated in terms of porosity and intrinsic permeability. After evaluating the porosity and permeability values of the extracted subsets, the cubes with edge lengths of 256 voxels are arranged in compliance with their spatial coordinates within the initial cuboid depicted in Figure 2.6. For both, the porosity and permeability, a clear gradient with sample height can be observed. The permeability and porosity decrease with increasing depth due to the gravitational forces acting on the beads during sintering. The porosities vary only between 0.32 and 0.39 , whereas the permeability fluctuations within the initial cuboid are considerably higher $\left(1.29 \times 10^{-10}-2.75 \times 10^{-10} \mathrm{~m}^{2}\right.$, $)$, cf. Figure 2.8. In comparison, the effective porosity of the cylindrical specimen is experimentally determined from the bulk and bead densities by

$$
\phi_{0}=1-\frac{\rho^{s}}{\rho^{s R}}=37.72 \%,
$$

whereby the $\rho^{s}$ and $\rho^{s R}$ represent the bulk density and the effective true density of the beads, which the sintered sample is composed of. The experimentally determined effective porosity value lies within the porosity range determined from the segmentation of 256 voxel-sided cubes. The differences between experimentally determined porosities and voxel-based local porosities result especially from the spatial porosity gradient across sample height $z$. Since the phase-contrast between the porous skeleton composed of sintered glass beads and pore space is high, the influence of the segmentation procedure on the voxel-based porosity is assessed to be negligibly small. The permeability values at the top of the initial cuboid are more than twice greater than the permeabilities in the lower regions of the initial cuboid. The higher permeability fluctuations are confirmed in the conventional RVE analysis as shown in Figure 2.8 (bottom). The illustrations in Figure 2.8 (bottom) show the RVE analysis for porosity and permeability of differently sized subsets taken from the initial cuboid. Due to the varying degrees of fluctuations of porosity and permeability, the relative standard deviations related to the arithmetic mean value seem to be a sensible quantity to investigate the effect of the size of RVE edges. The percentage values in Figure 2.8 (bottom) show the relative standard deviations relative to the arithmetic mean values of the permeabilities being considerably higher than those of the porosities. For example, the standard deviation of porosity for cubes with edge length 256 voxel is only about $5 \%$, while the standard deviation of permeability is significantly higher at $17.77 \%$. This indicates that the permeability in general is more sensitive to the 
size of the representative volume element than the porosity. Furthermore, the findings from the RVE analysis confirm that the mean values for porosity and permeability are (almost) identical to that of the initial cuboid with dimensions of $1024 \times 1024 \times 2048$ voxel $^{3}$. A consequence of this result is that, either a few calculations of smaller sided cubes with low costs and computation time requirements can be performed or one cost intensive calculation on the initial cuboid with approximately 78,300 particles can be carried out.

Figure 2.9 (left) shows the permeabilities of the subsets as function of their porosities, for three different theoretical estimates, increasing according to KozenyCarman ${ }^{1}$. Figure 2.9 (right) shows the intrinsic permeabilities normalized by the square of the mean particle diameter, confirming the non-linearly increasing trend of the intrinsic permeability with increasing porosity. The colored data points in Figure 2.9 (left) represent the predictions according to the KozenyCarman model, c.f. Equation (2.3), whereby the arithmetic (red), harmonic (blue) and effective (black) diameter according to Equation (2.4) from the particle size distribution are used as representative values to predict the permeabilities of the differently sized subsets. The permeabilities determined from the lattice Boltzmann simulations (open data points) and the predictions according to Kozeny-Carman using the arithmetic mean diameter show a similar increasing trend, whereby the numerically determined permeabilities are higher by a factor of approximately 1.3. The permeability predictions according to Kozeny-Carman using the harmonic or effective diameter according to Equation (2.4) (blue and black data points) show stronger fluctuations and clearly underestimate the numerically determined permeabilities. Fitting the predicted permeability values depicted in red into numerical results by using the empirical constant $c_{1}$ as fit parameter, yields a value of 131, see Figure 2.9 (right). This constant contains and reflects the effect of the microstructure (particle shape, tortuosity) as a result of the sintering process on the intrinsic permeability of the glass bead samples.

Besides the porosity analysis on differently sized cubes extracted from different regions of the entire scanned region, subvolumes in the form of tubes with varying mean cross-section diameters are segmented to investigate the porosity distribution across the cross-section of the cylindrical sintered samples, cf. Figure 2.12 (state $\mathrm{C}$ ). To guarantee the investigation of meaningful representative volume elements, the pipe thickness is chosen as $3 \mathrm{~mm}$, which is equivalent to 187.5 voxels. Figure 2.12 (D.) shows the resulting porosity distribution in dependence on the mean pipe cross-section radius of the investigated specimens showing different glass bead diameters and degree of polydispersity. Starting from the center of the investigated samples, the porosities remain relatively constant up to a

\footnotetext{
${ }^{1}$ with second and third power of porosity
} 


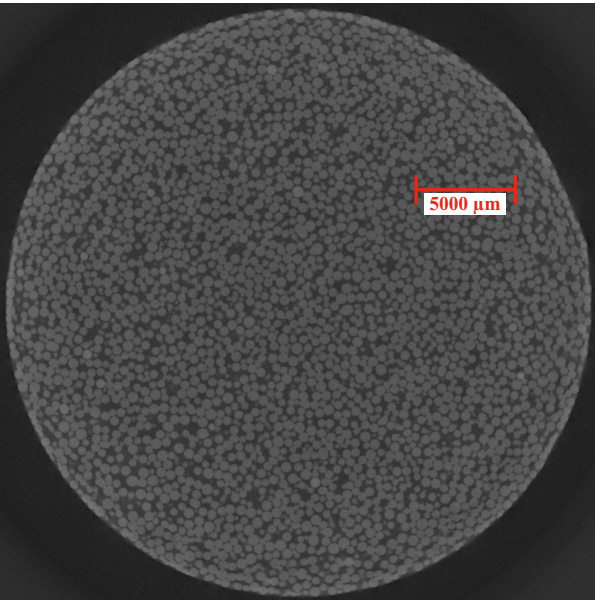

A.)

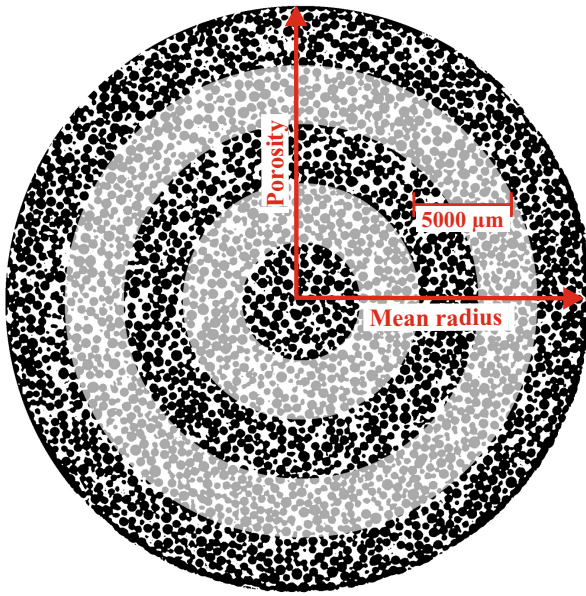

C.)

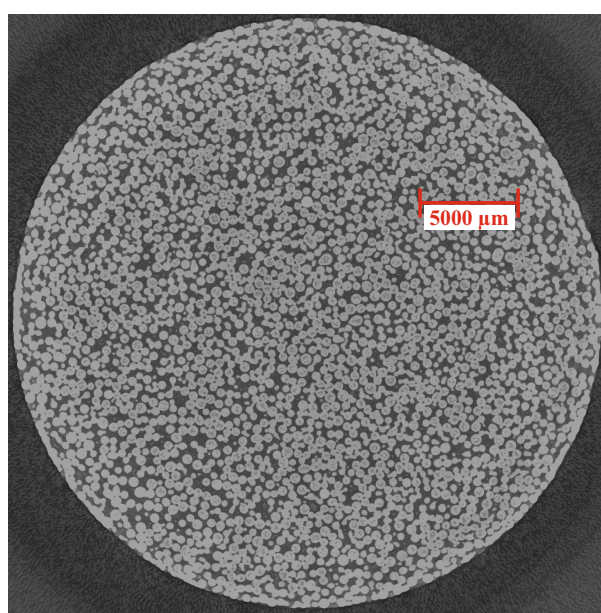

B.)

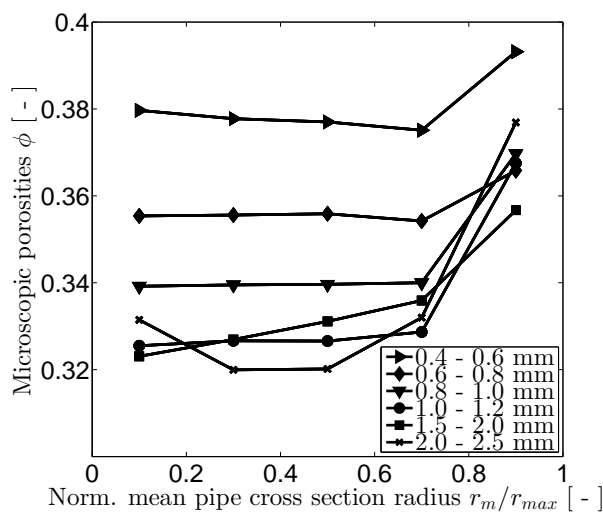

D.)

Figure 2.12: Cross section view of sintered specimen with glass bead diameter between 0.4 and $0.6 \mathrm{~mm}$ : A.) Raw data, B.) after filtering C.) after segmentation of tubes with different mean radii, and D.) porosity in dependence on the mean radial distance of the pipe cross-section from the center for different specimens featuring different glass bead diameters and degrees of polydispersity. 
mean cross-section radii of $11.5 \mathrm{~mm}$ and then increase to higher porosities for the largest investigated pipes in the external area of the produced samples. In these outer zones a clear increase of the porosity values for investigated samples can be seen due to the different contact between beads and cylinder wall during the sintering procedure and the gaps remaining between particles and walls. The clear porosity increase on the edges of the sintered samples is a consequence of the sintering procedure caused mainly by the presents of the walls.

Figure 2.11 summarizes the experimental results from permeability measurements for a sintered sample with bulk diameter of $30 \mathrm{~mm}$ showing glass bead diameter between 0.4 and $0.6 \mathrm{~mm}$. The measurements are repeated eight times in order to attain mean reliable results and understand the variation and reproducibility. The almost linear relationship between the filter velocity $Q_{z} / A$, and the pressure drop $\Delta p / l$, depicted in Figure 2.11 (left), and the nearly constant values of the effective intrinsic permeability $k_{z}^{s}$ over the test duration $t$, shown in Figure 2.11 (right), confirm the applicability of Darcy's law (2.1) and that the permeability measurements have taken place in the laminar Darcy regime. The measurement of water flow and pressure difference is captured with a sample frequency of $0.5 \mathrm{~Hz}$. For the sake of clarity, only each 150th measuring point is plotted in both figures. The intrinsic permeability of each measurement is determined from the slope of the linear regression lines, cf. Figure 2.11 (right). For the sintered sample with bead diameters ranging between 0.4 and $0.6 \mathrm{~mm}$, a mean effective permeability of $k_{z}^{s}=9.87 \times 10^{-11} \pm \Delta 1.65 \times 10^{-11} \mathrm{~m}^{2}$ is determined.

The bar diagrams in Figure 2.10 show a direct comparison between experimentally and numerically determined porosities and permeabilities of the investigated samples with different glass bead diameters and degrees of polydispersity. The numerical porosity and permeability results, represented by black bars, are obtained from 1024 voxel-sided cubes taken from the center part of the entire scanned regions of the samples and used in LB simulations. The effective permeability results obtained from laboratory experiments, represented by white bars, are averaged values from 5 (or 8 ) independent permeability measurements, whereby 10 to 15 different measuring ranges for volume fluxes and pressure differences are run during each measurement, cf. Figure 2.3. While the porosity values show a good agreement, the permeability results show much larger nonsystematic deviations. It should be noticed that small deviations in porosity and particle size can lead to significant deviations in permeabilities due their exponential influence, see Equation (2.3). Furthermore, it can be seen from Figure 2.10 (right) that the error bars in experimental permeability determinations are small compared to their averaged values, which indicates high reliability, repeatability and robustness of the used experimental permeability setup. 
Moreover, the good agreement between voxel-based local and experimental determined global porosity values of the investigated different sintered samples in Figure 2.10 (left) proves the correctness of the filtering and segmentation procedure despite the underlying porosity gradient across the sample height. A proper filtering and segmentation procedure is surely essential for subsequent permeability simulations. Incorrect porosity determination during segmentation procedure can lead to substantial errors in permeability calculations, see Equation (2.3). In this context sintered glass bead samples as simple replacement material for natural sandstones are suitable to set a benchmark for permeability calculations. Taking into account the porosity and permeability gradients with the sample height, the deviations between experimental and numerical results is insignificant. The maximum deviation factor of 2.4 that is obtained for glass bead diameters of $3.0 \mathrm{~mm}$ due to low representativity of the investigated subset is quite reasonable.

From the RVE analysis, we have come to the important conclusion that the average value for porosity and permeability of smaller sided cubes is (almost) identical to results obtained from the initial cuboid, cf. Figure 2.8. We have found out that the averaged values for permeability are almost equal independent from the size of the investigated subsets.

Furthermore, it became clear that a proper porosity determination from XRCT images is essential for numerical permeability calculations. A systematic error, for instance, during the filtering and segmentation procedure, can contribute to changes in porosity and morphology of the porous matrix, and therefore cause exponential errors in permeability calculations. If we are able to correct systematic errors during image processing, we obtain accurate permeability values and the trend in permeability is reproduced correctly, cf. Figure 2.9.

\subsubsection{Tortuosity}

Figure 2.13 (right) shows the distribution of the so-called Centroid Path Tortuosity within the initial cuboid according to Equation (2.7) determined from 2-dim slices of the XRCT data, see also Figure 2.13 (left) and Equation (2.7). It should be noted that tortuosity used in this study is a purely geometrical quantity, which is independent from the Re number. The problem of multiple definitions for tortuosity, which resulted in different findings, especially in the research field of poroacoustics, is reported in [47]. In contrast to the spatial porosity and permeability distributions, the (geometrical) tortuosity shows no significant vertical gradient with the sample height. The geometrical tortuosity ranges from 1.28 to 1.72 within the initial cuboid. Carman himself has estimated the hydraulic tortuosity of non-sintered glass beads at $T_{h y d r}=\sqrt{2}$ by injecting a dye into the sample $[40,51]$. The tortuosity value determined by Carman lies therefore within 
the tortuosity range determined from 2-dim slices of 256 voxel-sided cubes. Low values of tortuosity indicating less winded fluid paths are especially located at the top and bottom of the cuboid. Low tortuosity values in upper regions are caused by low porosity values, whereas low tortuosity values in deeper layers results from the higher connectivity of the pore channels. It might be quite possible that higher densification of the sample in deeper layers due to gravitational effects has lead to an increase of the coordination number of the glass particles positioned in lower regions, and simultaneously to an higher connectivity of pore channels. The higher mean coordination of the 256 voxel-sided cubes located at the bottom of the initial cuboid also reduces the tortuosity of the investigated cubes, since there is an increase in the number of available pathways from each glass beads to its neighbors. The increase in the number of available pathways has increased the possibility of connections which are closer to a straight path between two points. Similar observations were recently made in the investigation of the geometrical tortuosity in sphere and ellipsoid packings by Stenzel et al. [174]. It can be assumed that the determined tortuosity values depend besides porosity also from the mean coordination number of the investigated subsets.

However, the (geometrical) tortuosity is not decisive for the numerical intrinsic permeabilities of the investigated subsets, rather, they are determined by the sizes pore spaces, pore throats and porosities, cf. subsection 2.6.1 Porosity and Permeability and subsection 2.6.4 Pore throats. The independence of the intrinsic permeability from the geometrical tortuosity is also confirmed in Figure 2.14 (right). In addition to the permeability, there is also no correlation between porosity and geometrical tortuosity, see Figure 2.14 (left). It can be noticed, that the computed tortuosities obtained from the $\mu \mathrm{XRCT}$ data analysis are overestimated by theoretical predictions according to Berryman [21, 193], cf. Equation (2.6). It can be clearly seen that the Berryman model is not sufficient to describe the tortuosity in sintered glass beads packages. For a more sophisticated model, the sintering effects and the associated increase of the pore channel connectivity due to densification of the green body have to be taken into account.

\subsubsection{Sphericity and specific surface area}

Figure 2.15 (top left) illustrates the spatial local distribution of mean sphericity, defined in Equation (2.13) and obtained from particles in 256 voxel-sided cubes within the initial cuboid. It can be seen that smaller sphericity values indicating larger deformations can be found in deeper layers due to the gravitational effects. Figure 2.15 (bottom left) shows the sphericity distribution after sintering within the initial cuboid containing approximately 78,300 particles. Most of the particles show sphericity around 0.96 . The sphericity values near 1 and the relative high 

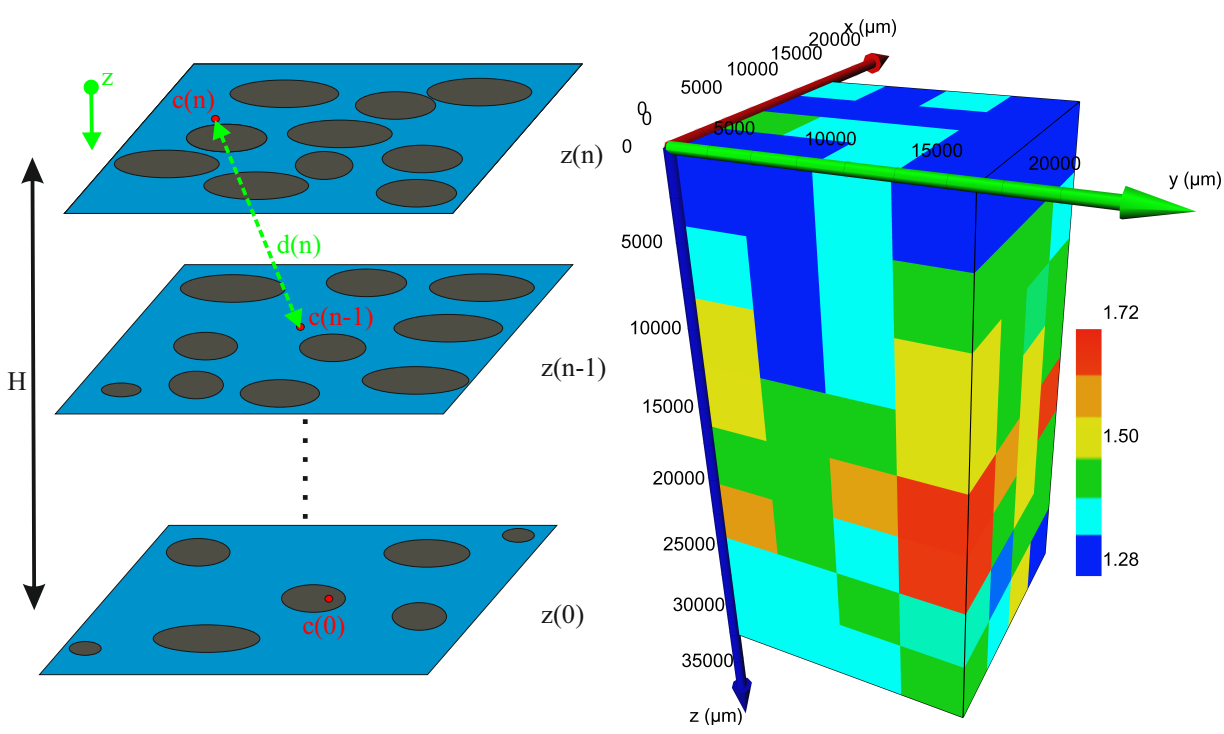

Figure 2.13: Method of determining the so-called centroid path tortuosity from binarized 2D slices of the CT image (left). Distribution of tortuosity within the initial cuboid with the dimensions of $1024 \times 1024 \times 2200$ voxel $^{3}$. The geometrical tortuosities are determined from cubes with dimensions 256 voxels in each spatial direction and assigned according to their spatial coordinates (left).
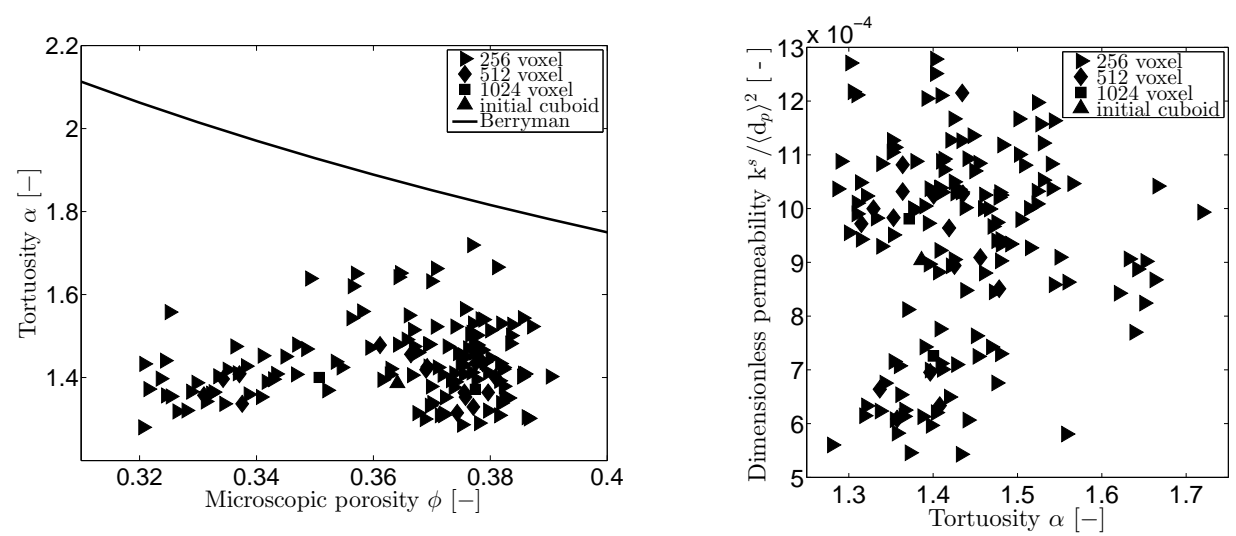

Figure 2.14: Centroid path tortuosities $\alpha$ of differently sized subsets taken from the initial cuboid as a function of porosity $\phi_{0}$ (left). Intrinsic permeabilities $k_{z}^{s}$ plotted against the Centroid Path Tortuosity $\alpha$. 
porosity around $35 \%$ indicate that the microstructure of the produced samples is predominantly formed in the first and intermediate sintering stage where point contacts between the glass beads dominate.

Moreover, the distribution of the relative standard deviation of the specific volumebased surface area related to the minimum specific surface obtained from the equivalent particle diameter shows a peak at only 3.7\%, cf. Figure 2.15 (bottom right). These factors indicate that the sintered glass beads have not lost much of their original spherical shape and the sphere-based Kozeny-Carman equation can be applied to predict the intrinsic permeability of the sintered glass bead packing. Sphericity values $\psi_{s}<0.9$ and relative standard deviations of the specific surface area $S_{v}-S_{v, \text { min }} / S_{v, \text { min }}>8 \%$ can be clearly assigned to glass beads which are positioned in the outer regions of the initial cuboid.

The mean specific surface area gained from 256 voxel-sided cubes decreases from top to bottom like the intrinsic permeability, cf. Kozeny-Carman Equation (2.2) and Figure 2.15 (top right). The lower values of the mean specific surface in deeper layers is caused by the higher densification of the sample due to gravitational effects. The porosity decrease towards deeper layers and the decrease of the specific surface area lead to lower permeabilities in deeper regions.

\subsubsection{Pore throats}

In addition to porosity, specific volume-based surface area, sphericity and particle size, the intrinsic permeability in sintered glass samples is also affected by pore throats [175]. The strong influence of pore throats in simple pore systems, consisting of cylinder pores, on the intrinsic permeability can be derived analytically, cf. Ref. [116]. Numerous statistical models for determination of pore throat distribution from particle size distributions of granular packings exist, cf. Ref. [90, 158, 178]. However, the applied models are often inaccurate to determine the constriction size distribution. In addition, the direct link to the intrinsic permeability is missing. Furthermore, the influence of the sintering process remains unconsidered. In such cases, the $\mu \mathrm{XRCT}$ analysis provide an efficient tool for visualization and quantification of pore throat areas and to investigate their impact on the intrinsic permeability [177]. Therefore, this section presents a simple methodology for the determination of the pore throat areas from $\mu \mathrm{XRCT}$ data. Moreover, the correlations between the mean pore throat diameter $\left\langle\mathrm{d}_{p t}\right\rangle$, the porosity $\phi_{0}$ and the numerical determined intrinsic permeabilities are investigated in detail.

Figure 2.16 (left) illustrates the local distribution of mean pore throat diameter from 256 voxel-sided cubes within the cuboid. The normalized pore throat distribution gained from the initial cuboid in Figure 2.16 (right) depicted in red shows 


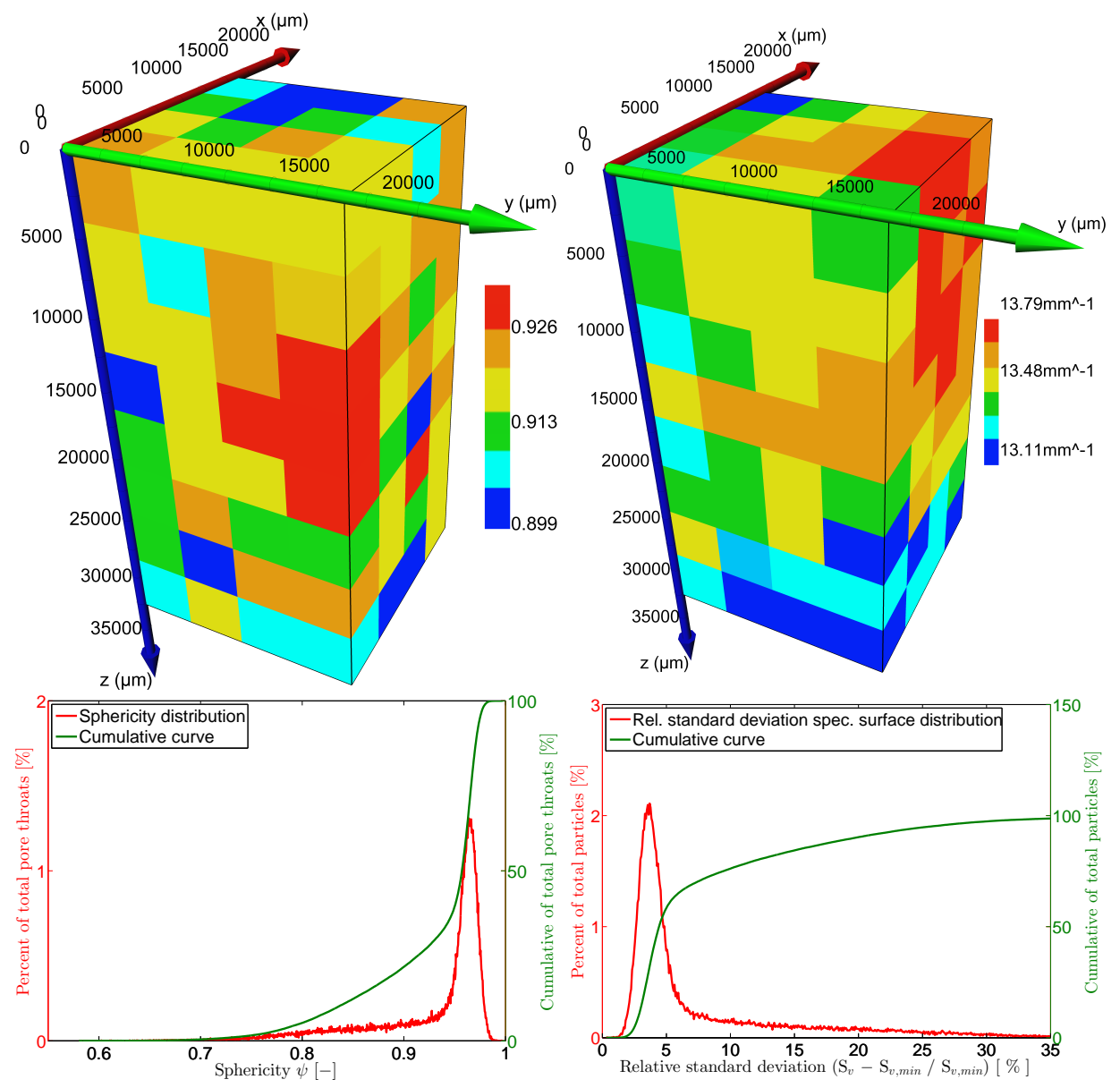

Figure 2.15: Local distribution of numerical determined mean sphericity and specific surface values of subsets with edge lengths of 256 voxels within the initial cube. Distributions for sphericity and relative standard deviation of specific surface related to the minimum specific surface obtained from equivalent particle diameter are shown below (red curves). The corresponding cumulative curves are depicted in green. 

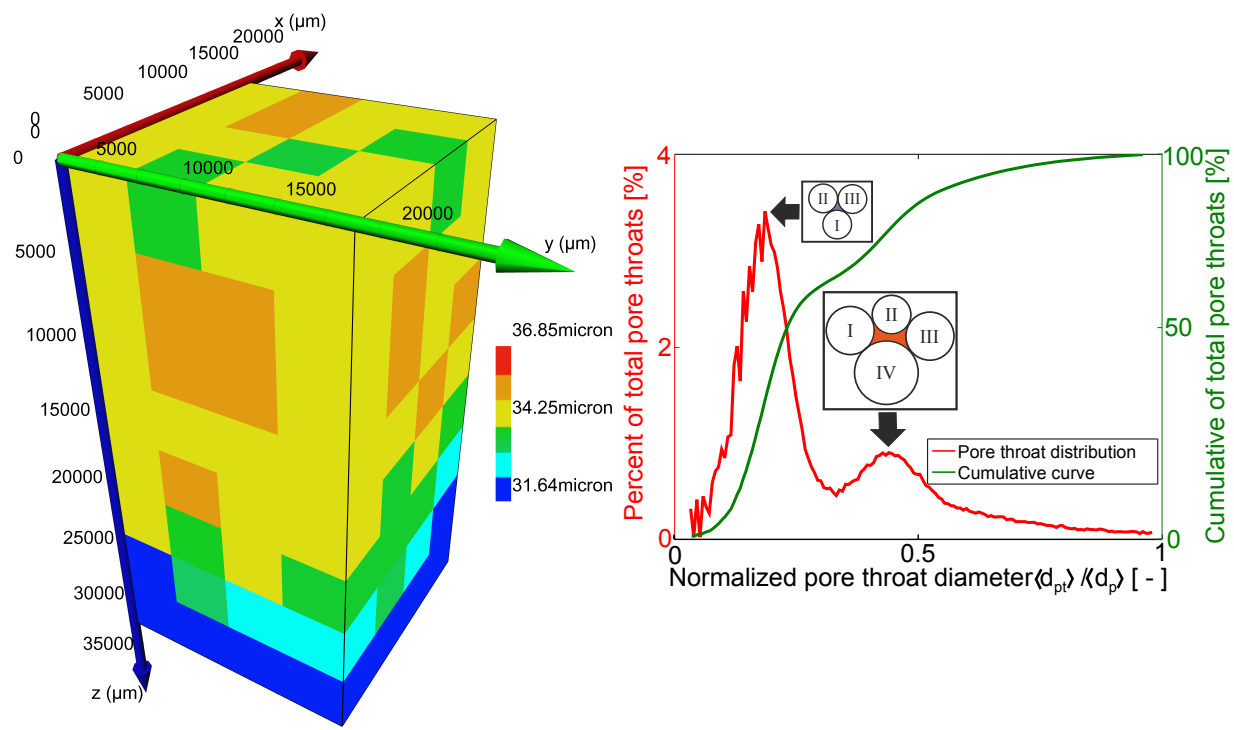

Figure 2.16: Local spatial distribution of mean pore throat diameter values of subsets with edge lengths of 256 voxels within the initial cuboid (left). Frequency distribution for equivalent mean pore throat diameter $\left\langle d_{p t}\right\rangle$ (red curve) and the corresponding cumulative curve (green curve) (right).

two distinct peaks which can be clearly assigned to pore throat areas formed by either three or four particles. The larger peak at smaller diameters results from pore throat areas which are formed by three particles whereas the smaller peak at larger diameters is due to four-particle-constellations. The probability of occurrence of pore throats formed by three particles is certainly higher compared to pore throat areas resulting from four-particle-constellations. Downscaling to smaller volume elements (up to 256 voxel-sided cubes) showed qualitatively the same pore throat distributions. Depending on the degree of polydispersity of the investigated sample (cf. Table 2.1) and the sintering durations the pore throat distributions showed different width of the peaks. In accordance with local distributions for porosity and permeability, the equivalent mean pore throat diameter also shows a spatial gradient along the sample height $z$, see Figure 2.16 (left), decreasing from top to bottom about $14 \%$ due to compaction in deeper layers.

The correlation of the permeability, the porosity and the mean pore throat diameter is plotted in Figure 2.17, displaying the porosities $\phi_{0}$ (left) and the normalized intrinsic permeabilities $k_{z}^{s} /\left\langle d_{p}\right\rangle^{2}$ (right) of differently sized subsets taken 

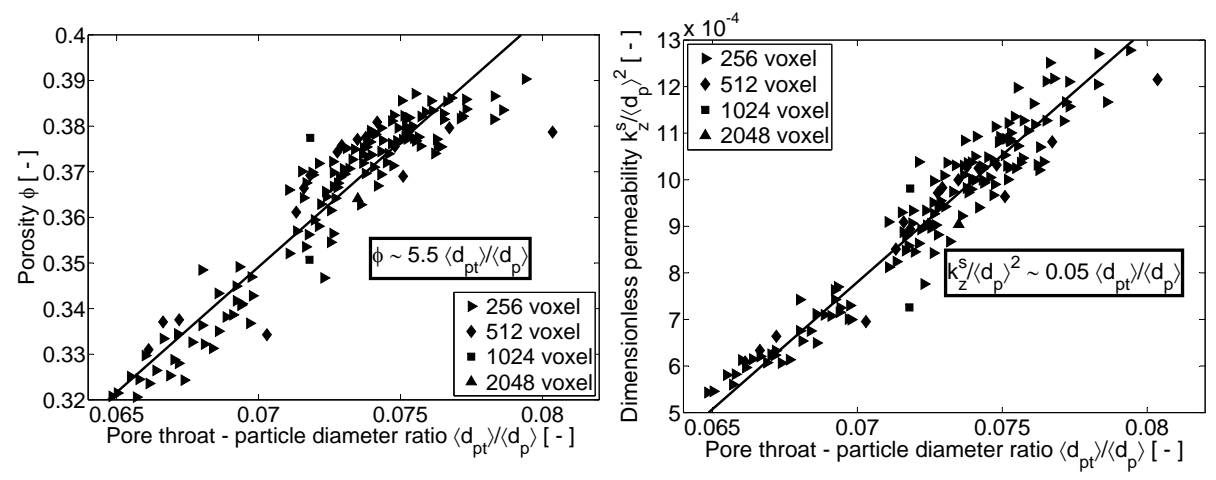

Figure 2.17: Porosity (left) and dimensionless permeability (right) in dependence on the mean pore throat diameter normalized to the mean particle diameter. Both, the porosity $\phi_{0}$ and normalized intrinsic permeability $k_{z}^{s} /\left\langle d_{p t}^{2}\right\rangle$, show linear dependency on the normalized mean pore throat diameter $\left\langle d_{p t}\right\rangle /\left\langle d_{p}\right\rangle$.

from the initial cuboid in dependence on their normalized mean pore throat diameters $\left\langle d_{p t}\right\rangle /\left\langle d_{p}\right\rangle$. For both, the porosity and the normalized permeability a clear linear dependency on the normalized mean pore throat diameter $\left\langle d_{p t}\right\rangle /\left\langle d_{p}\right\rangle$ can be seen, with correlation coefficients of the fits of 0.9378 and 0.9675 , respectively. The higher mean pore throat diameters correlating with the higher porosity and the higher permeability values are located at the top of the investigated cuboid and decrease towards deeper layers.

\subsection{Conclusion}

In summary, we have demonstrated that for a comprehensive evaluation of hydraulic properties of sintered glass beads a holistic consideration of the material properties porosity, tortuosity, sphericity, specific surface and pore throats is required. In this respect, the non-destructive X-ray Computed Tomography is an efficient tool, which enables the visualization and quantification of internal (microstructural) parameters including the (voxel-based) morphometry of the porous sample. The way $\mu \mathrm{XRCT}$ data are processed is crucial. In this study, different ways have been introduced in order to identify the essential control parameters for the hydraulical properties of the sintered glass bead systems. The presented framework can in future be used to evaluate also the hydraulical characteristics of other kinds of porous media.

The main findings of this study can be summarized as: 
- The intrinsic permeability in sintered granular packings depends not only on the porosity, but also correlates with the equivalent pore throat mean diameter.

- The pore throat distributions in slightly polydisperse packings show two distinct peaks arising from typical three- or four-particle constellations.

- In the given narrow porosity and permeability range, a linear dependency of the permeability with the equivalent pore throat diameter is observed.

- The quantitative comparison between experimental and numerical permeability values requires a proper filtering and segmentation procedure, and then leads to good agreement.

For the examined sintered glass bead samples a clear spatial gradient of microscopic porosity, permeability and mean pore throat diameter along the sample height could be detected as a result of gravitational compaction during sintering. Local RVE analysis has revealed that the averaged values for porosity and intrinsic permeability of smaller sided cubes are (almost) identical to results of larger subsets obtained from the cuboid. A consequence of this result is that, we can either perform many cost effective and time-saving computations on small subsets, which are less representative, or compute one cost and time intensive large subset, which is more representative. For the calculations of effective quantities new strategies can be developed on the basis of results gained from the RVE analysis.

From the RVE data, the porosity development across the radial direction was analyzed. A slight increase of porosity at peripheral outer zones could be identified due to the contacts of the beads with the cylinder wall during sintering. Rotational sintering under pressure-controlled gas atmosphere may eventually lead to more homogeneous samples avoiding spatial gradients of porosity and permeability. Substantial effort is required to produce homogenous samples. In commercial available sintered samples so-called "raining devices" are used to avoid the sedimentation of the beads. The beads are filled in a copper cylinder and shaked for consolidation and finally saturated with water. The green compact is sintered under fixed stress and homogenous temperature distribution within the furnace. Note that in this case only the center part of the samples is homogenous, which shows that the production of homogenous samples is in general very challenging. From the experimental point of view, the presence of air bubbles in the measuring cell poses a big challenge, which can highly affect the measurement of pressure differences, and thus of the intrinsic permeability. Taking into account these difficulties, a comparison between numerical and experimental determined intrinsic permeability values gives us the possibility to better understand the system. Eventually, we have achieved qualitatively and quantitatively a good 
agreement between experiment, microstructural analysis and numerical simulations.

We have demonstrated through 3-dim LB simulations that a proper filtering and segmentation procedure during $\mu \mathrm{XRCT}$ analysis is essential for porosity determinations and to obtain accurate permeability results. We have found that an almost linear correlation between both intrinsic permeability and porosity with the pore throat equivalent diameter can be observed if systematic errors during filtering and segmentation are avoided. Our numerical and experimental permeabilities are in general agreement with permeability predictions according Kozeny-Carman, although the validity of the Kozeny-Carman equation is limited to monodisperse and non-sintered granular packings. In contrast to $c_{1}=180$ for idealized sphere packings, a value of $c_{1}=131$ for the empirical constant, which takes into account microstructural effects resulting from the sintering treatment of the polydisperse glass bead packing, could be determined by fitting the predicted permeability values to numerical results from LB simulations. Moreover, sphericity values around 0.95 confirm the low deformation during sintering and thus, the glass beads have not lost much of their original shape.

We have made the observation that the numerical determined local permeabilities are mainly influenced by pore throats and local porosity values and less by mean specific surface, tortuosity and mean sphericity.

In this study XRCT structural and permeability analysis have been performed for several slightly polydisperse sintered glass bead samples with different primary particle average diameters. The observed micro-macro relations scale with the average diameters of the rather large particles. Further work is needed to extend the study towards systems with smaller particles, higher polydispersity or systems with longer sintering duration time and thus lower porosity. It has to be seen if the presented approach for the processing of the XRCT data and the resulting main findings, for instance the linear dependency of the intrinsic permeability with the pore throats, can be also found in those systems, and in natural porous materials like sandstone.

\subsection{Acknowledgement}

We acknowledge support from the Stichting voor Fundamenteel Onderzoek der Materie (FOM), which was financially supported by the "Nederlandse Organisatie voor Wetenschappelijk Onderzoek" (NWO), (project number: 09iPOG14-1/2). We also thank Matthias Halisch from the Leibniz Institute for Applied Geophysics (LIAG) in Hannover, Germany for performing and providing $\mu$ XRCT scans, as well as the Jülich Supercomputing Centre for providing the CPU time for the lattice Boltzmann simulations. 


\section{CHAPTER 3}

\section{Oscillatory Fluid Flow through Artificially Produced Porous Media}

An experimental program for so-called pore pressure oscillation test is described and experimental results for the hydraulic response of fluid-saturated artificial samples consisting of sintered glass bead packages and synthetic samples consisting of capillary tubes is presented. Frequency-dependent amplitude ratios and phase differences of the pressure signals are analyzed in order to determine the frequency-dependent hydraulic properties, such as the dynamic permeability, storage capacity and diffusivity of the produced samples. We show that the pore pressure oscillation test, which is commonly used for "tight" rocks, can be applied to high-permeable artificially produced samples with permeabilities in the order of $10^{-13}-10^{-9} \mathrm{~m}^{2}$. The advantages and disadvantages of the pore oscillation method are shown and discussed.

\subsection{Introduction}

The hydro-mechanical response of a fully fluid-saturated porous medium to an oscillatory pressure change is determined by two major factors, namely permeability and storage capacity $[150,168,169]$. While the permeability is a measure of the ability of the porous sample to pass a fluid, when a pressure gradient between the ends of sample is applied, the storage capacity is a measure of the fluid 
storage by relating the change in fluid volume and pore pressure present in the pore space $[103,151,168]$. The storage capacity depends on the hydro-mechanical response of the fluid-filled porous medium. Both parameters are highly influenced by the underlying micro- and macrostructure of the porous material. Different commonly used standard techniques and models for permeability determinations (e.g. the constant pressure difference method, cf. Figure 3.1), which partially rely on the measurement of the volumetrical flux and simplifications, are often limited by certain parameter ranges and accuracy $[55,56,168]$. In this connection, the applicability of the standard techniques is additionally limited by technical restrictions of the measuring devices. Problems relating the measuring of the volumetrical flux of low-permeable rocks $[31,113,168]$ or measuring the static pressure difference of high-permeable materials are reported elsewhere [168].

In this case, the so called pore pressure oscillation method, which is based on the analysis of sinusoidal pressure signals positioned at the up- and downstream reservoir of the sample is more than an effective alternative tool to determine the hydraulic properties of a porous material. An important advantage of the pore oscillation method is that the pressure development in pore space can be selectively influenced in terms of amplitude and frequency. In this sense, the pore oscillation method takes into account the frequency dependence of the measured hydraulic parameters, wheres the constant pressure method or transient pulse decay tests are insensitive to any frequency dependance of the measured hydraulic parameters [55].

For comparison Figure 3.1 illustrates simplified sketches of the commonly used constant pressure difference method for permeability determination (A.) and the pressure oscillation method (B.). For the pressure oscillation method, a controlled and fixed-frequency oscillation with a fixed pressure amplitude in the upstream reservoir is used, which finally results in decayed and phase-shifted pressure oscillation in downstream reservoir after propagating through the porous sample. The amplitude ratio and the phase difference between the pressure signals of the upand downstream reservoir provide information about the hydraulical properties of the porous sample [102]. In this study, we demonstrate that the pore oscillation method can be applied successfully on high-permeable artificial samples with macroscopic global permeability values in the order of $10^{-13}-10^{-9} \mathrm{~m}^{2}$ and macroscopic porosity values ranging between $0.01 \%$ and $36.79 \%$. Besides the investigations of sintered glass bead samples with different glass bead diameters, synthetic samples with simple pore geometry consisting connected capillary tubes in series or parallel are tested in pore pressure oscillation tests. The hydraulic properties of the investigated samples are analyzed in a wide frequency range between 0.01 and $1000 \mathrm{~Hz}$. In addition to frequency sweeps, amplitude sweeps tests are performed, in order to assess the influence of non-linearity effects on the performed pore oscillation tests. Furthermore, different set-up configurations, 


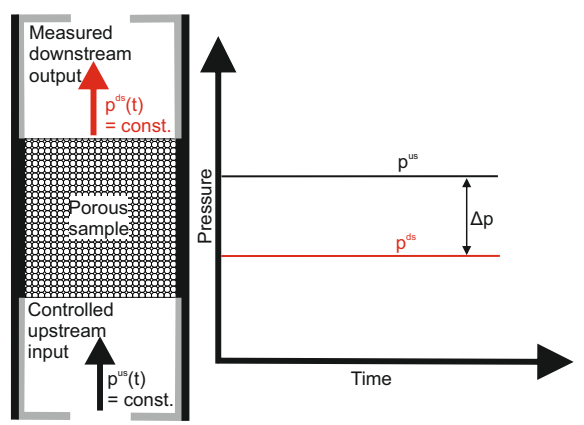

A.)

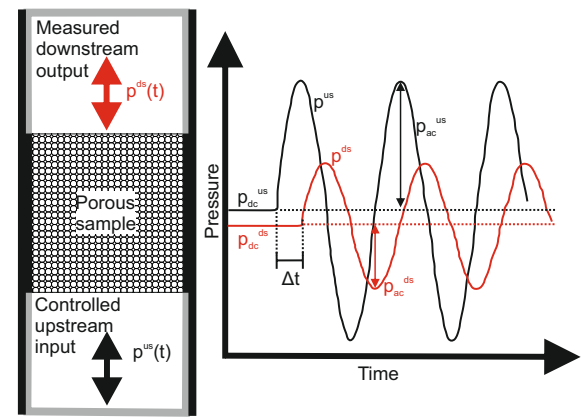

B.)

Figure 3.1: Sketch of different experimental set ups for permeability determination with corresponding pressure curves: A.) Constant pressure difference method, B.) Pressure oscillation method.

like the influence of different water levels in the downstream reservoir or several valve positions in the downstream reservoir on the pore pressure oscillation test are shown and discussed. In chapter 3.2 the theoretical and mathematical framework for dynamic permeability measurements are presented and the dynamic permeabilities of the investigated samples are determined according to the well-known Johnson-Koplik-Dashen-model (JKD model) [95] and discussed. Moreover, the pressure-diffusion equation for different cases (static, dynamic, time and frequency domain) is presented and discussed in terms of hydraulic parameters. In the following chapter 6.3 the experimental set-up for the pore pressure oscillation is described. Moreover, the experimental results of the investigated samples are presented and discussed. The final chapter 5.4 contains the conclusion regarding the application of the pore pressure oscillation method on high-permeable porous media.

\subsection{Theoretical background}

\subsubsection{Governing set of equations and JKD model}

The concept of dynamic behavior of permeability in porous media has already been described by Biot [24, 25, 27], when he formulated the field equations, which describe the acoustic wave propagation in fully-saturated poro-elastic media. Based on Biot's formulation of the momentum balance of the fluid, which 
is described in terms of primary displacement of the solid and fluid phase, we obtain according to [172]

$$
\rho_{12} \ddot{\mathbf{u}}_{s}+\rho_{22} \ddot{\mathbf{u}}_{f}-b_{0} F\left(\dot{\mathbf{u}}_{s}-\dot{\mathbf{u}}_{f}\right)=Q \operatorname{grad} \operatorname{div} \mathbf{u}_{\mathbf{s}}+R \operatorname{grad} \operatorname{div} \mathbf{u}_{f},
$$

whereby $\rho_{12}$ and $\rho_{22}$ describe the densities of the inertial terms, $b_{0}$ and $F$ the damping and correction factor, which characterizes the transition from viscous dominated flow regime to inertial dominated flow regime. In Equation (3.1) the elastic coefficients of the bulk medium are introduced by $Q$ and $R$ and the fluid and solid displacements as $\mathbf{u}_{s}$ and $\mathbf{u}_{f}$. However, for the introduction of the basic concept of dynamic permeability in biphasic mixtures, we assume a rigid porous medium with $\mathbf{u}_{\mathbf{s}}=\mathbf{v}_{\mathbf{s}}=0$, which simplifies Equation (3.1) to

$$
\rho_{22} \ddot{\mathbf{u}}_{f}+b_{0} F \dot{\mathbf{u}}_{f}=R \operatorname{grad} \operatorname{div} \mathbf{u}_{f}
$$

Inserting the constitutive relation for the pore pressure

$$
\operatorname{div} \mathbf{u}_{f}=\frac{p_{0}-p}{K^{f}}
$$

and the elastic Biot coefficient

$$
R=\frac{\phi^{2} K^{f}}{\phi_{0}} \quad \text { with } \phi_{0}=\phi \quad \text { and mass coefficient } \quad \rho_{22}=\alpha \phi_{0} \rho^{f R}
$$

leads finally to

$$
\alpha \phi_{0} \rho^{f R} \dot{\mathbf{w}}_{f}+b_{0} F \mathbf{w}_{f}=-\phi_{0} \operatorname{grad} p,
$$

whereby the seepage velocity is introduced as $\mathbf{w}_{\mathbf{f}}=\mathbf{v}_{\mathbf{f}}-\mathbf{v}_{\mathbf{s}}=\mathbf{v}_{\mathbf{f}}$. Furthermore, it is assumed that the effective porosity $\phi$ corresponds to initial porosity $\phi_{0}$ due to assumption of a rigid porous medium. The tortuosity of the pore channels is described by $\alpha$. Equation (3.5) demonstrates that the original field Equation (3.1) is constrained to a purely hydraulical problem.

For the transformation of the time-dependent hydraulic problem to the frequency domain an harmonic approach for fluid pressure and velocity is used

$$
p(\mathbf{x}, \omega)=\hat{p}(\mathbf{x}, \omega) \exp (-i \omega t) \quad \text { and } \quad \mathbf{w}_{\mathbf{f}}=\hat{\mathbf{w}}_{\mathbf{f}}(\mathbf{x}, \omega) \exp (-i \omega t),
$$

with frequency- and location-dependent amplitudes $\hat{p}(\mathbf{x}, \omega)$ and $\hat{\mathbf{w}}_{\mathbf{f}}(\mathbf{x}, \omega)$. Inserting the harmonic approaches for fluid pressure and displacement in Equation (3.6) and the initial damping factor

$$
b_{0}=\frac{\eta^{f R} \phi_{0}^{2}}{k_{z}^{s}}
$$


in Equation (3.5), leads finally to a frequency-dependent solution for the pressure gradient

$$
-\operatorname{grad} \hat{p}=\frac{\eta^{f R} \phi_{0}}{k_{z}^{s}(\omega)} \hat{\mathbf{w}}_{f}=\frac{\eta^{f R}}{k_{z}^{s}(\omega)} \hat{\mathbf{q}}=\left[\frac{\eta^{f R} \phi_{0}}{k_{z}^{s}} F(\omega)-i \omega \alpha \rho^{f R}\right] \hat{\mathbf{w}}_{f}
$$

with $\hat{\mathbf{q}}=\hat{\mathbf{Q}} / A$ as specific volumetric flux normalized to the cross sectional area of the porous sample and $\rho^{f R}$ and $\eta^{f R}$ as real density and viscosity of the pore fluid. In Equation (3.8), the frequency-dependent permeability in flow direction $z$ is introduced as

$$
k_{z}(\omega)=\frac{k_{z}^{s}}{F(\omega)-i \omega \frac{\alpha \rho^{f R} k_{z}^{s}}{\eta^{f R} \phi_{0}}}
$$

Inserting the critical frequency

$$
\omega_{\mathrm{crit}}=\frac{\eta^{f R} \phi_{0}}{\alpha \rho^{f R} k_{z}^{s}}
$$

and the frequency-dependent viscous correction factor as

$$
F(\omega)=\sqrt{1+0.5 i M \frac{\omega}{\omega_{\text {crit }}}}
$$

in Equation (3.9), the normalized dynamic permeability can be formulated as

$$
\frac{k_{z}(\omega)}{k_{z}^{s}}=\left[\sqrt{1+0.5 i M \frac{\omega}{\omega_{\text {crit }}}}+i \frac{\omega}{\omega_{\text {crit }}}\right]^{-1},
$$

whereby $M$ represents the shape factor of the pores and can be related to hydraulic and microstructural parameters as follows

$$
M=\frac{8 \alpha k_{z}^{s}}{\phi_{0} \Lambda^{2}}
$$

In Equation (3.13) $\Lambda$ is defined as the pore volume to pore surface ratio and $k_{z}^{s}$ is the intrinsic permeability of the porous medium. $\alpha, k_{z}^{s}$ and $\Lambda$ are independently measurable parameters. The shape factor is usually taken as $M \approx 1$ $[100,166,172]$. Equation (3.12) is known as the Johnson-Koplik-Dashen model (JKD model) [95] and is often used to model the dynamic behavior of permeability in porous media. It can be stated out consequently that the dynamic permeability depends only on four parameters $\alpha, k_{z}^{s}, \Lambda$ and $\phi_{0}$, which are well defined for any porous medium [95]. The critical frequency $\omega_{\text {crit }}$, formulated in Equation 3.10 separates the viscous-dominated flow regime from the inertial- 
dominated flow regime, which leads to a decrease of the dynamic permeability $k_{z}(\omega)$.

It should be noticed, that the JKD-model in Equation (3.12) takes the classical form of Darcy's law for $\omega \rightarrow 0$ and approaches zero for $\omega \rightarrow \infty$.

$$
\lim _{\omega \rightarrow 0} F=1, \quad \lim _{\omega \rightarrow \infty} F=\infty, \quad \lim _{\omega \rightarrow 0} k_{z}^{s}(\omega)=k_{z}^{s}, \quad \lim _{\omega \rightarrow \infty} k_{z}^{s}(\omega)=0,
$$

According to Johnson et al. [95] the dynamic permeability of a simple porous sample consisting of a tube with a radius $r_{t}$ and length $L$, whose axes form an angle $\theta$ with the direction of the applied pressure gradient can be formulated exactly as followed

$$
k_{z}(\omega)=\frac{\phi_{0} \eta^{f R}}{i \omega \rho^{f R}}\left[\frac{2 J_{1}\left(K r_{t}\right)}{K r_{t} J_{0}\left(K r_{t}\right)}-1\right] \cos \theta,
$$

whereby the $J_{0}$ and $J_{1}$ are first-type Bessel functions of zero and first order. $K$ is the shear wave vector and can be expressed in terms of the frequency-dependent viscous skin depth $\delta$

$$
K=\frac{1+i}{\delta} \quad \text { with } \quad \delta=\sqrt{\frac{2 \eta^{f R}}{\omega \rho^{f R}}}
$$

as the frequency-dependent viscous skin depth, which approaches zero for $\omega \rightarrow \infty$. The corresponding volumetric flow rate through the tube with a length $L$, which is imposed on sinusoidally varying pressure drop reads as

$$
\hat{Q}=\frac{\pi r_{t}^{2}}{i \omega \rho^{f R}}\left[\frac{2 J_{1}\left(K r_{t}\right)}{K r_{t}}-1\right] \frac{\operatorname{grad} p}{L} .
$$

The general parameters for the specific case of tube-shaped pores are given by

$$
\alpha=\frac{1}{\cos \theta}, \quad k_{z}^{s}=\frac{1}{8} \phi_{0} r_{t}^{2} \cos \theta, \quad \Lambda=r_{t} .
$$

For tube-shaped pore channels with different radii connected in parallel or in series the general effective parameters for the JKD model [95] are formulated as

$$
k_{z}^{s}=\frac{1}{8} \phi_{0} \frac{\left\langle r_{t}^{4}\right\rangle}{\left\langle r_{t}^{2}\right\rangle}, \quad \alpha=1.0 \quad \Lambda=\frac{\left\langle r_{t}^{2}\right\rangle}{\left\langle r_{t}\right\rangle}, \quad M=\frac{\left\langle r_{t}^{4}\right\rangle\left\langle r_{t}\right\rangle^{2}}{\left\langle r_{t}^{2}\right\rangle^{3}} \quad \text { and }
$$


Table 3.1: Input parameters for the investigated porous sintered glass bead samples.

\begin{tabular}{lcccccc}
\hline Parameter & Unit & $\mathrm{S} 1$ & $\mathrm{~S} 2$ & $\mathrm{~S} 3$ & $\mathrm{~S} 4$ & $\mathrm{~S} 5$ \\
\hline \hline Particle diameter $d_{p}$ & {$[\mathrm{~mm}]$} & $0.4-0.6$ & $0.6-0.8$ & $0.8-1.0$ & $1.0-1.2$ & 3.0 \\
Intr. permeability ${ }^{[A]}$ & {$\left[\mathrm{m}^{2}\right]$} & $1.96 \mathrm{E}-10$ & $4.66 \mathrm{E}-10$ & $3.19 \mathrm{E}-10$ & $6.82 \mathrm{E}-10$ & $1.26 \mathrm{E}-9$ \\
$k_{z}^{s}$ & & & & & & \\
Effect. porosity ${ }^{[B]} \phi_{0}$ & {$[\%]$} & 35.11 & 36.79 & 29.48 & 32.32 & 22.31 \\
Shape factor $M$ & {$[-]$} & 1 & 1 & 1 & 1 & 1 \\
Tortuosity ${ }^{[C]} \alpha$ & {$[-]$} & 1.46 & 1.47 & 1.51 & 1.56 & 1.86 \\
Crit. frequency ${ }^{[C]} f_{c}$ & {$[\mathrm{~Hz}]$} & 195.14 & 88.78 & 97.50 & 48.42 & 15.10 \\
\hline \hline
\end{tabular}

$\overline{[A]}$ : Determined from classical Darcy experiments.

${ }^{[B]}$ : Determined from bulk density of the produced samples

${ }^{[C]}$ : Obtained from 1024(x)X1024(y)X2048(z) cuboids extracted from $\mu \mathrm{CT}$ data

$$
k_{z}^{s}=\frac{1}{8} \frac{\phi_{0}}{\left\langle r_{t}^{2}\right\rangle\left\langle r_{t}^{-4}\right\rangle}, \quad \alpha=\left\langle r_{t}^{2}\right\rangle\left\langle r_{t}^{-2}\right\rangle \quad \Lambda=\frac{\left\langle r_{t}^{-2}\right\rangle}{\left\langle r_{t}^{-3}\right\rangle}, \quad M=\frac{\left\langle r_{t}^{-3}\right\rangle^{2}}{\left\langle r_{t}^{-4}\right\rangle\left\langle r_{t}^{-2}\right\rangle} .
$$

The input parameters for the investigated sintered samples and for the synthetic samples consisting of tube-like pore channels used for prediction of the frequency-dependent permeabilities according to the JKD model are summarized in Table 3.1 and 3.2 .

From Equation (3.12) it can be seen, that the dynamic permeability is complexvalued. Figures 3.2 and 3.3 show the absolute values (A.), the phases (B.), the real (C.) and the imaginary part (D.) of the dynamic permeability for the investigated sintered and synthetic samples, whereby standard values for water as saturation fluid is used. It can be seen, that the inertial effects appears earlier at lower frequencies with increasing frequency-dependent intrinsic permeability $k_{z}^{s}$. Figure 3.3 illustrates also that the critical frequency decreases with increasing diameter of the pore channel. The imaginary part of the dynamic permeabilities, illustrated in Figures 3.2 (D.) and 3.3 (D.), show distinct peaks at approximately the critical frequencies, which characterizes the transition from a viscous dominated flow regime to a inertial dominated flow regime. 
Table 3.2: Input parameters for the investigated artificially produced samples consisting of capillary tubes.

\begin{tabular}{lccccc}
\hline Parameter & Unit & $\mathrm{D} 1$ & $\mathrm{D} 2$ & $\mathrm{D}^{2} \mathrm{~S}^{[A]}$ & $\mathrm{D}^{[A]}$ \\
\hline \hline $\begin{array}{l}\text { Mean tube diameter } \\
d_{t, \text { mean }}\end{array}$ & {$[\mathrm{mm}]$} & 0.50 & 0.75 & 0.625 & 0.625 \\
$\begin{array}{l}\text { Maximum tube diame- } \\
\text { ter } d_{t, \text { max }}\end{array}$ & {$[\mathrm{mm}]$} & 0.50 & 0.75 & 0.75 & 0.75 \\
$\begin{array}{l}\text { Minimum tube diame- } \\
\text { ter } d_{t, \text { min }}\end{array}$ & {$[\mathrm{mm}]$} & 0.50 & 0.75 & 0.50 & 0.50 \\
$\begin{array}{l}\text { Intr. permeability } k_{z}^{s} \\
\text { Effect. porosity }\end{array}{ }^{[B]} \phi_{0}$ & {$\left[\mathrm{~m}^{2}\right]$} & $7.8125 \mathrm{E}-13$ & $3.9551 \mathrm{E}-12$ & $1.3048 \mathrm{E}-12$ & $4.7363 \mathrm{E}-12$ \\
Shape factor $M$ & $1.00 \mathrm{E}-2$ & $2.55 \mathrm{E}-2$ & $1.2625 \mathrm{E}-2$ & $3.25 \mathrm{E}-2$ \\
Tortuosity ${ }^{[C]} \alpha$ & {$[-]$} & 1 & 1 & 0.9715 & 1 \\
Crit. frequency ${ }^{[C]} f_{c}$ & {$[-]$} & 1 & 1 & 1.1736 & 1 \\
\hline
\end{tabular}

$\overline{[A]}$ : Series connection of two capillary tubes with different radii of 0.5 and $0.75 \mathrm{~mm}$.

${ }^{[B]}$ : Parallel connection of two capillary tubes with different radii of 0.5 and $0.75 \mathrm{~mm}$.

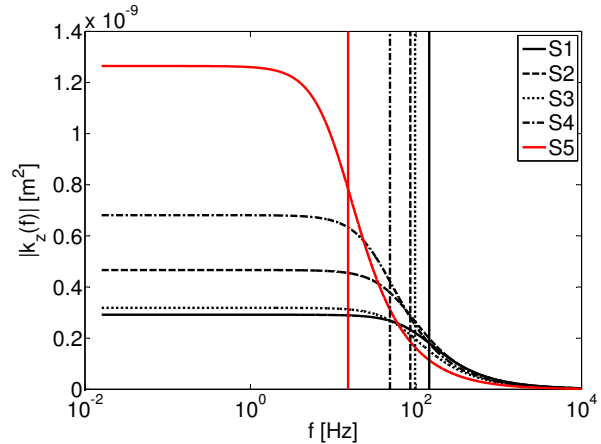

A.)

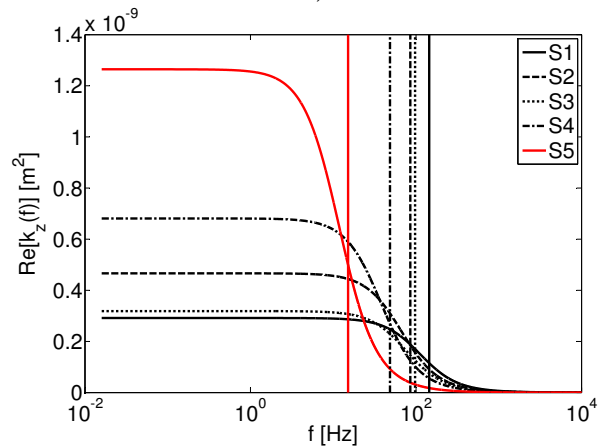

C.)

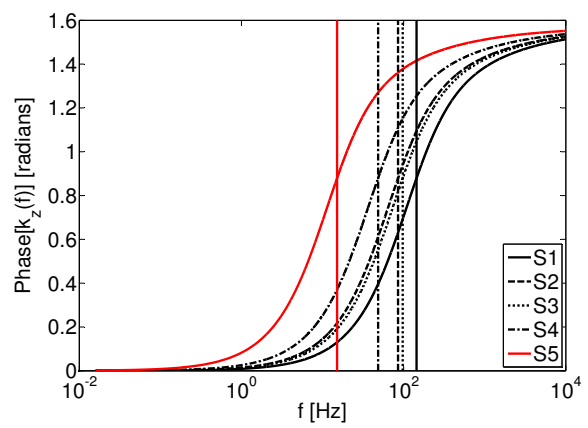

B.)

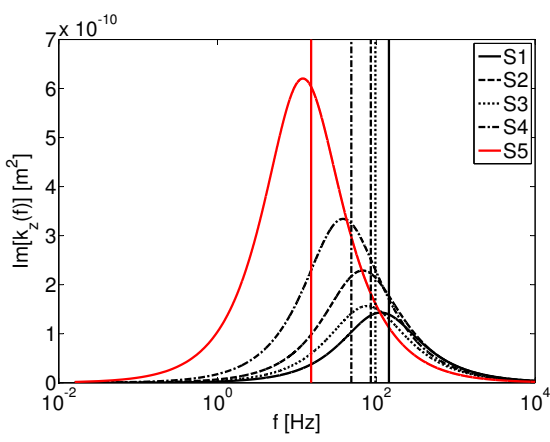

D.)

Figure 3.2: Theoretical prediction of the dynamic permeabilities of the investigated sintered samples: A.) Absolute value B.) Phase C.) Real part D.) Imaginary part. 
Charlaix et al. [44] and Sheng et al. [164] have experimentally investigated the dynamic permeability in fused and crushed glass beads and sedimentary rocks and could confirm the prediction of the dynamic permeability according to the JKD-model. Smeulders et al. [166] and Schoemaker et al. [160, 161] could confirm the same observations on sand grains and samples consisting of capillary tubes.

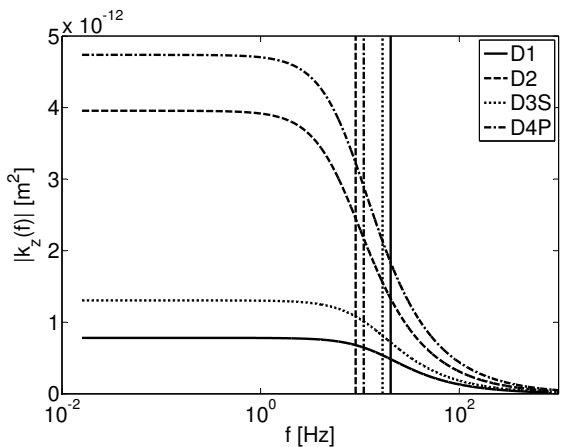

A.)

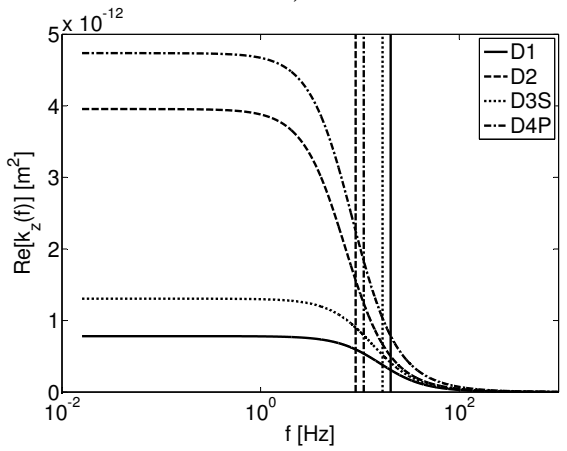

C.)

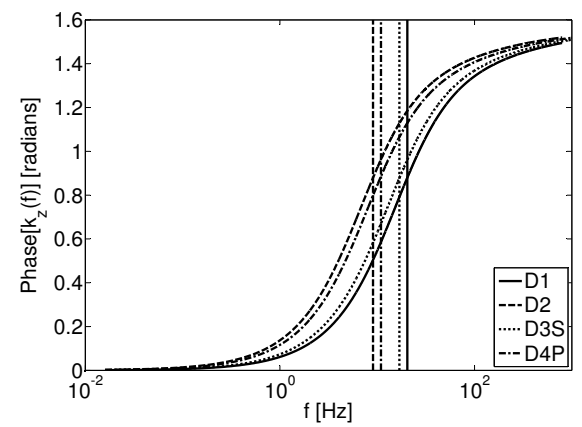

B.)

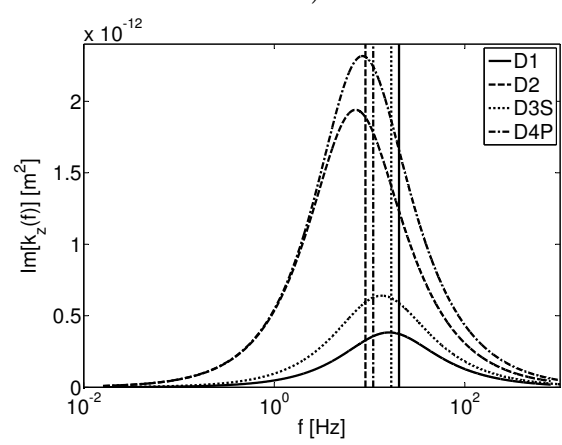

D.)

Figure 3.3: Theoretical prediction of the dynamic permeabilities of the investigated synthetic samples: A.) Absolute value B.) Phase C.) Real part D.) Imaginary part.

However, Equation (3.12) is not sufficient to be used in dynamic permeability or in so-called pore pressure oscillation experiments, since the dynamic permeability cannot be directly measured in the experiments. It is determined from the mea- 
sured time-dependent pressures of the up- and downstream reservoir. Therefore, a determination equation for the dynamic permeability is required, which takes the measured frequency-dependent pressures at the down- and upstream reservoir into account, in addition to the geometrical and initial value problem present in the experimental set-up. Furthermore, the JKD model does not provide information, which configuration of the set-up parameters, such as the water level, valve position or amplitude level for the generation of sinusoidal waves is suitable to determine the frequency-dependent hydraulical parameters of the porous medium. Therefore, the following sections deal with the derivation of adequate expressions for the dynamic permeability and further frequency-dependent hydraulical parameters such as the storage capacity or the diffusivity, which can be used in harmonic experiments by measuring the pressure signals in the up- and downstream reservoir.

\subsubsection{Derivation of determination equation for the dynamic permeability in harmonic experiments}

Schoemaker et al. [160, 161] has investigated the frequency-dependent permeability and electro-kinetic coupling coefficient in synthetic samples consisting of capillary tubes and derivated for the comparison of experimental data with the JKD-model an expression for the dynamic permeability on the basis of the wellknown Darcy's law

$$
k_{z}^{s}=\frac{\phi_{0} v_{p}}{\frac{p_{A}-p_{E}}{L_{p}} \eta^{f R}},
$$

where $L_{p}$ is the sample length and $v_{p}$ the averaged fluid velocity in the pore channels. $p_{E}$ and $p_{A}$ are the pressures at the in- and outlet of the sample. It has been attempt to formulate an expression for dynamic permeability in dependence on the measured pressures $p_{1}$ and $p_{2}$, cf. Figure 3.7 (B.). Note, that in his work $[160,161]$ the measuring cell has been filled up to a certain level, so that the distance between pressure transducer in the downstream reservoir and water surface is given by $L_{w}$, see Figure 3.7 (B.). For the derivation of the frequencydependent permeability, the fluid is assumed to be incompressible and the volume and viscous forces are neglected, so that the mass- and momentum balances within the experimental zone, illustrated in Figure 3.7 (B.) can be formulated as

$$
v_{i} A_{i}=\text { const } . \quad \text { and } \quad \rho^{f R} \ddot{u}_{f}+\operatorname{grad} p / L_{i}=0,
$$

where the index $i$ refer to the control area between two stations within the considered experimental zone and $A_{i}$ the flowed cross-sectional area. Using the harmonic approaches for fluid displacement and pressure, formulated in Equation (3.6), a frequency-dependent expression for the permeability can be formu- 
lated as

$$
k_{z}(\omega)=\frac{1}{i \omega} \frac{L_{p} \delta_{A}}{L_{w}} \frac{\eta^{f R}}{\rho^{f R}}\left(1+\frac{\delta_{A}}{L_{s}}\left(z_{1}+z_{2}\right)-\frac{\hat{P}^{u s}(z, \omega)}{\hat{P}^{d s}(z, \omega)}\right)^{-1},
$$

where $\delta_{A}=\left(A_{p}-A_{t}\right) / A_{p}$ describes the reduction of the cross-section due to the presence of the pressure transducer in the downstream reservoir. $\hat{P}^{u s}(z, \omega)$ and $\hat{P}^{d s}(z, \omega)$ refer to the complex-valued measured pressures at up- and downstream reservoir. Note, that for the derivation of the frequency-dependent permeability, the fluid flow is assumed to be one-dimensional $\left(\mathbf{v}=v_{i} \mathbf{e}_{z}\right)$ and that the fluid pressure at the water surface is completely decayed $\left(p_{3}=0\right)$. In accordance with [161] the mass and momentum balances were only considered in flow direction $z$. For a detailed derivation of Equation (3.23), we refer to [161]. For the determination of the convergence behavior of the Equation (3.23) for $\omega \rightarrow 0$ and $\omega \rightarrow \infty$, it should be taken into account that the measured pressure $\hat{P}^{u s}(z, \omega)$ and $\hat{P}^{d s}(z, \omega)$ are also highly frequency-dependent.

Equation (3.23) has been tested for different set-up configurations, such as the valve position in the downstream reservoir and different water levels. Figure 3.4 shows exemplary the measured dynamic permeability for the sintered sample S1, whereby different parameters are tested during the experiments. In the first measurement series, represented by red points, the measuring cell is filled completely with water and the valve of the downstream reservoir is closed, whereas in the second measurement series, the measuring cell is filled up to certain water level and the valve of the downstream reservoir is opened. It can be seen in Figure 3.4 that in both cases the dynamic permeability of the sintered sample decreases due to inertial effects. It is worth mentioning that the absolute of the dynamic permeabilities at lower frequencies $(f<1 \mathrm{~Hz})$ are found to be significantly greater (by factor of $10^{4}$ ) than the measured stationary permeability values.

Moreover, it is found out that the signal to noise ratio of the received pressure signals considerably increases, when the cell is completely filled with distilled water and closed. Therefore, excitation signals with smaller amplitudes can be used to generate monochromatic waves and non-linearity effects in the pressure field can be avoided. Moreover, the system remains stable (no fluctuation of the water level) especially at very low and high frequencies due to excitation of the membrane with smaller amplitudes. Therefore, the next section deals with pore pressure oscillation tests, whereby the measuring cell including the porous sample is filled completely with distilled water without any air pockets and closed towards the outside. 


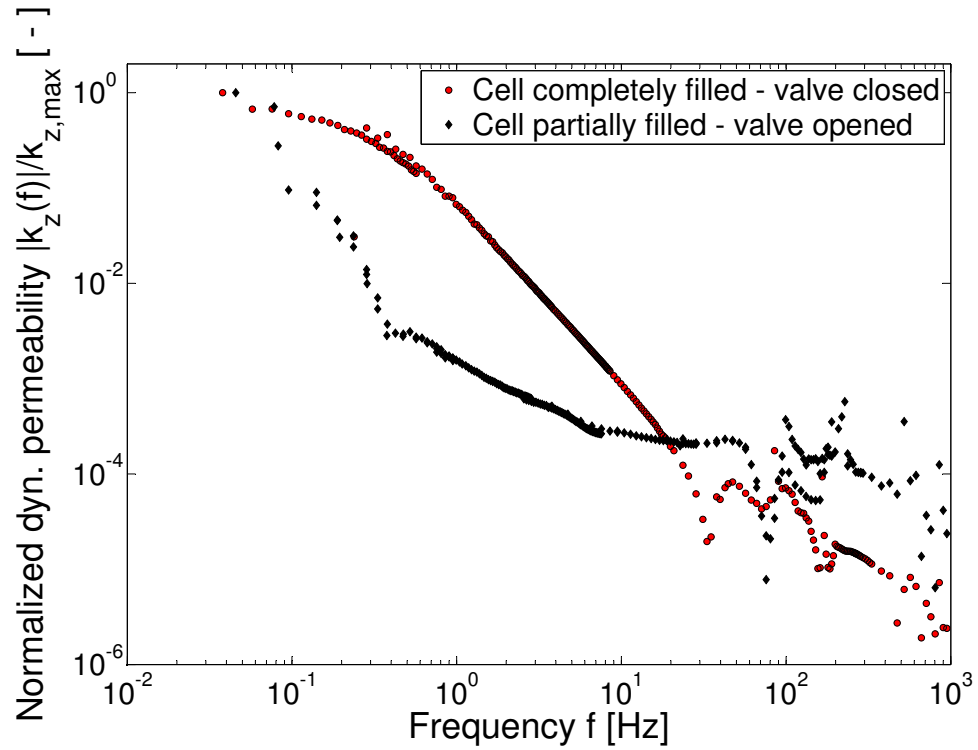

Figure 3.4: Normalized measured dynamic permeabilities for the sintered sample $\mathrm{S} 1$ at different experimental configurations. 


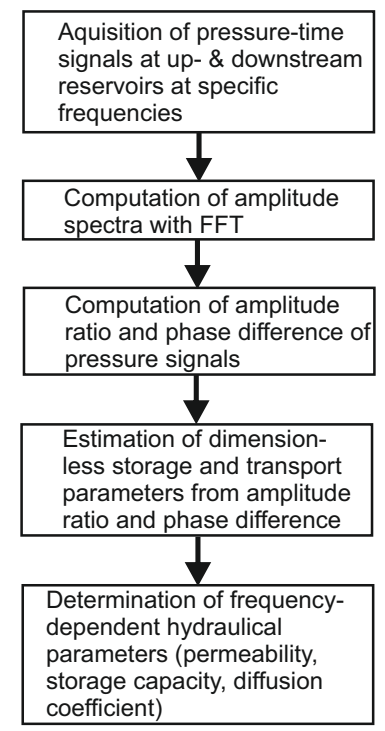

Figure 3.5: Flow chart for the determination of frequency-dependent hydraulical parameters from pore pressure oscillation method.

\subsubsection{Pressure pore oscillation method and case study}

In the so-called pore pressure oscillation method, which are usually applied for low-permeable rocks, where the flow rates are to small to be measured and controlled [19, 20, 102], the amplitude ratio and the phase difference between the upstream and downstream reservoir pressures is used to calculate both the permeability and diffusivity. In the following sections, we describe and discuss the mathematical framework of the pore pressure oscillation tests. Moreover, the corresponding equations are derived and discussed for different cases (static or dynamic case, time or frequency domain). Figure 3.5 illustrates the basic procedure for the determination of the frequency-dependent hydraulical parameters from the so-called pore pressure oscillation method by means of a flow chart. By considering the balance of mass of the pore fluid (with $\rho^{f R}=$ const.)

$$
\partial_{t}\left(\rho^{f R}\right)+\rho_{0}^{f R} \operatorname{div} \mathbf{w}_{f}=0
$$

and assuming a linear barotropic fluid $\left(p\left(\rho^{f R}\right)\right.$, i.e. $\left.p \propto \rho^{f R}\right)$ with

$$
\partial_{t}\left(\rho^{f R}\right)=\frac{\rho_{0}^{f R}}{K^{f}} \partial_{t}(p) \quad \text { and } \quad \rho^{f R}=\rho_{0}^{f R}+\frac{\rho_{0}^{f R}}{K^{f}} p
$$


we obtain a pressure velocity formulation for the balance of mass

$$
\partial_{t}(p)+K^{f} \operatorname{div} \mathbf{w}_{f}=0 .
$$

\section{D quasi static case - time domain}

Under 1-dim. quasi-static state and creeping flow in direction $z$, where inertial effects $\phi_{0} \rho_{0}^{f R} \dot{\mathbf{w}}_{f}$ and the frequency-dependent correction function $F(\omega)$ can be neglected, we obtain the well-known diffusion equation by inserting Equation (3.5) in Equation (3.26)

$$
\partial_{t}(p)-D \partial_{z z}(p)=0,
$$

whereby D represents the real-valued diffusion coefficient and can be related to hydraulic and rock parameters as followed

$$
D=\frac{k_{z}^{s} K^{f}}{\phi_{0} \eta^{f R}}=\frac{k_{z}^{s}}{\eta^{f R} s_{c}}=\frac{\pi L_{p}^{2} \eta f}{\zeta},
$$

As can be seen from Equation (3.28), the diffusion coefficient quantifies the ratio between the transport represented by $k_{z}^{s}$ and storage capacity represented by $s_{c}$ of the porous medium [169]. Besides hydraulical problems, the diffusion coefficient is a characteristic parameter in acoustical wave propagation, which highly influences the propagation of the so-called slow Biot wave [24, 25, 27, 104, 147, 169]. In the low-frequency regime, when the diffusion coefficient is high, the slow wave becomes a diffusive character cannot propagate, whereas in the high-frequency regime the diffusion coefficient and the intrinsic permeability decreases, and the relative motion between the solid and fluid required for slow wave propagation is not more impeded by viscous drags [87].

In accordance with Kranz et al. [102], we formulated the boundary conditions at the upstream and downstream reservoir for the pressure oscillation experiment as followed

$$
\begin{array}{r}
p(z, t)=P(z, \omega) \exp (i \omega t), \quad \forall z \text { on } \Gamma_{D}^{u s} \text { and } \\
\partial_{t}(p) \quad+\left(\phi_{0} D \frac{A}{V^{d s}}\right) \partial_{z}(p)=0, \quad \forall z \text { on } \Gamma_{R}^{d s} .
\end{array}
$$

In Equation (3.29) the Dirichlet boundary condition at the upstream reservoir and in Equation (3.30), a so-called Robin boundary condition at the downstream reservoir are introduced. The sample cross section is given by $A$, while the volume of the downstream reservoir is represented by $V^{d s}$. For further physical interpretation, the Robin BC in the downstream reservoir in Equation (3.30) can 
be written in a pressure-volume flux formulation as follows

$$
\partial_{t}(p)+\left(\phi_{0} K^{f} \frac{A}{V^{d s}}\right) w_{f}=0, \quad \forall z \text { on } \Gamma_{R}^{d s}
$$

Equation (3.31) illustrates clearly the effect of the downstream volume on the accuracy of permeability determination from the pressure oscillation method. If a volumetrically fuid flux with $Q=w_{f} A$ is entering the downstream reservoir in the time period $\Delta t$, a change of the fluid volume $\left(\Delta V^{d s}=Q \Delta t\right)$ and subsequently a change of the pressure in the downstream reservoir is observed according to the state of the fluid described in Equation (3.25):

$$
\frac{\Delta V^{d s}}{V^{d s}} K^{f}=\Delta p
$$

Equation (3.32) clearly demonstrates that a high pressure signal is observed in the downstream reservoir, if the downstream volume $V^{d s}$ is low and the bulk modulus of the pore fluid $K^{f}$ is high. An increase of the downstream reservoir results in a decrease of the signal-to-noise ratio of the pressure in the downstream reservoir. From the experimental point of view, the signal-to-noise ratio in the downstream reservoir becomes important especially at high frequencies when the energy dissipation of the wave propagated through the porous sample is very high.

Equation (3.26) is analytically solved using the boundary conditions formulated in Equations (3.29) and (3.30) by several authors in the past [102, 169]. For a detailed derivation of the time domain solution of the 1-dim static case, we refer to $[19,102,169]$.

Kranz et al. [102] has first introduced dimensionless parameters, in order to determine the permeability and diffusivity from the measured amplitude ratios and phase difference of the measured pressure signals. In this respect, he has also proposed a graphical map to determine the permeability and diffusivity. According to [169] the steady-state amplitude ratios and phase difference between the pressure signals in the up- and downstream reservoir reads

$$
\begin{aligned}
A_{r} & =\left[\mid \cosh \left[(1+i) \sqrt{\frac{\zeta}{\eta}}\right]+\frac{1+i}{\sqrt{\zeta \eta}} \sinh \left[(1+i) \sqrt{\frac{\zeta}{\eta}}\right]\right]^{-1} \\
\Delta \theta & =\arg \left[\left[\cosh \left[(1+i) \sqrt{\frac{\zeta}{\eta}}\right]+\frac{1+i}{\sqrt{\zeta \eta}} \sinh \left[(1+i) \sqrt{\frac{\zeta}{\eta}}\right]\right]^{-1}\right]
\end{aligned}
$$


where the dimensionless storage and transport parameters are given by

$$
\zeta=\frac{A L_{p}}{s_{c}^{d s}} s_{c} \quad \text { and } \quad \eta=\frac{A}{\pi \eta^{f R} L_{p} s_{c}^{d s} f} k_{z}^{s}
$$

Here, $f$ and $L_{p}$ represent the oscillation frequency and sample length. $s_{c}^{d s}$ is the storage capacity of the downstream reservoir, which can be expressed as followed:

$$
s_{c}^{d s}=\frac{V^{d s}}{K^{f}} .
$$

For the used experimental set up the storage capacity of the downstream reservoir is determined at $s_{c}^{d s}=6.25 \times 10^{-14} \mathrm{~m}^{3} / \mathrm{Pa}$ with $\kappa^{f}=1 / K^{f}=4.58 \times 10^{-10} \mathrm{~Pa}^{-1}$ as the compressibility of water.

\section{1-dim dynamic case - time domain}

In the dynamic case we are not able to reduce the hydro-mechanical problem to a single diffusion equation as discussed in the previous section. The problem is coupled in the variables $P=\left\{w_{f}, p\right\}$ and reads

$$
\begin{aligned}
\partial_{t}\left(w_{f}\right)+\frac{K^{f}}{D \rho_{0}^{f R}} w_{f}+\frac{1}{\rho_{0}^{f R}} \partial_{z}(p) & =0 \\
\partial_{t}(p)+K^{f} \partial_{z}\left(w_{f}\right) & =0 .
\end{aligned}
$$

The solution of Equations in (3.37) can only be obtained by solving the coupled set of partial differential equations. The boundary conditions are the ones already formulated in Equations (3.29) and (3.30).

\section{1-dim quasi-static case - frequency domain}

The diffusion equation in (3.27) is transformed to the frequency space by applying the harmonic ansatz for fluid pressure $p$ and seepage velocity $w_{f}$ in Equation (3.6)

$$
\hat{p}+\hat{D} \partial_{z z}(\hat{p})=0 \quad \text { with } \quad \hat{D}=\frac{i k_{z}^{s} K^{f}}{\omega \phi_{0} \eta^{f R}}
$$

Equation (3.38) is Helmholtz equation with a complex-valued diffusion coefficient whose real part is zero. It should be noticed that Darcy's law can be formulated w.r.t the frequency-transformed quantities

$$
\hat{w}_{f}=\frac{1}{\eta^{f R} \phi_{0}} k_{z, 0}^{s} \partial_{z}(\hat{p}) .
$$


The boundary conditions of the Helmholtz equation in (3.38) are given by

$$
\begin{array}{r}
p=\overline{\hat{p}} \quad \forall z \text { on } \Gamma_{D}^{u s} \text { and } \\
i \omega \hat{p}+\left(\phi_{0} D \frac{A}{V^{d s}}\right) \partial_{z}(\hat{p})=0, \quad \forall z \text { on } \Gamma_{R}^{d s} .
\end{array}
$$

\section{1-dim dynamic case - frequency domain}

For the derivation of the frequency-dependent dynamic equation for the permeability the balance of momentum in Equation (3.5) is again transformed to the frequency domain using the harmonic approach for fluid pressure and velocity in Equation (3.6)

$$
i \omega \rho_{0}^{f R} \hat{w}_{f}-\frac{\eta^{f R} \phi_{0}}{k_{z, 0}^{s}} \hat{w}_{f}+\partial_{z}(\hat{p})=0
$$

Equation (3.42) can be reformulated as a dynamic Darcy equation, which only depends on the introduced pressure amplitudes and seepage velocity

$$
\hat{w}_{f}=\frac{1}{\eta^{f R} \phi_{0}}\left[\frac{\eta^{f R} \phi_{0} k_{z, 0}^{s}}{\eta^{f R} \phi_{0}-i \omega \rho_{0}^{f R} k_{z, 0}^{s}}\right] \partial_{z}(\hat{p}):=\frac{1}{\eta^{f R} \phi_{0}}\left[k_{z}(\omega)\right] \partial_{z}(\hat{p}) .
$$

In contrast to Equation (3.39), the coefficient in Equation (3.43) is frequencydependent and complex-valued. Inserting Equations (3.43) into the frequencytransformed mass balance from Equation (3.37) yields

$$
i \omega \hat{p}+K^{f} \partial_{z} \hat{w}_{f}=0 \quad \text { or } \quad \hat{p}-i \frac{K^{f}}{\omega} \partial_{z} \hat{w}_{f}=0 .
$$

Inserting Equation (3.43) in (3.44) finally yields

$$
\hat{p}+i \frac{K^{f} k_{z}^{s}(\omega)}{\omega \eta^{f R} \phi_{0}} \partial_{z z} \hat{p}=0 .
$$

Multiplication Equation (3.45) with the complex conjugate yields the following expression for the for the dynamic and frequency-dependent permeability introduced in Equation (3.43)

$$
k_{z}^{s}(\omega)=\underbrace{\frac{\left(\eta^{f R} \phi_{0}\right)^{2} k_{z, 0}^{s}}{\left(\eta^{f R} \phi_{0}\right)^{2}\left(\omega \rho_{0}^{f R} k_{z, 0}^{s}\right)^{2}}}_{\operatorname{Re}\left[k_{z}^{s}(\omega)\right]}+i \underbrace{\frac{\left.\omega \eta^{f R} \phi_{0} \rho^{f R}\left(k_{z, 0}^{s}\right)^{2}\right)}{\left(\eta^{f R} \phi_{0}\right)^{2}+\left(\omega \rho_{0}^{f R} k_{z, 0}^{s}\right)^{2}}}_{\operatorname{Im}\left[k_{z}^{s}(\omega)\right]} .
$$


Introducing the frequency-dependent dimensionless permeability and dimensionless frequency as

$$
\bar{k}_{z}^{s}(\bar{\omega})=\frac{k_{z}^{s}(\omega)}{k_{z, 0}^{s}} \quad \text { and } \quad \bar{\omega}=\frac{\omega}{\omega_{\text {crit }}}
$$

and inserting the dynamic permeability expression of Equation (3.46) into (3.47) yields

$$
\bar{k}_{z}^{s}(\bar{\omega})=\frac{k_{z}^{s}}{k_{z, 0}^{s}}=\frac{1}{1+\bar{\omega}^{2}}+i \frac{\bar{\omega}}{1+\bar{\omega}^{2}}
$$

with

$$
\operatorname{Re}\left[\bar{k}_{z}^{s}\right]=\frac{1}{1+\bar{\omega}^{2}} \quad \text { and } \quad \operatorname{Im}\left[\bar{k}_{z}^{s}\right]=\frac{\bar{\omega}}{1+\bar{\omega}^{2}}
$$

and

$$
\tan (\theta)=\frac{1}{Q}=\frac{\operatorname{Im}\left[\bar{k}_{z}^{s}\right]}{\operatorname{Re}\left[\bar{k}_{z}^{s}\right]}=\bar{\omega} \quad \text { and } \quad\left|\bar{k}_{z}^{s}\right|=\frac{1}{1+\bar{\omega}^{2}} \sqrt{1+\bar{\omega}^{2}}
$$

whereby $1 / Q$ and $\theta$ represents the specific attenuation and phase difference of the dynamic permeability. Note that the dimensionless quantities $\bar{k}_{z}^{s}$ and $\bar{\omega}$ are linked to physical quantities through characteristic quantities, like the real-valued intrinsic permeability $k_{z}^{s}$ and the critical frequency $\omega_{\text {crit }}$ shown in Equation (3.10). In accordance with the well-known JKD model the following limits result for the dimensionless permeability in the frequency domain

$$
\begin{array}{rlrl}
\lim _{\omega \rightarrow 0} \operatorname{Re}\left[\bar{k}_{z}^{s}\right]=1 & \text { and } & \lim _{\omega \rightarrow \infty} \operatorname{Re}\left[\bar{k}_{z}^{s}\right]=0, \\
\lim _{\omega \rightarrow 0} \operatorname{Im}\left[\bar{k}_{z}^{s}\right]=0 & \text { and } \quad & \lim _{\omega \rightarrow \infty} \operatorname{Im}\left[\bar{k}_{z}^{s}\right]=1, \\
\lim _{\omega \rightarrow 0} \tan (\theta) & =0 & \text { and } & \lim _{\omega \rightarrow \infty} \tan (\theta)=\infty, \\
\lim _{\omega \rightarrow 0}\left|\bar{k}_{z}^{s}\right|=1 & \text { and } & \lim _{\omega \rightarrow \infty}\left|\bar{k}_{z}^{s}\right|=0 .
\end{array}
$$

\subsection{Experiments}

In this section the experimental set-up is presented and the signal processing of the received pressure-time-signals for the determination of the amplitude ratio and phase differences is described in detail. Moreover, the frequency-dependent hydraulical parameters are determined for specific samples and compared with stationary permeabilities obtained from classical Darcy experiments. 


\subsubsection{Experimental rig}

As can be seen in Figure 3.6 D.), the dynamic Darcy cell is transparent and fixed on a specially developed table. Prior to the harmonic experiments, the dynamic Darcy cell including the porous sample is evacuated and subsequently filled with carbon dioxide. In the next step the cell is completely flooded with distilled water without any air pockets. The carbon dioxide easily dissolves in the water and an optimal saturation of the sample is guaranteed.

An oscillatory fluid flow is induced by means of an shaker (Vibration exciter Brüel \& Kjaer, model 4809), which drives an piston rod, cf. Figure 3.6 (C., D.). The oscillatory movement of the rod is transferred to a latex membrane equipped with plate made of plastic, which finally generates an oscillatory fluid flow in the measuring cell, cf. Figure 3.6 (A., D.). In this manner continuous monochromatic sinusoidal waves could be generated. The height of the piston rod is chosen so that the membrane is only subjected to pressure at any time and position of the piston rod.

The shaker is driven by an arbitrary function generator (Agilent, 33500B series, $30 \mathrm{MHz}$-waveform generator), which is connected to a power amplifier (Brüel \& Kjaer, model 2706 or 2718), see Figure 3.7 (A). The pressure drop across the porous sample is measured by using piezo-electric transient pressure transducers (PCB 112A22). The first transducer is installed in the lower chamber laterally just before the porous sample, whereas the second transducer is located in the center field of the upper chamber. The received pressure signals are amplified by using a measuring amplifier (HBM, model QuantumX MX410) and finally displayed and stored on a computer.

\subsubsection{Time signals and amplitude spectra}

For the excitation of the latex membrane, continuous sinus signal with different frequencies ranging from $0.1 \mathrm{~Hz}$ to $1000 \mathrm{~Hz}$ are used. Depending on the frequency of the wave and the signal to noise ratio of the received pressure signals, the amplitude of the generated wave ranged between 1 and $6 \mathrm{~V}$. It is important that the oscillation amplitude is small and chosen to be only a small fraction of the ambient pore pressure to avoid poroelastic and fluid compressibility changes. 


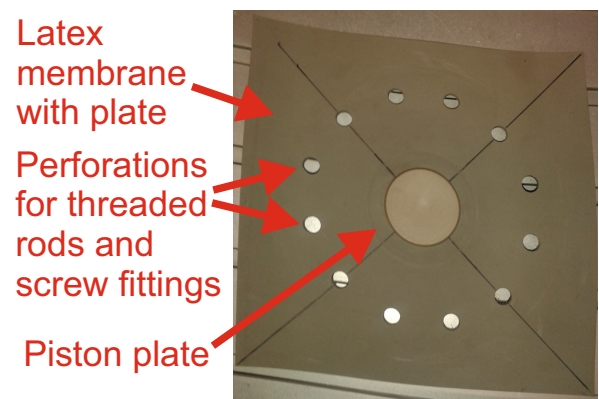

A.)

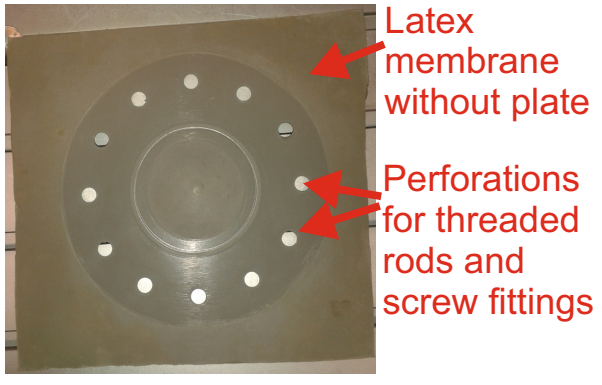

B.)

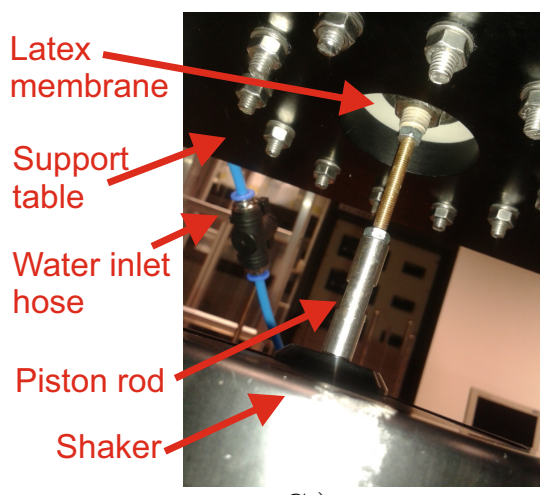

C.)

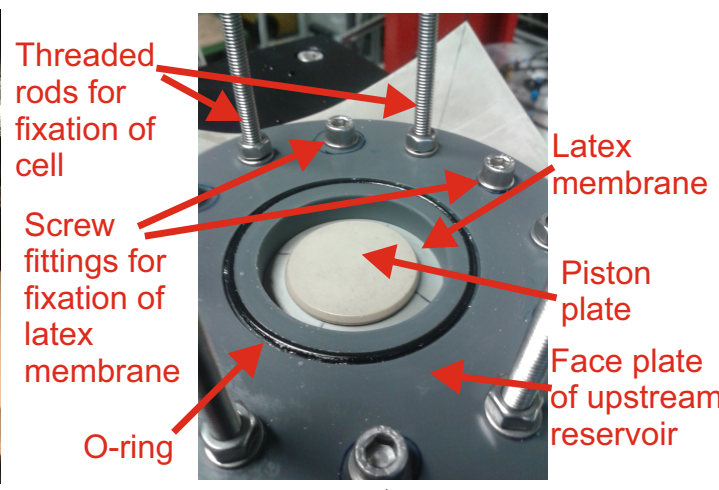

D.)

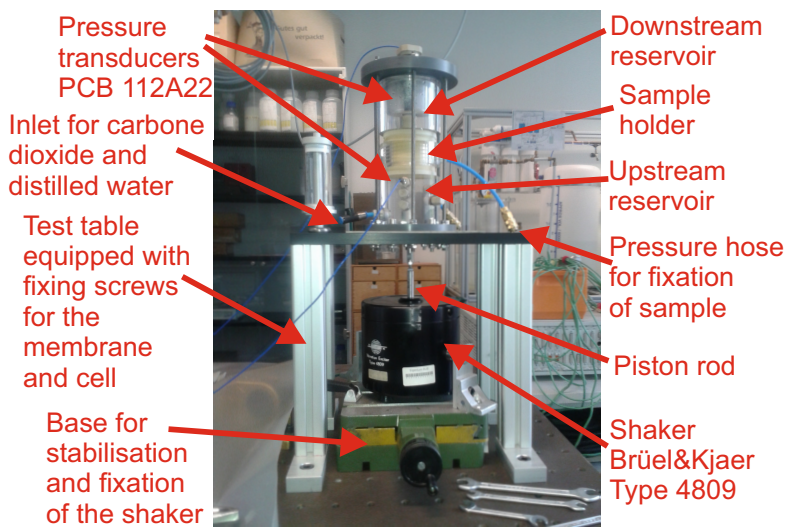

E.)

Figure 3.6: Photographs of latex membrane without (A.) and with (B.) a plastic plate, which serves to increase the stiffness of the latex membrane. Photographs from the bottom view of the test table and of the mounted latex membrane inclusive front plate of the upstream reservoir are shown in C.) and D.). The Dynamic Darcy Cell (DDC) with shaker, generating monochromatic waves up to $5 \mathrm{kHz}$ is illustrated in E.). 


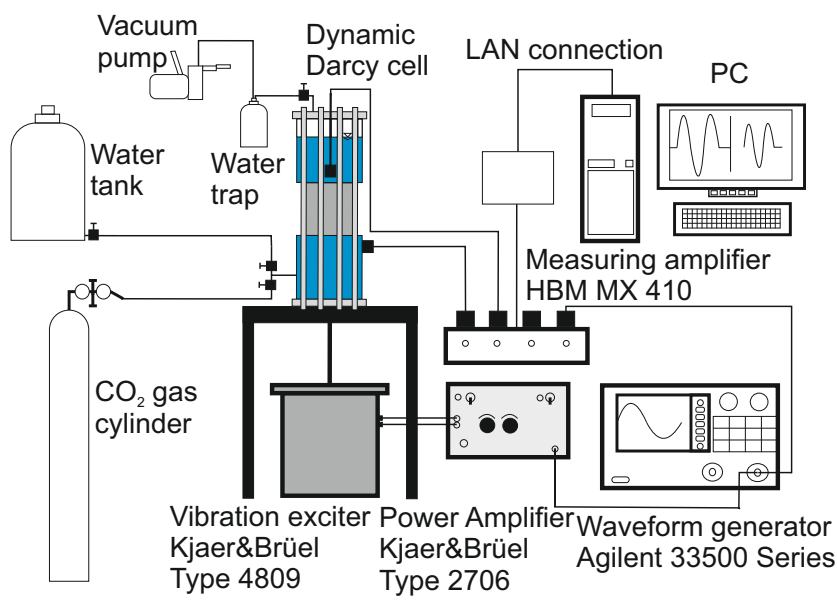

A.)

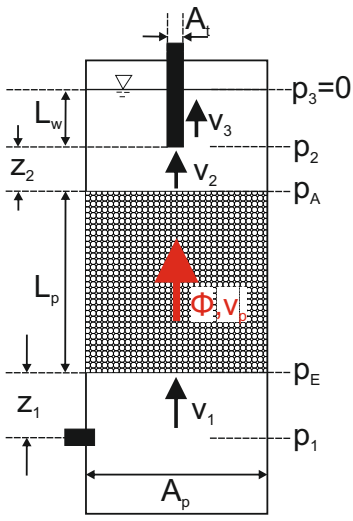

B.)

Figure 3.7: Experimental arrangement for dynamic permeability measurements (A.) and sketch of measuring cell for derivation of determination equation of dynamic permeability at partial saturation of measuring cell (B.).

As can be seen, in pressure-time-signals of the Figures 3.8, 3.9, 3.10, 3.11, $3.12,3.20,3.21,3.22,3.23$, the received time signals of the pressure transducers consists of three components [55]:

1. the true useful AC component from the forced sinusoidal cycling of the latex membrane

2. true pressure signal from temperature effects during cycling

3. electrical noise (DC component) caused by temperature effects on the electronic components, background noise etc.

It should be noticed that the water temperature has changed slightly around $0.5{ }^{\circ} \mathrm{C}$, so that the pressure component caused by temperature effects are assessed to be negligible small. Since our investigation concerns the determination of the dynamic behavior of the hydraulic properties of the porous samples, the DC component of the received pressure signals are set to zero before calculating the amplitude spectra of the pressure time signals. However, it should be noticed that the amplitude ratios show only marginal changes in the order of $10^{-4}$, while the phase differences remained unaffected, when the DC components of the received pressure signals are set to zero, because the pressure offsets (DC 
component) showed magnitudes much smaller than the alternating pressure components (AC component).

Because the signal of interest lies at a specific frequency use is made of the Discrete Fast Fourier Transformation (DFFT) as a tool for the determination of the amplitude ratios and phase differences between the pressure signals located at the up- and downstream reservoirs. Depending on the frequency of the generated wave, different time windows for the pressure signals are recorded, in order to obtain sufficient cycles during each measurement. A time window of 40 seconds is chosen for low-frequency waves $(f \leq 0.5 \mathrm{~Hz})$, whereby for higher frequency waves $(f>0.5 \mathrm{~Hz})$ a time window of 20 seconds is sufficient to record an adequate number of cycles. In contrast to low-permeable tight rock samples, the steady-state conditions of the pressure history is reached within few milliseconds. The whole time range of the received pressure signals are windowed before the computation of the FFT by using a so-called "tukey" window.

Figure 3.8 and 3.9 show exemplary the recorded pressure time signals and the corresponding amplitude spectra for the water-saturated sample S1 at $0.95 \mathrm{~Hz}$, whereby different water levels and configurations of the latex membrane (without and with a piston plate) are tested. For comparison, the Figure 3.8 and 3.9 show additionally the electrical input signals, which are used for the sinusoidal cycling of the latex membrane. Figure 3.8 shows the results when the measuring cell was filled with distilled water up to a certain level and no piston plate is used for the latex membrane, cf. Figure 3.6 B.). The comparison of the amplitude spectra of the pressure signals and the electrical input signals clearly demonstrates that the amplitude spectra of the pressure signals indicate additional several distinct peaks, which occur in a distance of $f=N f_{e}$, whereby $f_{e}$ corresponds to the oscillation frequency of the latex membrane. As denoted in Figure 3.8 B.) the peaks occuring at frequencies $f=N f_{e}$ can be clearly assigned to higher mode oscillation of the latex membrane caused by non-linearity effects. The resulting pressure signals in Figure 3.8 A.) are a superposition of several harmonic waves with different frequencies. However, the non-linearity effects are locally limited within the membrane. The pressure field in the measuring cell, and thus the hydraulical properties of investigated samples, are not affected by non-linearity effects, see section subsection 3.3.5 Amplitude sweep tests. 


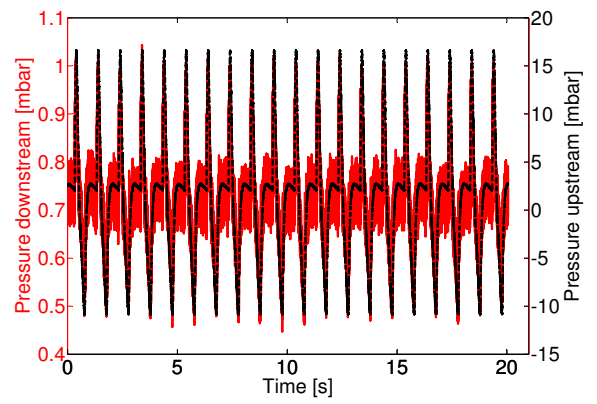

A.)

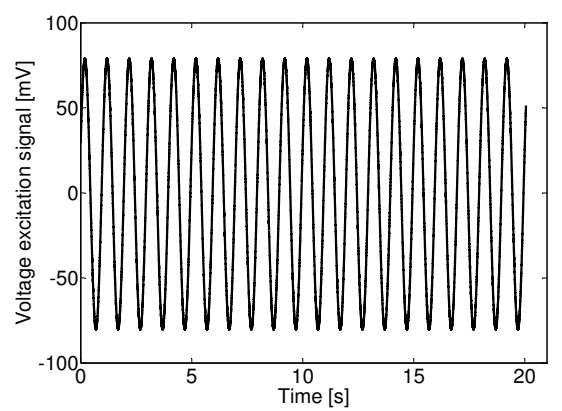

C.)

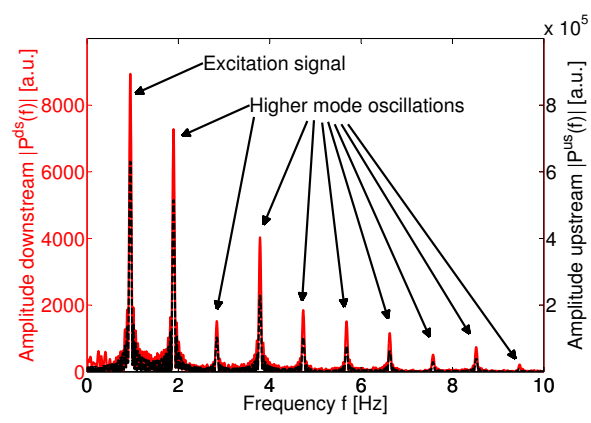

B.)

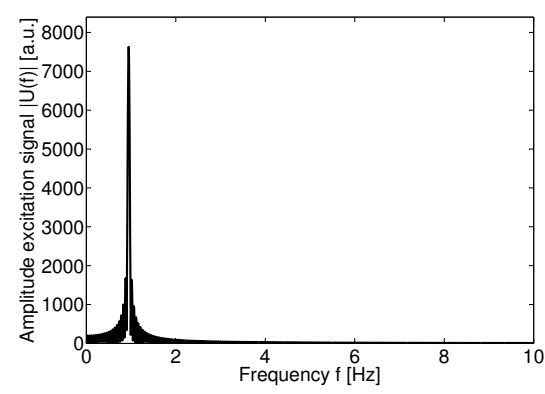

D.)

Figure 3.8: Pressure-time signals of the sintered sample $\mathrm{S} 1$ at $0.95 \mathrm{~Hz}$ obtained from piezo-resistive transducers positioned at the up- and downstream reservoir (A.) with corresponding amplitude spectra (B.). Excitation signal for the latex membrane (C.) with corresponding amplitude spectrum (D.). The measuring cell is not completely flooded with distilled water and no piston plate shown in Figure3.6 (B.) is used to increase the stiffness of the latex membrane.

In order to minimize or completely prevent the higher mode oscillations of the latex membrane, the stiffness of the membrane has been artificially increased by using a piston plate, cf. Figure 3.6 A.). As a results Figure 3.9 illustrates the time signals and amplitude spectra of the pressure and electrical input signal, when the measuring cell including the porous sample S1 is completely flooded with distilled water and a piston plate is used to generate monochromatic waves. The pressure-time signals at the down- and upstream reservoir show clean sinusoidal shapes, and the amplitude spectra exhibit only one distinct peak at the excitation frequency of $0.95 \mathrm{~Hz}$. The higher mode oscillation of the membrane 
are highly attenuated or have disappeared completely, see Figure 3.9 A.) and B.). Besides the avoiding of the higher mode oscillation of the membrane a clear signal enhancement is reached due to fully saturation of the measuring cell, especially for the pressure signal in the downstream reservoir, although the latex membrane has been oscillated with a certainly smaller amplitude. In this way, high signal-to-noise ratios even for extremely high frequencies $(f=80-1000 \mathrm{~Hz})$ could be achieved. Moreover, the resonance behavior of the experimental test rig, which especially occurred at frequencies greater than $80 \mathrm{~Hz}$ can be improved, due to smaller oscillation amplitude used for the latex membrane. The excitation of the latex membrane is not taken place point-like through the application of the piston plate, but instead the cross-sectional area is stirred uniformly.

In order to demonstrate that the pressure signals do not only depend on the excitation method or water level, but also on the frequency of the generated wave, Figure 3.10 shows the pressure and excitation signals of the sintered sample S1 at $37.87 \mathrm{~Hz}$. It is obvious that the the absolute amplitude values and the phase difference between the pressure signals have changed with increasing frequency compared to the measurement at $0.95 \mathrm{~Hz}$. The phase difference between the pressure signals leads to a noticeable time shift of the pressure signals, cf. Figure 3.10 A.).

Figures 3.11 and 3.12 illustrate the raw pressure time-signals with the corresponding amplitude spectra of further sintered sample S4 and S5 at two specific frequencies of 0.95 and $37.87 \mathrm{~Hz}$. The differences of pressure-time signals and the amplitude spectra between the investigated samples, but also between the different frequencies in terms of amplitude ratios and phase difference are clearly visible. A closer consideration of the amplitude spectra, depicted in Figures 3.10, 3.11 and 3.12, reveal the occurrence of so-called side lobes around the peak of interest. The side lobes are not of physical origin, but result from windowing and often referred as leakage effect. The windowing of sinusoidal wave forms causes the FFT to develop non zero-values around the frequency of interest. The leakage effect can be minimized by suitable choice of window functions and parameters [34].

However, the side lobes caused by leakage effect differ considerably from the main peak at the excitation frequency of interest and the peaks resulting from higher mode oscillations of the latex membrane in magnitude and shape. It should be noticed, that the different window functions and parameter have no impact on the amplitude ratios and phase differences between the pressure signals, while remaining consistent during signal data processing. 


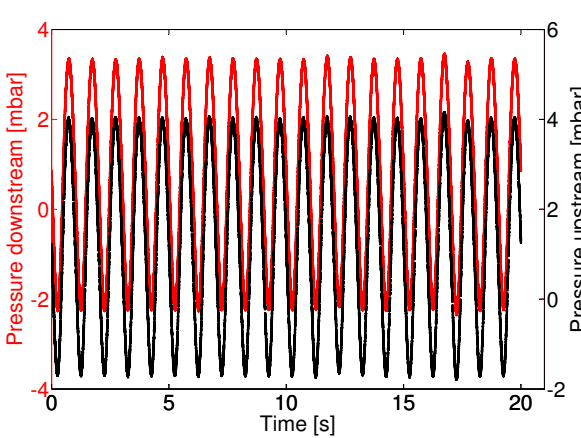

A.)

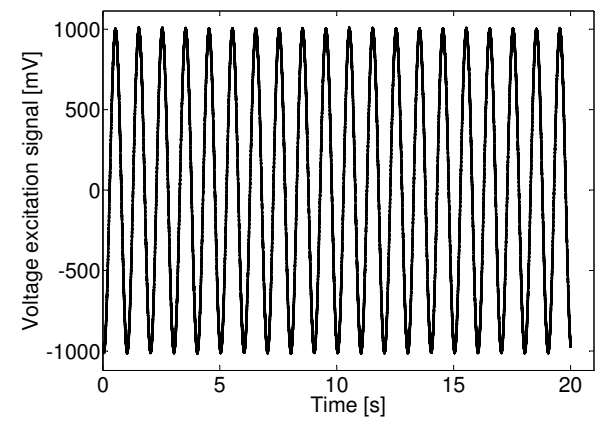

C.)

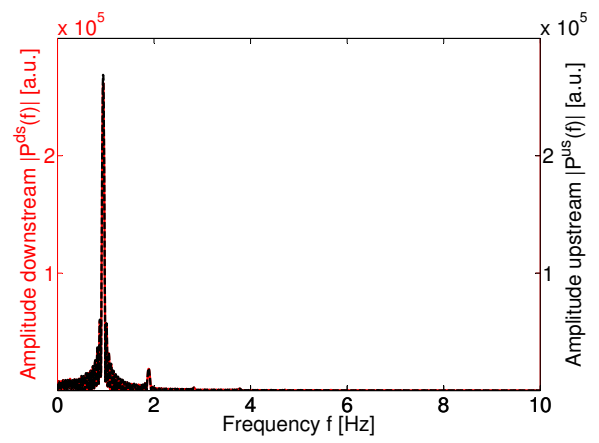

B.)

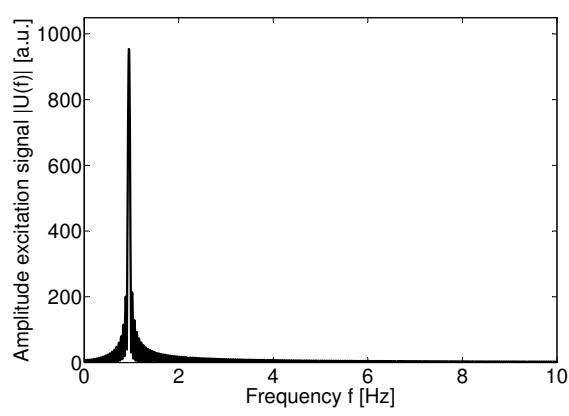

D.)

Figure 3.9: Pressure-time signals of the sintered sample S1 $0.95 \mathrm{~Hz}$ obtained from piezo-resistive transducers positioned at the up- and downstream reservoir (A.) with corresponding amplitude spectra (B.). Excitation signal for the latex membrane (C.) with corresponding amplitude spectrum (D.). The measuring cell is completely flooded with distilled water and a piston plate shown in Figure 3.6 (A.) is used to increase the stiffness of the latex membrane.

Figures 3.20, 3.21, 3.22 and 3.23 (in the appendix) illustrate the raw pressure time signals and amplitude spectra of the investigated synthetic samples, consisting of tube-like pore channels at two specific frequencies. It can be also observed that the pressure signals and amplitude spectra show a noticeable frequency and sample dependence. While the differences in the magnitude of the amplitudes between the pressure signals in the up- and downstream reservoir are very pronounced, the phase differences are very small, independently of the frequency of 


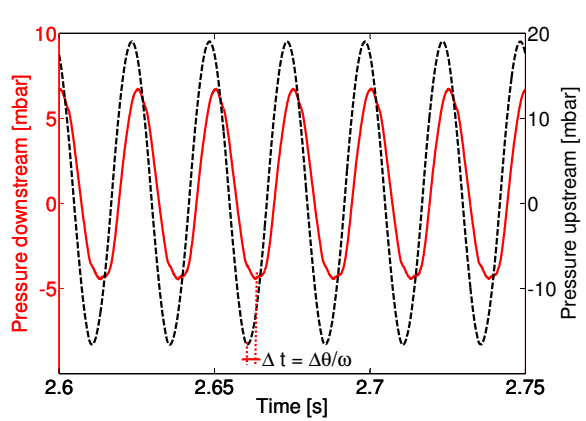

A.)

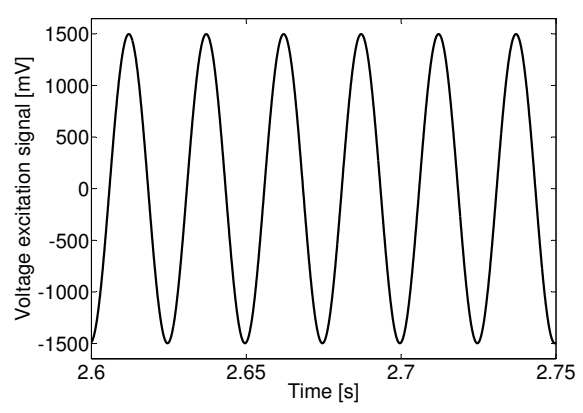

C.)

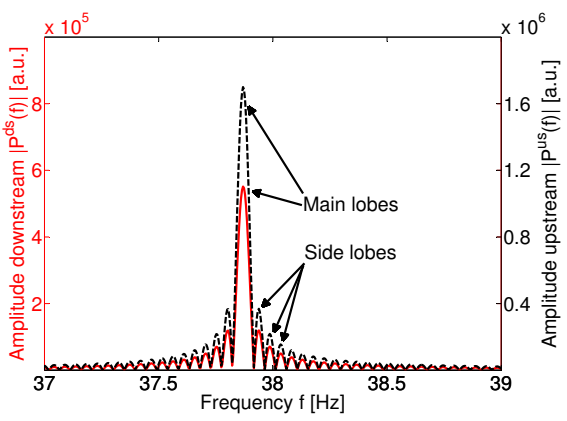

B.)

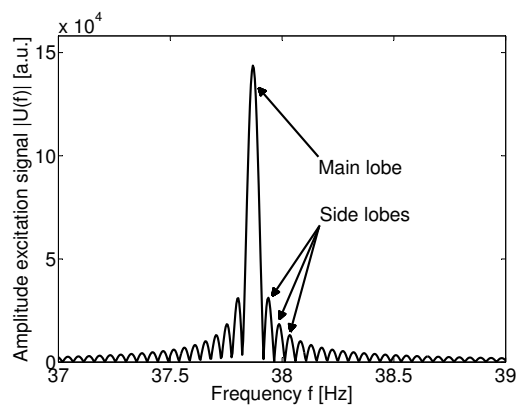

D.)

Figure 3.10: Pressure-time signals of the sintered sample S1 at $37.87 \mathrm{~Hz}$ obtained from piezo-resistive transducers positioned at the up- and downstream reservoir (A.) with corresponding amplitude spectra (B.). Excitation signal for the latex membrane (C.) with corresponding amplitude spectrum (D.). The measuring cell is completely flooded with distilled water and a piston plate shown in Figure3.6 (A.) is used to increase the stiffness of the latex membrane. 


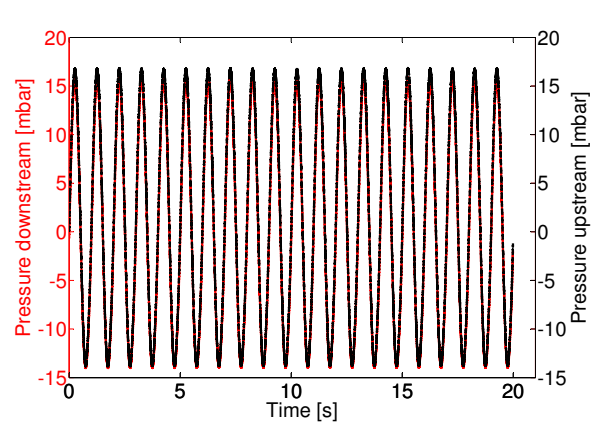

A.)

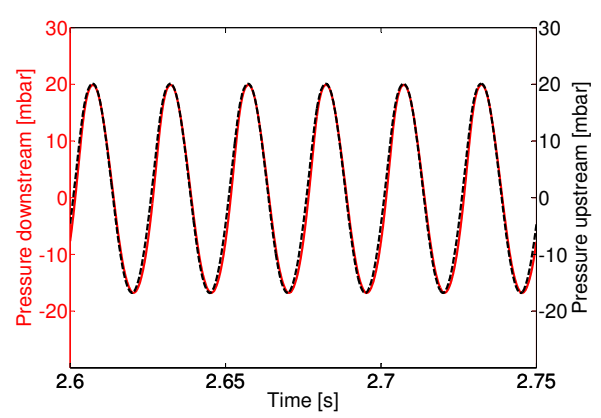

C.)

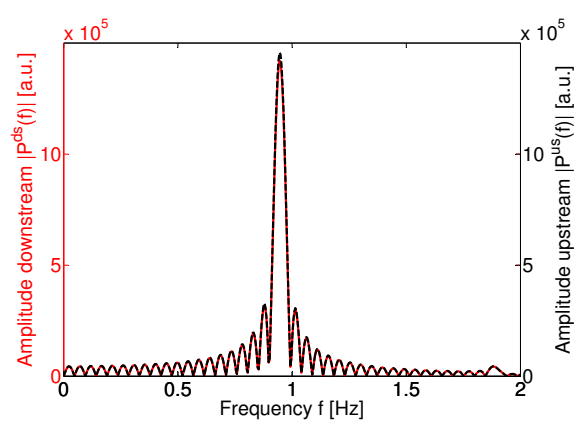

B.)

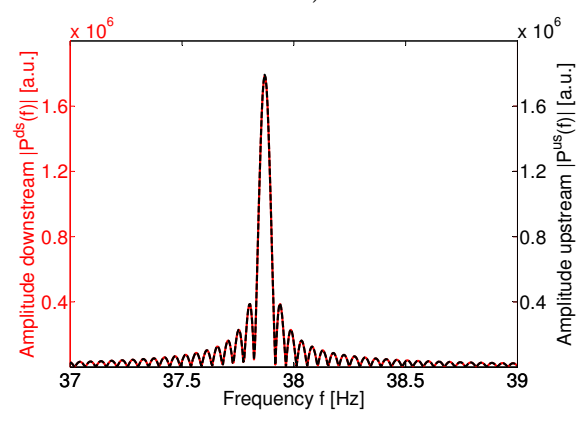

D.)

Figure 3.11: Pressure-time signals of the sintered sample S4 at 0.95 (A.) and $37.87 \mathrm{~Hz}$ (B.) with corresponding amplitude spectra (B. and D.). 


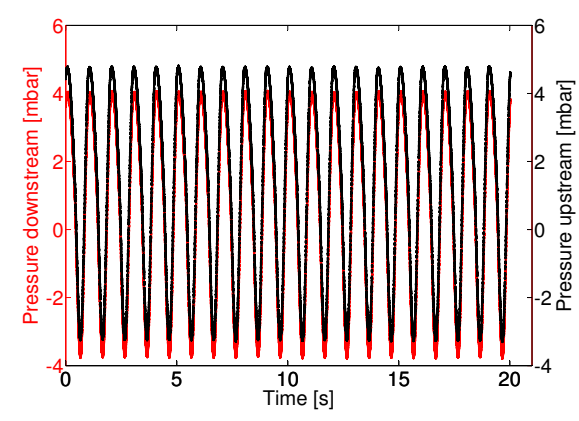

A.)

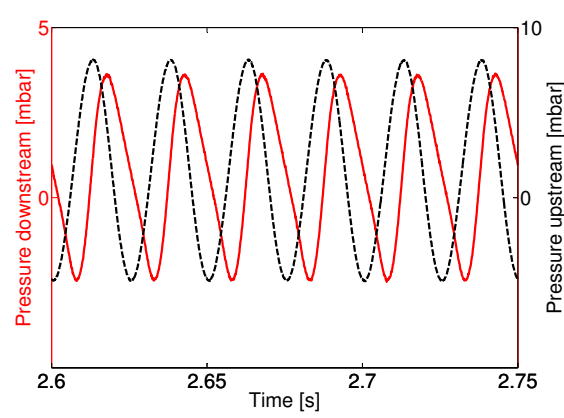

C.)

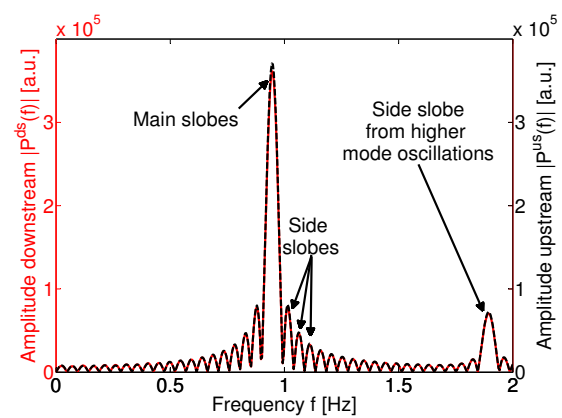

B.)

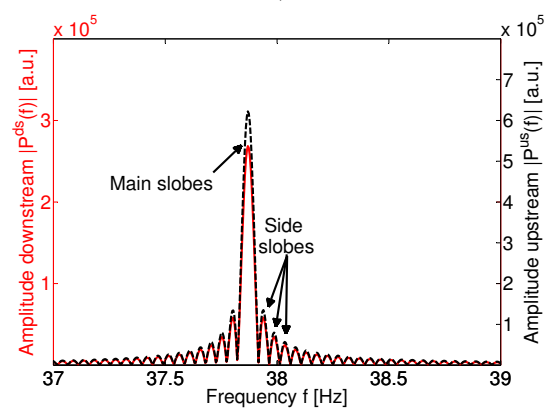

D.)

Figure 3.12: Pressure-time signals of the sintered sample S5 at 0.95 (A.) and $37.87 \mathrm{~Hz}$ (B.) with corresponding amplitude spectra (B. and D.). 
the wave. In comparison to the sintered samples, the phase differences between the signals of the synthetic samples are considerably smaller for a wide frequency range up to $100 \mathrm{~Hz}$. Moreover, it can be seen that the amplitude ratio between the down- and upstream reservoir for all synthetic samples decreases apparently with increasing frequency. 


\subsubsection{Frequency-dependent amplitude ratio and phase dif- ference}

The frequency-dependent amplitude ratio and phase difference between the pressure signals are determined from the amplitude spectrum of the signals in the frequency domain. The phase differences are computed from the cross power spectrum $G_{P 1, P 2}(f)$ of the pressure signals in the up- and downstream reservoir as follows:

$$
\left.G_{P 1, P 2}(f)=P^{d s}(f) \bar{P}^{u s}(f)\right)
$$

whereby $P^{d s}(f)$ is the complex-valued pressure amplitude of the downstream reservoir and $\bar{P}^{u s}(f)$ the complex-conjugated amplitude values of the upstream reservoir determined from FFT. The phase difference can be derived from the complex ratio of the cross power spectrum $G_{P 1, P 2}(f)$

$$
\Delta \phi(f):=\phi^{u s}(f)-\phi^{u s}(f)=\arctan \left(G_{P 1, P 2}(f)\right) .
$$

However, it should be noticed the arcus tangens function only provides values between $-\pi$ and $+\pi$, which is often referred as wrapped phase difference. For a continuous phase difference spectrum, the phase difference jumps at $2 \pi$ modulo are determined adequately by adding or subtracting multiples of $2 \pi$ to the principal values [107]. The corrected continuous phase difference is often referred as the unwrapped phase difference

$$
\Delta \phi(f)_{u}=\Delta \phi(f) \pm 2 \bar{k} \pi
$$

where $\bar{k}$ is an integer. As an example of corrected phase difference jumps, Figure 3.13 shows the wrapped and unwrapped phase difference of sample S5, where the discontinuity at approximately $f \approx 52 \mathrm{~Hz}$ is removed.

The Figure 3.14 illustrates the frequency-dependent amplitude ratios and phase difference of the investigated synthetic and sintered samples in a wide frequency range of $0.1-1000 \mathrm{~Hz}$. In all cases examined, the resonance behavior of the experimental rig becomes obvious at frequencies grater than approximately $80 \mathrm{~Hz}$, where phase difference and amplitude ratio exhibit strong fluctuations. In the resonance regime of the investigated synthetic and sintered samples, the amplitude ratios in the resonance are partially clearly greater than one, which means that the pressure in the downstream reservoir is grater than in the upstream reservoir. However, it should be noticed that the pressure transducer is locally installed peripheral just before sample for technical reasons and that it might be possible that inertial effects in the peripheral zones have additionally influenced the pressure development in the upstream reservoir, especially at frequencies above $80 \mathrm{~Hz}$. 


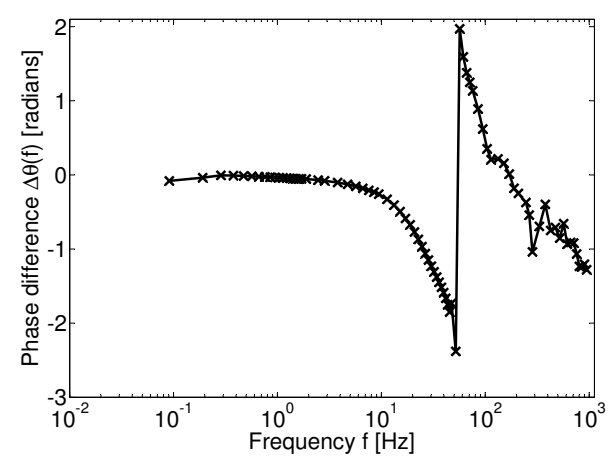

A.)

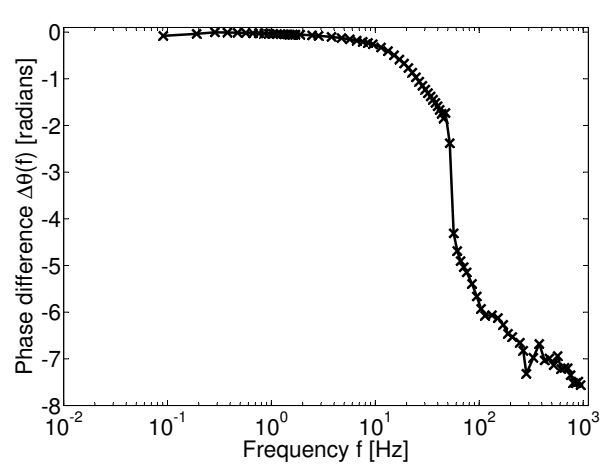

B.)

Figure 3.13: Illustration of frequency-dependent phase difference through the example of the sintered sample S5: A.) Original phase difference B.) Corrected phase difference. After unwrapping the phase jumps for frequencies greater than $52 \mathrm{~Hz}$ are eliminated.

Figure 3.15 shows the phase difference up to a frequency of approximately $100 \mathrm{~Hz}$, where resonance effects are assessed to be negligible small. It can be seen that the phase difference and amplitude ratios of the sintered samples decrease with increasing frequency up to $80 \mathrm{~Hz}$.

\subsubsection{Frequency-dependent permeabilities, diffusion coeffi- cient and storage capacity}

The frequency-dependent hydraulical parameters are determined from a grid search for the values of $\zeta$ and $\eta$ consistent with the measured amplitude ratio and phase difference of the pressure signals in the up- and downstream reservoir. Figure 3.16 shows exemplary an extract of the graphical solution of the 1-dim. diffusion equation presented in Equation (3.27) for the sintered sample S1 (A.) and the synthetic sample D2 (B.), whereby the isolines represent different values of the dimensionless parameters $\zeta$ and $\eta$ determined from Equations in (3.35). In the the vicinity of the bounds, the dimensionless storage capacity $\zeta$ are close to each other for decreasing phase shift [169]. Furthermore, it is obvious that the bounds approach each other for decreasing phase shift and form a tail of the solution space.

The data points in Figure 3.16 (A.-B.) refer to the measured attenuation and phase shift of the pressure signals. It can be seen that the data points are often 


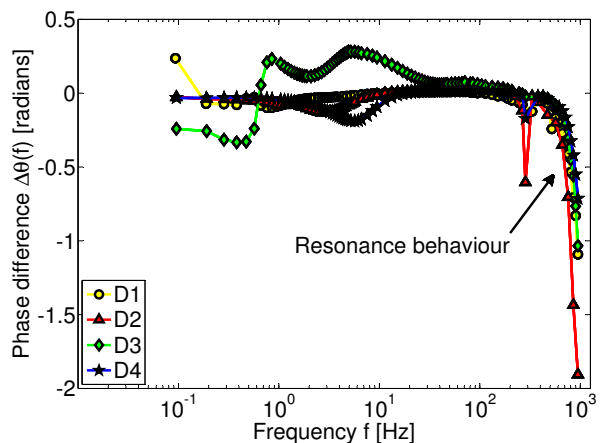

A.)

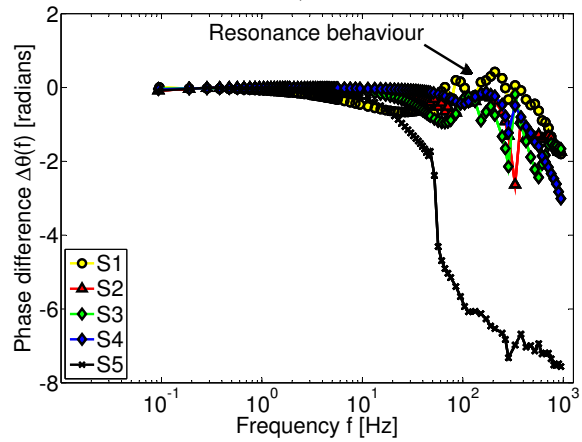

C.)

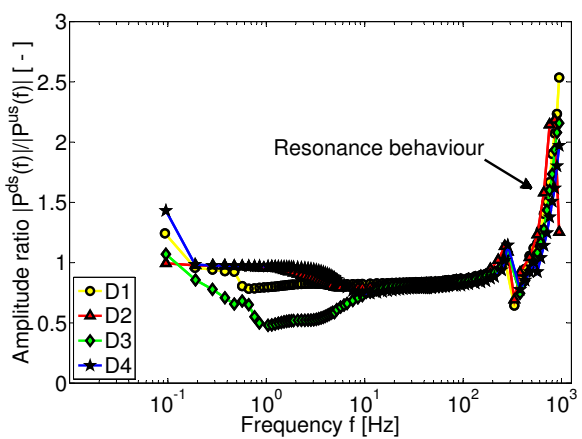

B.)

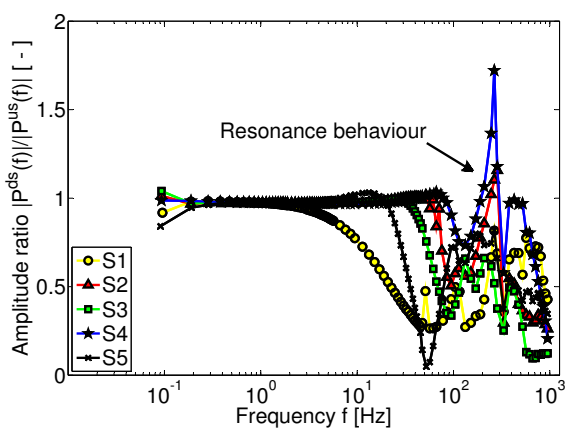

D.)

Figure 3.14: Development of frequency-dependent phase difference and amplitude ration of the synthetic (A. - B.) and sintered sample (C. - D.) within the investigated frequency range of 0.1 till $1000 \mathrm{~Hz}$. 


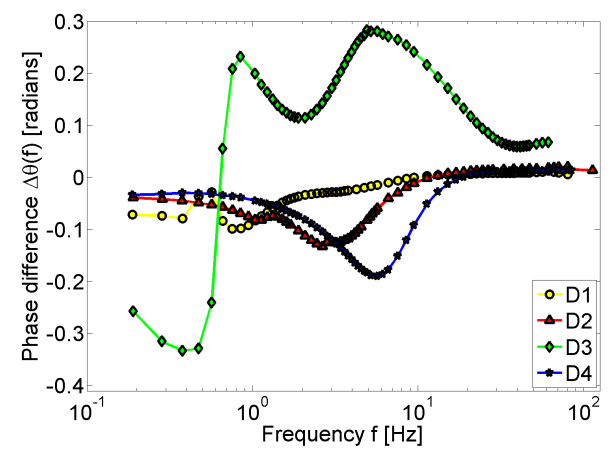

A.)

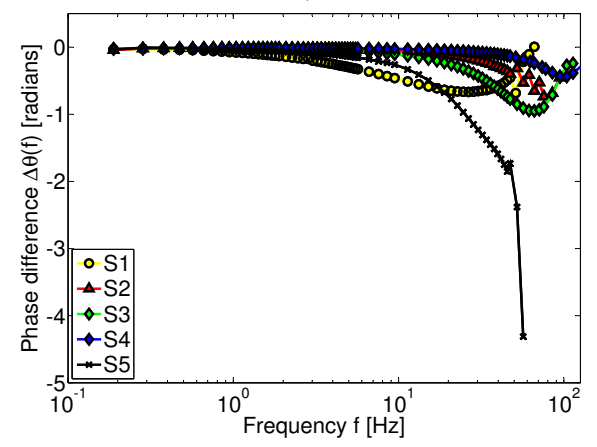

C.)

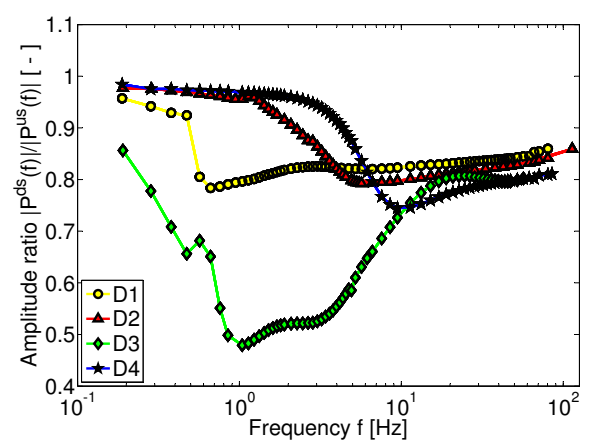

B.)

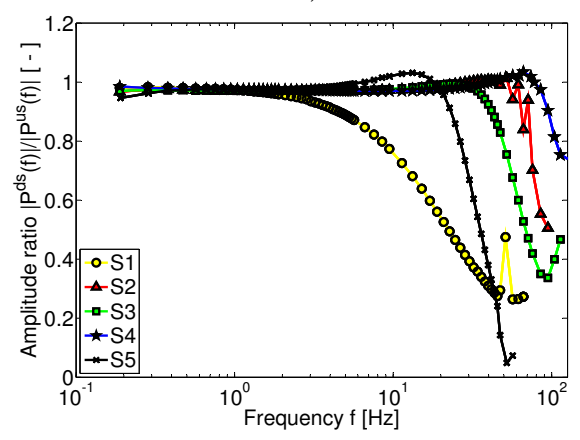

D.)

Figure 3.15: Development of frequency-dependent phase difference and amplitude ration of the synthetic (A. - B.) and sintered sample (C. - D.) within the frequency range of 0.2 till $80 \mathrm{~Hz}$, where resonance effects are negligible small. 


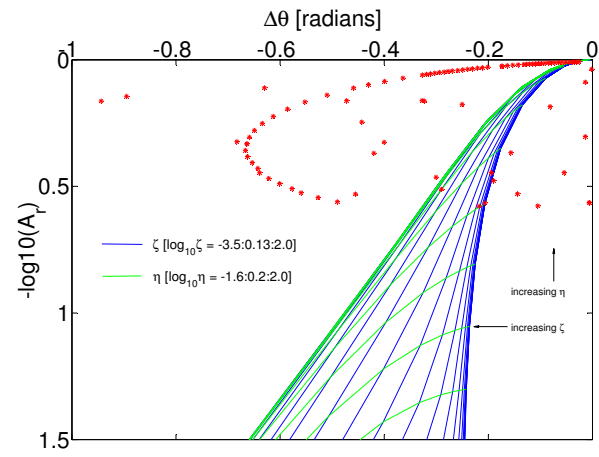

A.)

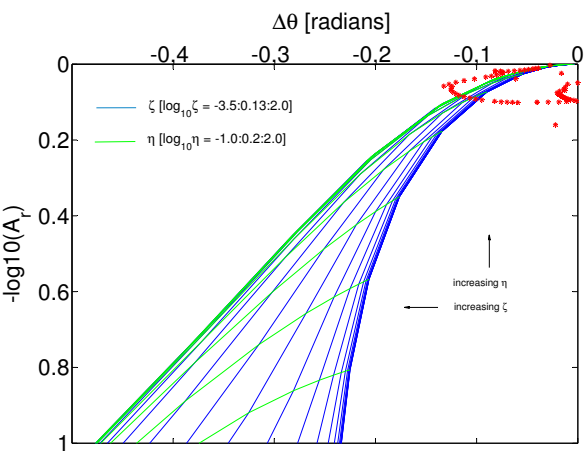

B.)

Figure 3.16: Graphical representation of the analytical solution of the 1-dim diffusion equation for oscillatory flow through an isotropic porous sample in the dimensionless domain of amplitude ratio $A_{r}$ versus phase shift $\Delta \phi$ for: A.) the sintered sample S1 and B.) the synthetic sample D2 consisting of one capillary tube with a diameter $0.75 \mathrm{~mm}$.

not in the solution domain, which can cause deviations in the determination of of the hydraulical parameters.

Figure 3.17 shows the determined hydraulical parameters (for instance the permeability, the storage capacity and the diffusion ceofficient) for a wide frequency range from $0.1 \mathrm{~Hz}$ to $1000 \mathrm{~Hz}$ of the investigated samples. It can be seen the hydraulical parameters show jumps at certain frequencies due to numerical instabilities. Note, that the frequency-dependent inertial effects, which dominantly occur at high frequencies, are not considered in the solution of the diffusion equation, shown in 3.2.3. For a proper determination of the frequency-dependent hydraulical parameters, the coupled set of partial differential equations, presented in subsubsection 3.2.3 1-dim quasi-static case - frequency domain - subsubsection 3.2.3 1-dim dynamic case - frequency domain have to be solved.

Table 3.3 and 3.4 summarize the results for selective frequencies, where the inertial effects are assessed to be small and a good agreement between low-frequency permeabilities obtained from pore oscillation tests and intrinsic permeabilities gained from classical Darcy experiments or analytically derivated permeabilities 


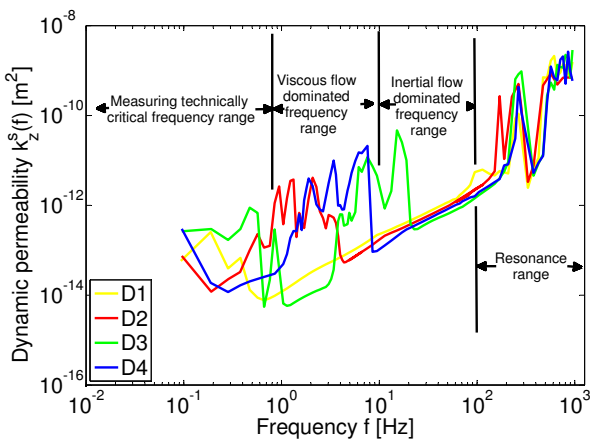

A.)

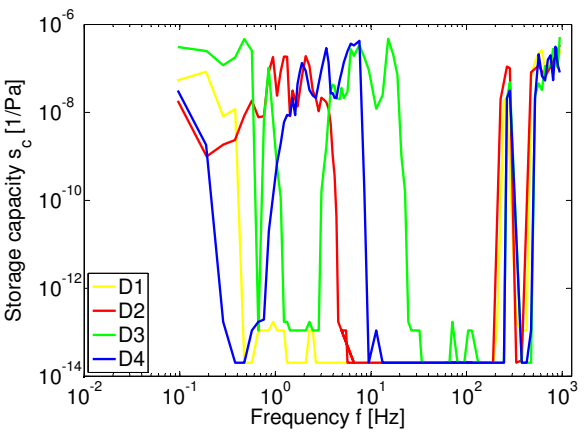

C.)

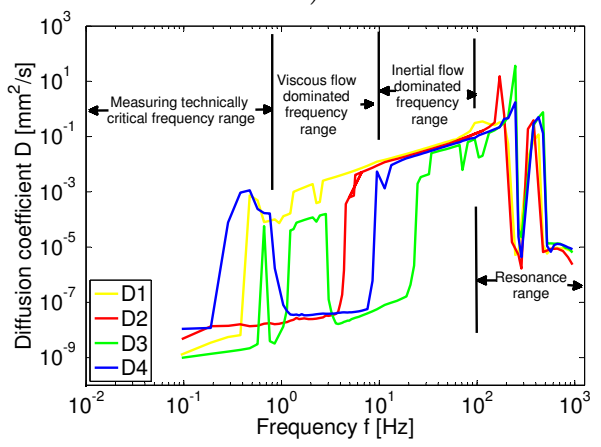

E.)

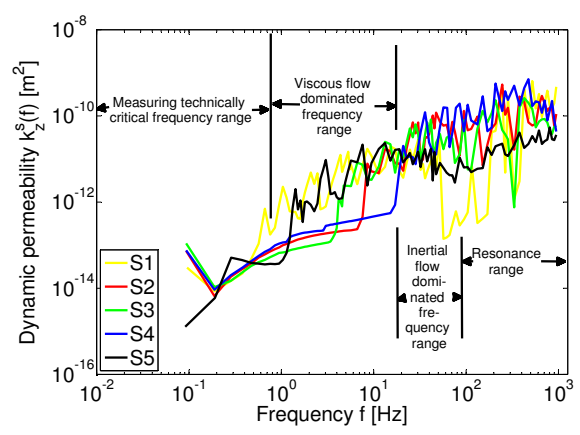

B.)

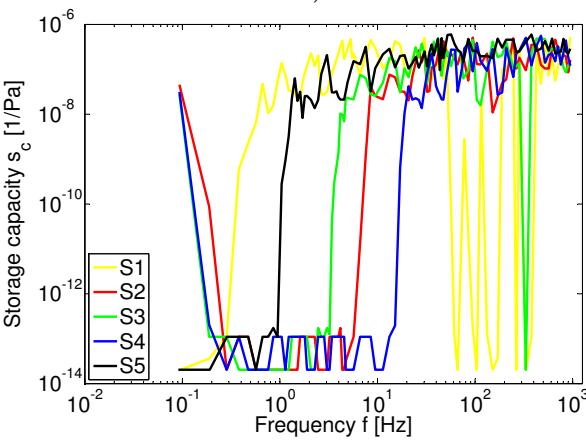

D.)

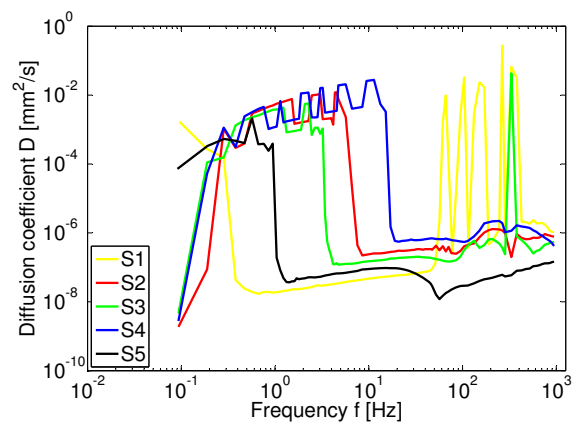

F.)

Figure 3.17: Intrinsic permeabilities (A.-B.), storage capacity (C.-D.) and diffusion coefficient (E.-F.) of the investigated synthetic samples and sintered samples as function of the oscillation frequency. The left panel illustrates the results for the synthetic samples and the right panel for the sintered samples. 
Table 3.3: Comparison of results obtained from analytical solutions and pore pressure oscillation experiments at a specific frequencies.

\begin{tabular}{|c|c|c|c|c|c|}
\hline Parameter & Unit & D1 & D2 & D3S & $\mathrm{D} 4 \mathrm{P}$ \\
\hline Intr. permeability ${ }^{[A]} k_{z}^{s}$ & {$\left[\mathrm{~m}^{2}\right]$} & $7.81 \mathrm{E}-13$ & $3.9551 \mathrm{E}-12$ & $1.3048 \mathrm{E}-12$ & 4.7363E-12 \\
\hline \multirow[t]{2}{*}{ Effect. porosity ${ }^{[B]} \phi_{0}$} & {$[\%]$} & $1.00 \mathrm{E}-02$ & $2.55 \mathrm{E}-02$ & $1.2625 \mathrm{E}-02$ & $3.25 \mathrm{E}-02$ \\
\hline & & & $3.79 \mathrm{~Hz}$ & & \\
\hline Intr. permeability ${ }^{[C]} k_{z}^{s}$ & {$\left[\mathrm{~m}^{2}\right]$} & $6.58 \mathrm{E}-14$ & $2.08 \mathrm{E}-13$ & $4.49 \mathrm{E}-13$ & $9.84 \mathrm{E}-13$ \\
\hline Storage capacity ${ }^{[C]} s_{c}$ & {$\left[\mathrm{~Pa}^{-1}\right]$} & $2.04 \mathrm{E}-14$ & $5.31 \mathrm{E}-09$ & $2.69 \mathrm{E}-08$ & $3.14 \mathrm{E}-08$ \\
\hline Diffusion coefficient ${ }^{[C]} D$ & {$\left[\mathrm{~mm}^{2}\right]$} & $3.72 \mathrm{E}-03$ & $4.51 \mathrm{E}-08$ & $1.64 \mathrm{E}-08$ & $4.02 \mathrm{E}-08$ \\
\hline Storage parameter ${ }^{[C]} \xi$ & {$[-]$} & $3.20 \mathrm{E}-08$ & 8.34E00 & $49.31 \mathrm{E} 00$ & $42.31 \mathrm{E} 00$ \\
\hline \multirow[t]{2}{*}{ Transport parameter $[C] \eta$} & {$[-]$} & $3.97 \mathrm{E} 00$ & $12.64 \mathrm{E} 00$ & $27.23 \mathrm{E} 00$ & $59.74 \mathrm{E} 00$ \\
\hline & & & $7.57 \mathrm{~Hz}$ & & \\
\hline Intr. permeability ${ }^{[C]} k_{z}^{s}$ & {$\left[\mathrm{~m}^{2}\right]$} & $1.56 \mathrm{E}-13$ & $1.11 \mathrm{E}-13$ & $1.18 \mathrm{E}-11$ & $2.11 \mathrm{E}-11$ \\
\hline Storage capacity ${ }^{[C]} s_{c}$ & {$\left[\mathrm{~Pa}^{-1}\right]$} & $2.04 \mathrm{E}-14$ & $2.04 \mathrm{E}-14$ & $3.40 \mathrm{E}-07$ & $7.57 \mathrm{E}-07$ \\
\hline Diffusion coefficient ${ }^{[C]} D$ & {$\left[\mathrm{~mm}^{2}\right]$} & $8.82 \mathrm{E}-03$ & $6.28 \mathrm{E}-03$ & $3.40 \mathrm{E}-08$ & $5.71 \mathrm{E}-08$ \\
\hline Storage parameter ${ }^{[C]} \xi$ & {$[-]$} & $3.20 \mathrm{E}-05$ & $3.20 \mathrm{E}-05$ & $534.30 \mathrm{E} 00$ & $666.90 \mathrm{E} 00$ \\
\hline Transport parameter $[C] \eta$ & {$[-]$} & $4.75 \mathrm{E} 00$ & $3.38 \mathrm{E} 00$ & 359.10E00 & $604.20 \mathrm{E} 00$ \\
\hline
\end{tabular}

$\overline{[A]}$ : Analytical solution under the assumption of a Poiseuille flow.

$[B]$ : Determined from the geometry of the tube-like pore channel and total volume of the sample.

$[C]$ : Determined from pore pressure oscillation tests.

under the assumption of an Poiseuille flow exist. The results of the pore oscillations test are determined from data points, which are within the solution domain shown in Figure 3.16. 
Table 3.4: Comparison of results obtained from analytical solutions and pore pressure oscillation experiments at a specific frequencies.

\begin{tabular}{|c|c|c|c|c|c|c|}
\hline Parameter & Unit & $\mathrm{S} 1$ & $\mathrm{~S} 2$ & $\mathrm{~S} 3$ & $\mathrm{~S} 4$ & S5 \\
\hline Particle diameter $d_{p}$ & {$[\mathrm{~mm}]$} & $0.4-0.6$ & $0.6-0.8$ & $0.8-1.0$ & $1.0-1.2$ & 3.0 \\
\hline Intr. permeability $[A] k_{z}^{s}$ & {$\left[\mathrm{~m}^{2}\right]$} & $7.72 \mathrm{E}-11$ & $5.38 \mathrm{E}-10$ & $5.99 \mathrm{E}-10$ & $4.58 \mathrm{E}-10$ & $3.95 \mathrm{E}-10$ \\
\hline \multirow[t]{2}{*}{ Effect. porosity $[B] \phi_{0} z$} & {$[\%]$} & 35.11 & 36.79 & 29.48 & 32.32 & 22.31 \\
\hline & & & $30.30 \mathrm{~Hz}$ & & & \\
\hline Intr. permeability ${ }^{[C]} k_{z}^{s}$ & {$\left[\mathrm{~m}^{2}\right]$} & $2.81 \mathrm{E}-11$ & $7.08 \mathrm{E}-11$ & $3.86 \mathrm{E}-11$ & $1.95 \mathrm{E}-11$ & $1.48 \mathrm{E}-11$ \\
\hline Storage capacity ${ }^{[C]} s_{c}$ & {$\left[\mathrm{~Pa}^{-1}\right]$} & 4.99E-07 & $2.55 \mathrm{E}-07$ & $2.23 \mathrm{E}-07$ & $3.65 \mathrm{E}-08$ & $2.47 \mathrm{E}-07$ \\
\hline Diffusion coefficient $[C] D$ & {$\left[\mathrm{~mm}^{2}\right]$} & $6.47 \mathrm{E}-08$ & $3.19 \mathrm{E}-07$ & $1.99 \mathrm{E}-07$ & $6.15 \mathrm{E}-07$ & $6.90 \mathrm{E}-08$ \\
\hline Apparent porosity ${ }^{[C]} \phi_{a}$ & [ ] & $1.90 \mathrm{E}-03$ & $5.57 \mathrm{E}-04$ & $4.88 \mathrm{E}-04$ & $7.98 \mathrm{E}-05$ & $5.40 \mathrm{E}-04$ \\
\hline Storage parameter $[C] \xi$ & {$[-]$} & 784.70 & 400.80 & 351.00 & 57.38 & 338.20 \\
\hline \multirow[t]{2}{*}{ Transport parameter ${ }^{[C]} \eta$} & {$[-]$} & 213.40 & 537.80 & 293.20 & 148.30 & 112.60 \\
\hline & & & $52.07 \mathrm{~Hz}$ & & & \\
\hline Intr. permeability ${ }^{[C]} k_{z}^{s}$ & {$\left[\mathrm{~m}^{2}\right]$} & $3.30 \mathrm{E}-11$ & $7.35 \mathrm{E}-11$ & $3.80 \mathrm{E}-11$ & $4.36 \mathrm{E}-11$ & $8.13 \mathrm{E}-12$ \\
\hline Storage capacity ${ }^{[C]} s_{c}$ & {$\left[\mathrm{~Pa}^{-1}\right]$} & $3.00 \mathrm{E}-07$ & $2.00 \mathrm{E}-07$ & $2.52 \mathrm{E}-07$ & $7.51 \mathrm{E}-08$ & $5.80 \mathrm{E}-07$ \\
\hline Diffusion coefficient ${ }^{[C]} D$ & {$\left[\mathrm{~mm}^{2}\right]$} & $1.27 \mathrm{E}-07$ & $4.24 \mathrm{E}-07$ & $1.74 \mathrm{E}-07$ & $6.67 \mathrm{E}-07$ & $1.61 \mathrm{E}-08$ \\
\hline Apparent porosity ${ }^{[C]} \phi_{a}$ & {$[1]$} & $6.55 \mathrm{E}-04$ & $4.36 \mathrm{E}-04$ & $5.55 \mathrm{E}-04$ & $1.64 \mathrm{E}-07$ & $1.27 \mathrm{E}-03$ \\
\hline Storage parameter $[C] \xi$ & {$[-]$} & 407.80 & 313.80 & 395.30 & 118.10 & 911.80 \\
\hline \multirow[t]{2}{*}{ Transport parameter ${ }^{[C]} \eta$} & {$[-]$} & 148.60 & 324.90 & 167.90 & 196.10 & 35.90 \\
\hline & & & $94.67 \mathrm{~Hz}$ & & & \\
\hline Intr. permeability ${ }^{[C]} k_{z}^{s}$ & {$\left[\mathrm{~m}^{2}\right]$} & $3.92 \mathrm{E}-12$ & $1.31 \mathrm{E}-10$ & $7.97 \mathrm{E}-11$ & $4.43 \mathrm{E}-11$ & $6.36 \mathrm{E}-12$ \\
\hline Storage capacity $[C] s_{c}$ & {$\left[\mathrm{~Pa}^{-1}\right]$} & $1.73 \mathrm{E}-13$ & $5.08 \mathrm{E}-07$ & $4.48 \mathrm{E}-04$ & $9.04 \mathrm{E}-08$ & $2.55 \mathrm{E}-07$ \\
\hline Diffusion coefficient $[C] D$ & {$\left[\mathrm{~mm}^{2}\right]$} & $2.64 \mathrm{E}-04$ & $2.96 \mathrm{E}-07$ & $2.04 \mathrm{E}-07$ & $5.64 \mathrm{E}-07$ & $2.86 \mathrm{E}-08$ \\
\hline Apparent porosity ${ }^{[C]} \phi_{a}$ & {$[-]$} & $3.78 \mathrm{E}-10$ & $1.11 \mathrm{E}-03$ & $9.81 \mathrm{E}-04$ & $1.98 \mathrm{E}-04$ & $5.58 \mathrm{E}-04$ \\
\hline Storage parameter $[C] \xi$ & {$[-1$} & $2.72 \mathrm{E}-04$ & 799.20 & 705.30 & 142.10 & 401.20 \\
\hline Transport parameter $[C] \eta$ & {$[-1$} & $9.63 \mathrm{E}-01$ & 318.50 & 193.70 & 107.70 & 15.45 \\
\hline
\end{tabular}

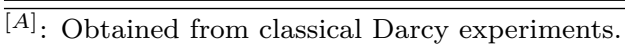

$[B]$ : Determined from bulk density of the produced samples.

$[C]$ : Obtained from pore pressure oscillation tests.

Figure 3.18 shows a direct comparison of the permeabilities determined from pore pressure oscillation tests and classical Darcy experiments by means of bar diagrams. The corresponding oscillation frequency is given by the first numbers in the brackets on the top of the bars. The second number refers to the different glass bead parameter or to the tube diameter of the pore channels. Note, that the determined permeabilities result from different measuring and analysis methods, but nevertheless the permeabilities obtained from pore pressure oscillation tests at specific frequencies are in the same order of magnitude compared to the permeabilities from classical Darcy experiments. On the one hand, it must be ensured, that the data points for the measured attenuation and phase shift of the 


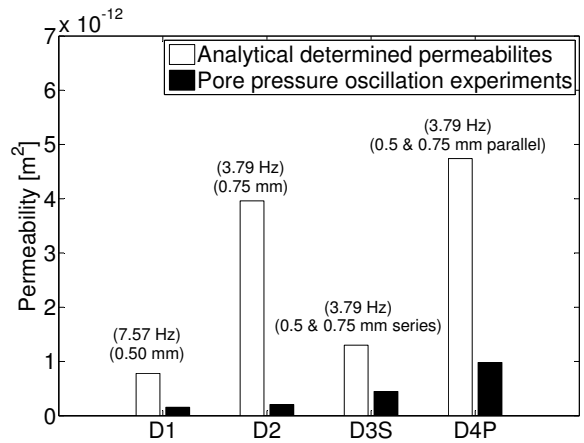

A.)

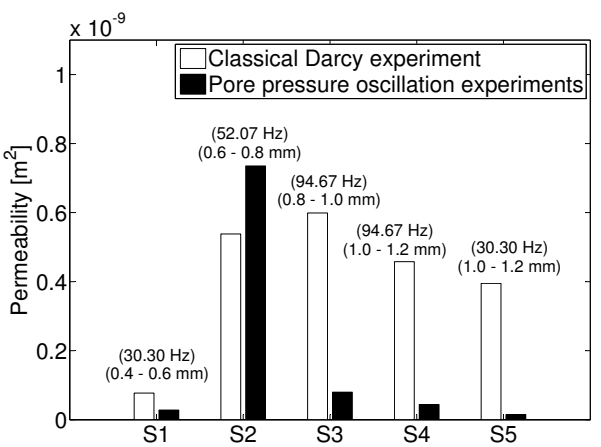

B.)

Figure 3.18: Comparison of permeabilities determined from the pore pressure oscillation test and classical Darcy experiments (or analaytical determined permeability values) for: A.) synthetic samples consisting of capillary and B.) sintered glass bead samples.

pressure signals are within the solution domain of the 1-dim. diffusion equation, on the other hand a proper frequency have to be chosen to avoid inertial effects, which highly affect the intrinsic permeability.

\subsubsection{Amplitude sweep tests}

In addition to the frequency sweeps, amplitude sweep tests have been performed formed to assess the effect of excitation amplitude on the pressure development in the up- and downstream reservoir. The excitation signal is used for the oscillation of the latex membrane or piston plate, respectively. Figure 3.19 shows the amplitude ratio (A.,C.) and phase difference (C.,D) as a function of the excitation signal at specific frequencies. Figure 3.19 (A.,B.) demonstrate the results obtained from measurements, when the measuring cell is completely filled with water, whereas Figure 3.19 (C.,D.) presents the results, when the measuring cell is filled with distilled water up to certain water level. In this specific case, the distance between the water surface and upper pressure transducer in the downstream reservoir has been chosen at $37.5 \mathrm{~mm}$. In both investigated cases, it can be seen that the amplitude ratio and phase difference remain almost constant in dependence on the excitation amplitude, which indicates that non linearity effects in the pressure field within the measuring cell are negligible small, and only occur in the latex membrane in the form of higher mode oscillations. The 
amplitude ratios in Figure 3.19 (C.) are smaller compared to the results shown in Figure 3.19 (C.) due to signal damping in the downstream reservoir. It should be noticed, that the signal-to-noise ratio decreases strongly, especially of the pressure transducer in the downstream reservoir, when the measuring cell is only filled up to certain height. At very low and high frequencies, the useful signal go under into noise, which considerably makes the signal analysis difficult. To overcome these problems, excitation signals with higher amplitudes has to be used. In this case the system becomes unstable due to changes in water level, and the pressure values do not fluctuate around a constant value.

\subsection{Conclusion}

In summary, we have demonstrated that the pore pressure oscillation method can be applied to high-permeable artificially produced samples with permeabilities in the order $10^{-13}-10^{-9} \mathrm{~m}^{2}$ consisting of sintered glass beads or capillary tubes. A good agreement between permeabilities determined from the pore pressure oscillation tests and permeabilities obtained from classical Darcy experiments or analytical determined permeabilities could be achieved. It should be taken into account, that the compared permeabilities are determined from different measuring and analysis methods. Nevertheless, the compared permeabilities are in same order of magnitude, although the permeability change due to inertial losses in the solution 1-dim diffusion Equation (3.27) are not taken into account. Future work should therefore include the inertial effects in the calculation of the permeability from the pore pressure oscillation experiments in both time and frequency domain. The future challenge will be to propose a proper solution for the coupled hydro-mechanical problems formulated in subsubsection 3.2.3 1-dim quasi-static case - frequency domain, subsubsection 3.2.3 1-dim dynamic case time domain and subsubsection 3.2.3 1-dim dynamic case - frequency domain, which takes besides the inertial effects, also the measured amplitude ratios and phase differences of the pressure signals in the up- and downstream reservoir into account. However, the selection of an appropriate frequency for the determination of the intrinsic permeability is still a challenge, and can differ from sample to sample. In many cases, the measured amplitude ratios and phase differences are beyond the solution domain of the 1-dim diffusion equation, which can lead to considerable deviations in the results. In this respect, the use of different pore fluids with higher viscosities, such as silicone oil, can help not only to change the amplitude ratios or phase differences but also to produce a frequency shift of the inertial dominated flow regime towards higher frequencies. Opposed these advantages, the high compressibility of the silicone oil can lead to further measuring uncertainties. Moreover, from a technical point of view the reduction of 


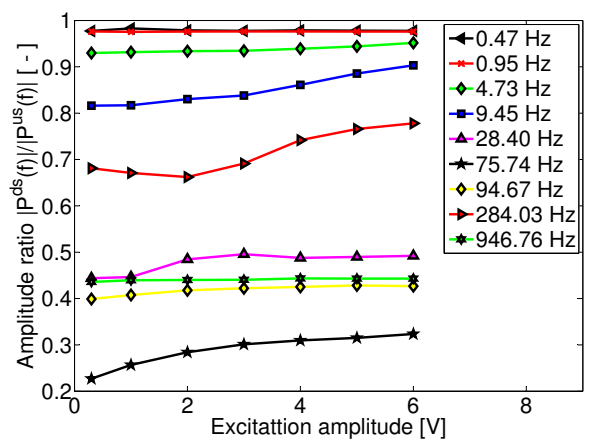

A.)

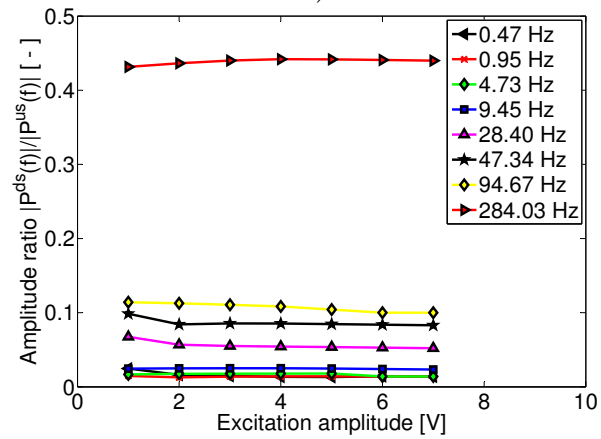

C.)

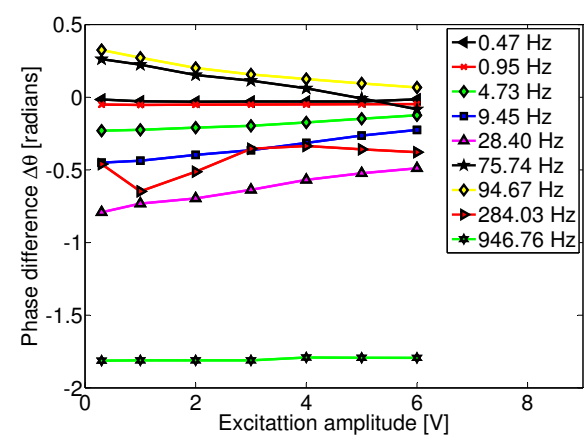

B.)

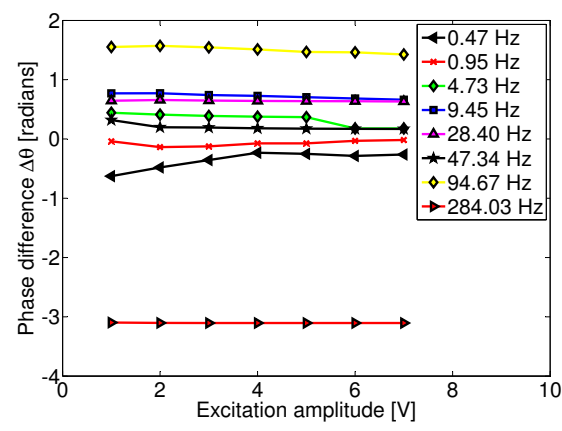

D.)

Figure 3.19: Amplitude sweep tests of sintered sample S1 at different set up configurations. The measuring cell has been either filled completely filled with distilled water (A.-B.) or up to a certain water level (C.-D.). Amplitude ratio (A.,C.) and phase differences (C.,D.) as a function of the amplitude of the excitation signal which is used for oscillation of the latex membrane. 
the downstream reservoir can improve the measuring accuracy of pore pressure oscillation method as indicated in Equation (3.32). In amplitude sweep tests, we have demonstrated that the measured amplitude ratio and phase differences are independent from the excitation amplitudes used to oscillate the latex membrane and non-linearity effects in pressure field are negligible.

\subsection{Acknowledgement}

We acknowledge support from the research program of the Stichting voor Fundamenteel Onderzoek der Materie (FOM), which is financially supported by the "Nederlandse Organisatie voor Wetenschappelijk Onderzoek" (NWO), (project number: 09iPOG14-2).

\subsection{Appendix}




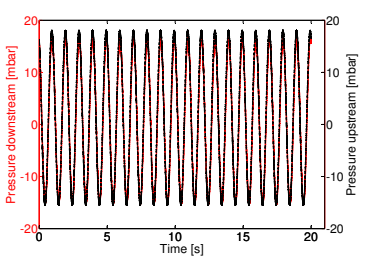

A.)

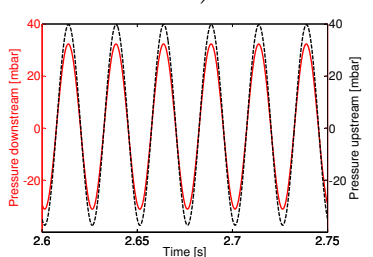

C.)

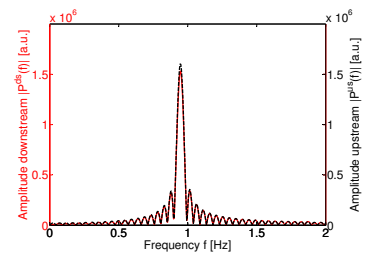

B.)

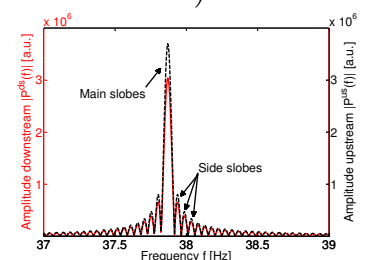

D.)

Figure 3.21: Pressure-time signals of the sintered sample D2 at 0.95 (A.) and $37.87 \mathrm{~Hz}$ (B.) with corresponding amplitude spectra (B. and D.).

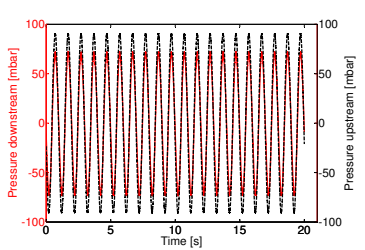

A.)

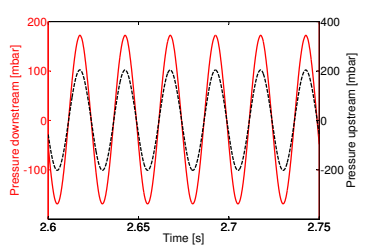

C.)

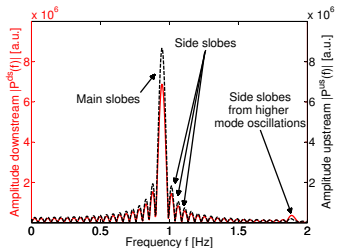

B.)

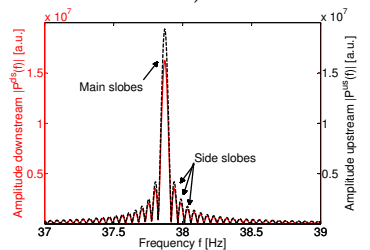

D.)

Figure 3.20: Pressure-time signals of the sintered sample D1 at 0.95 (A.) and $37.87 \mathrm{~Hz}$ (B.) with corresponding amplitude spectra (B. and D.). 


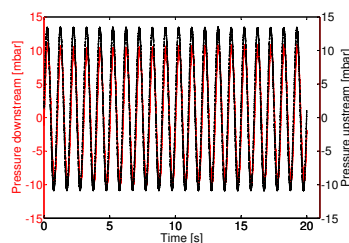

A.)

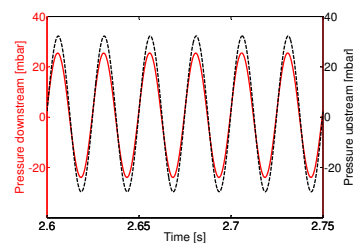

C.)

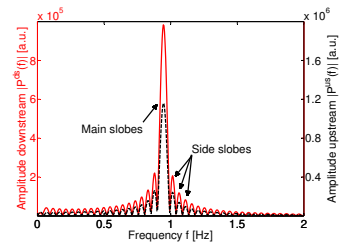

B.)

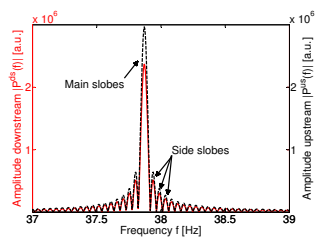

D.)

Figure 3.22: Pressure-time signals of the synthetic sample D3 (in series connected tubes) at 0.95 (A.) and $37.87 \mathrm{~Hz}$ (B.) with corresponding amplitude spectra (B. and D.).

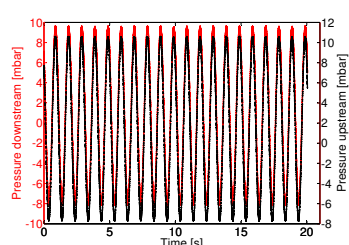

A.)

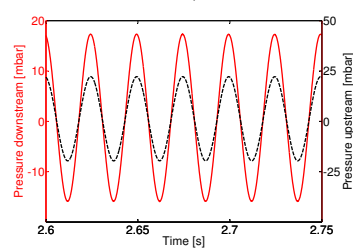

C.)

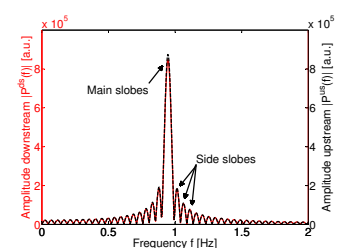

B.)

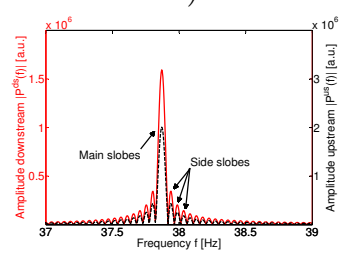

D.)

Figure 3.23: Pressure-time signals of the synthetic sample D4 (in parallel connected tubes) at 0.95 (A.) and $37.87 \mathrm{~Hz}$ (B.) with corresponding amplitude spectra (B. and D.). 


\section{CHAPTER 4}

\section{Wave Propagation in Sintered Porous Glass Bead Systems: Low-Frequency range}

In this paper, we present an experimental and theoretical study of ultrasonic wave propagation in water-saturated artificial porous samples composed of sintered glass beads. In ultrasound experiments, the transmission method is used to determine frequency-dependent properties of the samples. Here, we focus mainly on the coherent deterministic pulse, which describes the macroscopic properties and arrives at the leading edge of the received signal. Different excitation signals, such as a Ricker wavelet, square wave impulse or sinus-burst signal at different frequencies are tested. The dispersion properties, like phase velocity $c_{P h}$, specific attenuation $1 / Q$, normalized attenuation $\hat{\alpha}$, and power spectra or spectrograms of the investigated samples are analyzed for the different excitation signals. Essential structural parameters, such as porosity and tortuosity, which highly influence the acoustical wave propagation in sintered glass bead samples are determined from $\mu X R C T$ analysis. Depending on the sintering treatment, the investigated sintered samples showed different tortuosity and porosity values. Besides the determination of macroscopic properties, the different microstructures at the contact-level of the beads, such as the neck formation of the particles as a result of the sintering procedure are analyzed qualitatively and quantitatively in detail. The neck formation highly influences the macroscopic stiffness of the solid skeleton, and 
thus the acoustical characteristics of the samples.

Furthermore, dry ultrasound and permeability experiments are performed to determine the elastic and hydraulic properties of the sintered glass beads. The measured intrinsic permeability and determined elastic modulus as well as the voxel-based tortuosity and porosity values are used as input parameters in the approved Biottheory to predict the frequency-dependent phase velocity, (specific) attenuation, and Time Of Flight (TOF) of the different wave types. The analytical predictions are compared with ultrasonic experiments and a good agreement between the predicted and measured wave velocities of the fast P1-wave is found for the different samples, when the velocities are determined in the time domain. In contrast to this, the predicted and measured specific and normalized attenuations of the coherent pulses differ qualitatively and quantitatively considerably from each other.

\subsection{Introduction}

The investigation of acoustic wave propagation in fluid-filled porous media is of great interest in many research fields, such as gas or oil explorations in porous rock formations or it can be used in medical applications, for the early diagnosis of osteoporosis. It is a skeletal disease, and connected to loss of bone density as well as changes of the bone structure, whereby the bone matrix is filled with bone mark. The propagating acoustic wave is very sensitive to the micro- and macroscopic features of the fluid-filled porous bulk medium, and thus for instance, can be used to monitor the bone status $[84,132,172,185]$ or to explore gas or oil deposits in deep rock layers [149, 153, 176].

The acoustic wave properties are further strongly affected by internal physical interactions between the solid skeleton and the pore fluid $[153,173]$. The wellknown Biot $[24,25]$ theory has become to be the most common model to describe the acoustical wave propagation in fluid-saturated porous media. Biot $[24,25]$ predicts two compressional waves as a result of coupling and decoupling processes between the solid frame and the pore fluid, in addition to the propagation of a shear wave. Depending on the frequency of the propagating wave, the coupling between the fluid and solid, which is responsible for the propagating of the fast P1wave, is either mainly caused by viscous shear forces of the pore fluid or by inertial effects, often referred as added mass effects, which result from the tortuous pore channels $[87,88]$. The relative motion between both constituents required for the propagation of the slow Biot-wave is ensured in the high-frequency regime, when the frequency-dependent viscous skin depth $\delta$, formulated in Equation (3.16) is smaller than characteristic pore radius $r_{\text {pore }}$. Figure 4.1 (A.) illustrates the valid 


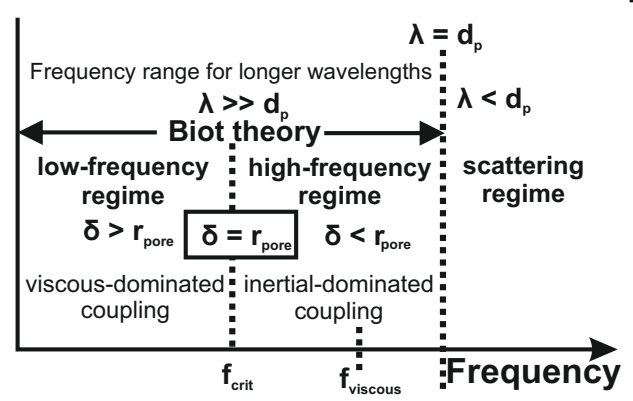

A.)

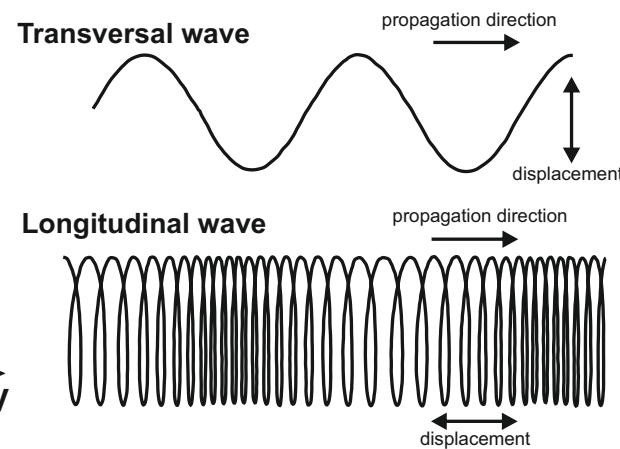

B.)

Figure 4.1: Valid frequency range (A.) of Biot's theory according to Hughes et al. [87] and simplified illustration of wave modes, which are predicted in the classical bi-phase Biot theory (B.) [140].

frequency range for the Biot theory. However, the Biot theory, which is based on a macroscopic approach, is limited to wavelengths, which are much greater than characteristic pore or particle diameter $\left(\lambda \gg d_{p}\right)$, respectively [87]. For $\lambda$ comparable to several $d_{p}$, where spatial inhomogeneities on a microscopic-level within a propagating porous medium introduce scattering effects, the Biot-theory fails. The propagating (multiple-) scattered waves have a diffusive character, and are therefore often described by diffusion models [92, 135-137, 139, 152].

Another major disadvantage of the Biot theory is that the model requires various input parameters for the prediction of the phase velocities and specific attenuations of the different wave types [167]. In this case, $\mu \mathrm{XRCT}$ analysis is an efficient tool, which provides the determination of further essential macro- and microscopic influence parameters and thus can make an important contribution to understand the wave propagation in fluid-saturated media.

Successful application of Biot's theory to cancellous [84, 88, 172, 192] or trabecular [184] bone saturated with water or bone marrow or to water-saturated polyurethane [28] and aluminium foams [70, 91, 154], sedimentary rocks [176] or sands [30] is reported elsewhere. However, in most cases only the fast P1-wave could be detected from broadband ultrasound experiments. The slow P2-wave is highly damped or superimposed by (multiple-) scattered waves.

In this paper, we present experimental ultrasound results on water-saturated sintered glass bead samples, which show high permeabilities (in the order of $10^{-11}$ $10^{-9} \mathrm{~m}^{2}$ ) and open pore structure, and their successful comparison to theoretical predictions according to Biot's theory. Compared with natural rock samples, 
the artificially produced sintered glass beads samples are characterized by their simple and repeatable pore geometry. Moreover, the acoustical properties, like intrinsic permeability and tortuosity, which determine the low- and high-frequency coupling between pore fluid and skeleton frame, is influenced by the choice of glass bead sizes and sintering treatments. In this way, different sintered glass bead samples were produced and the Biot theory can be checked for it's range of validity for different structures. An additional advantage of sintered glass bead samples is the high gray-scale contrast to the pore space, which makes the use of contrast media superfluous and well simplifies the segmentation procedure during $\mu \mathrm{XRCT}$ analysis, cf. [69]. Moreover, the relatively simple structure of the skeleton frame helps to extract and determine the microscopic inter-particle contact areas, which significantly influences the propagation of high-frequency incoherent part consisting of (multiple-)scattered waves.

In this work, we focus on the coherent part of the received ultrasound signals, which corresponds to the fast P1-wave, arising from the mechanical coupling between the fluid and solid phase due to inertial effects. The slow Biot-wave caused by the relative motion is highly damped and/or can be superimposed by (multiple-) scattered ultrasound waves, and thus will be studied in detail elsewhere.

In section 4.2 the Biot theory is introduced and section 4.3 deals with the application of the Biot theory on different sintered samples. For the application of Biot's theory the input parameters for the sintered samples are determined from $\mathrm{XRCT}$ analysis, dry ultrasound and stationary permeability experiments. In section 7.3 the immersion ultrasound broadband experiments are presented. Here, the experimental procedure and is described and the Spectral Ratio Technique (SRT) is introduced. The theoretical and experimental results are presented and discussed in section 4.5. The study is concluded in the final section 4.6.

\subsection{Biot theory}

In this section essential features of Biot's model [24, 25] are briefly introduced. Furthermore, the field equations, which describe the acoustical wave propagation in fully-saturated porous media, are set up, and the influencing parameters are discussed. Based on the field equations, the dispersion relations for the propagation of longitudinal and transversal waves are derived using an harmonic approach for waves, and finally discussed in terms of their low- and high-frequency limits.

\subsubsection{Field equations}

The Biot theory is a macroscopic approach [101] to describe wave propagation in fluid-saturated porous media. Macroscopic parameters, like the bulk and shear 
moduli of the skeletal frame ( $K$ and $G$ ), are expressed in terms of the properties of the constituents, of which the skeleton is made of $\left(K^{s}, E^{s}, G^{s}\right)$ and microstructure of the bulk fabric, for example tortuosity $(\alpha)$ of the pore channels.

An essential factor of Biot's theory is the prediction of two types of compressional waves, which are known as fast (P1) and slow (P2) wave. The P2-wave is also referred as slow Biot-wave [172].

The fast P1-wave arises from the coupling between the solid and fluid phase, when both constituents move in phase. In contrast to this, the slow P2-wave results from the out-of-phase movement of both constituents. Depending on the frequency of the fast P1-wave, the coupling between the fluid and solid phase arises from different physical mechanisms, cf. Figure 4.1 (A.).

The low-frequency regime is defined as the case, when the frequency of the fast P1-wave is lower than the critical frequency $\left(\omega<\omega_{\text {crit }}\right)$

$$
\omega_{\mathrm{crit}}=\frac{\eta^{f R} \phi_{0}}{\alpha \rho^{f R} k_{z}^{s}}
$$

Poiseuille flow through micro channel occurs, and the locking between both constituents arises mainly from the fluid viscosity, which leads to the propagation of a fast P1-wave $[24,25,87,104,172]$. In Equation (4.1) $\eta^{f R}$ and $\rho^{f R}$ describe the real effective dynamic viscosity and density of the pore fluid, whereas $\phi_{0}, k_{z}^{s}$ and $\alpha$ represent the initial porosity, intrinsic permeability (in $z$-direction) and tortuosity of the porous material in a certain Representative Volume Element (RVE). The out-of-phase movement, required for the propagation of the slow Biot wave, cannot be realized in the low-frequency regime. Therefore, the slow P2wave cannot propagate in the low-frequency regime and has a diffusive character $\left(\omega<\omega_{\text {crit }}\right)[24,25,87,172]$.

In the high-frequency regime $\left(\omega>\omega_{\text {crit }}\right)$, the viscous coupling mechanism is dominated by inertial effects, which locks the fluid and solid phase together, as necessary for fast P1-wave propagation. Furthermore, the relative motion between the fluid and solid is not more affected by viscous drag forces, so that the slow P2-wave can propagate $[24,25,87,172]$. For the characterization of the transition between the low- and high-frequency regime, Biot [24, 25] has introduced a frequency-dependent complex-valued viscous correction factor $F(\omega)$. According to $[95,104,172]$, the correction factor can be formulated as

$$
F(\omega)=\sqrt{1+\frac{1}{2} i M \omega / \omega_{\text {crit }}}
$$

whereby $M$ describes the shape factor of the grains and is usually taken as $M \approx 1$ [101]. The factor $F(\omega)$ is defined as the ratio between the frequency-dependent 
and stationary damping factor [101]

$$
F(\omega)=\frac{b(\omega)}{b_{0}},
$$

whereby according to Smeulders [2] the stationary damping factor is denoted as

$$
b_{0}=\frac{\eta^{f R} \phi_{0}}{k_{z}^{s}}
$$

According to Steeb [172] the field equations describing the wave propagation in fully-saturated porous media are formulated by

$$
\begin{aligned}
N \operatorname{div} \operatorname{grad} \mathbf{u}_{s}+(A+N) \operatorname{grad} \operatorname{div} \mathbf{u}_{s}+Q \operatorname{grad} \operatorname{div} \mathbf{u}_{f} & =\rho_{11} \ddot{\mathbf{u}}_{s}+\rho_{12} \ddot{\mathbf{u}}_{f} \\
& +b_{0} F\left(\dot{\mathbf{u}}_{s}-\dot{\mathbf{u}}_{f}\right), \\
Q \operatorname{grad} \operatorname{div} \mathbf{u}_{s}+R \operatorname{grad} \operatorname{div} \mathbf{u}_{f} & =\rho_{12} \ddot{\mathbf{u}}_{s}+\rho_{22} \ddot{\mathbf{u}}_{f} \\
& -b_{0} F\left(\dot{\mathbf{u}}_{s}-\dot{\mathbf{u}}_{f}\right)
\end{aligned}
$$

In Equations (4.5) and (4.6) the inertial forces, which are important in the high frequency regime $\left(\omega \gg \omega_{\text {crit }}\right)$, are expressed by $\rho_{12} \ddot{\mathbf{u}}_{\alpha}$ with $\alpha=s, f$ for the solid and fluid phases, respectively. $\rho_{11}, \rho_{12}$ and $\rho_{11}$ are densities, which describe the added mass effects as a result of the tortuous pore structure [172]. The densities, which take the interaction between the fluid and solid phase into account, can be expressed in terms of real densities of constituents $\left(\rho^{f R}, \rho^{s R}\right)$, tortuosity and porosity by

$$
\begin{aligned}
\rho_{11} & =\left(1-\phi_{0}\right) \rho^{s R}-\rho_{12}, \\
\rho_{22} & =\alpha \phi_{0} \rho^{f R} \\
\rho_{12} & =(1-\alpha) \phi_{0} \rho^{f R} .
\end{aligned}
$$

$\rho_{12}$ is the inertial drag that the solid exerts on the fluid and vice versa. $\rho_{11}$ represents the effective density of the solid moving through the fluid and $\rho_{22}$ describes the opposite case [106].

$A, N, Q$ and $R$ are generalized elastic parameters and can be linked to measurable 
quantities via the so-called "Gedankenexperimente" by

$$
\begin{aligned}
P & =\frac{\left(1-\phi_{0}\right)\left(1-\phi_{0}-K / K^{s}\right) K^{s}+\phi K^{s} K / K^{f}}{1-\phi_{0}-K / K^{s}+\phi_{0} K^{s} / K^{f}}+\frac{4}{3} G \\
Q & =\frac{\left(1-\phi_{0}-K / K^{s}\right) \phi_{0} K^{s}}{1-\phi_{0}-K / K^{s}+\phi_{0} K^{s} / K^{f}} \\
R & =\frac{\phi_{0}^{2} K^{s}}{1-\phi_{0}-K / K^{s}+\phi_{0} K^{s} / K^{f}} \\
A & =P-2 N, \\
N & =G,
\end{aligned}
$$

where $K^{s}$ is the intrinsic bulk modulus of the grains, which the solid skeleton is made of and $K^{f}$ is the bulk modulus of the pore fluid, (cf. Biot, Willis and Gassmann [26, 61]). $K$ and $G$ are the bulk and shear modulus of the solid frame. The denominators of the generalized elastic parameters $Q, R$ in the first term of $P$ can be summarized to an effective porosity as follows

$$
\phi^{E}=\phi_{0}+\frac{K^{f}}{K^{s}}\left(1-\phi_{0}-\frac{K}{K^{s}}\right),
$$

where $\phi_{0}$ the initial porosity, cf. $[24,25,101,172]$. Under the assumption that the compressibility of solid skeleton and pore fluid are much higher, compared to the one of the grains $\left(K / K^{s} \ll 1, K^{f} / K^{s} \ll 1\right)$, it follows that $\phi^{E}=\phi_{0}$, and the elastic parameters $A, Q$ and $R$ are simplified to

$$
\begin{aligned}
A & =\frac{1-\phi_{0}}{\phi_{0}} K^{f}+K-\frac{2}{3} G \\
Q & =K^{f}\left(1-\phi_{0}\right) \\
R & =\phi_{0} K^{f} .
\end{aligned}
$$

Assuming a harmonic approach for waves and with the use of the so-called Helmholtz decomposition with potentials, according to Refs. [101, 105, 167, 172], the field Equations (4.5) and (4.6) are separated into a transversal and longitudinal mode and transformed into the frequency domain. In the transversal case the displacement of the medium is perpendicular to the direction of the wave propagation, whereas in the case of the longitudinal mode the displacement of the medium is parallel to the propagation direction, cf. Figure 4.1 (B.). The following sections consider the dispersion relations for both wave types. 


\subsubsection{Transversal mode}

For transversal wave modes the eigenvalue formulation can be written in accordance with [172] in the form

$$
\mathbf{A}_{S} \hat{\boldsymbol{\Psi}}=k_{S}^{2} \mathbf{B}_{S} \hat{\boldsymbol{\Psi}}
$$

with $\hat{\boldsymbol{\Psi}}=\left[\hat{\psi}_{s} \hat{\psi}_{f}\right]^{T}$ and

$$
\mathbf{A}_{S}=\left[\begin{array}{ll}
\hat{\rho}_{11} & \hat{\rho}_{12} \\
\hat{\rho}_{12} & \hat{\rho}_{22}
\end{array}\right] \omega^{2} \quad \text { and } \quad \mathbf{B}_{S}=\left[\begin{array}{cc}
N & 0 \\
0 & 0
\end{array}\right]
$$

$\hat{\mathbf{\Psi}}$ represents the potential vector for transversal waves. $\mathbf{A}_{S}$ and $\mathbf{B}_{S}$ are the inertia and stiffness matrices, whereby frequency-dependent densities

$$
\begin{aligned}
& \hat{\rho}_{12}=\rho_{12}+i b_{0} F / \omega, \\
& \hat{\rho}_{11}=\rho_{11}-i b_{0} F / \omega, \\
& \hat{\rho}_{22}=\rho_{22}-i b_{0} F / \omega,
\end{aligned}
$$

are introduced. The corresponding dispersion relation in the transversal case is a linear equation for $\zeta_{S}=k_{S}^{2}$ and reads

$$
\zeta_{S}(\omega)=\frac{\hat{\rho}_{11} \hat{\rho}_{22}-\hat{\rho}_{12}^{2}}{N \hat{\rho}_{22}} .
$$

In the transversal case the dispersion relation shows one physical solution for the frequency-dependent wave number $k_{S}(\omega)$. The frequency-dependent phase velocity $c_{S}$, attenuation $\alpha_{S}$ and specific attenuation $1 / Q_{S}$ of the shear wave can be computed using the linear dispersion relation (4.24) as follows

$$
c_{S}:=\frac{\omega}{|\operatorname{Re}[\sqrt{\zeta(\omega)}]|}, \quad \alpha_{S}:=|\operatorname{Im}[\sqrt{\zeta(\omega)}]|, \quad \frac{1}{Q_{S}}:=\frac{2|\operatorname{Im}[\sqrt{\zeta(\omega)}]|}{|\operatorname{Re}[\sqrt{\zeta(\omega)}]|} .
$$

\subsubsection{Longitudinal mode}

In the case of compressional wave propagation, the eigenvalue problem can formulated as

$$
\mathbf{A}_{P} \hat{\boldsymbol{\Phi}}=k_{P}^{2} \mathbf{B}_{P} \hat{\boldsymbol{\Phi}}
$$


with $\hat{\boldsymbol{\Phi}}=\left[\hat{\phi}_{s} \hat{\phi}_{f}\right]^{T}$ as the potential vector for longitudinal waves and

$$
\mathbf{A}_{S}=\left[\begin{array}{ll}
\hat{\rho}_{11} & \hat{\rho}_{12} \\
\hat{\rho}_{21} & \hat{\rho}_{22}
\end{array}\right] \omega^{2} \quad \text { and } \quad \mathbf{B}_{P}=\left[\begin{array}{ll}
P & Q \\
Q & R
\end{array}\right],
$$

where $\mathbf{A}_{P}$ and $\mathbf{B}_{P}$ represent the inertia and stiffness matrices in case of longitudinal wave mode. The corresponding dispersion relation for $\zeta_{P}=k_{P}^{2}$ is a quadratic equation and can be written as

$$
\left[P R-Q^{2}\right] \zeta^{2}-\left[P \hat{\rho}_{22}+R \hat{\rho}_{11}-2 Q \hat{\rho}_{12}\right] \zeta+\left[\hat{\rho}_{11} \hat{\rho}_{22}-\hat{\rho}_{12} \hat{\rho}_{12}\right]=0,
$$

where

$$
\Delta=P \hat{\rho}_{22}+R \hat{\rho}_{11}-2 Q \hat{\rho}_{12} .
$$

Equation (4.28) provides two physical solutions for the complex frequency-dependent wave number $k_{P}(\omega)$. Therefore, we obtain two compressional waves, which are known as the fast P1- and the slow P2-wave. The solution for the square of the complex wave number reads

$$
\zeta_{P}(\omega)=\frac{\Delta \pm \sqrt{\Delta^{2}-4\left(P R-Q^{2}\right)\left(\hat{\rho}_{11} \hat{\rho}_{22}-\hat{\rho}_{12} \hat{\rho}_{12}\right)}}{2\left(P R-Q^{2}\right)} .
$$

In analogy to the transversal case the phase velocities, attenuation and the specific attenuation of the fast and slow P-wave can be determined by

$$
\begin{aligned}
c_{P 1, P 2}:=\frac{\omega}{\left|\operatorname{Re}\left[\sqrt{\zeta_{P 1, P 2}(\omega)}\right]\right|}, \quad \alpha_{P 1, P 2} & :=\left|\operatorname{Im}\left[\sqrt{\zeta_{P 1, P 2}(\omega)}\right]\right|, \\
\frac{1}{Q_{P 1, P 2}} & :=\frac{2\left|\operatorname{Im}\left[\sqrt{\zeta_{P 1, P 2}(\omega)}\right]\right|}{\left|\operatorname{Re}\left[\sqrt{\zeta_{P 1, P 2}(\omega)}\right]\right|} .
\end{aligned}
$$

\subsubsection{Low- and high-frequency limits}

For low-frequencies, $\omega \rightarrow 0$, the slow P2-wave vanishes, so that only the fast P1 and shear wave appear. Moreover, the phase velocity becomes independent of 
the frequency and can be written in accordance with [105] as

$$
\begin{aligned}
& \lim _{\omega \rightarrow 0} c_{s}(\omega):=c_{G W, S}=\sqrt{\frac{G}{\left(1-\phi_{0}\right) \rho^{s R}+\phi_{0} \rho^{f R}}}, \\
& \lim _{\omega \rightarrow 0} c_{P 1}(\omega):=c_{G W, P 1}=\sqrt{\frac{K^{s}+\frac{4}{3} \mu^{s}+K^{f} / \phi_{0}}{\rho^{s}+\rho^{f}}} .
\end{aligned}
$$

The low-frequency limit of wave propagation in two-phase systems is described by Gassmann and Wood [61, 105]. Therefore, Equations (4.32) and (4.33) are often named as Gassmann-Wood $(G W)$ limit.

At very high frequencies $(\omega \rightarrow \infty)$, when coupling of fluid and solid is mainly caused by inertial effects due to tortuosity of pore channels, the phase velocity becomes also frequency-independent. For $\alpha \rightarrow 1$ the fluid decouples completely from the solid matrix. According to [121] the high-frequency velocities are given by

$$
\begin{aligned}
c_{P 1, P 2} & =\sqrt{\left(\frac{\Delta_{1} \pm\left[\Delta_{1}^{2}-4\left(\rho_{11} \rho_{22}-\rho_{12}^{2}\right)\left(P R-Q^{2}\right)\right]^{1 / 2}}{2\left(\rho_{11} \rho_{22}-\rho_{12}^{2}\right)}\right)}, \\
c_{S} & =\sqrt{\frac{G}{\rho^{b}-\phi_{0} \rho^{f R} \alpha^{-1}}},
\end{aligned}
$$

where

$$
\Delta_{1}=P \rho_{22}+R \rho_{11}-2 Q \rho_{12}
$$

and

$$
\rho^{b}=\left(1-\phi_{0}\right) \rho^{s R}+\phi_{0} \rho^{f R},
$$

which describes the effective density of the bulk medium.

\subsection{Experimental methods - Application to sin- tered glass beads}

In this section the experimental ultrasound measurements according to the transmission method is presented. Furthermore, the Spectral Ratio Technique (SRT) is introduced, which is used in common ultrasound experiments to characterize the dispersion behavior of the investigated samples. For a better understanding of the frequency-dependent wave propagation the investigated samples are described on different length-scales qualitatively and quantitatively using $\mu \mathrm{XRCT}$ scans. Microscopic and macroscopic influencing parameters are determined, which are 
used as input parameters for the Biot model.

\subsubsection{Specimen}

In this study, three cylindrical sintered glass bead samples with bulk diameters and sample length of $50 \mathrm{~mm}$ are used, see Figure 4.2. The ultrasonic measurements are performed in filtered and de-aired water at room temperature. The water temperature is measured for each experiment and ranged around $22 \pm 0.3^{\circ} \mathrm{C}$.

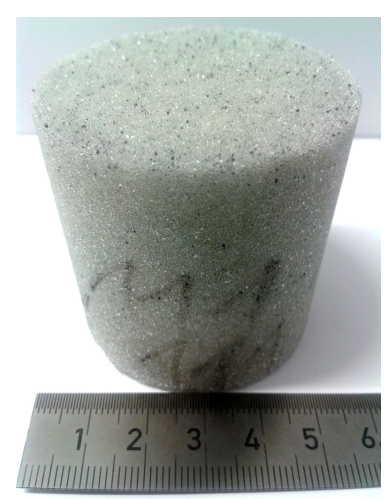

A.)

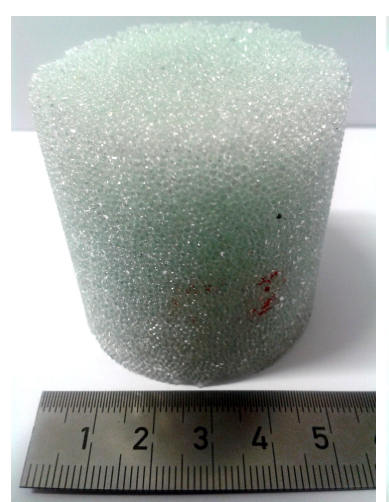

B.)

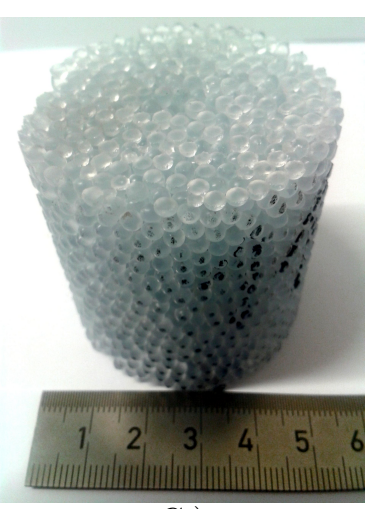

C.)

Figure 4.2: Photographs of investigated sintered glass bead samples with different particle diameters: A.) $\left.d_{p}=0.4-0.6 \mathrm{~mm} \mathrm{B.)} d_{p}=1.0-1.2 \mathrm{~mm} \mathrm{C.}\right) d_{p}=3.0 \mathrm{~mm}$

\section{Porosity, tortuosity and permeability}

Essential material and structural parameters determining the acoustical properties, such as porosity and tortuosity, are examined from $\mu \mathrm{XRCT}$ scans of the sintered glass bead packages, and finally used as input parameters in Biot's theory. For the determination of porosity and tortuosity values, representative volume elements with edge lengths of 1024 voxels in $x$ - and $y$-direction and 2048 voxels in $z$-direction are extracted and analyzed. Figure 4.3 (left) shows exemplary a volume-rendered cuboid after segmentation and labeling procedure for the sintered glass bead sample showing particle diameters between 1.0 and $1.2 \mathrm{~mm}$. The RVEs for the investigated samples are selected such that, on the one hand, they contain as many particles as possible, and on the other hand, they are far away from the outer zones of the entire scanned region. In this way, the impact of the outer zones on the porosity and tortuosity can be minimized. The (geometrical) tortuosity is determined from the centroids of two-dimensional projections 
gained from the $\mu \mathrm{XRCT}$ scans. The permeability of the samples is determined by stationary permeability experiments and verified with Lattice Boltzmann simulations. A detailed description of the morphology, hydraulic and tortuosity determination properties is also described in [54, 69].

The corresponding pore space, depicted in Figure 4.3 (right), exhibits a typical open-pore foam structure indicating that the microstructure is mainly formed in early and intermediate sintering stages where the neck radius between the particles is not too strongly developed, so that the pore spaces remain interconnected.

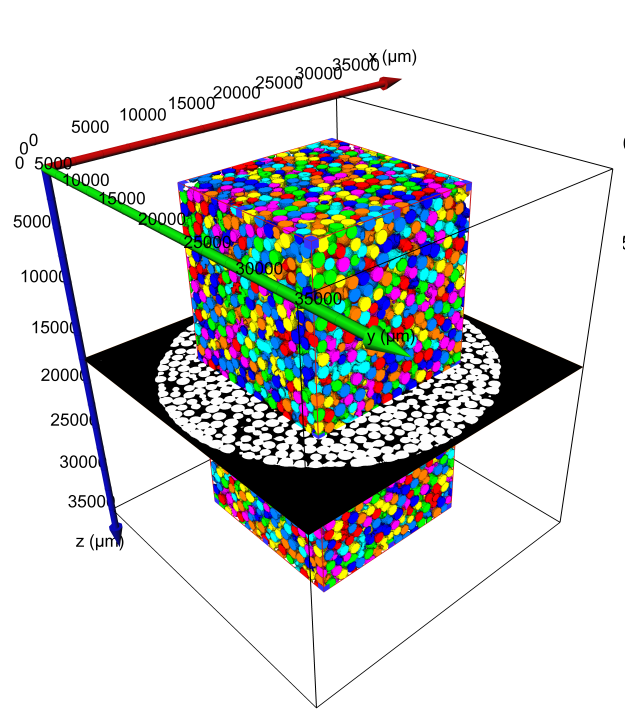

A.)

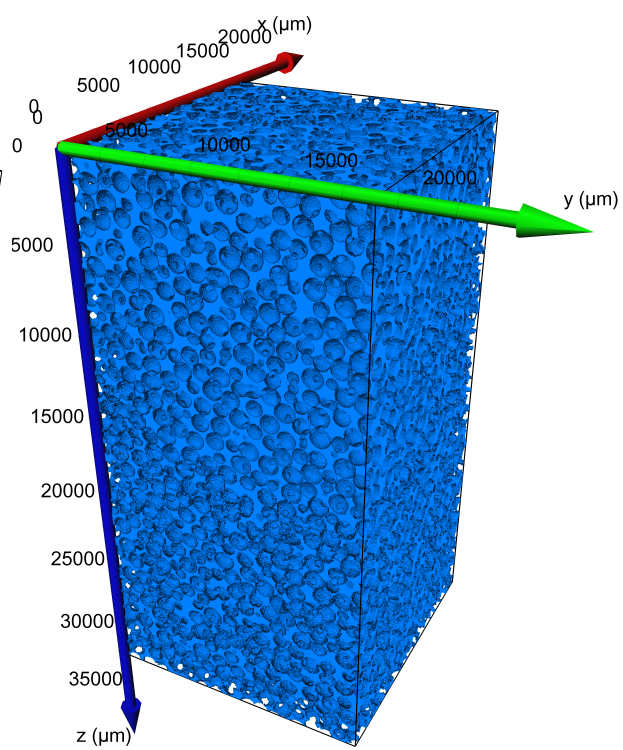

B.)

Figure 4.3: Extracted volume-rendered cuboid with the dimensions 1024 x $1024 \mathrm{x}$ 2048 voxels of sintered porous sample showing particle diameters between 1.0 and $1.2 \mathrm{~mm}$ (A.). The illustration shows the investigated cuboid after segmentation and labeling procedure. The voxel resolution is $16 \mu \mathrm{m}$. The entire scanned region is demonstrated through the $2 \mathrm{D}$ slice. The corresponding pore space shows a typical foam structure (B.).

\section{Sinter neck and elastic parameters of the solid skeleton}

Figure 4.4 shows two binarized glass particles for $d_{p}=1.0-1.2 \mathrm{~mm}$ and $d_{p}=3.0 \mathrm{~mm}$ with the corresponding contact areas resulted from the neck formation (depicted in beige) obtained from $\mu \mathrm{XRCT}$ analysis. This also confirms optically the low deformation of the particles. The particles have not lost their original spherical 
shape after sintering. The optical impressions on the particles of the contact areas are clearly visible.

As it can be seen in Figure 4.4 A.), an equivalent neck diameter $d_{n k}$ is examined, in order to quantify the degree of sintering. The main image processing steps for visualization and quantification of the contact areas between the particles are depicted in Figure 4.4 B.). Note that for the determination of the contact areas the "bin separate" module was used, which is a fast implementation of the socalled watershed separation method. It is not very accurate, but for the sintered glass beads sufficient. Figure 4.5 shows the determined equivalent neck diameter distribution of the investigated samples. In all investigated cases, a monomodal distribution occurs. It can be seen, that the sample with particle diameters of $3.0 \mathrm{~mm}$ exhibits larger values for the neck diameter compared to the samples showing particle diameters of $d_{p}=0.4-0.6 \mathrm{~mm}$ and $d_{p}=1.0-1.2 \mathrm{~mm}$. In the case of the sample with $d_{p}=3.0 \mathrm{~mm}$, the arithmetic mean value of the neck diameter is about $\left\langle d_{n k}\right\rangle=1092 \mu \mathrm{m}$, while the samples with $d_{p}=0.4-0.6 \mathrm{~mm}$ and $d_{p}=1.0$ $1.2 \mathrm{~mm}$ show arithmetic mean values of $\left\langle d_{n k}\right\rangle=196 \mu \mathrm{m}$ and $\left\langle d_{n k}\right\rangle=378 \mu \mathrm{m}$. The reason for this, is that, the $3 \mathrm{~mm}$-sample is in total sintered 1.5 hours longer than the other samples. The longer sintering treatment of the sample with $d_{p}=3.0 \mathrm{~mm}$ is also reflected in the porosity values, cf. Table 4.1 .

The determined inter-particle contact areas on micro-scale influence the macroscopic properties significantly, such as the stiffness of the solid matrix.

The time signals obtained from dry ultrasound measurements confirm this, cf. Figure 4.6. For the determination of elastic properties of skeleton frames composed of sintered glass beads, ultrasound measurements in dry conditions are performed using contact transducers of Panametrics with central frequencies of $100 \mathrm{kHz}$ (type V 1001). For this purpose, the investigated samples are pneumatically fixed ${ }^{2}$ between two transducers, and sounded briefly with square wave impulses showing time durations of $10 \mu$ s. Figure 4.6 A.-C.) shows the time signals of the investigated samples with the corresponding normalized power spectra (D.) obtained from the coherent parts of the received time signals by applying a window in the time domain. One can see from the time signals, that the coherent P1-wave in the case of the sample with $d_{p}=3.0 \mathrm{~mm}$, obviously arrives earlier than the samples with $d_{p}=0.4-0.6 \mathrm{~mm}$ and $d_{p}=1.0-1.2 \mathrm{~mm}$ due to the longer sintering duration, and thus lower porosity values. The $E$-modules of the solid skeleton varies greatly depending on sinter degree, particle size and neck formation. The elastic parameters are listed in Table 4.1. The normalized power spectra of the investigated samples, illustrated in Figure 4.6 D.), show peaks between 80 and

\footnotetext{
${ }^{2}$ with 0.05 bar
} 

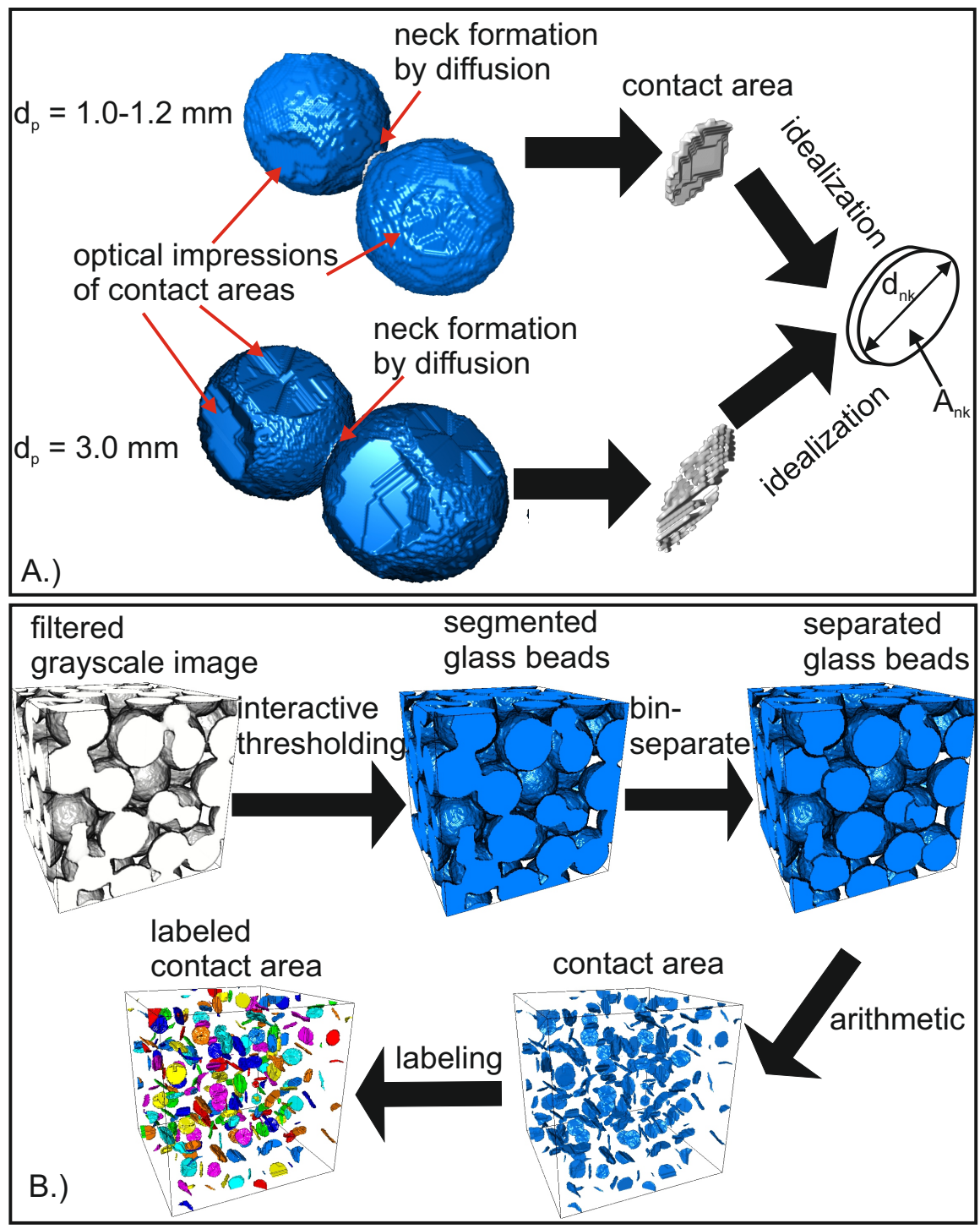

Figure 4.4: A.) Visualization of interparticle contact surfaces and neck formation through the examples of glass beads with particle diameters of $d_{p}=1.0-1.2 \mathrm{~mm}$ and $d_{p}=3.0 \mathrm{~mm}$ after the sintering procedure. After idealization of the interparticle contact areas an equivalent neck diameter $d_{n k}$ is determined. B.) Illustration of the main image processing steps for visualization and quantification of interparticle contact surfaces, which are mainly responsible for the elastic parameters of the solid skeleton composed of sintered glass beads. Note the different scales between Figures (A.) and (B.). 


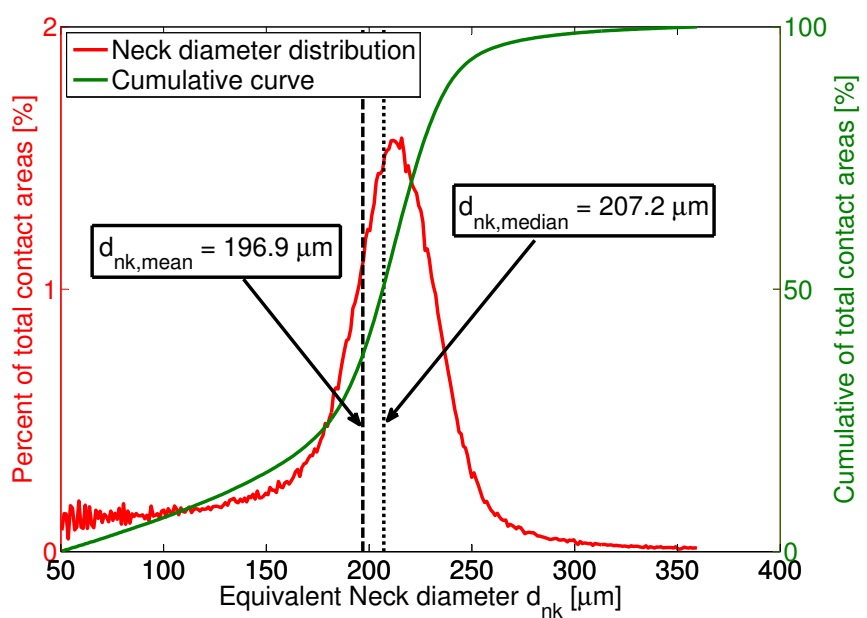

A.)

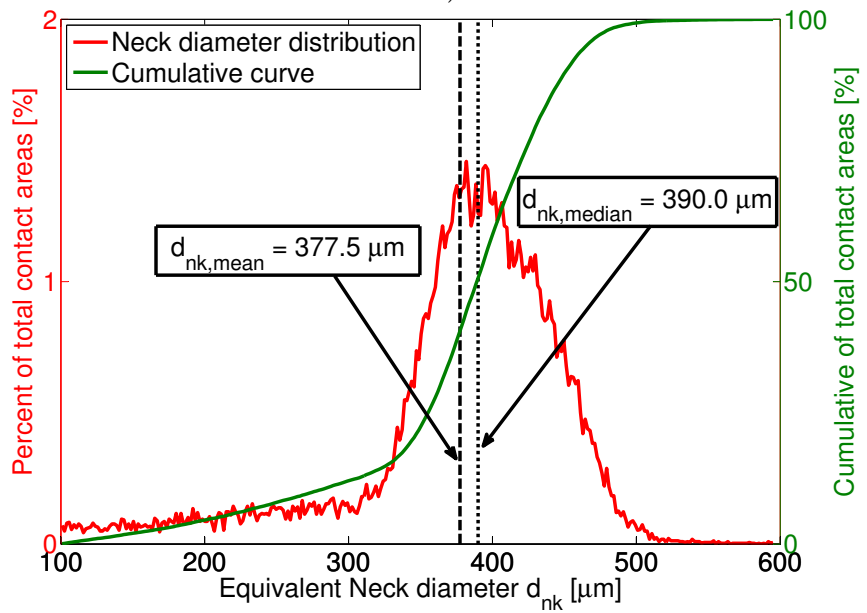

B.)

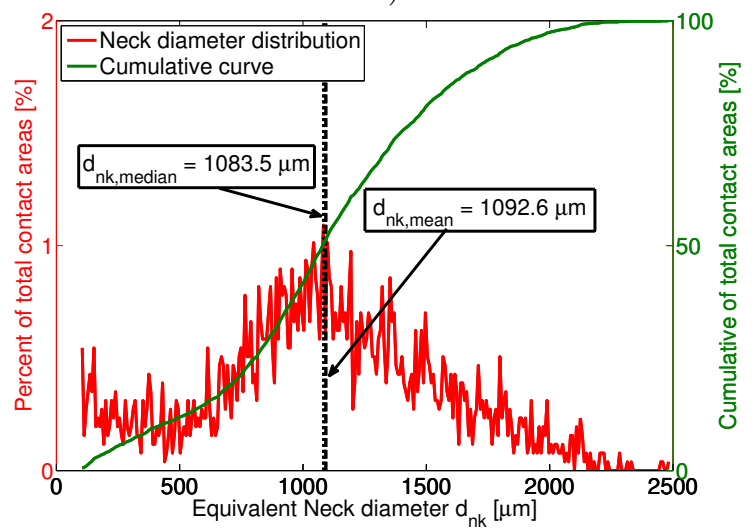

C.)

Figure 4.5: Equivalent neck diameter $d_{n k}$ distribution and corresponding cumulative curves of the investigated samples showing different particle diameter: A.) $\left.\left.d_{p}=0.4-0.6 \mathrm{~mm} \mathrm{B.}\right) d_{p}=1.0-1.2 \mathrm{~mm} \mathrm{C.}\right) d_{p}=3.0 \mathrm{~mm}$. 
$110 \mathrm{kHz}$, which indicates that the square wave impulses of low-frequency content (around $100 \mathrm{kHz}$ ) could easily pass through samples without being distorted much and filtered from the samples.

\section{Pore shape and volume}

The macroscopic acoustical low- and high-frequency coupling parameters, like the intrinsic permeability and tortuosity are highly influenced by the pore shape in micro-scale [71]. Figure 4.7 A.) illustrates the pore spaces between the beads on the basis of the samples with $d_{p}=1.0-1.2 \mathrm{~mm}$ and $d_{p}=3.0 \mathrm{~mm}$ as a result of the sintering procedure obtained from $\mu \mathrm{XRCT}$ analysis. Both samples, depicted in Figure 4.7 A.), show funnel-shaped pore spaces with distinct pore throats, which highly influence the tortuosity and intrinsic permeability in sintered granular systems. The influence of pore throats on the hydraulical properties in sintered porous glass bead systems is reported extensively in [69].

For the quantitative determination of pore sizes of the investigated samples, the pore space is separated and labeled according to Figure 4.7 B.). The resulting pore volume distributions of the investigated samples are shown in Figure 4.8. The pore volume distributions show that the mean pore volume values $\left\langle V_{\text {pore }}\right\rangle$ increase with increasing particle diameter $\left\langle d_{p}\right\rangle$ of the investigated samples despite the fact that the porosity of the $3.0 \mathrm{~mm}$-sample is much lower, cf. Table 4.1. However, the pores are larger and the total number of labeled pore spaces within the investigated cuboid in the case of the $3 \mathrm{~mm}$-sample is much lower compared to the other samples.

The elastic, hydraulical and structural parameters for solid and fluid used as input parameters in Biot's model are summarized in Table 4.1.

\subsection{Experiments}

\subsubsection{Ultrasonic methods}

The experimental setups used for ultrasound measurements in water are illustrated in Figure 4.9. As it can be seen from the illustrations in Figure 4.9, two different configurations are used during ultrasound experiments. 


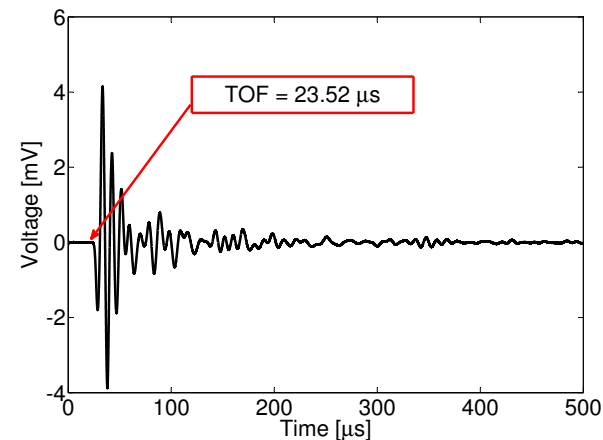

A.)

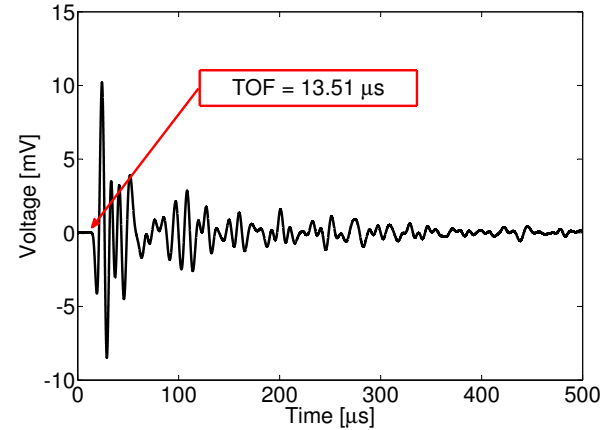

C.)

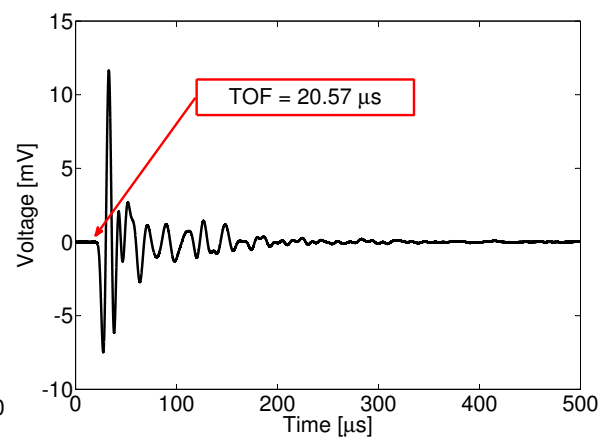

B.)

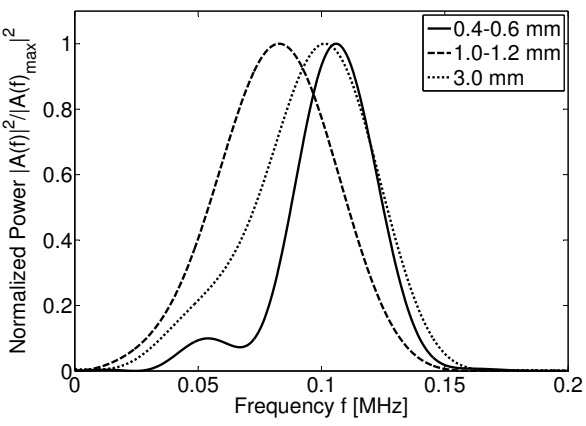

D.)

Figure 4.6: Time signals of different samples obtained from dry measurements (A.) $d_{p}=0.4-0.6 \mathrm{~mm}$, B.) $d_{p}=1.0-1.2 \mathrm{~mm}$, C. $) d_{p}=3.0 \mathrm{~mm}$ ) and corresponding normalized power spectra (D.) obtained from the coherent part of the time signals by FFT. The piezo-electric acoustic transmitter is pulsed at the central frequency of $100 \mathrm{kHz}$ using square wave impulser of Panametrics NDT (model $5077 \mathrm{PR}$ ). For the comutation of the FFT time windows with approxiamtely 10 to $12 \mu \mathrm{s}$ are used. The samples are directly excited using contact broadband transducers of Panametrics NDT (type V1011). 

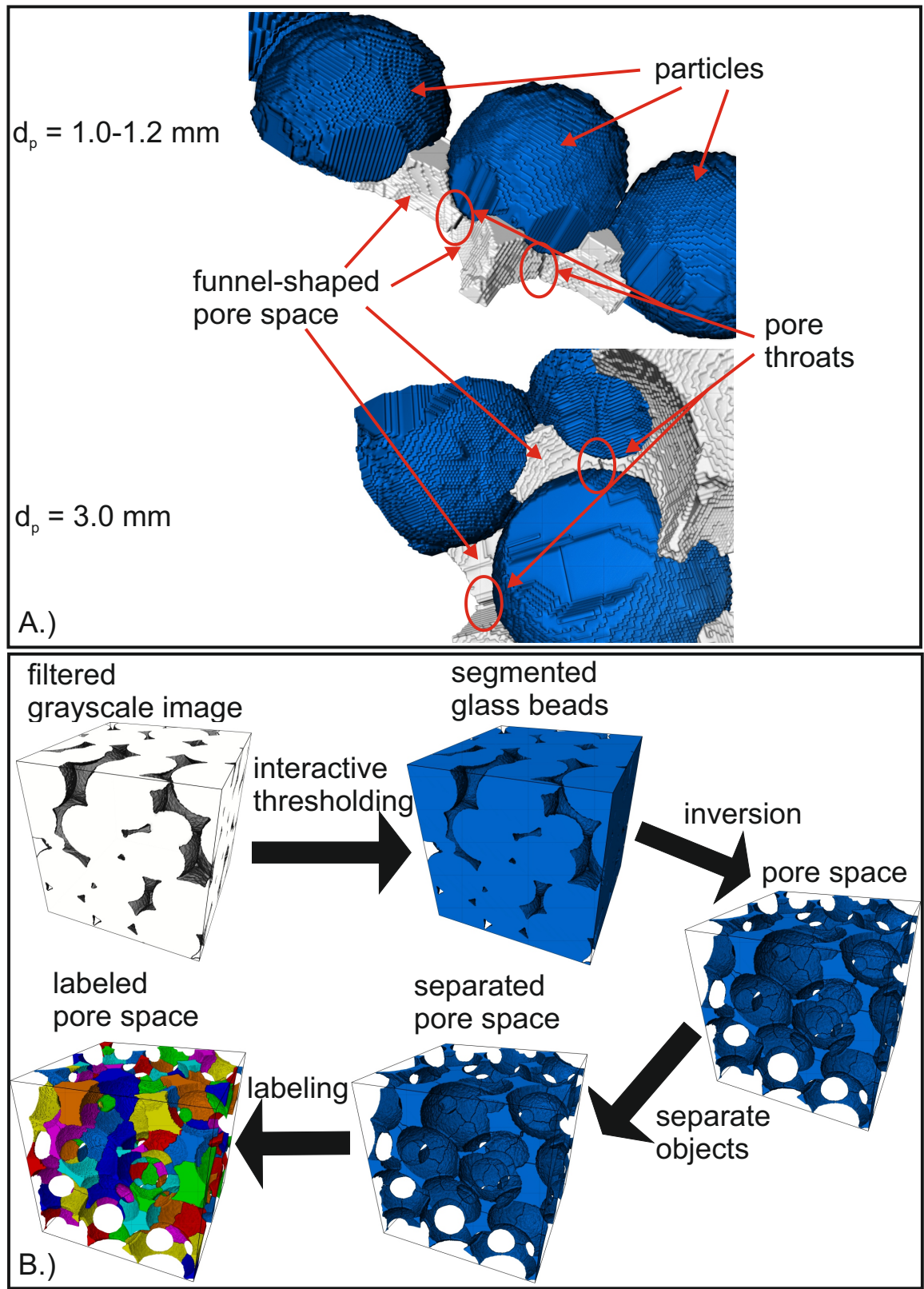

Figure 4.7: A.) Visualization of funnel-shaped pore spaces with distinct pore throats through the examples of glass beads showing particle diameters of $d_{p}=1.0$ - $1.2 \mathrm{~mm}$ and $d_{p}=3.0 \mathrm{~mm}$ after sintering procedure. The pore throats highly influence the coupling between the fluid and solid. B.) Illustration of main image processing steps for visualization and quantification of pore spaces as a result of sintering. 


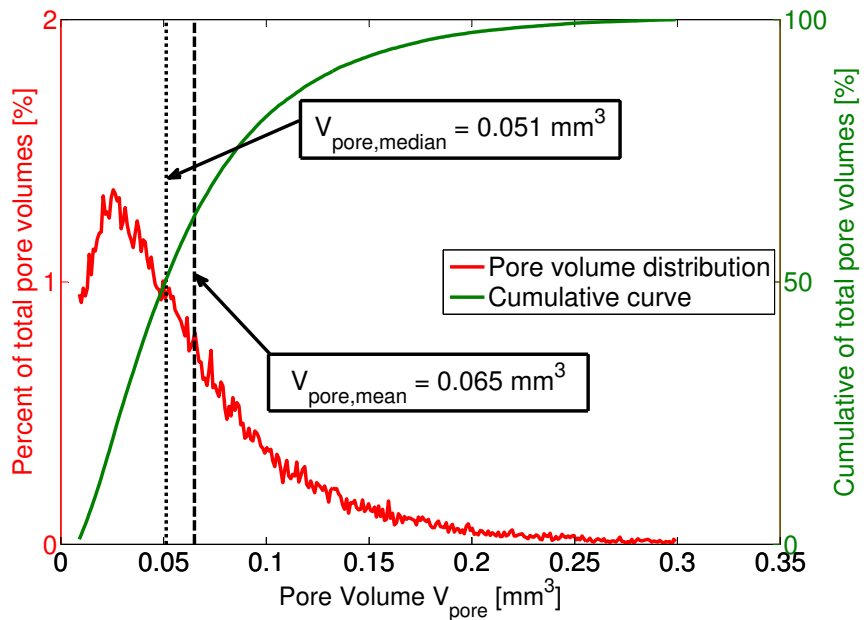

A.)

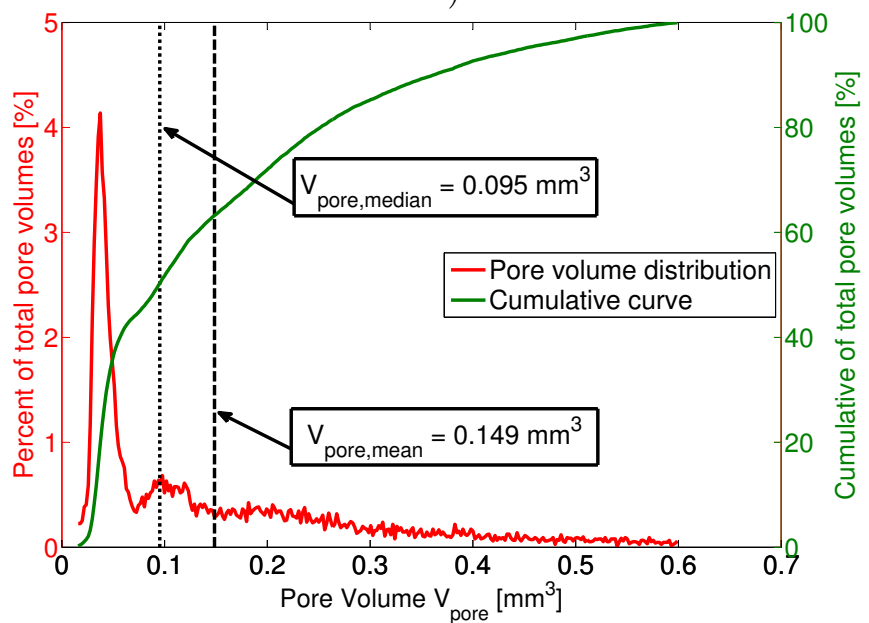

B.)

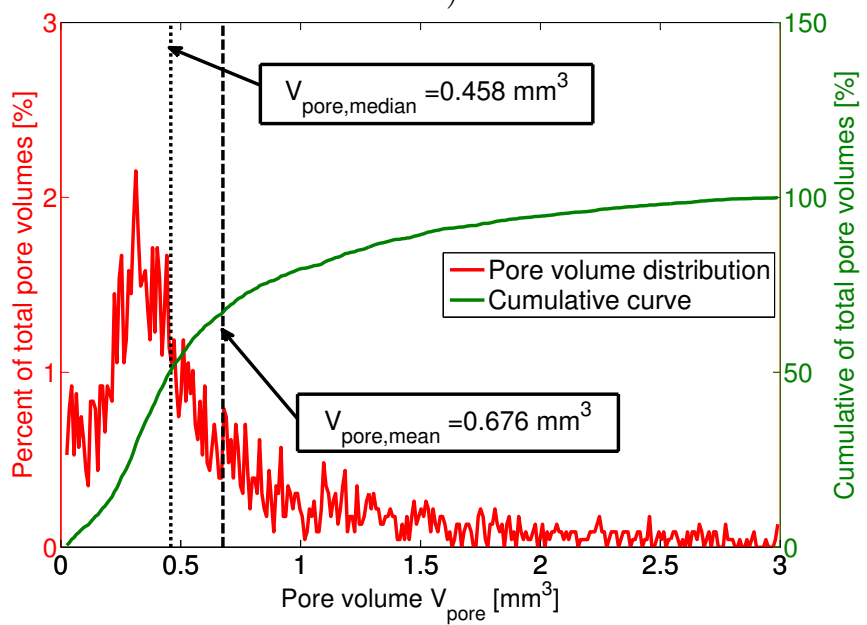

C.)

Figure 4.8: Pore volume $V_{\text {pore }}$ distribution and corresponding cumulative curves of the investigated samples showing different particle diameter: A.) $d_{p}=0.4$ $0.6 \mathrm{~mm} \mathrm{B.)} d_{p}=1.0-1.2 \mathrm{~mm} \mathrm{C.)} d_{p}=3.0 \mathrm{~mm}$ 
Table 4.1: Physical parameters of sintered glass beads and water used in Biot model.

\begin{tabular}{|c|c|c|c|c|}
\hline Parameter & Unit & Sample 1 & Sample 2 & Sample 3 \\
\hline Glass bead diameter ${ }^{[A]} d_{p}$ & {$[\mathrm{~mm}]$} & $0.4-0.6$ & $1.0-1.2$ & 3.0 \\
\hline Density of beads ${ }^{[A]} \rho_{s}$ & {$\left[\mathrm{~g} / \mathrm{cm}^{3}\right]$} & 2.5 & 2.5 & 2.5 \\
\hline Poisson ratio of beads ${ }^{[A]} \nu_{s}$ & {$[-]$} & 0.23 & 0.23 & 0.23 \\
\hline E-modulus of beads ${ }^{[A]} E_{s}$ & {$[\mathrm{GPa}]$} & 59.0 & 59.0 & 59.0 \\
\hline Sinter duration ${ }^{[B]} t_{s d}$ & {$[\mathrm{~h}]$} & 4.5 & 5.0 & 6.0 \\
\hline Number of particles ${ }^{[C]} n$ & {$[-]$} & 87,899 & 8,816 & 1,234 \\
\hline Tortuosity factor ${ }^{[C]} \alpha$ & {$[-]$} & 1.46 & 1.54 & 1.86 \\
\hline Porosity $^{[C]} \phi$ & {$[\%]$} & 37.82 & 32.53 & 20.04 \\
\hline Permeability ${ }^{[D]} k_{z}^{s}$ & {$\left[\mathrm{~m}^{2}\right]$} & $9.16 \times 10^{-11}$ & $9.52 \times 10^{-10}$ & $9.67 \times 10^{-10}$ \\
\hline Time Of Flight ${ }^{[E]} T O F_{f r}$ & {$[\mu \mathrm{s}]$} & 23.52 & 20.57 & 13.51 \\
\hline $\begin{array}{l}\text { E-modulus of solid frame } \\
E_{f r}\end{array}$ & {$[\mathrm{GPa}]$} & 7.0 & 10.0 & 27.3 \\
\hline K-modulus of solid frame $K_{f r}$ & {$[\mathrm{GPa}]$} & 6.9 & 9.8 & 59.0 \\
\hline Rollover frequency $\omega_{r}$ & {$[1 / \mathrm{s}]$} & 283 & 222 & 112 \\
\hline Temperature of fluid $T^{f}$ & {$\left[{ }^{\circ} \mathrm{C}\right]$} & 22 & & \\
\hline Density of fluid $\rho^{f R}$ & {$\left[\mathrm{~g} / \mathrm{cm}^{3}\right]$} & 1 & & \\
\hline K-modulus of fluid $K^{f}$ & {$[\mathrm{GPa}]$} & 2.2 & & \\
\hline
\end{tabular}

$\overline{\overline{[A]}}$ : Manufacturer information

${ }^{[B]}$ : The sinter durations refer to the time period between switching on and off the furnace

${ }^{[C]}$ : Obtained from 1024 x 1024 x 2048 cuboids extracted from $\mu$ XRCT data

$[D]$ : Measured averaged values from 5 or 8 independent permeability experiments

${ }^{[E]}$ : Determined by dry ultrasound measurement 

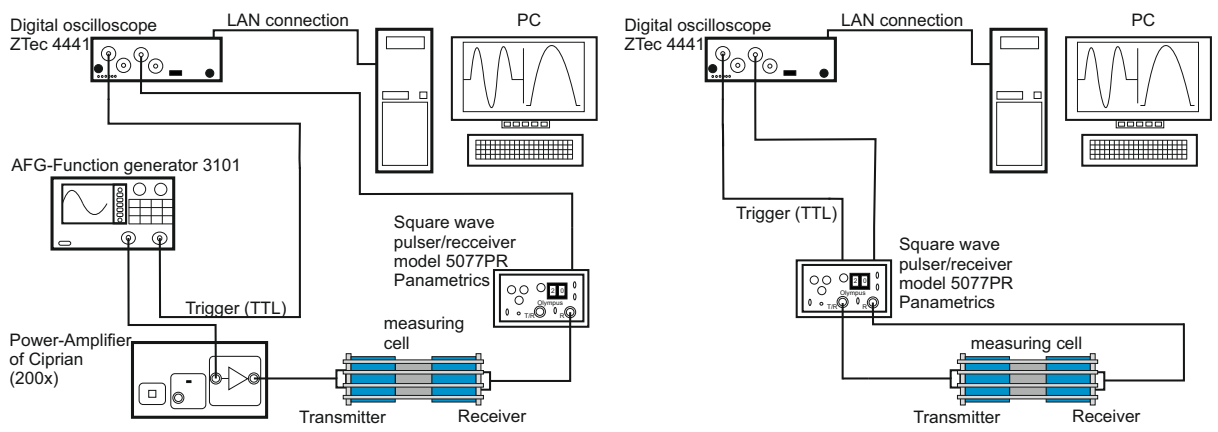

Figure 4.9: Experimental arrangements according to the transmission method, whereby the square wave pulser of Olympus (model 5077PR) is used as pulser and pre-amplifier (left) or simply as pre-amplifier (right).

In configuration 1, depicted in Figure 4.9 (left), a Panametrics 5077PR pulser/ receiver (Olympus) is used to stimulate the ultrasound transducers. Since no further devices are used in this configuration, the system becomes more robust and less prone to errors. A further advantage of this configuration is the absence of so-called side loops in the received signals due to sudden excitation of piezo-electric transducers with high energy, which considerably simplifies the determination of TOF of the received signals. Side loops in received time signals especially occur, when the sound wave propagates through a complex porous medium, such as sintered glass beads, cf. Figure 4.10.

In Figure 4.10 the received time signals of the same water-saturated sample with $d_{p}=1.0-1.2 \mathrm{~mm}$ at different excitation signals are depicted. In the case of sinusoidal excitation the received time signal shows a side loop at the leading edge, while in the case of square wave excitation the side loop disappears. The deviation from the baseline occurs abruptly in a clear manner, which significantly facilitate the determination of the TOF.

In configuration 2, shown in Figure 4.9 (right), the piezo-electric transmitter is excited using an arbitrary function generator (Tektronix model AFG 3101). This configuration enables the selective and different stimulation of the broadband transducers with desired functions at specific frequencies.

For the excitation, either a sinus burst signal with 1 and 10 cycles or a Ricker wavelet with 1 cycle, also known as Mexican hat due to it's shape, are used (see Figure 4.11).

The main advantage of sinusoidal signal is that the major frequency of the generated wave can be uniquely adjusted compared to a Ricker wavelet. However, 

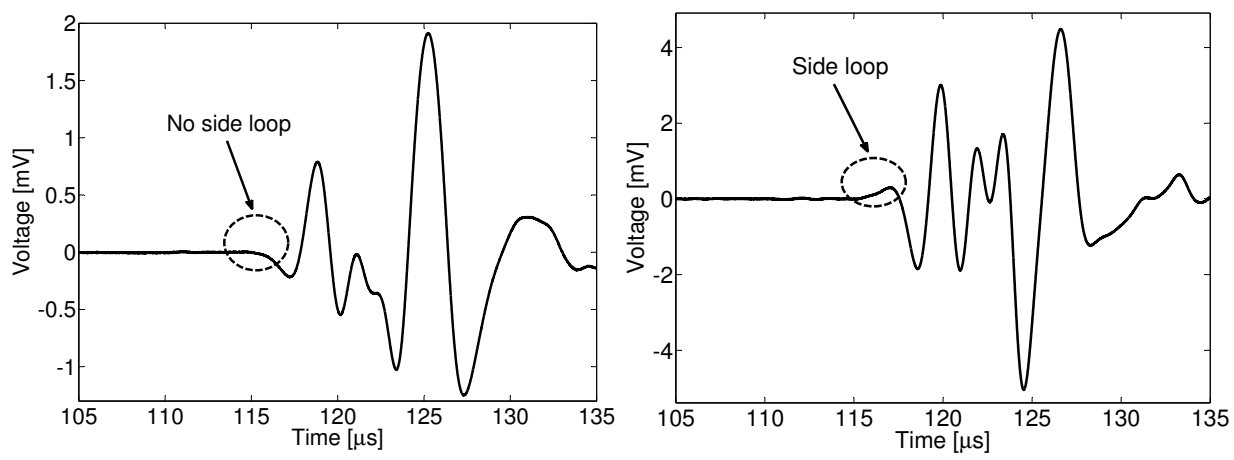

Figure 4.10: Received time signals, after pre-amplifying with $50 \mathrm{~dB}$, transmitted through the same sample with $d_{p}=1.0-1.2 \mathrm{~mm}$. The piezo-electric transducer is excited with a square wave (left) according to configuration 1 and 1-cycle sinus-burst-signal (right) according to configuration 2 in Figure 4.9. The central frequency of the generated signal is at $1.0 \mathrm{MHz}$.

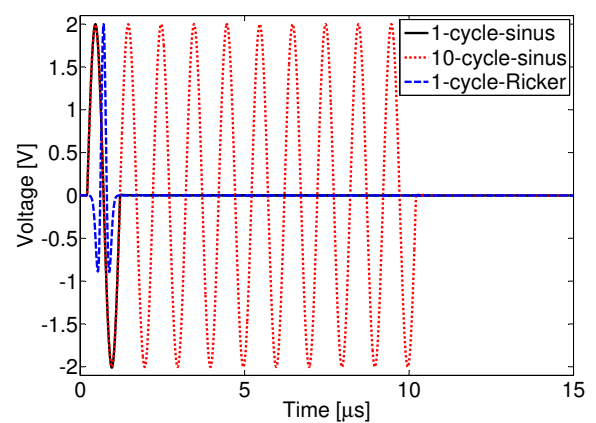

A.)

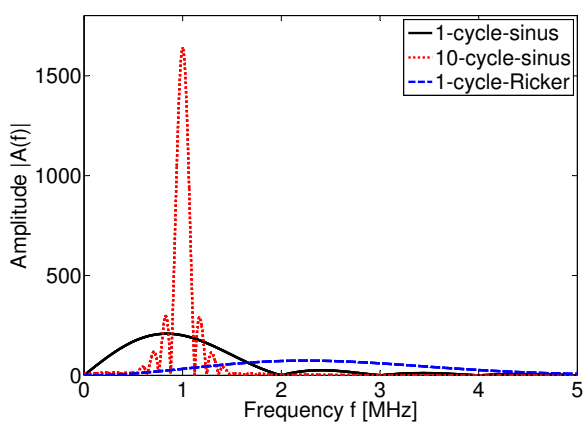

B.)

Figure 4.11: Excitation signals (A.) with corresponding amplitude spectra (B.) at $1 \mathrm{MHz}$ by using configuration 2 in Figure 4.9 (left). 
the sinusoidal signal is not as smooth in the domain as the Ricker wavelet and thus contains "parasitic" frequencies. The Ricker wavelet is a relatively simple seismic model wavelet, since it is the second derivative of a Gaussian function. It is often used to approximate seismic spectra [63]. The main advantage of the Ricker wavelet lies in a broader frequency spectrum compared to a sinusoidal wave with the same duration. A Ricker wavelet with a duration time of $1 \mu \mathrm{s}$ exhibits a frequency spectrum up to $5 \mathrm{MHz}$, while the frequency spectrum of a sinusoidal wave with the same duration is limited to $2 \mathrm{MHz}$, cf. Figure 4.11. One can imagine that a Ricker wavelet with a broader frequency spectrum than a sinus-wave travelling through the fluid-saturated sintered, porous glass bead sample would be more sensitive and capture more detail and thus have a better resolution. Furthermore, Figure 4.11 demonstrates that the frequency spectrum of the polychromatic sinusoidal signal with 1 cycle becomes significantly narrower and the peak significantly increases, when the cycle number is increased to 10 . In this way, the frequency of the generated wave is defined more clearly but in ultrasound experiments short-time signals are preferred due to their localization in space and time. The energy of the wave signal, which is represented by the integral of the amplitude spectra, remains constant, when the cycle number is increased.

\subsubsection{Experimental procedure}

The samples are examined in a newly developed multi-purpose measuring cell, using two pairs of immersion broadband transducers (Panametrics type V303SU, V306-SU, Olympus, Panametrics-NDT) with central frequencies of either 1 or $2.25 \mathrm{MHz}$. Figure 4.12 shows the multi-purpose measuring cell in the mode for ultrasound measurements.

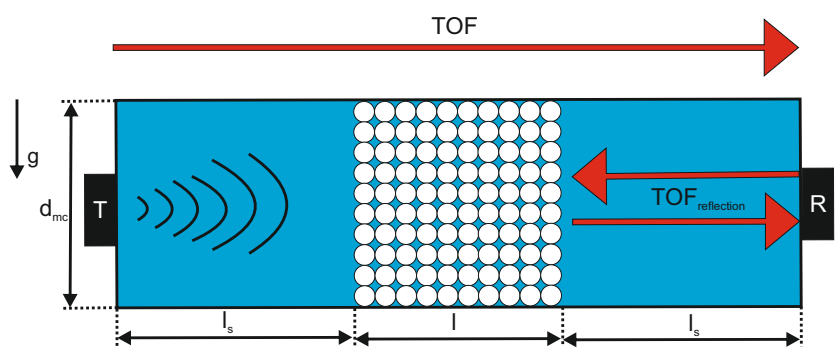

Figure 4.12: Simplified sketch of the measuring cell with length specifications used for ultrasound measurements. 
The cylindrical specimen is pneumatically fixed by a custom made specimen holder. The cover and base are equipped with immersion broadband transducers. A detailed description of the used multipurpose measuring cell is described in [69]. The two 0.5-inch-diameter transducers are mounted in cover and base of the measuring cell, which ensures the plan-parallel alignment of the transmitter and receiver and thus a proper ultrasonic sounding of the porous sample. The distance between the two transducers is fixed at $196 \mathrm{~mm}$. The distance between the transducers and the porous sample is fixed at $l_{s}=73 \mathrm{~mm}$. In this way, the sample is not exposed to the near field of the transmitter. The arrival time of the back reflected signal can be precisely determined from the distance of the receiver to the sample and the measured speed of sound in water.

The measuring cell including the porous sample is rinsed with carbon dioxide prior to the saturation with de-aired and filtered water and the actual ultrasound experiments. In this way, the content of air bubbles in the measuring cell could be minimized and an optimal saturation of the porous sample with water could be achieved.

The received time signals are digitized using an oscilloscope (ZTec 4441 model) showing a vertical resolution of 14 bit and are finally displayed and stored on computer via LAN-connection. A fast Fourier transformation (FFT) is performed to determine the power spectrum of the transmitted signal.

\subsubsection{Spectral Ratio Technique (SRT)}

For the determination of (frequency-dependent) velocity and attenuation effects of the water-saturated sample according to SRT, the amplitude spectrum is compared to the spectrum of the reference measurement in water without placing a porous sample between the transducers, cf. Figure 4.13 A.-B.). On basis of Figure 4.13 A.), the bulk-velocity in the time domain within the porous samples can be computed as

$$
c=\frac{1}{\frac{1}{c_{\mathrm{ref}}}+\frac{\Delta T O F}{l}},
$$

whereby $c_{\text {ref }}$ is the speed of sound in water and $l$ the sample length of the investigated sintered porous sample. The speed of sound in water is examined from the TOF at approximately $1485 \mathrm{~m} / \mathrm{s}$ using the experimental arrangement shown in Figure 4.9 (left). $\triangle T O F=T O F-T O F_{\text {ref }}$ is the time difference between the sample and water pulses.

However, the transmitted ultrasound wave changes its shape and frequency content especially while propagating through a sintered glass bead sample, the time difference is difficult to determine in the time domain. Therefore the phase spectrum method is used $[91,152]$ instead of the determination in the time domain. Here, the velocity is determined in the frequency domain after applying a FFT 
to the sample and water impulses. In this method, the time difference can be expressed through the phase difference as

$$
\Delta T O F(\omega)=\frac{\Delta \phi(\omega)}{\omega},
$$

with $\Delta \phi=\phi-\phi_{\text {ref }}$ and $\omega=2 \pi f$ as the angular frequency. The phase difference results from the imaginary and real parts of the amplitude spectra obtained from the signals after FFT as follows

$$
\phi=\arctan \left(\frac{\operatorname{Im}[A(\omega)]}{\operatorname{Re}[A(\omega)]}\right) \quad \phi_{\mathrm{ref}}=\arctan \left(\frac{\operatorname{Im}\left[A_{\mathrm{ref}}(\omega)\right]}{\operatorname{Re}\left[A_{\mathrm{ref}}(\omega)\right]}\right) .
$$

However, the arctan-function provides only phase values within a range of $-\pi / 2$ and $+\pi / 2$, termed as wrapped phase. For a continuous phase the spectrum is unwrapped into its true form by adding or subtracting multiples of $2 \pi$ to the principal value until the phase jumps induced by modulo of $2 \pi$ are removed $[107,131]$. The so-called unwrapped phase can be defined as

$$
\phi_{u}(\omega)=\phi(\omega) \pm 2 \bar{k} \pi
$$

where $\bar{k}$ is an integer. Substitution of Equation (4.40) and Equation (4.41) in Equation (4.38) leads to the frequency-dependent phase velocity

$$
c(\omega)=\frac{1}{\frac{1}{c_{\mathrm{ref}}}+\frac{\Delta \phi_{u}}{\omega l}} .
$$

Equalizing the Equations (4.25), (4.31) and (4.38) for the elimination of the velocities and resolving for TOF results in frequency-dependent arrival times

$$
\begin{array}{r}
T O F_{P 1, P 2}(\omega)=T O F_{\text {ref }}-\frac{l}{c_{\mathrm{ref}}}+\frac{l\left|\operatorname{Re}\left[\sqrt{\zeta_{P 1, P 2}(\omega)}\right]\right|}{\omega}, \\
\operatorname{TOF}_{S}(\omega)=T O F_{\mathrm{ref}}+l\left(\frac{1}{c_{\mathrm{ref}}}+\frac{\operatorname{Re}\left[\sqrt{\zeta_{S}(\omega)}\right]}{\omega}\right)
\end{array}
$$

for the different wave types. Equations (4.43) and (4.44) link the time domain with the frequency domain and can be used to predict the arrival times and frequency content of the different wave types using the solid and fluid properties listed in Table 4.1. Using Equations (6.6) and (4.44) has the advantage that the TOF can be directly determined from ultrasound measurements and compared with predicted values, in order to identify the detected waves. The apparent frequency-dependent attenuation, that describes the signal loss within the dis- 


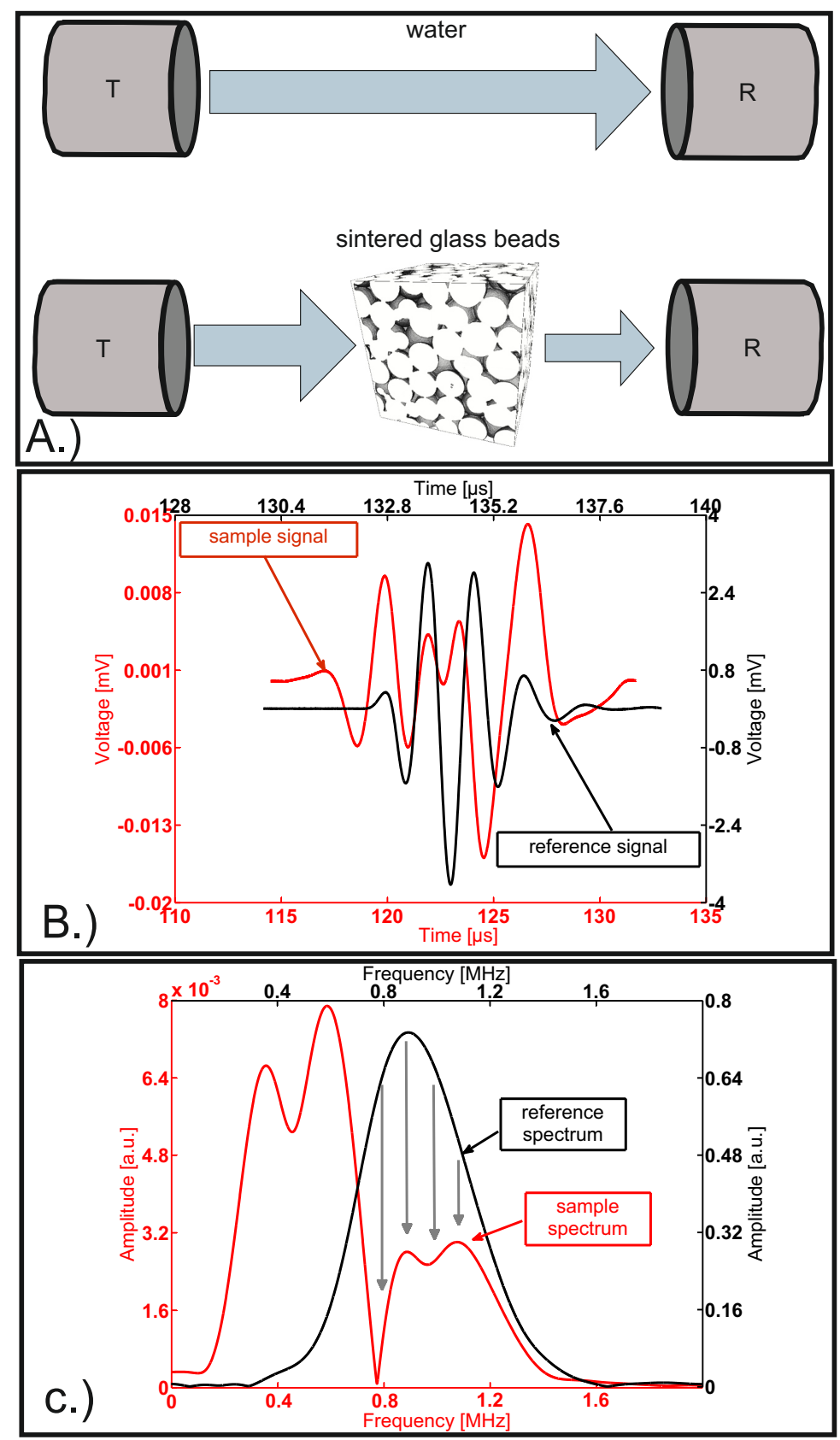

Figure 4.13: Illustration of principles of Spectral Ratio Technique (SRT): A.) Substitution principle through the use of sintered glass beads B.) Examples of received ultrasound signals recorded in reference medium, such as water (black line) and through water-saturated sintered glass bead sample (red line). C.) Amplitude spectra from the reference signal (black line) and of of the signal transmitted through the water-saturated sample (red line). From the spectral ratio of both signals, the frequency-dependent specific attenuation and phase velocities can be determined. 
persive sample, can be directly determined from the amplitude spectra ratio of sample and water measurement and is defined on a logarithmic scale as

$$
\alpha(f)=\hat{\alpha}(f) l=\ln \frac{\left|A_{\mathrm{ref}}(f)\right|}{|A(f)|},
$$

where $\hat{\alpha}$ is the normalized attenuation per sample length $l$. For a better comparison between the samples with different length, the normalized attenuation $\hat{\alpha}$ are often used. The specific attenuation $1 / Q$ can be estimated from the slope of the apparent attenuation $\alpha$. Taking into account the definitions in Equations (4.25) and (4.31), the specific attenuation $1 / Q$ can be alternatively expressed in terms of the attenuation $\hat{\alpha}(f)$ and phase velocity $c(f)[125]$ as

$$
\frac{1}{Q}=\frac{\hat{\alpha}(f) c(f)}{\pi f}
$$

\subsection{Results and discussion}

In this section, we first demonstrate the theoretical predictions according to Biot's theory using parameters for the solid and fluid according to Table 4.1. In the following section, the results from the ultrasound experiments are presented, described and analyzed in terms of time signals, TOFs, attenuation, phase velocities, amplitude spectra and spectrograms.

\subsubsection{Theoretical investigations}

Figure 4.14 shows the theoretically predicted curves for the frequency-dependent Time Of Flights (A. - C.) and specific attenuations (D. - F.) of the different wave types obtained from dispersion relations described in section 4.2.2 and 4.2.3. The frequency-dependent TOFs are determined according to Equations (4.43) and (4.44) using the solid and fluid parameters in Table 4.1. The vertical red lines denote the critical frequency from Equation (4.1), which separates low- and high-frequency regimes described in section 4.2. Depending on the properties of the solid skeleton, such as tortuosity, porosity or intrinsic permeability, the determined critical frequencies of the investigated samples in water range only between 112 and $222 \mathrm{~Hz}$, which confirm that the performed ultrasound experiments have taken place in the high-frequency regime, where theoretically both fast and slow p-wave can propagate.

The gray-shaded areas in Figure 4.14 A.-F.) represent the frequency regime of the coherent parts of the detected signals, where dispersion effects can be excluded. The predicted frequency-dependent TOFs of the different wave types are 


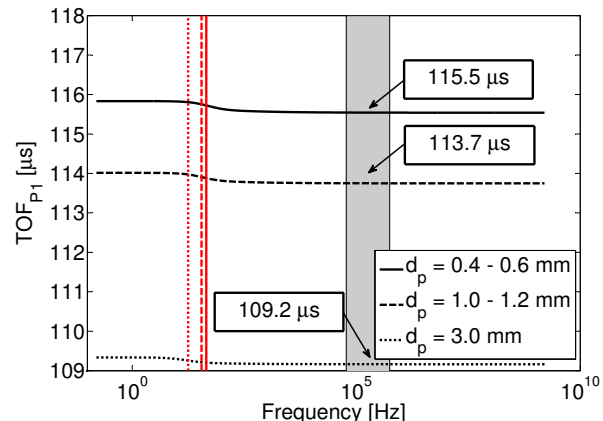

A.)

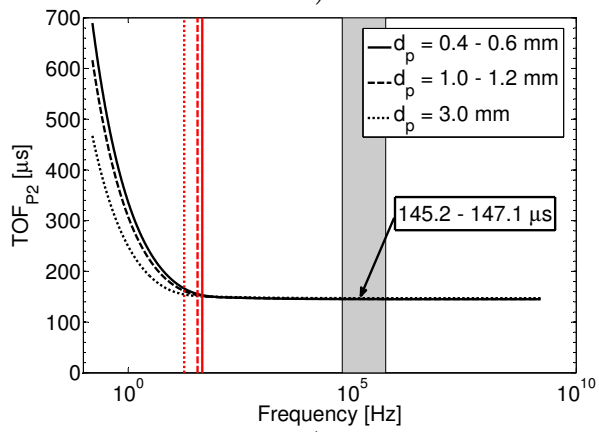

C.)

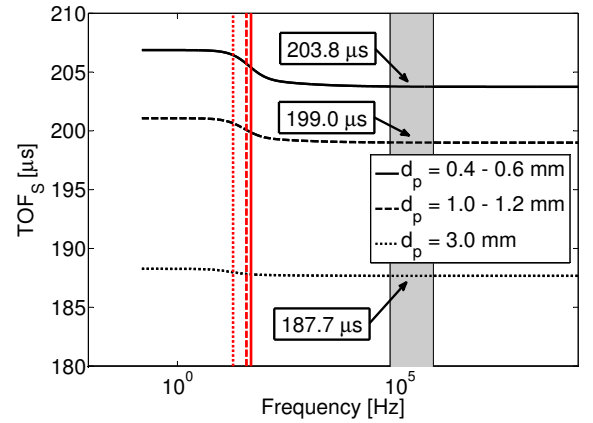

E.)

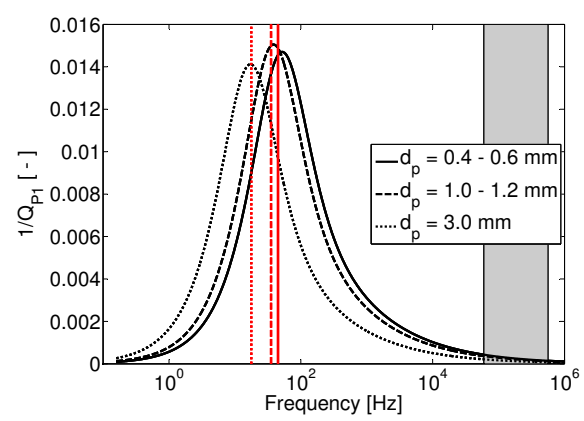

B.)

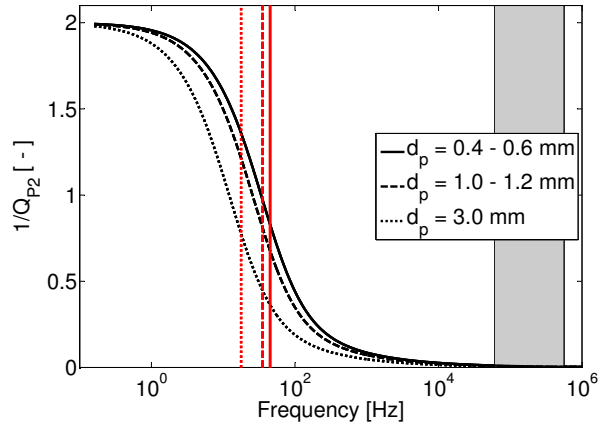

D.)

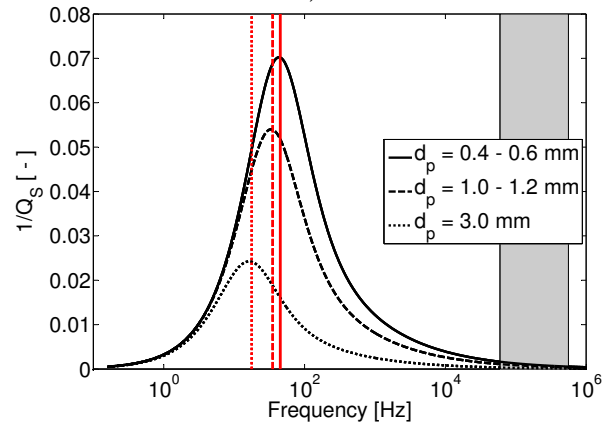

F.)

Figure 4.14: Illustration of the frequency-dependent TOFs and specific attenuations of the different waves for the investigated water-saturated sintered samples: The left panel shows the frequency-dependent TOFs (A., C., E.) and the right panel the specific attenuations (B., D., F.) of the different waves. The theoretical predicted curves are determined from dispersion relations formulated in Biot theory using the physical parameters in Table 4.1 . 
inversely proportional to their velocities and remain constant in the relevant frequency range of the detected coherent pulse (gray-shaded area) independently of the wave type and investigated sample. The difference in the arrival times of the fast P1- and shear-wave between the investigated samples are very large, whereas the theoretically predicted arrival times of slow P2-wave of the investigated samples do not differ considerably from each other in the high-frequency regime. In the high-frequency regime the TOFs for the slow P2-wave of the investigated samples range between 145 and $147 \mu \mathrm{s}$. For $\omega \rightarrow \infty$, the TOFs of the different samples converge to a final value and remain constant in their high-frequency limits.

The specific attenuations $Q^{-1}$, also known as inverse quality factor, of the fast P1- and the shear-wave show distinct peaks around the critical frequencies of the investigated samples. The decoupling process between the two constituents induces a viscous relative movement of fluid and solid, which results in a momentum loss for the P1-wave at the critical frequency. This damping mechanism caused by viscous drags is known as the Biot-like damping [24, 25, 105]. For decreasing porosities of the investigated samples, the specific attenuation results in a shift of the $Q^{-1}$ peaks to higher frequencies. The specific attenuation of the slow $\mathrm{P} 2$-wave equals two in the low-frequency because it is not propagating due to strong viscous coupling. With increasing frequency, when inertia effects become dominant and the fluid starts to decouple, the specific attenuation of the slow Biot-wave decreases strongly [105]. The decoupling of fluid and solid phases and thus the transitions to the high-frequency regime tends to occur earlier for the sample with particle diameters of $3.0 \mathrm{~mm}$, i.e. at lower frequencies, due to the higher permeability of the sample.

\subsubsection{Experimental observations}

\section{Time Signals}

For the performed ultrasound experiments a time period of $500 \mu \mathrm{s}$ is recorded and finally analyzed. Figures 4.15 A.) to C.) illustrate exemplary the time signals of the investigated samples and the reference measurement in water (D.), whereby a 10-cycle-sinus-burst-signal with frequency of $0.9 \mathrm{MHz}$ is used as excitation signal. As denoted in Figure 4.15, one can see that the received signals consist basically of a coherent deterministic pulse and an incoherent part composed of (multiple) scattered waves. The intensity of multiply-scattered waves is increasing (in relation to the intensity of coherent impulse) with increasing particle diameter of the glass beads, whereas the amplitude of the coherent pulse decreases. In the case of the sintered glass bead packing with particle diameters of $3.0 \mathrm{~mm}$, the intensity of the coherent deterministic pulse is lower than the intensity of the incoherent part caused by scattering effects compared to the samples with 
smaller diameters.

In order to demonstrate that the received signals not only depend on the sample but also on the frequency of the excitation signals, Figure 4.16 illustrates the temporal signal of the water-saturated sintered porous sample showing particle diameters between 1.0 and $1.2 \mathrm{~mm}$ at two different frequencies. In the case of the time signal, depicted in Figure 4.16 (A.) the piezo-electric acoustic transmitter is excited with a 10-cycle-sinus-burst-signal at frequency of $0.2 \mathrm{MHz}$, whereas in the case of the time signal, shown in Figure 4.16 (B.) the dominant frequency of the generated wave is at $1.0 \mathrm{MHz}$.

The transmitted temporal signal basically consists of a primary low-frequency coherent pulse, which arrives at the leading edge and is characterized by large amplitudes and a high-frequency incoherent part mainly composed of (multiply-) scattered and reflected waves. However, the incoherent part of the generated highfrequency wave with $1.0 \mathrm{MHz}$ shows speckle-like scattered waves, which are highly sensitive to the underlying microstructure and caused by the inhomogeneous distribution of the contact network and the force chains of the glass beads [92, 137,139 ], whereas the incoherent part of the low-frequency wave (at $0.2 \mathrm{MHz}$ ) is more of macroscopic nature.

In the following we focus on the coherent deterministic pulse, which arrives at certain earlier times. We will confirm that the coherent part, which describes the macroscopic properties of the saturated porous bulk medium, is well-described by the established Biot theory.

So far, it is noticeable, that the low-frequency wave of $0.2 \mathrm{MHz}$, composed of 10 sinus cycles, is able to easily pass through the sintered porous sample without capturing the complex structure, whereas the coherent pulse of the high-frequency wave with $1.0 \mathrm{MHz}$ arrives completely distorted. The comparison of the power spectra of the coherent part of the low- and high-frequency wave in the following section confirms the distortion of the high-frequency wave, cf. Figure 4.17 (C.).

\section{Windowing and amplitude spectra}

In order to separate the coherent part from the remaining part of the raw signal, temporal windows are used. As an example for the windowing procedure Figure 4.17 (A.-B) shows the received untreated raw time signal (black line) and the windowed time signal (red line) after applying a so-called "Tukey"-window on the coherent pulse. The "Tukey"-window can be regarded as tapered cosine window [34]. Note that the input parameter was chosen at $\alpha_{\text {Tukey }}=0.1$ in order to reduce the occurrence of side lobes, which are caused by the so-called leakage effect and thus not of physical nature. $\alpha_{\text {Tukey }}=0$ corresponds to a rectangular window and $\alpha_{\text {Tukey }}=1$ to a "Hann"-window [34]. 


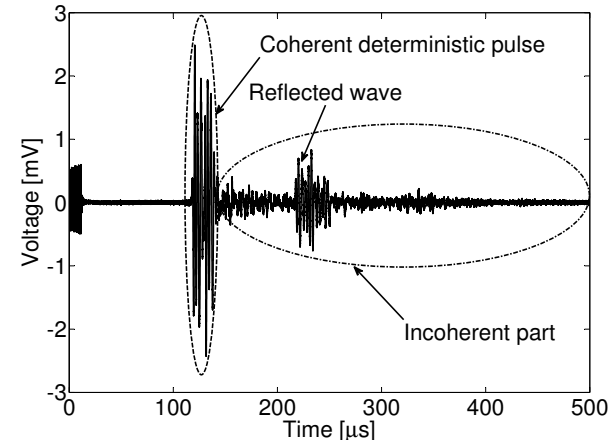

A.)

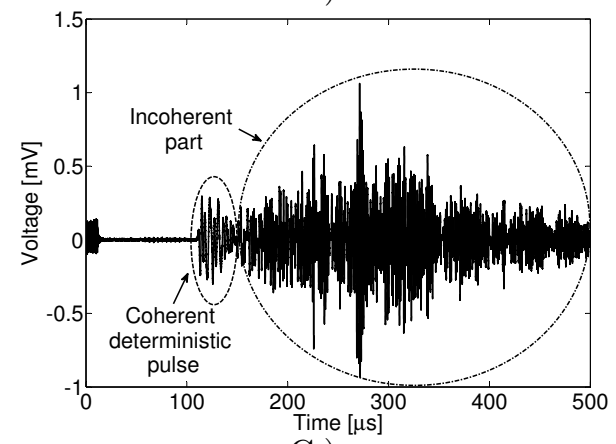

C.)

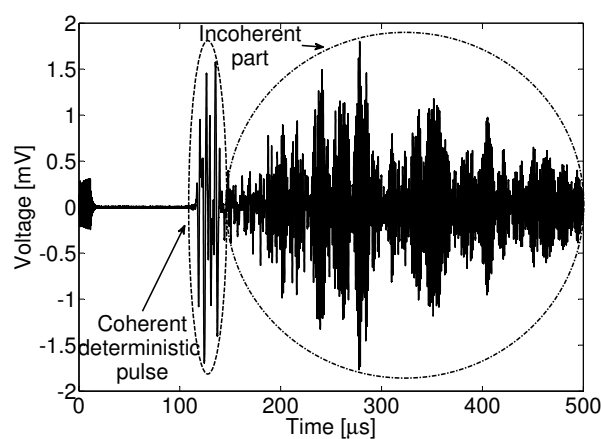

B.)

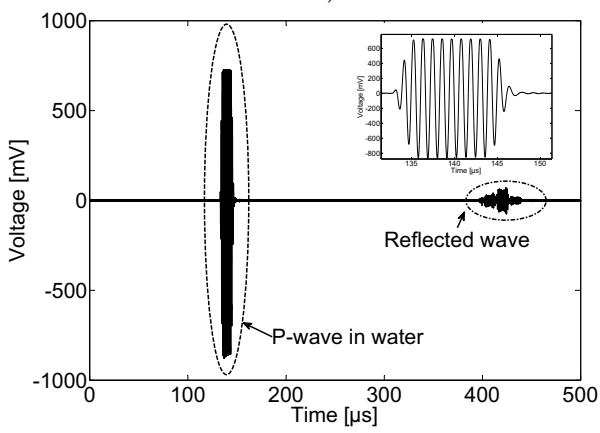

D.)

Figure 4.15: Received time signals through water-saturated samples with different glass bead diameters and in water: A.) $d_{p}=0.4-0.6 \mathrm{~mm} \mathrm{B.)} d_{p}=1.0-1.2 \mathrm{~mm}$ C.) $d_{p}=3.0 \mathrm{~mm}$ D.) reference measurement in water. In each measurement, the acoustic transmitter is excited with a 10-cycle-sinus-burst signal at $0.9 \mathrm{MHz}$. The inset at stage D.) shows a zoom of the reference signal in water consisting of 10 sinus cycles. 


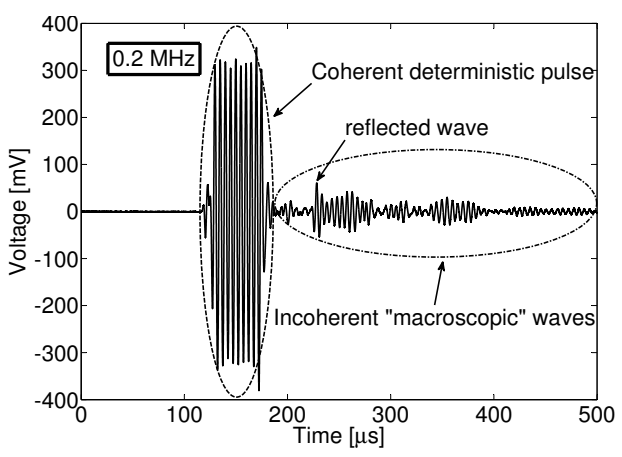

A.)

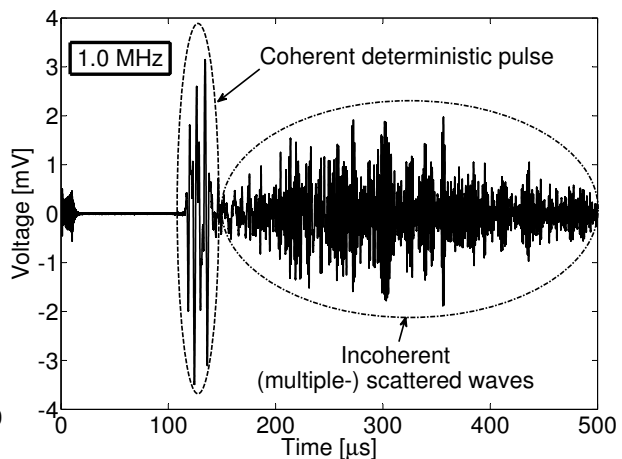

B.)

Figure 4.16: Received transmission signals through a water-saturated sintered sample showing glass bead diameters between 1.0 and $1.2 \mathrm{~mm}$. The piezo-electric acoustic transmitter is excited with a 10-cycle-sinus-burst signal at different frequencies of $0.2 \mathrm{MHz}$ (A.) and $1.0 \mathrm{MHz}$ (B.).

The acoustic transmitter has been excited with a 10-cycle-sinus-burst-signal at two specific frequencies of 0.2 and $1.0 \mathrm{MHz}$. One can clearly see, that the highfrequency incoherent part is composed of scattered and reflected waves, which arrive certainly later than the coherent pulse, are set to zero after windowing. In the following evaluation of the ultrasound signals only the coherent parts are considered for a Discrete Fast Fourier Transformation (DFFT). It must be noticed, that the temporal window widths for the fast Fourier transformation of the coherent deterministic pulse are chosen in accordance with the time periods of coherent pulses, cf. Figure 4.17.

The normalized power spectra of the generated low-frequency wave at $0.2 \mathrm{MHz}$ obtained from reference and sample measurement, illustrated in black and red in Figure 4.17 (C.), show peaks around $0.2 \mathrm{MHz}$, whereas the higher-frequency components of the reference signal $(f>0.2 \mathrm{MHz})$ are filtered out by the porous sample. This indicates that the low-frequency part of the wave could easily pass through the sample without capturing the complex morphological structure of the sintered sample and thus without being distorted. In contrast, the peaks of the generated high-frequency wave with $1.0 \mathrm{MHz}$, transmitted through the water-saturated sample, (depicted in blue in Figure 4.17 (C.)), is shifted to lower frequencies compared to the reference measurement in water which indicates that the generated high-frequency wave arrives completely distorted after 


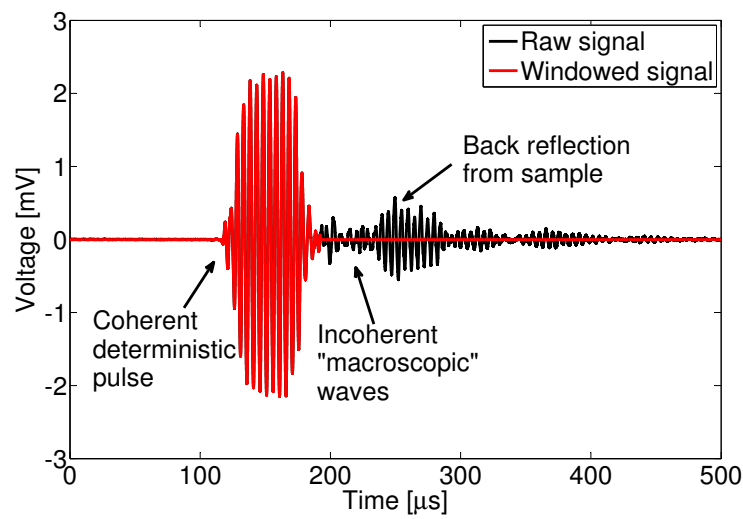

A.)

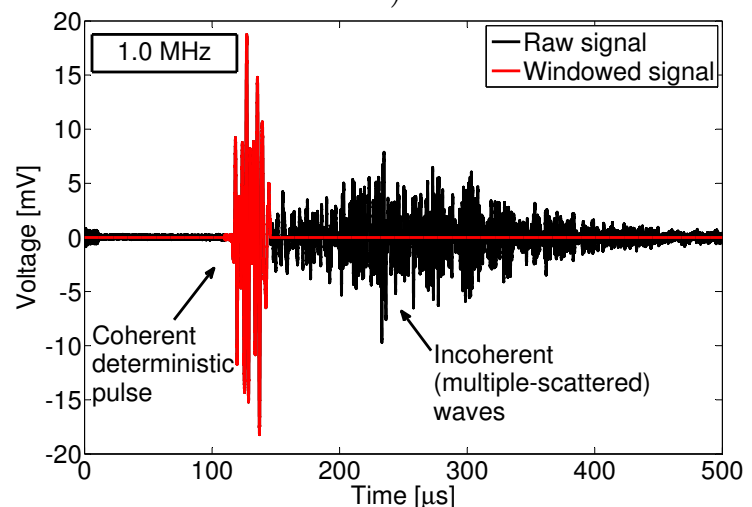

B.)

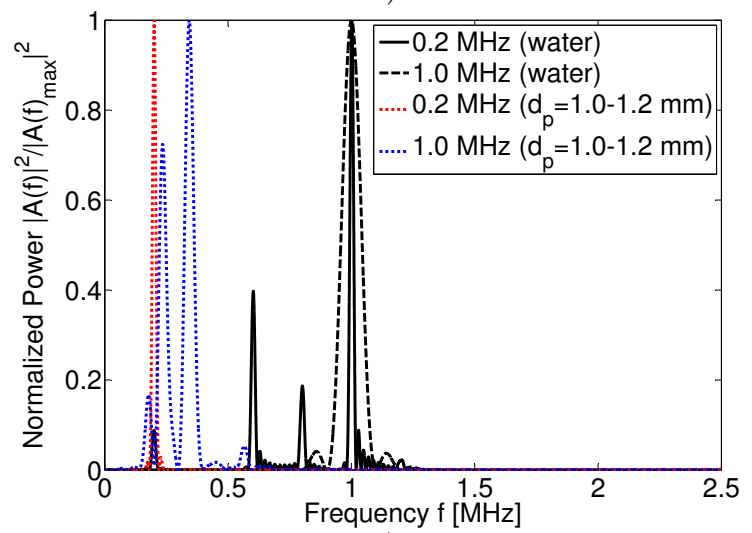

C.)

Figure 4.17: Received and windowed time signals of the generated low- (A.) and high-frequency (B.) wave for the sintered sample with $d_{p}=1.0-1.2 \mathrm{~mm}$ (shown in Figures 4.16 (A.) and (B.)). The power spectra of the coherent parts of the received signals with the corresponding reference measurement in water are shown in C.). A so-called "Tukey"-window is used to separate the coherent part of the signal from the remaining part. 
passing through the sintered glass bead samples. The sample acts as a low-pass filter, which allows the low-frequency parts of the signals to pass through the sample and filters out high-frequency components of the broadband signal. Depending on particle diameter, degree of polydispersity and sintering treatment, the natural cut-off frequency of sintered samples can differ. Figures 4.25 and 4.26 (in the appendix) show the raw and windowed signals with the corresponding power spectra for different excitation signals. In general, the cut-off frequency decreases with increasing polydispersity, particle diameter and sintering duration of the sample. Mouraille et al. [126, 127] and Lawney et al. [108, 109] have investigated the sound propagation in mono- and weakly polydisperse packings numerically with DEM simulations and demonstrated the low-pass filtering effect in granular packings, getting stronger with increasing disorder. In this study similar low-pass filtering behavior has been found in water-saturated sintered glass bead samples, where the cut-off frequency increased with increasing particle diameter and sinter degree.

In order to demonstrate the low-pass filter effect of the investigated sintered samples, Figure 4.18 shows the normalized power spectra, where different input signals at $1 \mathrm{MHz}$ are used. From Figure 4.18 (A.) one observes that the largest peaks of the power spectra are shifted to lower frequencies with increasing particle diameter, when the transmitter is excited with a 1-cycle-sinus-burst-signal at $1 \mathrm{MHz}$. The sintered sample with particle diameter of $3 \mathrm{~mm}$ (and lower porosity due to longer sintering duration) shows, independently of the used excitation signal, the smallest cut-off frequency, and hence the largest filter effect. The frequency and time shift of the coherent parts of the sample signals, in relation to the reference signal in water, are also observed in the spectrograms, where the received signals for 1-cycle-sinus-burst input signal are processed. The spectrograms are calculated from the short-time-Fourier transform by segmenting the coherent part of the signals into short time periods and estimating the spectrum over sliding windows. Figure 4.19 illustrates the Power, respectively as function of time and frequency. The broader frequency spectrum and time period of the reference signal in water, shown in Figure 4.19 (D.), becomes significantly diminished and narrower and the peak is shifted to lower frequencies and earlier arrival times, when a porous sintered glass bead sample is positioned between the transducers. Both frequency and time-shift of the peak, for the porous sample with $d_{p}=3.0 \mathrm{~mm}$, are higher compared to the other two samples, due to the higher stiffness of the porous skeleton and larger particle diameters.

\section{TOF determination}

In Figure 4.20 (A.-C) the time signals for $0.1 \mathrm{MHz}$ input are depicted. The piezo-electric transmitter is stimulated briefly with a square wave impulse using 


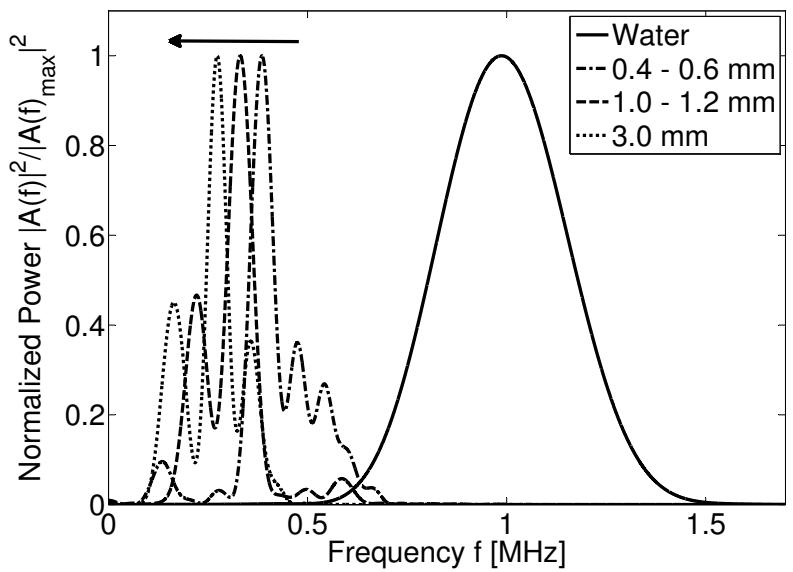

A.)

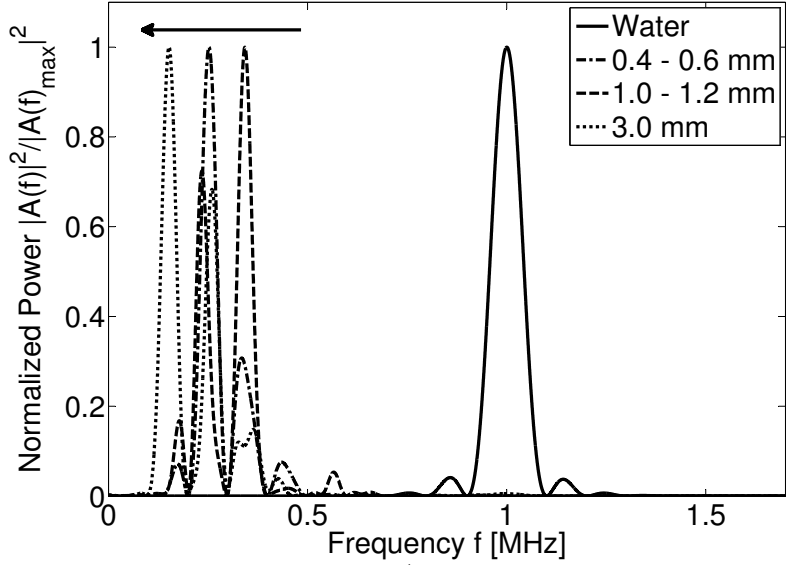

B.)

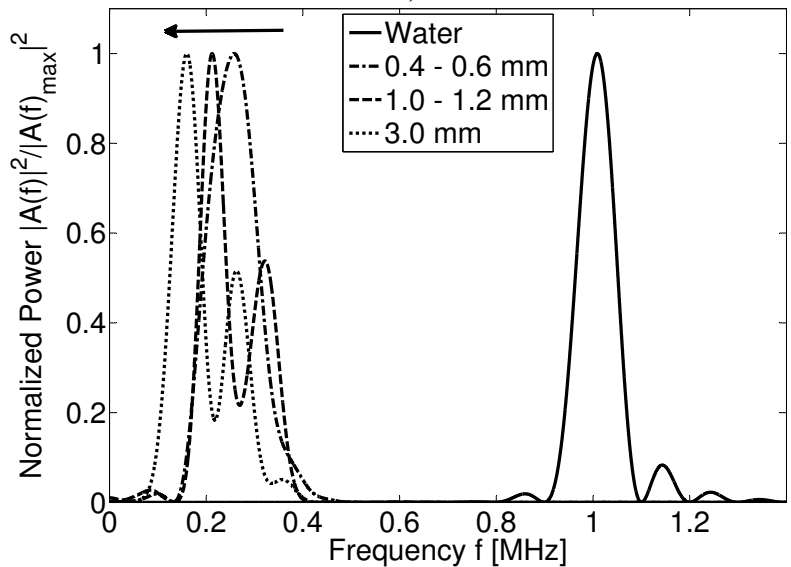

C.)

Figure 4.18: Normalized power spectra of the investigated samples at different high-frequency (1.0 MHz) excitation signals: A.) 1-cycle-sinus-burst B.) 10-cyclesinus-burst C.) 1-cycle-Ricker-wavelet. For comparison, the corresponding normalized power spectrum obtained from reference measurments in water is also shown. The arrow indicate the increasing particle diameter $d_{p}$ for the peaks. 


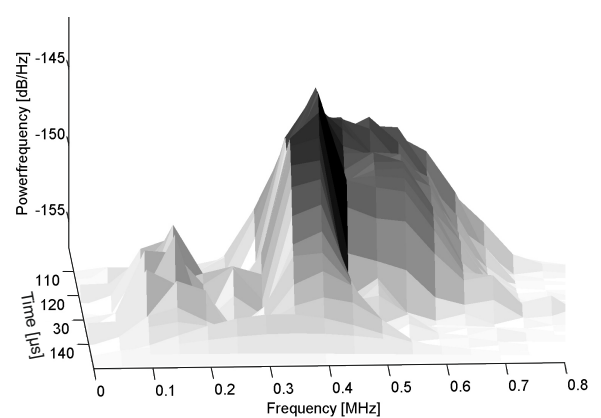

A.)

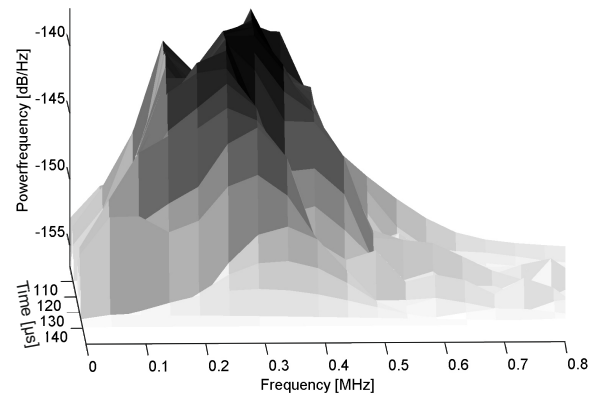

C.)

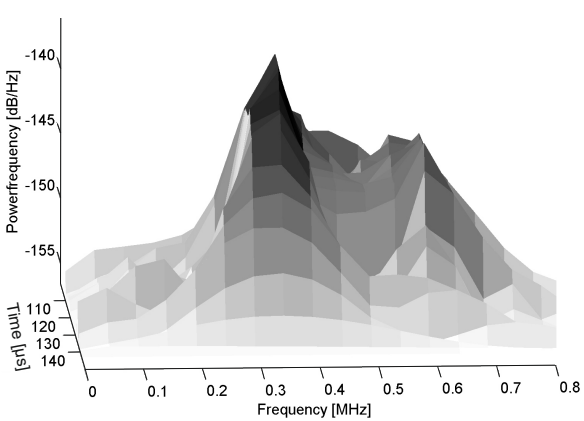

B.)

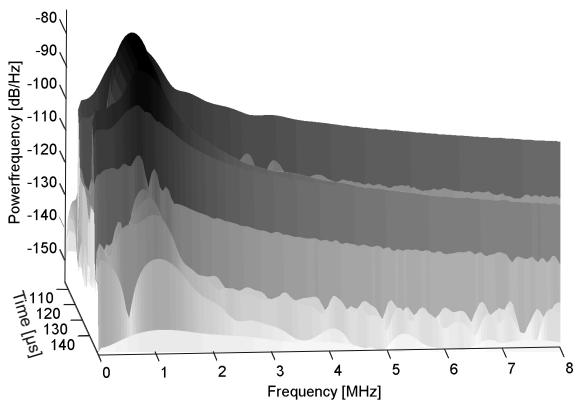

D.)

Figure 4.19: Spectrograms of the different samples and reference measurement, whereby the piezo-electric transmitter is excited with sinus-burst-signal at $1 \mathrm{MHz}$ : A.) $0.4-0.6 \mathrm{~mm}$, B.) $1.0-1.2 \mathrm{~mm}$, C.) $3.0 \mathrm{~mm}$, and the D.) reference measurement (water). Note the different vertical range of for each spectrogram. 
Table 4.2: Experimental and theoretical determined TOFs and SOSs (Speed Of Sound) of fast P1-wave of investigated sintered samples.

\begin{tabular}{lcccc}
\hline Parameter & Unit & Sample 1 & Sample 2 & Sample 3 \\
\hline \hline Particle diameter $d_{p}$ & {$[\mathrm{~mm}]$} & $0.4-0.6$ & $1.0-1.2$ & 3 \\
Time Of Flight $^{[A]}{ }^{2} O F_{\exp }$ & {$[\mu \mathrm{s}]$} & 116.5 & 114.8 & 109.3 \\
Time difference $^{[A]} \Delta T O F_{\text {exp }}$ & {$[\mu \mathrm{s}]$} & -15.8 & -17.5 & -23.0 \\
Time Of Flight $^{[B]}$ TOF $_{\text {theory }}$ & {$[\mu \mathrm{s}]$} & 115.5 & 113.7 & 109.2 \\
Speed Of Sound $^{[B]} c_{\text {theory }}$ & {$[\mathrm{m} / \mathrm{s}]$} & 2594 & 2878 & 4046 \\
Speed Of Sound $^{[A]} c_{\text {exp }}$ & {$[\mathrm{m} / \mathrm{s}]$} & 2784 & 3075 & 4646 \\
\hline \hline
\end{tabular}

$\overline{[A]}$ : Experimentally determined Time Of Flights $\left(T O F_{\exp }\right)$, Time Of Flight differences $\left(\triangle T O F_{\exp }\right)$ related to the reference measurement to water and sound velocities $\left(c_{\exp }\right)$ of fast P1-wave obtained from time domain.

${ }^{[B]}$ : Estimated Time of Flights $\left(T O F_{\text {theory }}\right)$ and sound velocities of fast P1-wave according to Biot's theory.

the experimental arrangement in Figure 4.9 A.). The power spectra of the coherent parts of the received signals are presented in Figure 4.20 D.). The comparison of theoretically and experimentally determined TOFs shows that the detected coherent impulse is the fast P1-wave, which arises from the coupling between the fluid and solid due to inertial effects, cf. Table 4.2. The signals obtained from the sample with particle diameters of $3.0 \mathrm{~mm}$ arrives earlier then the signals obtained from samples with bead diameters of $0.6-0.8 \mathrm{~mm}$ and $1.0-1.2 \mathrm{~mm}$ due to higher stiffness of the solid skeleton as a result of longer sintering duration. Moreover, the higher tortuosity of the pore channels of the $3.0 \mathrm{~mm}$-sample ensures a stronger coupling between solid and fluid phase, which results in a shorter arrival time $(109.3 \mu \mathrm{s})$ of the fast P1-wave.

\section{Phase velocity, attenuation and quality factor}

In Figure 4.21, a comparison between experimentally and analytically determined sound velocities in dependence on the excitation frequency is shown, whereby the acoustic transmitter is excited with input signals. The phase velocities refer to the fast P1-wave of the investigated samples, which arise from the coupling of the fluid and solid phase due to inertial effects. The experimentally determined phase velocities are investigated in a frequency range from 0.1 to $5.0 \mathrm{MHz}$, and determined from the TOFs of the received signals in the time domain according 


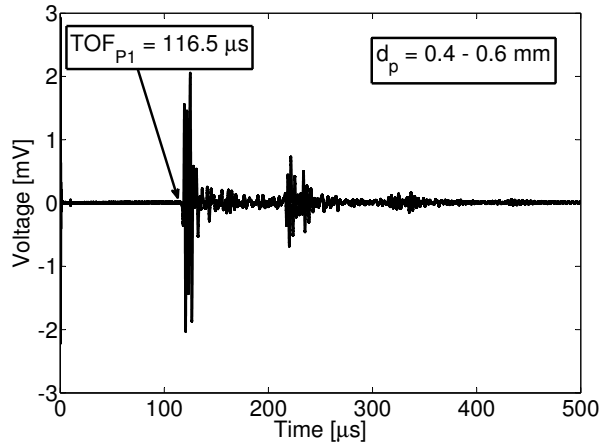

A.)

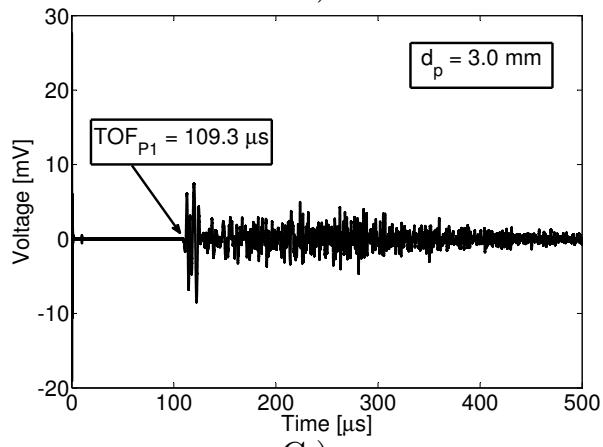

C.)

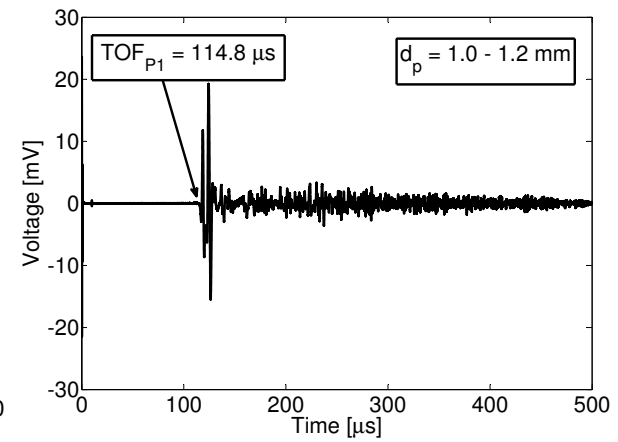

B.)

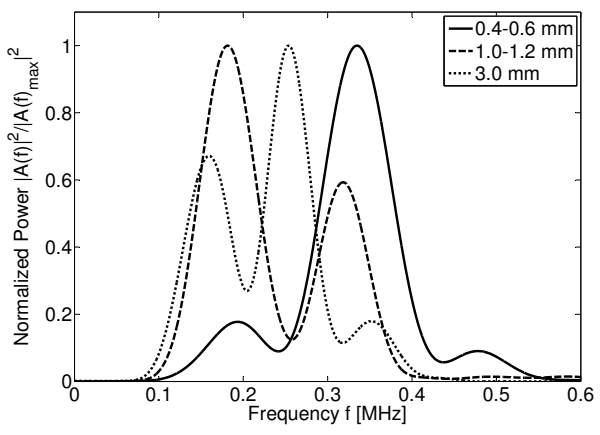

D.)

Figure 4.20: Received time signals through water-saturated samples with different glass bead diameters (using the experimental arrangement depicted in Figure 4.9 A.)): A.) $d_{p}=0.4-0.6 \mathrm{~mm} \mathrm{B.)} d_{p}=1.0-1.2 \mathrm{~mm} \mathrm{C}$.) $d_{p}=3.0 \mathrm{~mm}$. Figure D.) shows the peak-normalized Power Spectra of the coherent elastic parts. In each measurement, the acoustic transmitter is briefly stimulated with a square wave pulse with duration of $10 \mu \mathrm{s}$. 


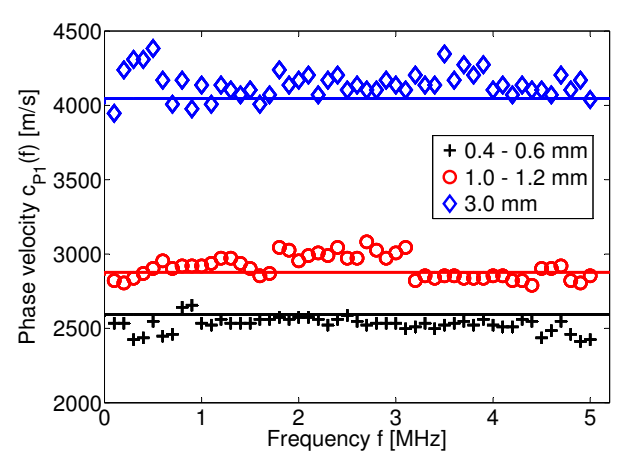

A.)

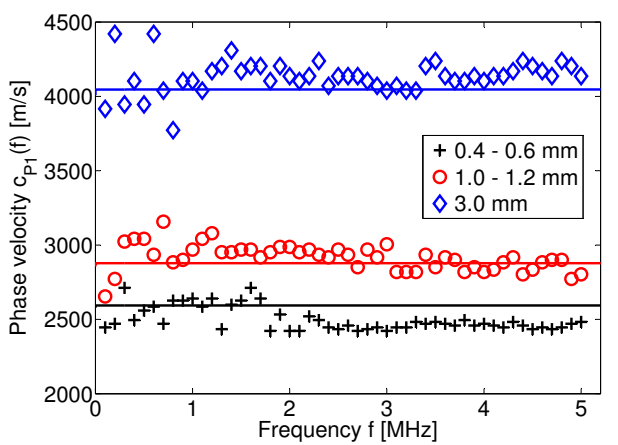

B.)

Figure 4.21: Comparison of experimentally and theoretically determined phase velocities for the fast p-wave of all investigated samples as function of frequency. The acoustic transmitter is excited with sinus-burst-signals: A.) of 1 cycle B.) of 10 cycles.

to Equation (4.38). For the calculations of the bulk velocities of the investigated samples, the TOF in water is determined at $132.5 \mu \mathrm{s}$. The theoretical curves from the Biot theory, represented by solid lines, describe well the experimentally determined velocities. All velocities remain almost constant in the plotted frequency range, which indicates that no dispersion effects are observed in the investigated frequency range.

Uncertainties of the measured velocities arise especially in the determination of the TOF, when the signal-to-noise ratio decreases and the deviation of the baseline is ambiguous. To overcome this problem, the phase velocities of the detected waves can be alternatively determined from the spectral ratio according to Equations (4.38) and (4.40). Figure 4.22 shows the phase velocities $c_{P 1}$, the specific attenuation $1 / Q_{P 1}$ and the length-normalized attenuation $\hat{\alpha}$ in a frequency range from 0.1 to $0.7 \mathrm{MHz}$, which corresponds approximately to the frequency spectrum of the analyzed coherent P1-wave. The frequency-dependent parameters are determined from the spectral ratio of the measurements in water only and with the sample. For the sake of clarity, only every 50th point of the spectrum is illustrated.

The phase velocities fluctuate around the reference velocity determined from water measurement and are certainly lower than the velocities determined in the time domain, cf. Figure 4.21. For all investigated samples, the specific attenuations $1 / Q_{P 1}$, depicted in Figure 4.22 (B.), first shows a decreasing trend, 


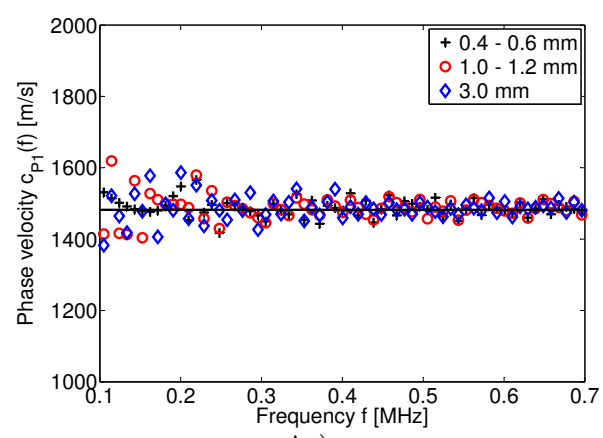

A.)

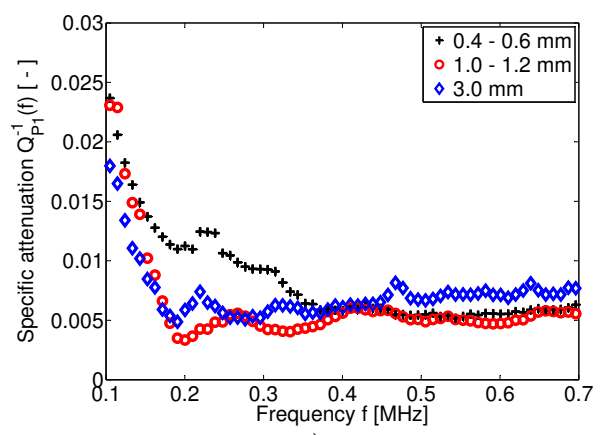

B.)

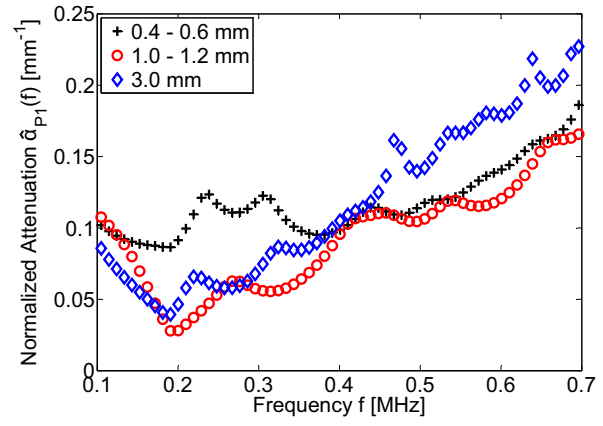

C.)

Figure 4.22: Phase velocities $c_{P 1}$, normalized attenuation $\hat{\alpha}$ and specific attenuation $1 / Q_{P 1}$ of the fast P1-wave of the investigated samples, whereby the piezoelectric transmitter is excited with a 1-cycle-sinus-burst-signal at $1 \mathrm{MHz}$. 


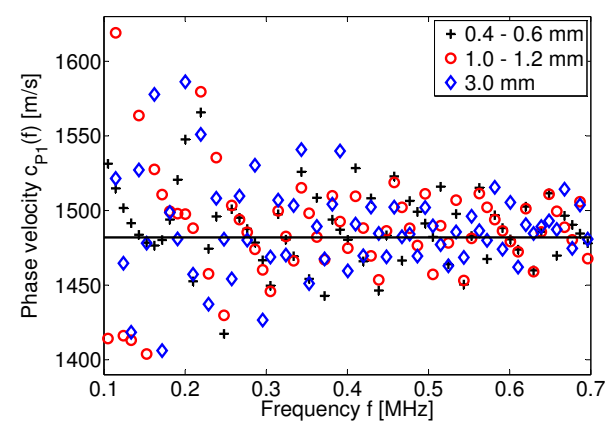

A.)

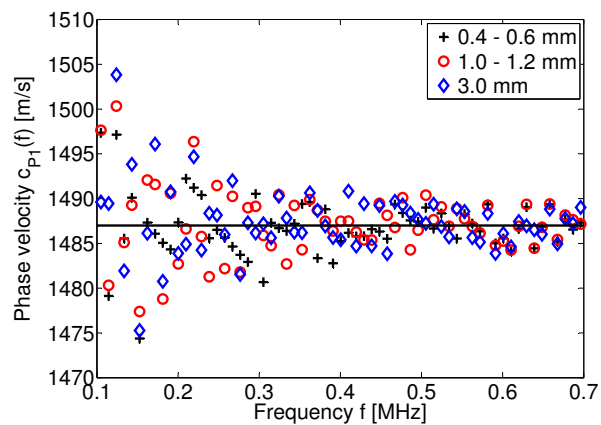

B.)

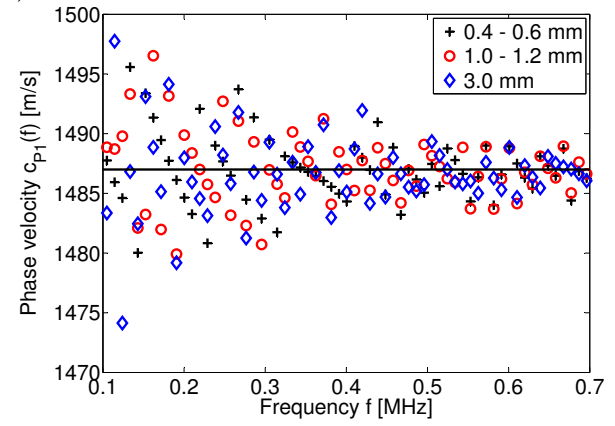

C.)

Figure 4.23: Phase velocities of the investigated samples, whereby the piezoelectric transmitter is excited differently with major frequency of $1 \mathrm{MHz}$ : A.) 1-cycle-sinus-burst B.) 10-cycle-sinus-burst C.) 1-cycle-Ricker-wavelet. Different symbols correspond to different diameters, while the solid lines give the reference velocity in water.

and finally remain almost constant up to $0.7 \mathrm{MHz}$. The normalized attenuations $\hat{\alpha}$ of the fast P1-wave, illustrated in Figure 4.22 (C.), decrease from 0.1 to $0.2 \mathrm{MHz}$, and then continuously increase from approximately 0.4 to $0.7 \mathrm{MHz}$. The frequency-dependent attenuation of the sintered sample with $d_{p}=3.0 \mathrm{~mm}$ is greater in the range from 0.4 to $0.7 \mathrm{MHz}$, compared to the other samples. This indicates the higher attenuation properties of the longer sintered sample in this frequency range. However, the sample with the smallest beads shows higher attenuation properties for lower frequencies $(f<0.4 \mathrm{MHz})$, cf. Figure 4.22 (B.-C.). 


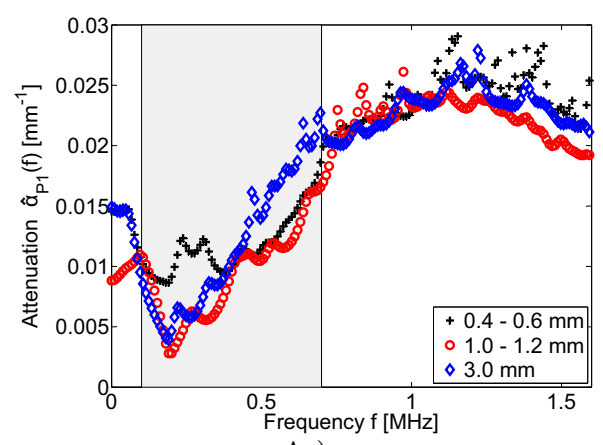

A.)

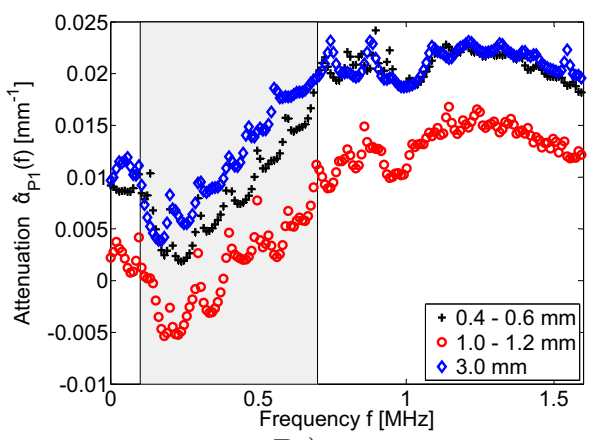

B.)

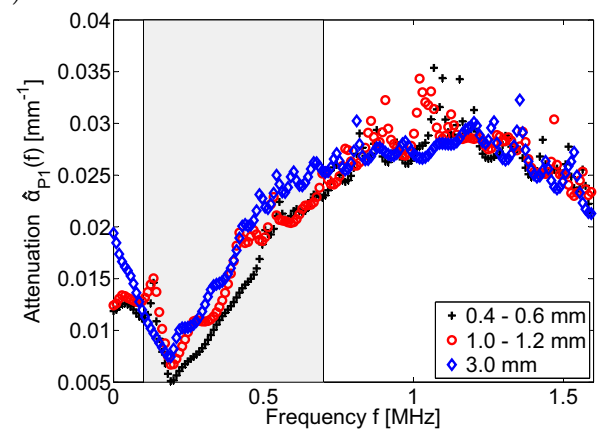

C.)

Figure 4.24: Normalized frequency-dependent attenuation $\alpha_{P 1}$ of the coherent fast P1-wave of the investigated samples at different excitation signals: A.) 1cycle-sinus-burst signal B.) 10-cycle-sinus-burst signal C.) 1-cycle-Ricker wavelet.

In order to demonstrate, that the frequency-dependent parameters highly depend on the input signal, used to excite the acoustic piezo-electric transmitter, Figures 4.23 and 4.24 show the phase velocities and attenuation at different input signals. Figure 4.23 shows, that the fluctuations of the phase velocities $c_{P 1}$ differ, when different input signals are used. Similar observations can be made for the normalized attenuations $\hat{\alpha}$, cf. Figure 4.24. The gray shaded area shows the approximate frequency range of the analyzed coherent wave. The normalized attenuations in Figure 4.24 (A.-C.) show a clearly increasing trend with an increasing frequency. The slope of the attenuations provide information about the signal quality. The signal quality, often referred as quality factor $Q_{P 1}$, increases with increasing slope of the normalized attenuations $\hat{\alpha}$. 


\subsection{Conclusion}

In summary, we have demonstrated that the Biot-theory can be successfully applied to water-saturated sintered samples with different macro- and microscopic features. For the proper prediction of the dispersion properties and TOFs of the investigated samples, several system parameters are required for which $\mathrm{X}$ ray Computer Tomographic images are used. For all investigated samples, the fast P1-wave could be detected and verified with Biot's theory, while the slow P2-wave is highly damped or superimposed with (multiple-) scattered waves ${ }^{3}$ as will be discussed in chapter 5 . We have demonstrated that the (low-frequency) coherent part of the detected wave is well described by the fast P1-wave in Biot's theory, while the (high-frequency) incoherent part has a diffusive character. A good agreement between experimentally and analytically determined TOFs and phase velocities of the fast P1-waves could be achieved.

It has been found out that high-frequency component of the ultrasonic broadband signal is filtered out or converted to (multiple-) scattered waves by the porous sintered glass bead samples. With regard to the coherent wave part, the porous sintered samples act as lowpass filter, which allow the low-frequency component of the broadband signal to pass through the sample and filter out the high-frequency component. Longer sintered samples with larger glass particles show stronger filter properties with lower cut-off frequencies than weakly sintered samples with smaller particles. A decrease of the cut-off frequency with increasing glass bead diameter and sinter duration could be proved. Compared to the reference measurement in water, a clear frequency- and time-shift of the peaks to lower frequencies in the power spectra and spectrograms could be detected for the investigated samples.

The dispersion parameters, phase velocity $c_{P h}$, specific attenuation $1 / Q$ and normalized attenuation $\hat{\alpha}$ have been investigated in a broader frequency range for different excitation signals. They are not only dependent on the investigated samples, but also on the excitation signals used to stimulate the piezo-electric acoustic transmitter, for instance in the relevant frequency range of the coherent pulse a clear increase of the normalized attenuation with increasing frequency was determined. The increase of the frequency-dependent attenuation is dependent on the electrical input signal used to excite the transmitter.

Furthermore, the received signals strongly depend on the frequency of the generated wave, especially with regard to the stronger presence of (multiple-) scattered waves. The amplitude of the coherent wave decreases with increasing frequency of the generated wave, while the amplitudes of the (multiple-) scattered waves are increasing. The proportion, which is converted to (multiple-) scattered waves, increases with increasing frequency of the generated wave.

\footnotetext{
${ }^{3}$ especially for the sintered samples with $d_{p}=1.0-1.2 \mathrm{~mm}$ and $d_{p}=3.0 \mathrm{~mm}$
} 


\subsection{Acknowledgement}

We acknowledge support from the research program of the Stichting voor Fundamenteel Onderzoek der Materie (FOM), which is financially supported by the "Nederlandse Organisatie voor Wetenschappelijk Onderzoek" (NWO), (project number: 09iPOG14-2).

\subsection{Appendix}




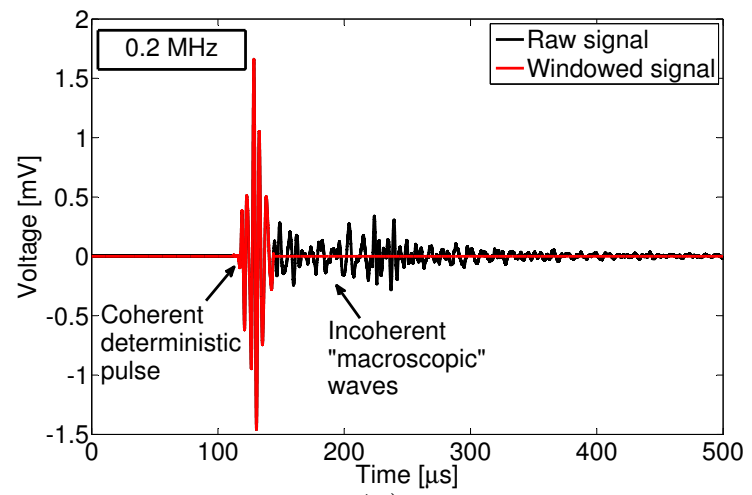

A.)

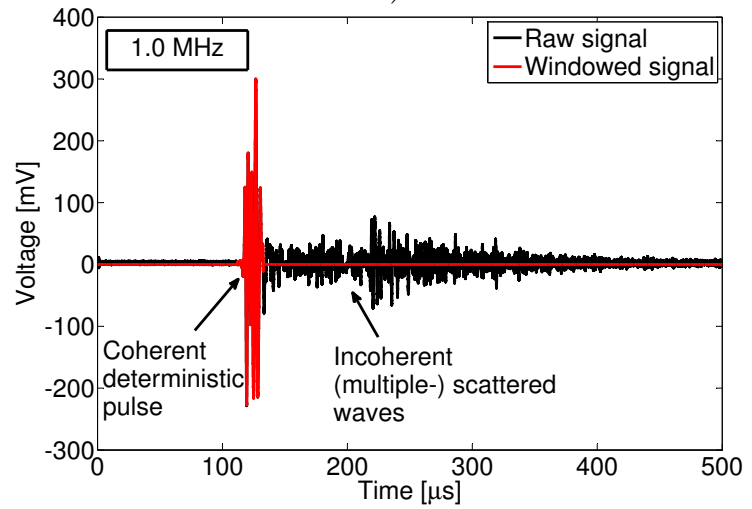

B.)

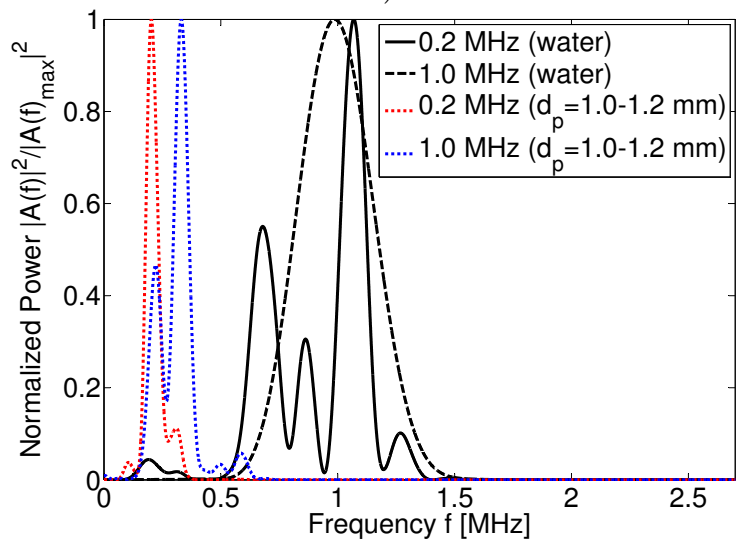

C.)

Figure 4.25: Received time signals of the generated low- (A.) and high-frequency (B.) wave with corresponding power spectra of the windowed signal. The piezoelectric transmitter is excited with a 1-cycle-sinus burst signal. The power spectra of the coherent parts of received signals with the corresponding reference measurement in water are shown in C.). A so-called Tukey-Win is used to separate the coherent part of the signal from the remaining part. 


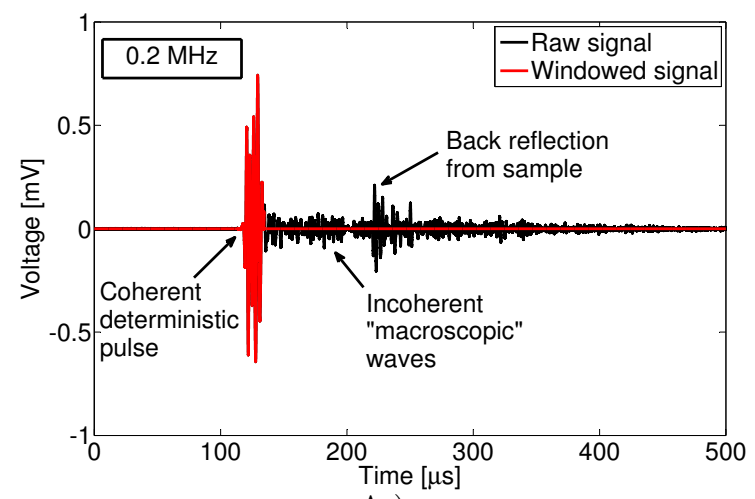

A.)

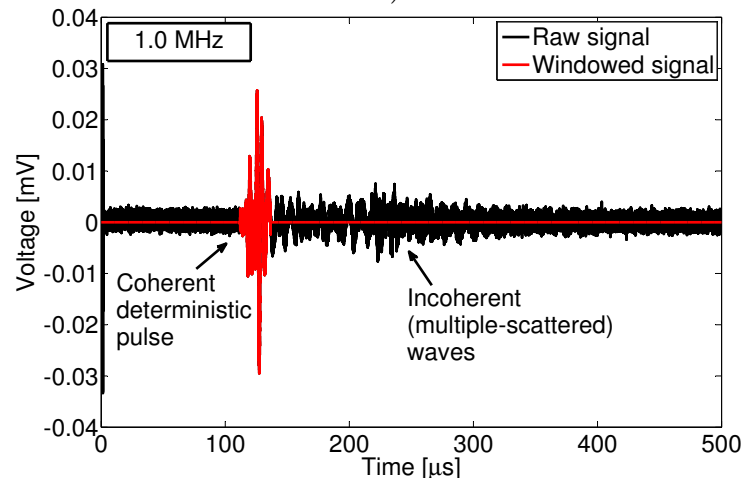

B.)

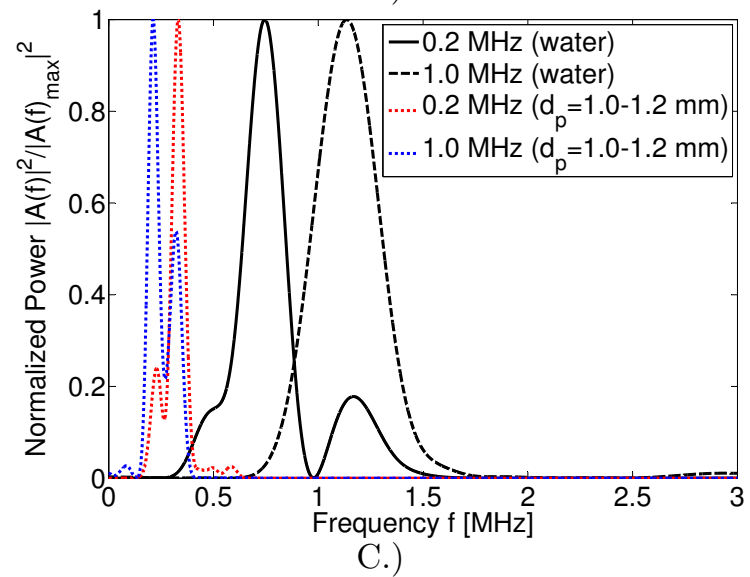

Figure 4.26: Received time signals of the generated low- (A.) and high-frequency (B.) wave. The piezo-electric transmitter is excited with a 1-cycle-Ricker-wavelet. The power spectra of the coherent parts of received signals with the corresponding reference measurement in water are shown in C.). A so-called Tukey-Win is used to separate the coherent part of the signal from the remainig part. 


\section{CHAPTER 5}

\section{Wave Propagation in \\ Sintered Porous Glass Bead Systems: High-Frequency \\ range}

In this study, incoherent transport of ultrasound waves in a water-saturated sintered glass bead packing is investigated experimentally. The spectral energy density associated with speckle-like (multiple-) scattered high-frequency waves is explained by diffusive processes. In accordance with classical predictions a diffusion coefficient of $D=0.38 \mathrm{~m}^{2} / \mathrm{s}$ and a quality factor of $Q=105$ could be determined from the diffusion approximation of the time-dependent intensity curve to the ensemble averaged data obtained from the ultrasound experiments. In this way, we demonstrate that the energy dissipation of (multiple-) scattered waves in watersaturated sintered glass bead systems are quantitatively and qualitatively well described by a diffusion model.

\subsection{Introduction}

Fluid-saturated sintered glass bead packings are strongly heterogeneous and multiscale and -phase systems. The heterogeneities due the random distribution of the glass beads in the solid matrix can lead to strong scattering of ultrasound waves at wavelengths in the order of the glass beads $\left(\lambda \approx d_{p}\right)$. The scattering of 
ultrasonic waves is further enhanced through micro cracks resulting from rapid cooling processes during sintering. Therefore, the use of relatively high-frequency ultrasound waves (in the $\mathrm{MHz}$ range) can provide useful information about the microstructure of strongly disordered inhomogeneous materials.

Previous investigations showed that the diffusion approximation can be successfully applied to the wave propagation under the conditions of strong multiple scattering in randomly distributed dry and wet glass bead packings [92]. Griffiths et al. [68] used diffusive ultrasound to investigate non-consolidated granular materials in different saturation gases and determined the frequency-dependent diffusion parameters. However, in their investigations the scattering medium was directly excited using contact transducers.

In this study, the diffusive ultrasound experiments are extended towards fluidsaturated sintered glass bead systems, whereby water is used as coupling medium and pore fluid. The applicability of the diffusion model, in which the wave interference is completely ignored, is tested on the incoherent wave part of the received ultrasound signal. Using the diffusion approach we determine the diffusion coefficient and quality factor in one disordered fluid-saturated sample consisting of sintered glass beads and water.

In section 6.3 the experimental procedure and set-up for the performed ultrasound experiments is briefly described. In section 5.3 the diffusive wave transport of ultrasound is introduced and approximated to the results obtained from the measurement of a water-saturated sample with glass bead sizes around $1.1 \mathrm{~mm}$. The observations and results are finally concluded in section 5.4.

\section{$5.2 \quad$ Experiments}

The experimental set up is a classical configuration to carry out immersion ultrasound measurements according to the transmission technique. Two immersion broadband transducers with central frequencies of $1 \mathrm{MHz}$ (Olympus, Panametrics NDT, V303-SU) are placed at the front and back of the porous sintered sample. The immersion transducers are not in direct contact with the investigated sample. The distance between the transducers and the porous sample is fixed at approximately $73 \pm 0.4 \mathrm{~mm}$. The ultrasound experiments are performed in a specially developed measuring cell made of acrylic glass. Prior to the ultrasound experiments, the measuring cell, including the porous sample, is rinsed out with carbon dioxide and filled with de-aired and filtered water, in order to achieve an optimal saturation of the sample. A detailed description of the measuring cell is described in [69]. A 10-cycle sinus burst excitation at a frequency of $0.9 \mathrm{MHz}$ is applied to the P-wave source transducer (Tektronix model AFG 3101). The narrow band excitation corresponds to the product of the acoustic wave number and bead diameter $k d_{p}=\omega d_{p} / c_{P 1} \approx 2.2$ with $c_{P 1}=2872.5 \mathrm{~m} / \mathrm{s}$ as the bulk velocity 
and $d_{p}=1.1 \mathrm{~mm}$ as the glass particle diameter forming the solid frame. In these high frequency ranges, a strong ultrasound scattering of the investigated medium is expected, where the wavelength of the ultrasound is only about $\lambda=3.2 \mathrm{~mm}$ and thus in the order of the particle diameter $d_{p}$. The received time signal is displayed and digitized on a $300 \mathrm{MHz}$-storage oscilloscope (ZTec 4441, maximum sample rate $800 \mathrm{MS} / \mathrm{s}$, vertical resolution $14 \mathrm{bit}$ ).

Figure 5.1 (A.) shows the typical received time signal of a water-saturated sintered glass bed sample with bead diameters between 1.0 and $1.2 \mathrm{~mm}$. The investigated cylindrical sample (with diameter and length $50 \mathrm{~mm}$ ) has an open pore structure and a porosity of $31.87 \%$. The time signal is basically composed of a coherent pulse arriving at the leading edge and an incoherent part mainly composed of speckle-like multiple-scattered waves, which are highly sensitive to the underlying microstructure of the investigated sample. For the diffusion approximation, only the incoherent wave part is considered. The dominant energy component of the transmitted wave is at a high frequency, which has a multiply scattered behavior approaching a diffusive regime $[92,162,163]$. In the diffusive regime the shear waves dominate the scattered wave field $[80,92]$. Using temporal windows for the calculations of the power spectra of the coherent and incoherent parts reveal that the frequency content of the coherent wave part is considerably lower than that of the incoherent part, cf. Figure 5.1 (C.). However, from the comparison between the power spectra of the total received signal and the incoherent part, a clear visible difference between both spectra appears, cf. Figure 5.1 (C.). The spectrum of the coherent pulse does not coincide with the low-frequency part of the total power spectrum. The incoherent part contains also a low-frequency wave part, which for instance results from the back reflection of the sample or from the occurrence of the so-called slow P2-wave [24, 25]. These waves commonly have the same frequency content as the coherent pulse [91]. Therefore, for an optimal separation of the high-frequency incoherent part from the remaining part of the received time signal, a highpass filter is applied to the raw signal. Figure 5.1 (B.) shows the highpass-filtered time signal, whereby the cut-off frequency has been chosen at $0.7 \mathrm{MHz}$ at which the low-frequency coherent pulse at the leading edge disappears. The corresponding power spectra of the highpass-filtered signal is shown in Figure 5.1 (D.). It can be clearly seen that the low-frequency part $(f<0.65 \mathrm{MHz})$ is filtered out.

\subsection{Diffusive wave propagation}

From the time signal, depicted in Figure 5.1 (A.), we can see that most of the energy transport through the water-saturated sintered glass bead sample is caused by scattered waves occurring in the incoherent wave part of the received time 


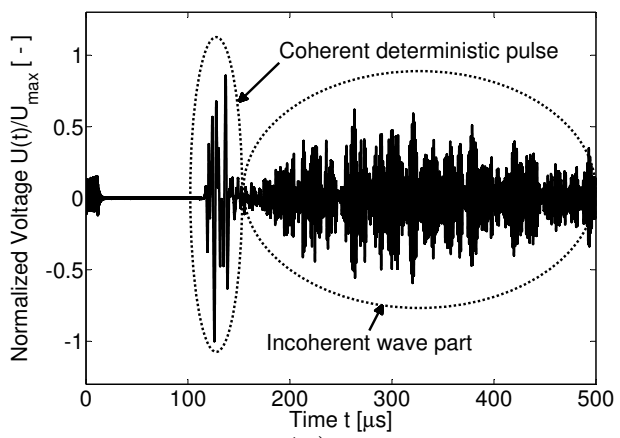

A.)

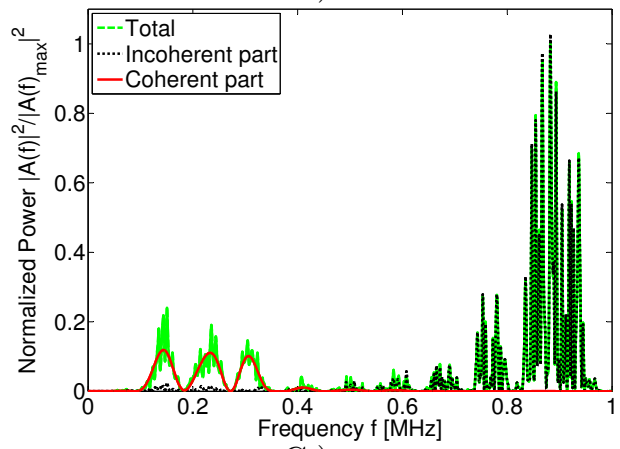

C.)

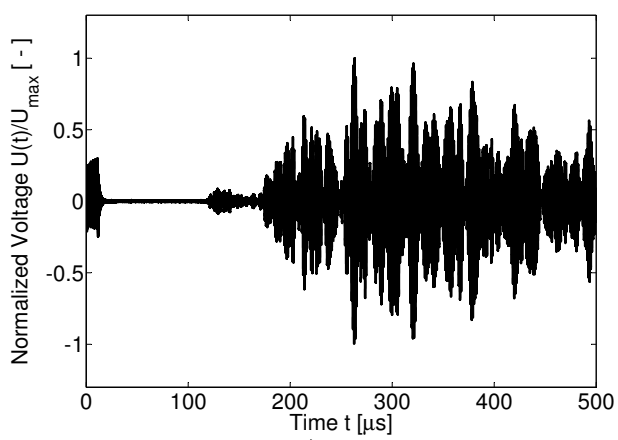

B.)

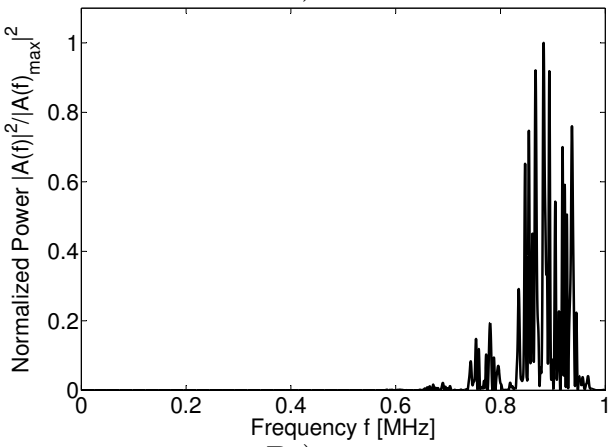

D.)

Figure 5.1: Received time signal in raw state (A.) and after highpass filtering (B.). The cut-off frequency is chosen at $0.7 \mathrm{MHz}$. The corresponding power spectra of the raw signal (C.) and of the highpass-filtered high-frequency incoherent part are shown below.

signals. Although it is challenging to describe the multiple scattering of classical waves, significant progress has been achieved through the use of the diffusive wave approximation $[46,135,138,186,188]$. In the diffusive modelling of multiplescattered waves the averaged wave intensity is treated as random walk process. The phase information is not considered and the wave propagation is described by a single diffusion coefficient as

$$
D=\frac{1}{3} c_{e} l^{*}
$$

where $l^{*}$ represents the average transport free path, which describes the mean distance the ultrasound wave travels before its propagation direction is random- 
ized by scattering "event" [92]. $c_{e}$ is the averaged ballistic velocity, at which the energy of the scattered waves is transported through the porous sample-water system.

The diffusion method is based on the assumption that the spectral energy density of the scattered ultrasonic wave field is well described by a diffusion equation. The diffusive wave field is temporally and spatially incoherent with the incident wave and the field variables are assumed to be random [48]. According to [48, 187] the diffusion equation that describes the time evolution of the spectral energy density $\langle U(z, t, f)\rangle$ of an ultrasonic wave field can be formulated as

$$
\frac{\partial\langle U(z, t, f)\rangle}{\partial t}=D \Delta\langle U(z, t, f)\rangle-\sigma\langle U(z, t, f)\rangle+P(z, t, f) \quad \bigvee \mathrm{z} \in \mathbb{B}
$$

where $P(z, t, f)$ is the spectral energy density source term (forcing condition) and $\sigma(f)$ the dissipation rate. $\mathbb{B}$ describes the elastic isotropic body of the scattering medium. The frequency-dependent diffusion coefficient $D(f)$ is very sensitive to the microstructure, but is assumed to be independent from the direction. Fast spatial variations in amplitude and phase, which are typical for a diffusive wave field, converge to zero when they are ensemble averaged over various configurations $[48,137,180]$. This property of waves is known as phase cancellation. It should be noticed that the energy in the coherent wave part is transferred to the incoherent wave part consisting of (multiple-) scattered waves with increasing frequency. No energy is lost in scattering and scattering has no contribution to the dissipation rate $\sigma$ [48]. A higher diffusion coefficient $D$ results in faster diffusion of energy transported in the multiple scattered waves, whereas an increasing scattering probability is associated with slowing down of the diffusive process and decreasing the diffusion coefficient. As highlighted by [136], the energy density measured on the same axis as the source at the distance $z=l$ can be approximated by a $1 \mathrm{D}$ solution to plane waves at various frequencies in the scattering sample. Under the assumption that the cell walls are perfectly reflecting at $z=0$ and $z=l$, Jia et al. [92] has proposed a simple solution for the time-dependent intensity profile based on a Fourier series approach as presented in [42]

$$
I(t)=-\left.D \frac{\partial U}{\partial z}\right|_{z=0} ^{z=l}=\frac{c_{e} U_{0}}{2 l} e^{-t / \tau_{a}} \sum_{n=0}^{\infty} \frac{(-1)^{n}}{\delta_{n}} \cos \left(n \pi l^{*} / l\right) e^{-D(n \pi)^{2} t / l^{2}},
$$

where $U_{0}$ is the energy of the transmitted scattered waves and $l$ the sample length. $\delta_{n}=2$ for $n=0$, otherwise $\delta_{n}=1$. $\tau_{a}$ describes the inelastic absorption time and can be expressed in terms of the quality factor of the scattered waves as

$$
\tau_{a}=\frac{Q}{2 \pi f} .
$$


Ultrasound experiments are commonly used to measure the diffusion coefficient and the quality factor from the time evolution of the transmitted high-frequency scattered waves. In this respect, the measured transmitted acoustic intensity is determined by ensemble averaging the square of the envelope of the scattered sound field, which is represented in the incoherent wave part of the received time signal [68, 93, 138, 180], see Figure 5.1 (A.-B.). Figure 5.2 shows a typical result of the evaluation of scattered ultrasound waves in a water-saturated glass bead sample. The measured normalized intensity curve results from the averaging of

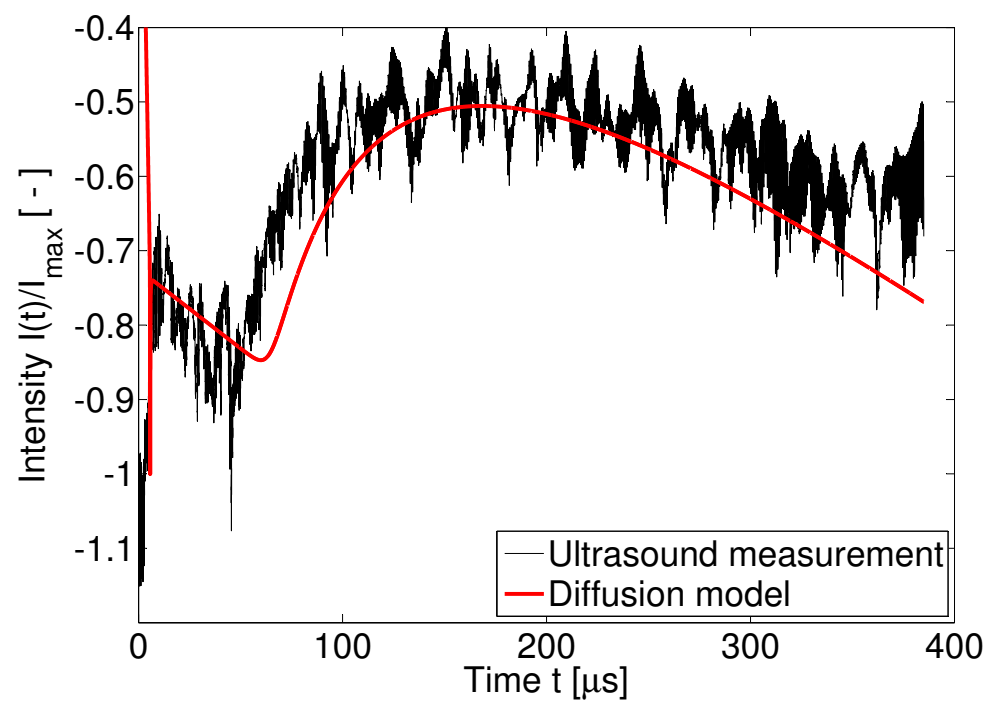

Figure 5.2: Comparison of normalized Intensities obtained from ultrasound measurements and diffusion model. The measured averaged intensity curve is determined from 10 independent measurements. The piezo-electric acoustic transmitter is excited with a 10-cycle-sinus burst at $0.9 \mathrm{MHz}$. The diffusion coefficient and quality factor is determined by fitting the diffusion model the measured averaged intensity profile at $D=0.38 \mathrm{~m}^{2} / \mathrm{s}$ and $Q=105$.

ten independent ultrasound experiments and is compared with the theoretical intensity curve, represented by the red solid line and determined from the solution of the diffusion equation given in (5.3). The ultrasound measurements are obtained from five different samples with the same glass bead diameters and sintering treatment. To ensure a well defined ultrasonic frequency, a sinus-burst-signal with 10 cycles is used. The frequency of the generated quasi-monochromatic wave has been chosen at $0.9 \mathrm{MHz}$. The diffusion approximation, which is normalized 
Table 5.1: Input parameters for the diffusion model and results obtained from the fit of the theoretical time-dependent intensity curve to measurement data.

\begin{tabular}{lcc}
\hline Parameter & Unit \\
\hline \hline Sample length $l$ & {$[\mathrm{~mm}]$} & 50 \\
Energy transport velocity ${ }^{[A]} c_{e}$ & {$[\mathrm{~m} / \mathrm{s}]$} & 956 \\
Energy of transmitted scattered & {$\left[\mathrm{cV}^{2}\right]$} & 113.6 \\
waves $^{[B]} U_{0}$ & {$[\mathrm{MHz}]$} & 0.9 \\
Frequency $f$ & {$[-]$} & 50 \\
Number of speckles $N$ & {$\left[\mathrm{~m}^{2} / \mathrm{s}\right]$} & 0.38 \\
\hline Diffusion coefficient ${ }^{[C]} D$ & {$[-]$} & 105 \\
Quality factor ${ }^{[C]} Q$ & {$[\mathrm{~mm}]$} & 1.2 \\
Mean transport free path ${ }^{[C]} l^{*}$ & & \\
\hline \hline
\end{tabular}

$[A]$ : Experimentally determined energy transport velocity obtained from the average Time Of Flight (TOF) of the received time signals of ten independent ultrasound measurements transmitted through the water-sample-water system. The ultrasound measurements are obtained from five different samples, whereby the transmitter and receiver have been changed for each investigated sample.

$[B]$ : Determined from the integration of the measured averaged intensity profile according to the trapezoidal rule with step size $(b-a) / n$, where $a$ and $b$ describe the integration limits and $n$ the number trapezoidal areas.

${ }^{[C]}$ : Determined from the fit to the theoretical time-dependent intensity profile to experimental data.

to the peak of the intensity, is calculated by using the diffusion coefficient and quality factor as fit parameter. For the water-saturated sintered sample with bead diameters between 1.0 and $1.2 \mathrm{~mm}$ the best fit is obtained with a set of parameters $D=0.38 \mathrm{~m}^{2} / \mathrm{s}$ and $Q=105$. Note, that for the fit of Equation (5.3) a new temporal axis is defined with $t=T O F=0$ as the beginning of the penetration of the ultrasound wave into the porous sample. Further input parameters used to predict the time-dependent intensity curve and important results are summarized in Table 5.1. The mean transport free path of the ultrasound wave is determined according to Equation (5.4) at $1.2 \mathrm{~mm}$. This means that the ultrasound wave changes its direction and is being randomized every approximately $1.2 \mathrm{~mm}$ in the porous sample, which satisfactory close to the particle diameter. 


\subsection{Conclusion}

In summary, we can conclude that the diffusive wave propagation model can be successfully applied to the incoherent wave part of the received time signals from ultrasound transmission measurements in water-saturated sintered glass packings. A good agreement between experimentally and theoretically time-dependent intensity curves of the scattered waves could be achieved. For the generated sinusoidal signal of 10 cycles with $0.9 \mathrm{MHz}$, the frequency-dependent diffusion coefficient could be determined at $D=0.38 \mathrm{~m}^{2} / \mathrm{s}$. The transport mean free path of the ultrasound wave was determined from the measured Diffusion coefficient as $l^{*}=1.2 \mathrm{~mm}$. This result corresponds approximately to glass bead sizes, which the porous sintered sample is composed of. The quality factor, which describes the time-dependent attenuation of the (multiple-) scattered wave part, could be determined as $Q=105$.

Future work should therefore include the evaluation of the diffusion coefficient and the quality factor of scattered waves for different frequencies and pore fluids to further understand the relevance of the diffusive wave model for the acoustical wave propagation in sintered glass bead systems.

\subsection{Acknowledgement}

We acknowledge support from the research program of the Stichting voor Fundamenteel Onderzoek der Materie (FOM), which is financially supported by the "Nederlandse Organisatie voor Wetenschappelijk Onderzoek" (NWO), (project number: 09iPOG14-2). 


\section{CHAPTER 6}

\section{A Contribution to Biot's Slow P2-Wave: An Experimental Study in Fluid-Saturated Porous Sintered Glass Bead Systems}

The detection of slow P2-waves in fluid-saturated porous media is in practice diffcult due to it's strong attenuation and superposition with different wave types (i.e. scattered waves). In the most cases the technical requirements necessary for the observation of the slow P2-wave (for instance the vertical resolution of the used oscilloscope) are not sufficient. In this study, the applicability of Biot's theory is investigated experimentally on a fluid-saturated porous sintered glass bead sample. The Biot theory is used to identify the slow P2-wave in ultrasonic measurements. Different Newtonian fluids (water and silicone oil) are used to saturate the same glass bead sample, in order to check the consistency of Biot's theory. While the slow P2-wave could be clearly identified for the water-saturated sintered sample, the detection of the slow P2-wave in silicone oil-saturated sample is hampered by the higher viscous coupling property with the solid frame and stronger attenuation coefficient as compared to water. A good agreement between theoretically and 
experimentally determined Time Of Flights (TOF) of the detected waves is reported. However, the frequency-dependent attenuation obtained from Biot theory is not sufficient to describe the attenuation in the studied systems, which gives the ultrasound experiments a great importance for a better understanding of the physical interactions between the fluid and solid matrix - in our case composed of sintered glass beads.

\subsection{Introduction}

The investigation of ultrasonic wave propagation in fluid-saturated porous media is often interpreted in terms of Biot's theory with varying degree of success $[24,25,27,107]$. The detection and evaluation of the slow P2-wave, which is highly sensitive on the underlying microstructure of the porous medium and it's saturating pore fluid, has always occupied the research in the field of acoustical wave propagation in porous media $[36,74,84,99,104,105,110,111,172]$. However, the application of Biot's theory on real experimental data proved to be very difficult, since, unlike the fast P1-wave, the detection of the slow $\mathrm{P} 2$-wave is in the most cases not directly visible due to it's strong attenuation and superposition (time-overlapping) with different wave types, like (multiply-) scattered waves or/and the fast P1-wave. Practical observation of single wave modes can become even more difficult, due to the required differentiation into time or frequency domain or too low sensitivity, cf. [101]. Therefore, experimental observations of the slow Biot-waves required a long time, cf. [144]. Two longitudinal wave modes can also be observed in bones $[114,172]$. Bones, however, have a strongly anisotropic structure, due to adaptation to mechanical loading. For this reason, the slow P2-wave only occurs in directions where fluid flow is possible along the bone channels [84, 105, 114].

In this study, the general problems addressing the detection of the slow P2-wave are outlined and discussed. The presence of the slow P2-wave is experimentally confirmed in fluid-saturated artificial porous media composed of sintered glass beads. In contrast to common rock samples and bones, the microscopic and macroscopic features, which favor the propagation of slow P2-wave, can be influenced by the selection of certain glass beads and special sintering treatments. The acoustical wave propagation in porous sintered glass bead sample is analyzed extensively for different saturating fluids. The effect of the saturating fluid is investigated in detail with the goal of evaluating it's contribution to the presence of the slow P2-wave and the velocity and attenuation dispersion mechanisms.

In section 6.2 the mathematical frame work for the propagation of the fast and 
slow P-waves is briefly introduced and the frequency-dependent TOFs of both waves determined from the dispersion relation for different saturating fluids. In section 6.3 the experimental set-up and procedure is described. The results from the ultrasound experiments are presented in section 6.4. The final section 6.5 addresses the conclusion gained from the ultrasound experiments in water- and silicone oil-saturated sintered sample and gives an outlook for future work.

\subsection{Theoretical background}

\subsubsection{Biot model}

One of the most important features of the Biot model $[24,25,27]$ is the presence of the so-called slow P2-wave, often referred to as Biot-wave, which arises from the out of phase movement of the solid matrix and the fluid phase. In contrast, the fast $\mathrm{P} 1$-wave arises when the fluid and solid phase move in phase due to viscous or inertial coupling. In accordance with $[104,172]$ the dispersion relations for the propagation of both $\mathrm{P}$-waves can be formulated as an eigenvalue problem

$$
\operatorname{det}\left(\mathbf{A}_{P}-\bar{k}_{P}^{2} \mathbf{B}_{P}\right)=0
$$

with the matrices

$$
\mathbf{A}_{P}=\left[\begin{array}{ll}
\rho_{11} \omega^{2}-i \omega F(\omega) b_{0} & \rho_{12} \omega^{2}+i \omega F(\omega) b_{0} \\
\rho_{12} \omega^{2}+i \omega F(\omega) b_{0} & \rho_{22} \omega^{2}-i \omega F(\omega) b_{0}
\end{array}\right] \quad \text { and } \quad \mathbf{B}_{P}=\left[\begin{array}{ll}
P & Q \\
Q & R
\end{array}\right]
$$

The matrix $\mathbf{A}_{P}$ describes the frequency-dependent momentum exchange between the solid matrix and the saturating fluid due to inertial $\left(\propto \omega^{2}\right)$ or viscous $(\propto i \omega)$ effects with angular frequency $(\omega)$ and wave number $\bar{k}_{P}$ [104]. The densities in matrix $\mathbf{A}_{P}$ can be expressed in terms of measurable quantities of the propagating medium $\rho_{11}:=\left(1-\phi_{0}\right) \rho^{s R}-\rho_{12}, \rho_{22}:=\phi_{0} \rho^{f R}-\rho_{12}$ and $\rho_{12}:=\left(1-\alpha_{\infty}\right) \phi_{0} \rho^{f R}$. The porosity and tortuosity of the porous medium are introduced as $\phi_{0}$ and $\alpha_{\infty}$ and the true bulk densities of the solid and the fluid phase as $\rho^{s R}$ and $\rho^{f R}$. The viscous damping is usually expressed in terms of the intrinsic permeability $k_{z}^{s}$ of the porous medium as $b_{0}=\eta^{f R} \phi_{0}^{2} / k_{z}^{s}$. The index $z$ refers to the intrisic permeability in flow and propagation direction $z$. The frequencydependent correction factor for the damping according to Johnson et al. [95] reads as $F(\omega)=\sqrt{1-0.5 i \omega / \omega_{\text {crit }}}$ with a critical frequency $\omega_{\text {crit }}$ which characterizes the transition from a viscous dominated flow regime to an inertial dominated flow regime. The critical frequency is defined as $\omega_{\text {crit }}=f_{\text {crit }} / 2 \pi:=b_{0} / \rho_{22}$. The frequency-dependent phase velocities and attenuation coefficients of the $\mathrm{P}$-waves 
can be calculated on basis of the eigenvalue problem as

$$
c_{P 1, P 2}(\omega)=\frac{\omega}{\operatorname{Re}\left[\bar{k}_{P 1, P 2}(\omega)\right]} \quad \text { and } \quad \alpha_{P 1, P 2}(\omega)=\operatorname{Im}\left[\bar{k}_{P 1, P 2}\right] .
$$

The generalized elastic parameters in matrix $\mathbf{B}_{P}$ can be determined from measurable quantities as

$R=\frac{K^{f}}{\phi^{E}} \phi_{0}^{2} ; Q=\frac{K^{f}}{\phi^{E}}\left(\frac{K}{K^{s}}+1-\phi_{0}\right) ; P=K+\frac{4}{3} G+\frac{K^{f}}{\phi^{E}}\left(\frac{K}{K^{s}}+1-\phi_{0}\right)$

As can be seen in Equation (6.4), the elastic parameters depend not only on the bulk modulus of the skeleton frame $K$, the fluid $K^{f}$ and the solid grains $K^{s}$, but also on the effective porosity $\phi^{E}:=\phi_{0}+K^{f} / K^{s}\left(1-\phi_{0}-\frac{K^{f}}{K^{s}}\right)$.

\subsubsection{Immersion ultrasonic measurements}

The frequency-dependent phase velocity in immersion ultrasonic measurements is usually determined as

$$
c_{P 1, P 2}(\omega)=c_{\mathrm{ref}}\left(1-\frac{c_{\mathrm{ref}}}{l} \Delta T O F(\omega)\right)^{-1},
$$

whereby the time difference is defined as the difference in arrival times between sample and reference measurement $\triangle T O F(\omega):=T O F(\omega)-T O F_{\text {ref. }} c_{\text {ref }}$ and $T O F_{\text {ref }}:=L / c_{\text {ref }}$ are the speed of sound and the arrival time obtained from the reference measurement, where a no dispersive sample is positioned between the transducers. $L$ and $l$ represent the distance between the transducers and the length of the porous sample, see Figure 6.1.

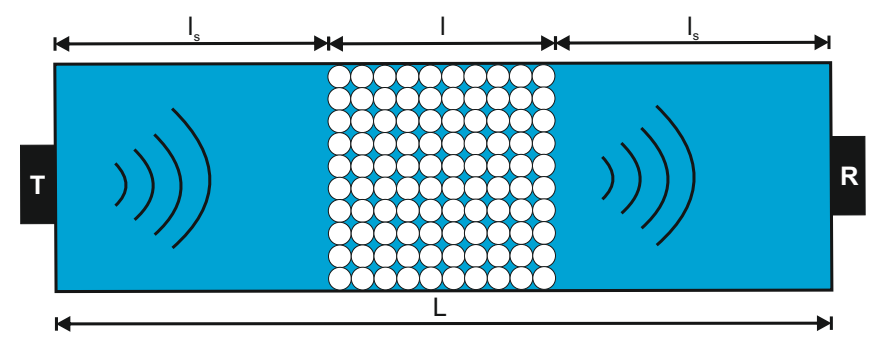

Figure 6.1: Simplified sketch of the measuring cell with length specifications used for ultrasound measurements. 


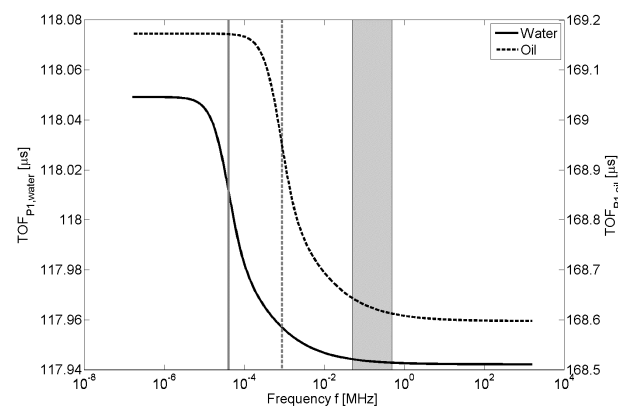

A.)

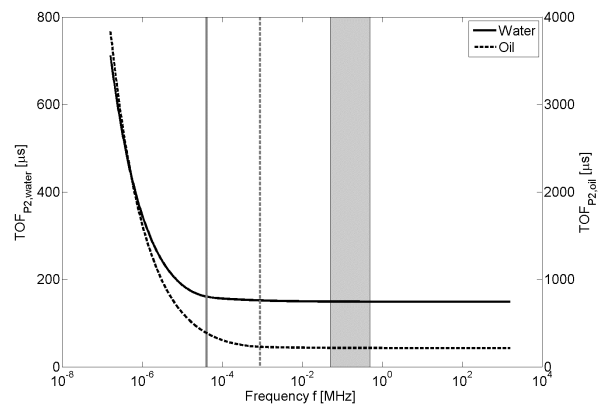

B.)

Figure 6.2: Frequency-dependent TOFs of the fast P1- (A.) and slow P2-waves (B.) for the investigated porous sintered sample in silicone oil and water. The frequency-dependent TOFs are determined from the dispersion relations according to Equation (6.6). The vertical lines represent the critical frequencies of investigated sample in water and silicone and the gray-shaded area indicates the approximate frequency-range if the received ultrasound signals.

Combining Equation (6.5) and (6.3) yields the frequency-dependent arrival times of the P-waves

$$
\operatorname{TOF}_{P 1, P 2}(\omega)=T O F_{\text {ref }}+\frac{l}{c_{\text {ref }}}-\frac{l\left|\operatorname{Re}\left[\bar{k}_{P 1, P 2}(\omega)\right]\right|}{\omega} .
$$

Equation (6.6) combines the theoretical determination of the arrival times with the given geometrical boundary conditions of the ultrasound experiments and can be used to predict the arrival times of the P-waves and to identify the slow and fast $\mathrm{P}$-waves in ultrasound experiments. In this respect, Figure 6.2 shows the frequency-dependent arrival times of the fast and slow P2-waves for a water-and silicone oil-saturated sintered glass bead sample with glass particles between 0.4 and $0.6 \mathrm{~mm}$. For the theoretical predictions the dispersion relation formulated in (6.1) and the material parameters summarized in Table 6.1 are used.

\subsubsection{Prediction of Time of Flights}

While the frequency-dependent TOF curves for the fast P1-wave, shown in Figure 6.2 (A.), remain almost constant and hardly show any dispersion, the predicted TOFs for the slow P2-waves goes to infinity $\left(T_{P 2} \rightarrow \infty\right)$ for $\omega \rightarrow 0$, which indicate that the slow $\mathrm{P} 2$-wave, independent from the saturation fluid, shows a diffusive character below the critical frequency and cannot propagate 
in the low-frequency regime, cf. Figure 6.2 (B.). The critical frequencies of the water- and oil-saturated sintered sample are given by the vertical lines. The critical frequency of the oil-saturated sample is approximately 20 times higher due to the higher viscosity of the used silicone oil (Fragoltherm-X-160-A), see Table 6.1. However, it can be seen that in the relevant frequency range of the ultrasound experiments, represented by the gray-shaded area, the TOFs of the fast and slow P-waves remain, independent from the used pore fluid, constant and no dispersion is expected. In the relevant frequency range of the received ultrasound signals both $\mathrm{P}$-waves can theoretically propagate.

However, it should be noticed that the Biot theory does not take the scattering effects into account, which is a main problem in detecting the slow $\mathrm{P} 2$-wave in ultrasonic measurements. Scattering effects usually takes place when the wavelengths of the ultrasonic waves are of same the order as the glass particle, which the solid matrix is composed. Scattered waves are created if the dimensions of the heterogeneities are comparable to or lower than the wavelength $\left(\lambda \approx d_{p}, \lambda<d_{p}\right)$ $[107,137,138,163]$. To overcome or minimize this problem glass particles with diameters between 0.4 and $0.6 \mathrm{~mm}$ are used. Figure 6.3 (A.) shows the wavelength of the P1- and P2-wave transmitted through the water- and oil-saturated sample. One can see, that the wavelengths are greater than the particle diameter, represented by the black area. Note, that scattering effects do not only depend on the sizes of the used glass particles, but also on the sintering necks that can be different dependent on the treatment. Longer sinter durations or microcracks, resulting from rapid cooling after sintering, can also cause stronger heterogeneity of the samples and pore shape and thus enhances the scattering effects.

\subsection{Experiments}

Ultrasonic measurements have been performed according to the transmission method, where one transducer serves as a transmitter (sender) and the other as a receiver, see Figure 6.1. The experimental arrangement for transmission of ultrasound is shown in Figure 6.3 (B.). Broadband immersion transducers with central frequencies of $1 \mathrm{MHz}$ (Olympus, Panametrics-NDT, type V303-SU, 0.5 inch in diameter, see Ref. [4]) have been mounted opposite to each other in a specially developed multi-purpose measuring cell, which has been flooded with distilled water or silicone oil at $23.7 \pm 0.2^{\circ} \mathrm{C}$. The piezo-electric transmitter is driven by an arbitrary function generator (Tektronix model AFG 3101 with a time resolution of $8 \mathrm{ps}$ and a vertical resolution of $1 \mathrm{mV}$ ), whereby different electrical input signals, such as a single sinusoidal burst signal or a Ricker wavelet with voltages of $\pm 711 \mathrm{mV}$ at different frequencies are used. The electrical input signals are power-amplified with $55 \mathrm{~dB}$ (RF Power Amplifier, model E\&I 1040L, $10 \mathrm{kHz}-5 \mathrm{MHz}, 400 \mathrm{~W})$. The received signals are pre-amplified and displayed on 
Table 6.1: Material parameters used for Biot theory.

\begin{tabular}{|c|c|c|}
\hline Parameter & Unit & Water \\
\hline $\begin{array}{l}\text { Fluid temperature }{ }^{[A]} T^{f R} \\
\text { Viscosity } \eta^{f R} \\
\text { Density } \rho^{f R} \\
\text { Time Of Flight }{ }^{[A]} T O F_{\text {ref }} \\
\text { Speed of Sound }{ }^{[A]} c_{\text {ref }} \\
\text { Bulk modulus } K^{f} \\
\text { Critical frequency } f_{c r i t} \\
\text { Viscous frequency } f_{v}\end{array}$ & $\begin{array}{c}{\left[{ }^{\circ} \mathrm{C}\right]} \\
{[\mu \mathrm{Pa} \mathrm{s}]} \\
{\left[\mathrm{kg} / \mathrm{m}^{3}\right]} \\
{[\mu \mathrm{s}]} \\
{[\mathrm{m} / \mathrm{s}]} \\
{[\mathrm{GPa}]} \\
{[\mathrm{Hz}]} \\
{[\mathrm{MHz}]}\end{array}$ & $\begin{array}{c}23.9 \\
922 \\
998 \\
132.3 \\
1481 \\
2.22 \\
40.8 \\
0.46\end{array}$ \\
\hline Parameter & Unit & Silicone oil \\
\hline $\begin{array}{l}\text { Product name } \\
\text { Fluid temperature }{ }^{[A]} T^{f R} \\
\text { Viscosity }^{[B]} \eta^{f R} \\
\text { Density }{ }^{[B]} \rho^{f R} \\
\text { Time Of Flight }{ }^{[A]} T O F_{\text {ref }} \\
\text { Speed of Sound }{ }^{[A]} c_{\text {ref }} \\
\text { Bulk modulus } K^{f} \\
\text { Critical frequency } f_{c r i t} \\
\text { Viscous frequency } f_{v}\end{array}$ & $\begin{array}{c}{[-]} \\
{\left[{ }^{\circ} \mathrm{C}\right]} \\
{[\mu \mathrm{Pas}]} \\
{\left[\mathrm{kg} / \mathrm{m}^{3}\right]} \\
{[\mu \mathrm{s}]} \\
{[\mathrm{m} / \mathrm{s}]} \\
{[\mathrm{GPa}]} \\
{[\mathrm{Hz}]} \\
{[\mathrm{MHz}]}\end{array}$ & $\begin{array}{c}\text { Fragoltherm X-160-A } \\
23.6 \\
19200 \\
960 \\
197.6 \\
992 \\
0.94 \\
882.7 \\
9.27\end{array}$ \\
\hline Parameter & Unit & Sintered glass bead sample \\
\hline $\begin{array}{l}\text { Glass particle diameter }{ }^{[B]} d^{s} \\
\text { Density of solid grains }{ }^{[B]} \rho^{s} \\
\text { E-modulus of solid grains }{ }^{[B]} E^{s} \\
\text { Bulk modulus of solid grains } K^{s} \\
\text { E-modulus of solid frame } E \\
\text { Bulk modulus of solid frame } K \\
\text { Tortuosity factor }{ }^{[C]} \\
\text { Mean pore diameter }{ }^{[C]} \\
\text { Initial porosity }{ }^{[A]} \phi_{0} \\
\text { Effect. permeability }{ }^{[A]} k_{z}^{s}\end{array}$ & $\begin{array}{c}{[\mathrm{mm}]} \\
{\left[\mathrm{kg} / \mathrm{m}^{3}\right]} \\
{[\mathrm{GPa}]} \\
{[\mathrm{GPa}]} \\
{[\mathrm{GPa}]} \\
{[\mathrm{GPa}]} \\
{[-]} \\
{[\mu \mathrm{m}]} \\
{[\%]} \\
{\left[\mathrm{m}^{2}\right]}\end{array}$ & $\begin{array}{c}0.4-0.6 \\
2500 \\
59 \\
36.42 \\
6.62 \\
3.34 \\
1.46 \\
165.74 \\
37.04 \\
9.16 \times 10^{-10}\end{array}$ \\
\hline
\end{tabular}

$[A]$ : Measured values, see Refs. [69, 71]

$[B]$ : Manufacturer information, see Refs. $[1,6]$

${ }^{[C]}$ : Obtained from $1024 \times 1024$ x 2048 cuboid extracted from $\mu$ XRCT data, see Ref. [69] 


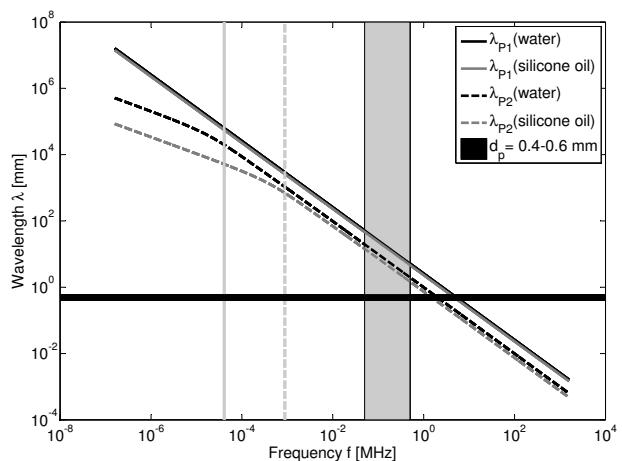

A.)

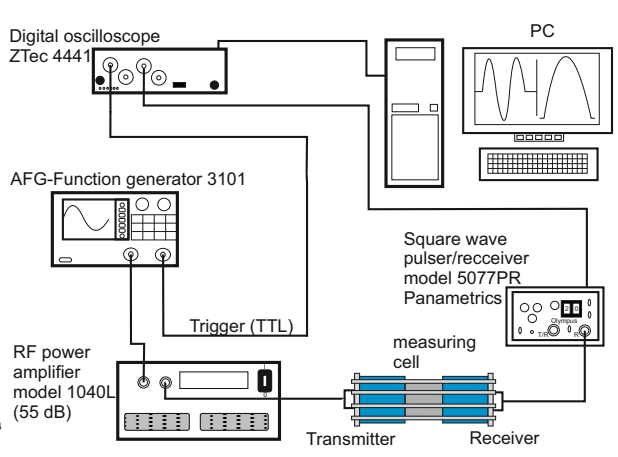

B.)

Figure 6.3: Frequency-dependent wavelength of the fast and slow P-wave in water- and silicone oil-saturated sintered glass bead specimen (A.). The diameter range of the glass particles is represented by the black area. The approximately frequency range of the received time signal is given by the gray-shaded area. The experimental set up for the ultrasound measurements (B.) according to the transmission method.

a storage oscilloscope (ZTec model 4441 with a vertical resolution of 14 bits). The cylindrical porous sample with a bulk diameter and length of $50 \mathrm{~mm}$ is positioned between the transducers. The distance between sample and transducers is fixed at $l_{s}=73 \mathrm{~mm}$ on both sides, so that $L=2 L_{s}+l=196 \mathrm{~mm}$, cf. Figure 6.1 . Note, that the angle of incidence of the ultrasonic wave, under the assumption of a plane wave, is perpendicular to the considered surface of the investigated sample, so that shear waves can be excluded.

\subsection{Results and discussion}

The raw time signals obtained from the ultrasound measurements of both waterand silicone oil-saturated sintered sample at different electrical input signals are shown in Figures 6.4 and 6.5 (A., B.). The time signals basically consist of a coherent impulse characterized by large amplitudes and an incoherent part arriving at later times, which consists of (multiply-) scattered waves, the slow $\mathrm{P} 2$-wave and reflected waves. Comparison of the arrival times obtained from Biot theory and ultrasound experiments confirms that the coherent pulse corresponds to the fast P1-wave, which mainly arises from the inertial coupling between solid and fluid phase, when both phases move in phase, see Table 6.2. 
Table 6.2: Comparison of theoretically and experimentally determined TOFs and SOSs of the slow and fast P-waves.

\begin{tabular}{l|c|c|c|c|c}
\hline \multirow{2}{*}{ Parameter } & Unit & \multicolumn{2}{|c|}{ Water } & \multicolumn{2}{c}{ Silicone oil } \\
\cline { 3 - 7 } & & Experiment & Theory & Experiment & Theory \\
\hline \hline Fast P1-wave $T O F_{P 1}$ & {$[\mu \mathrm{s}]$} & 117.4 & 117.9 & 169.6 & 168.6 \\
Slow P2-wave $T O F_{P 2}$ & {$[\mu \mathrm{s}]$} & 157.6 & 149.1 & - & 215.3 \\
\hline Fast P1-wave $c_{P 1}$ & {$[\mathrm{~m} / \mathrm{s}]$} & 2652.5 & 2577.0 & 2231.3 & 2334.0 \\
Slow P2-wave $c_{P 2}$ & {$[\mathrm{~m} / \mathrm{s}]$} & 846.7 & 990.2 & - & 737.0 \\
\hline
\end{tabular}

For the detection and a better visualization of the slow P2-wave, the raw signals are lowpass-filtered (with a cut-off frequency of $0.45 \mathrm{MHz}$ ). In this way the background noise and the (multiply-) scattered wave part is removed from the remaining part of the signal. While the slow P2-wave in the water-saturated sample is clearly visible after the lowpass-filtering, the slow P2-wave of the silicone oil-saturated sample cannot be clearly detected due to higher viscous coupling and attenuation by the pore fluid, cf. Figures 6.4 and 6.5 (C., D.). It should be stated, that in both cases the same porous sintered sample was used.

For comparison the reference signals in water and silicone oil are illustrated in Figures 6.4 and 6.5 (E., F.). The insets in Figures 6.4 and 6.5 (E., F.) show an enlargement of the transmitted useful signals determined from the reference measurements, where in addition to the TOFs and the amplitudes the signals shapes of the received signals can be seen. The received signal in silicone oil shows additional oscillations compared to water, although in both cases the acoustic transmitter was either excited with a 1-cycle-sinus-burst signal at $1 \mathrm{MHz}$ or a 1-cycle-Ricker-wavelet at 0.1 MHz. The Speed of Sound (SOS) in water and in silicone oil were determined at room temperature $\left(23.6-23.9^{\circ} \mathrm{C}\right)$ at $1481 \mathrm{~m} / \mathrm{s}$ and $998 \mathrm{~m} / \mathrm{s}$.

The comparison of the predicted and the measured arrival times of the slow P2wave in the water-saturated sample proves the detection of the Biot-wave, see Table 6.2. The time difference between experimentally and theoretically determined TOFs of the P2-wave results especially from the densification in propagation direction of the porous sample, which highly affect the decoupling process between the fluid and solid phase and is not considered in the conventional Biot theory. Figures 6.4 (C.) and 6.5 (C.) demonstrate that the P2-wave exhibits a smaller amplitude compared to the fast $\mathrm{P} 1$-wave. The relative motion between the solid and fluid phase, required for the propagation of the fast P2-wave, leads to higher energy dissipation and thus to smaller amplitudes. 


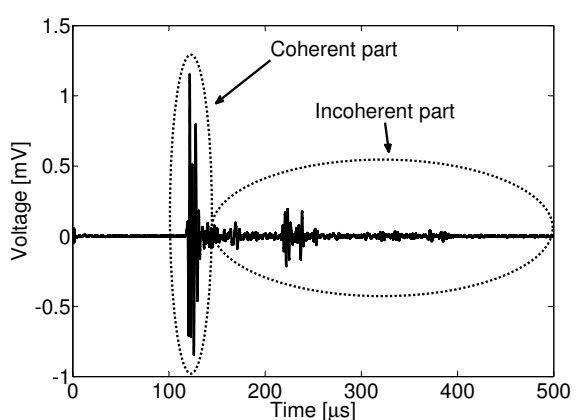

A.)

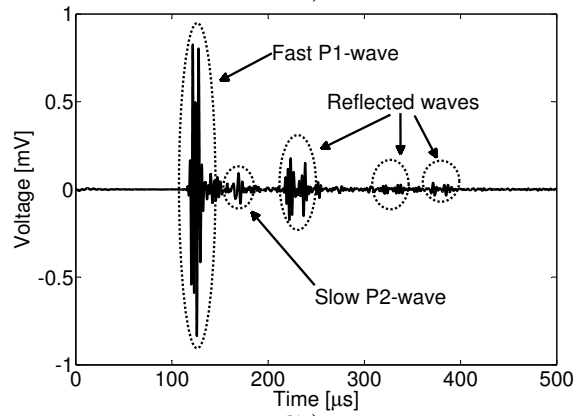

C.)

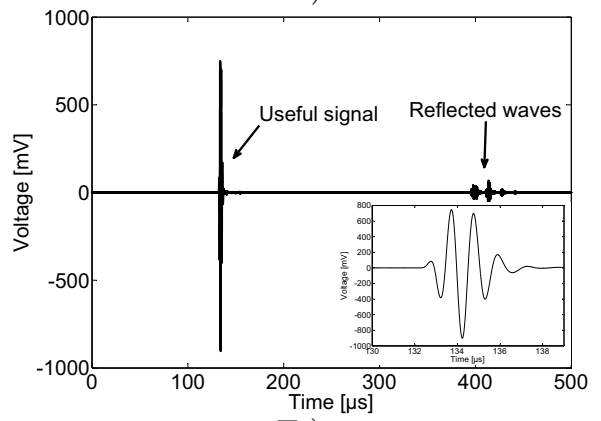

E.)

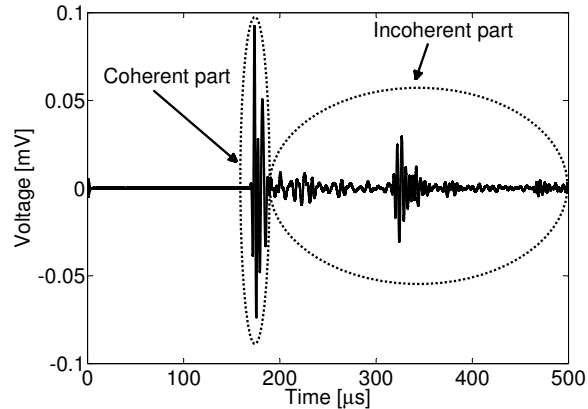

B.)

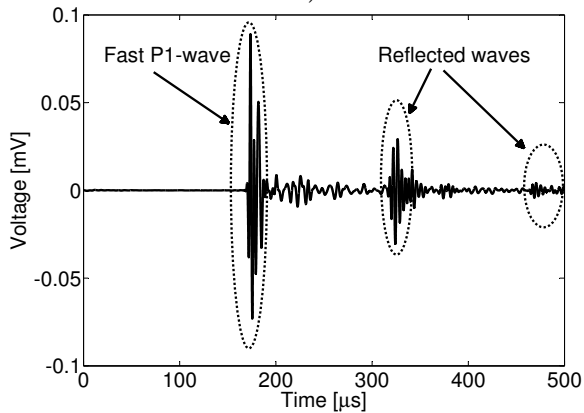

D.)

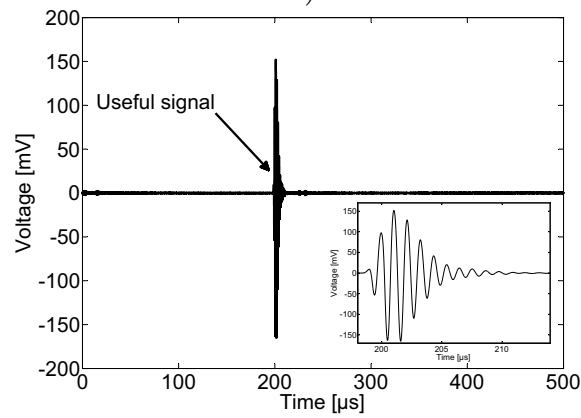

F.)

Figure 6.4: Received time signals of the water- and oil-saturated porous sintered sample in raw state (A.-B.) and after lowpass-filtering (C.-D.). For comparison the received signals of the reference measurements in water (E.) and silicone oil (F) are shown below. The insets in (E., F.) show an enlargement of the transmitted useful signals determined from the reference measurements. A 1cycle-sinus-burst-signal at $1 \mathrm{MHz}$ is used as electrical input signal. 
Note that the higher attenuation and even the hindrance of the generation of the slow P2-wave can also be attributed to underlying microstructural features of the investigated sample. The investigated sample shows a relatively high tortuosity of 1.46, which impedes the decoupling of the fluid and solid phase, but the most important point is the inhomogeneity in the direction of wave propagation. The investigated sample features an increasing density in propagation direction, which leads to a porosity and permeability gradients along the sample length. The densification along the sample length impede the fluid flow along the pore channels, so that the slow P2-wave cannot propagate. In such "layered" systems the coupling mechanism between fluid and solid phase is further enhanced and the propagation of the slow P2 wave is hindered. Sound propagation perpendicular to the density gradient might eventually lead to an increase of the amplitude of the slow P2-wave.

Hosokava et al. [85] have investigated the sound propagation through bovine cancellous bone and found out that the presence of the slow $\mathrm{P} 2$-wave is highly dependent on the propagation direction of the ultrasound wave with respect to the main direction of alignment of the trabeculae, which represents the porous bone matrix within the cancellous bone and is aligned towards the mechanical load distribution that a bone experiences [117]. These results were experimentally confirmed in human and bovine cancellous bones and the results were examined under the consideration of a stratified model [88, 107, 134].

Furthermore, Johnson et al. [94] have investigated the ultrasound propagation through water-saturated unconsolidated and slightly fused glass beads and reported that the slow P2-wave could only be observed, if the viscous skin depth is considerably less than the characteristic pore size. In this respect, he has introduced a second limit, named the viscous frequency

$$
f_{v}=\frac{4 \eta^{f R}}{\pi \rho^{f R} d_{p t}^{2} \zeta^{2}},
$$

where $\zeta$ is an empirically determined dimensionless constant of the order of 0.01 and $d_{p t}$ is a characteristic pore size. The coupling between the pore fluid and solid matrix is especially controlled at the narrowest place of the pore space, namely the pore throat. Using the mean pore throat diameter determined from the $\mu \mathrm{CT}$ data as the characteristic pore size leads to a viscous frequency of $f_{v}=9.27 \mathrm{MHz}$ for the silicone oil-saturated sintered sample, which is considerably higher compared to the frequency of the water-saturated sample of $f_{v}=0.46 \mathrm{MHz}$, which lies within the frequency range of the received ultrasound signals and ensures the propagation of the slow P2-wave.

The power spectra of the detected fast and slow P-waves are shown in Figure 6.6 (A.) and (C.). For a better comparability, the power spectra are normalized 


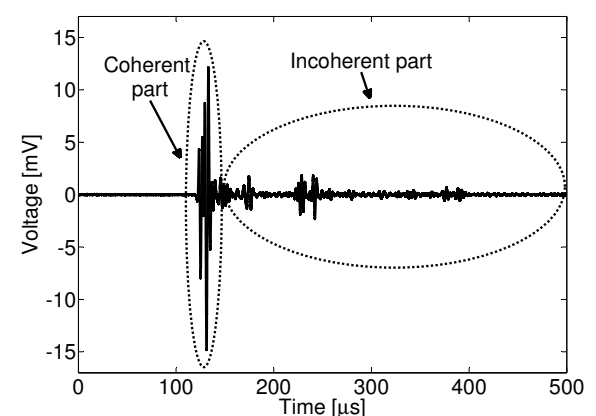

A.)

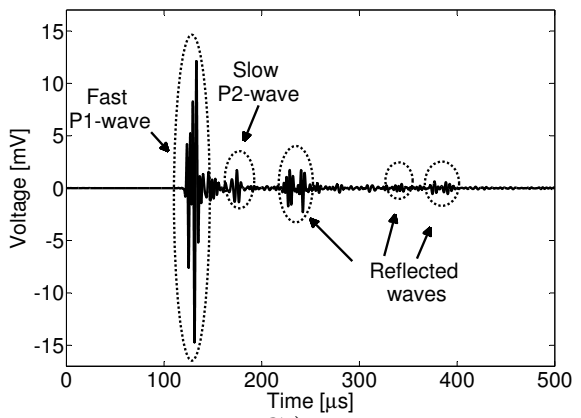

C.)

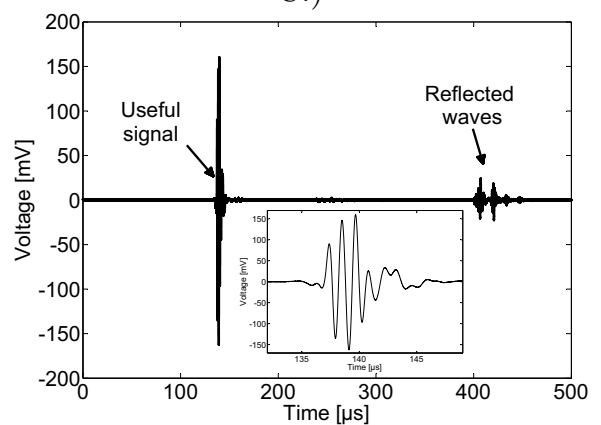

E.)

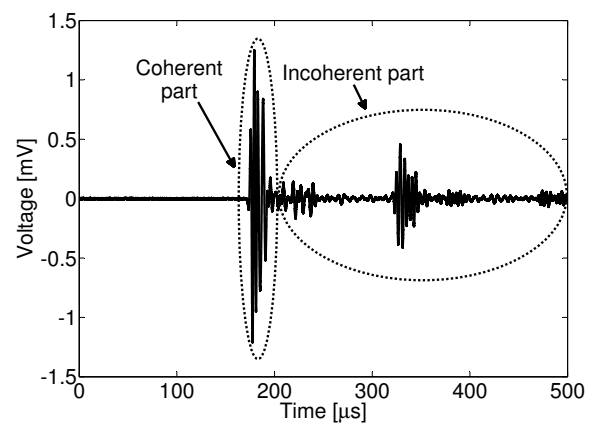

B.)

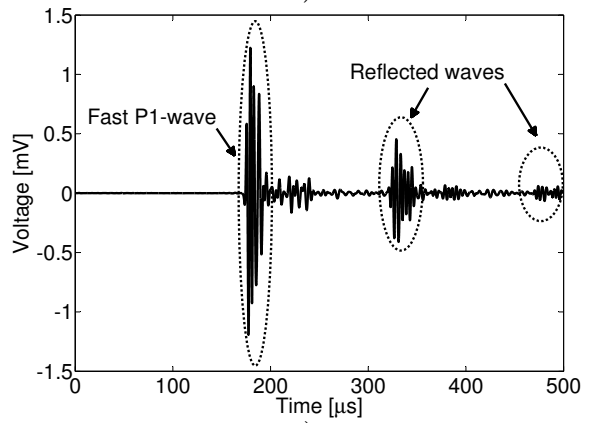

D.)

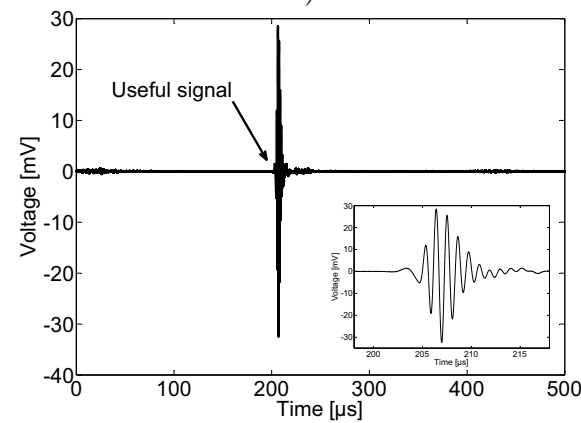

F.)

Figure 6.5: Received time signals of the water- and oil-saturated porous sintered sample in raw state (A.-B.) and after lowpass-filtering (C.-D.). For comparison the received signals of the reference measurements in water (E.) and silicone oil (F) are shown below. The insets in (E., F.) show an enlargement of the transmitted useful signals determined from the reference measurements. A 1cycle-Ricker-wavelet at $0.1 \mathrm{MHz}$ is used as electrical input signal. 


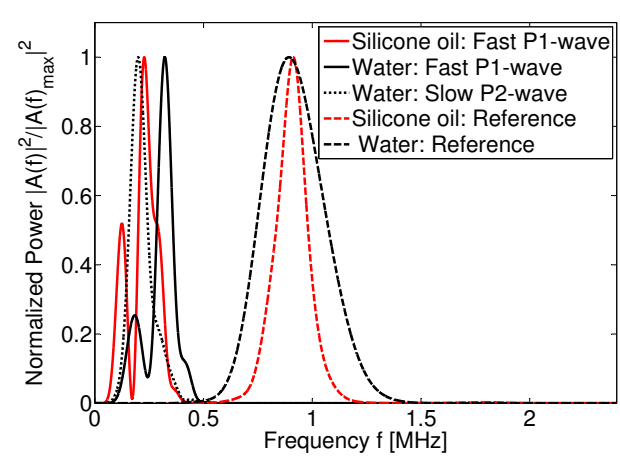

A.)

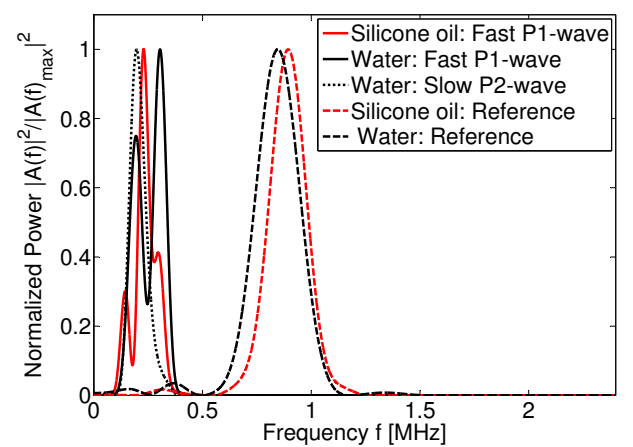

C.)

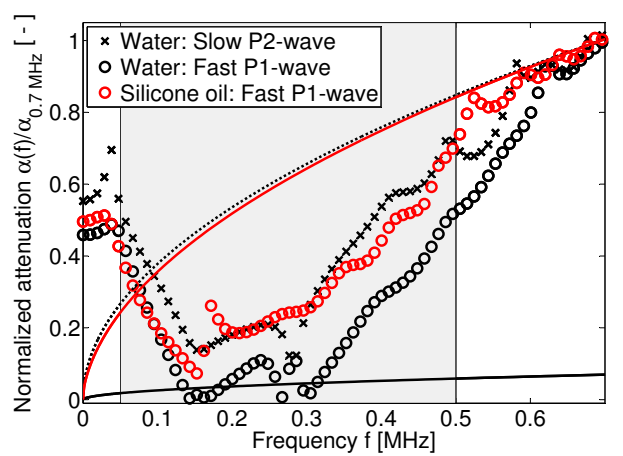

B.)

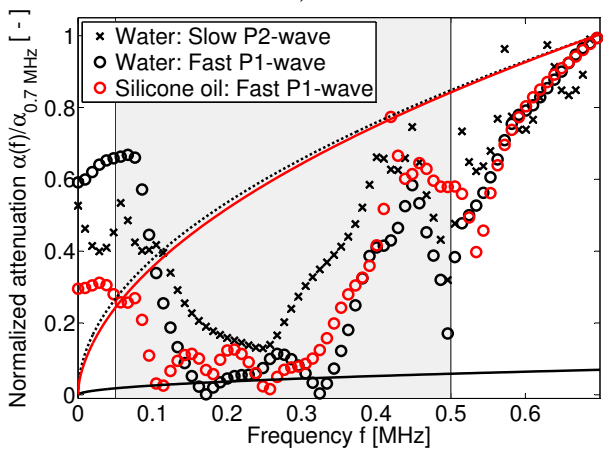

D.)

Figure 6.6: Illustration of normalized power spectra (A., C.) and normalized attenuations (B., D.) of the detected waves. The theoretical predictions of the attenuations of the detected waves are represented by solid and dotted lines (B., D.). The piezo-electric transmitter was excited with a 1-cycle-sinus-burst-signal at $1 \mathrm{MHz}$ (A., B.) or a 1-cycle-Ricker wavelet at $0.1 \mathrm{MHz}$ (C., D.). 
to their maximum values. It can be seen that the peaks are shifted to lower frequencies compared to the power spectra of the reference measurements. The high-frequency components of the incident ultrasonic wave is either filtered out by the sintered sample or converted to high-frequency (multiply-) scattered waves. The power spectrum obtained from the reference measurement in silicone oil is narrower compared to the power spectrum of the water measurement due to the additional cycles of the useful signal, although the same input signals have been used, see insets of Figures 6.4 and 6.5 (E.-F.). Moreover, it can be seen that the frequency range of the detected slow $\mathrm{P} 2$-wave in water lies within the frequency range of the fast $\mathrm{P} 1$-wave. Both $\mathrm{P}$-waves propagate with approximately the same frequency through the porous sample, independent from the used electrical input signal. Furthermore, the power spectra of the detected fast P1-waves exhibit at least two distinct peaks for both saturation fluids, while the power spectra of the $\mathrm{P} 2$-wave is monomodal.

The corresponding frequency-dependent attenuation coefficients $\alpha(f)$ at different excitation signals are presented in Figure 6.6 (B.) and (D.). The frequencydependent attenuation coefficients $\alpha(f)$ were determined form the amplitude spectra of the reference and sample measurement according to

$$
\alpha(f)=\ln \left(\frac{\left|A^{\mathrm{ref}}(f)\right|}{|A(f)|}\right) .
$$

For a better comparability between theoretical and experimental results the attenuation coefficients are normalized with the value at $0.7 \mathrm{MHz}$. The measured and predicted attenuation coefficients differ considerably and the Biot theory is insufficient to describe to attenuation mechanism of the detected $\mathrm{P}$-waves in the fluid-saturated porous sintered glass bead system. The different excitation signals, which result in different frequency-dependent attenuation coefficients, are not taken into account in the classical Biot theory.

\subsection{Conclusion}

In summary, we can state that the classical biphase Biot-theory can be used to identify the detected waves in ultrasonic measurements of water- or silicone oilsaturated sintered glass bead packings.

We have demonstrated that the saturating fluid plays a very important role in the presence of the slow P2-wave in ultrasonic measurements. While the slow-P2 wave in the water measurements could be clearly detected, the slow P2-wave in oil measurements could not be generated due to the higher viscous coupling and attenuation properties of the used silicone oil.

While the measured TOFs of the detected fast and slow P-waves are reflected 
properly by the Biot theory, the measured frequency-dependent attenuation coefficients differ considerably from the predicted values. Therefore, for a proper description of the dispersion properties (attenuation, phase velocities) a more sophisticated model is required, which takes the specific microstructural features, for instance the density gradient of the sintered sample in propagation direction of the ultrasonic waves into account. Ultrasound measurements perpendicular to the densification direction may eventually lead to an increase of the amplitude of the detected P2-wave or even to the presence of slow Biot-wave in siliconeoil-saturated sample, since the slow wave mostly occurs in directions where fluid flow is easily possible along the pore channels [105].

Future work should therefore include the investigation of ultrasound propagation at arbitrary angles of incidence on the sample surface. Moreover, a proper sintering method (rotational sintering) is required in order to produce more homogeneous samples. This will increase the probability for the detection of the slow P2-wave, since for it's generation a relative motion between the fluid and the solid is required. In homogeneous samples the relative motion between both constituents is easier than in samples with local porosity gradients.

\subsection{Acknowledgement}

We acknowledge support from the research program of the Stichting voor Fundamenteel Onderzoek der Materie (FOM), which is financially supported by the "Nederlandse Organisatie voor Wetenschappelijk Onderzoek" (NWO), (project number: 09iPOG14-2). 


\section{CHAPTER 7}

\section{The Effect of Magnetic Field on Wave Propagation in MRF-Saturated Porous Media}

In this paper, we present an experimental study of ultrasonic wave propagation in a porous sintered glass bead sample, which is saturated with a magneto-rheological fluid (MRF). The ultrasonic wave propagation in the MRF-saturated sample is studied at different magnetic strengths. For this purpose, a new-innovative measuring cell is designed and presented. In the ultrasound experiments, the transmission method is used to determine frequency-dependent dispersion properties (specific attenuation, intrinsic attenuation, phase velocity) of the MRF-saturated sample. Different excitation signals (sinus burst signals and Ricker wavelets)are used to study the ultrasound propagation through the MRF-saturated sample. The sample measurements are compared with reference measurements performed in the $M R F$, where no porous sample is placed between the transducers. In this study, we focus mainly on the investigation of the coherent deterministic pulse. A significant decrease of the amplitude of the received coherent pulse and a clear frequency shift of the peaks in the power spectra towards higher frequencies could be verified from the sample measurements with increasing magnetic strength. The reference measurements showed an increasing trend of the amplitude of the coherent pulse and the peaks of the power spectra occurred at the same frequency, when the MRF was subjected to a magnetic field. Moreover, the reference measurements showed 
a time shift of the coherent pulse whereas the sample measurements remained unchanged in terms of arrival times, when an external magnetic field is generated. We have demonstrated that dispersion properties of the MRF-saturated sample can be significantly influenced when a magnetic field is applied and that this effect is depends on the electrical input signal, with which the acoustic transmitter is briefly stimulated. The decay of the amplitude of the coherent wave with increasing magnetic strength transmitted through the MRF-saturated sample is also confirmed in the frequency-dependent attenuation coefficients. Furthermore, we have demonstrated the lowpass filtering effect of the used MRF. The cut-off frequency is dependent on the excitation signal and lied between 0.4 and $0.5 \mathrm{MHz}$. The lowpass filtering effect could be further enhanced when additionally a porous sample was positioned between the transducers.

\subsection{Introduction}

The study of ultrasonic wave propagation in fluid-filled porous materials has a long history and a crucial importance in many research fields. For instance, ultrasonic methods can be used to monitor the bone status $[74,83,84,99,106$, $132,133,185]$ or to increase the recovery of the oil from rock reservoirs $[7,12,124]$. In Enhanced Oil Recovery methods (EOR), ultrasound waves are applied to overcome the interfacial surface tension between oil and water, and subsequently reduce the capillary pressure in the pores of the rock $[18,89,123]$. The waveforms applied at specific frequencies, amplitudes and phases generate a second sound wave, often referred as slow P2- or Biot wave [24, 25, 122, 123], which arises from the out-of-phase movement between the solid and fluid phase. This causes the pore fluids trapped in porous rock formation and the porous rock matrix to move in opposite directions. The generated slow Biot-wave can, for instance, be used to move the oil in the porous rock structure towards already existing flow paths (percolation path) and be recovered from the rock formation.

In order to further increase the oil production rate in porous rocks the use of nanoand micron sized particles is very promising in Enhanced Oil Recovery methods, since they can accumulate at oil-water interfaces and additionally lower surface tension in similar fashion as surfactants $[22,23]$. Here, magnetic particles, as an example, can be traced or even guided when a magnetic field is generated outside the porous medium. In this respect, magneto-rheological fluids (MRF), which are magnetizable micron-sized particles suspended in a carrying fluid (mostly a hydrocarbon-based solvent) constitute promising candidates to be used in EOR methods. MRFs greatly change their apparent viscosity, when subjected to a magnetic field, to become a viscoelastic solid [45]. Moreover, the yield stress 


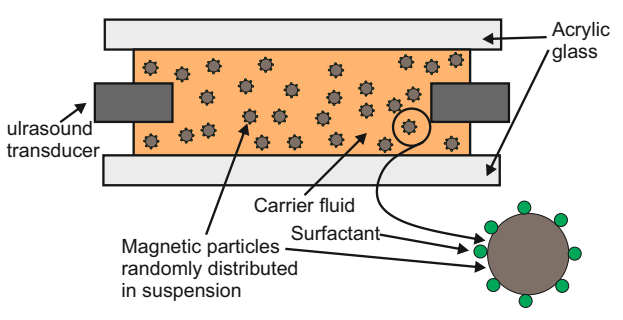

A.)

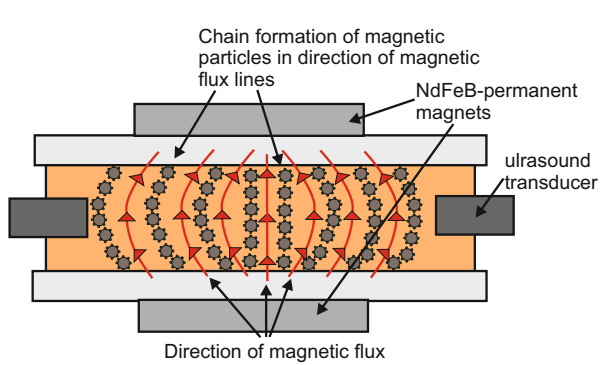

B.)

Figure 7.1: Illustration of MRF behavior, in the cases when no magnetic field is applied (A.) or a magnetic field (B.) is generated [141].

of the suspension can be controlled selectively and accurately by varying the magnetic strength [171]. Hereby the micro-sized particles align themselves along the magnetic field lines, see Figure 7.1, whereas under normal conditions, when no magnetic field is generated, the magnetizable particles are randomly distributed in the suspension.

Numerous studies addressing the rheological behavior of magneto-rheological fluids, which preferably used in dampers, brakes, hydraulic valves, clutches etc., exist already exist [37-39, 62, 64, 65, 97, 112, 142, 182]. However, their acoustical properties and behavior in a porous medium is rarely investigated and understood. Due to their aforementioned advantages MRFs can be used to study the internal physical interactions between the fluid and solid, which highly affect the acoustic properties of the fluid-filled materials. The properties of the pore fluid, such as the viscosity, density and bulk modulus can be interactively changed during ultrasound experiments and thus the internal physical coupling and decoupling processes of the detected waves can be precisely changed with the applied magnetic strength.

Therefore, in this paper, we present broadband ultrasonic experiments on a MRFsaturated porous sintered glass bead sample under external generated magnetic field. Compared to many natural and artificial porous materials, the used sintered sample has a simple and repeatable pore structure. The ultrasound results at different applied magnetic strengths are compared with ultrasound measurements at normal conditions when the MRF-saturated sample is not subjected to an external magnetic field. The spectral ratio technique, which is applied to determine the dispersion properties of the MRF-saturated sample at different applied magnetic strengths, is presented in chapter 2. The experimental procedure and measuring cell including the magnets used for the ultrasound experiments 
are described in chapter 3 . The results obtained from the transmission signals are presented in the following chapter 4 . The main findings from the ultrasound experiments are concluded in the final chapter.

\subsection{Background theory and evaluation algorithm}

In many research areas, such as geophysics or medical engineering, the spectral ratio technique is often applied in ultrasound investigations to examine the frequency-dependent dispersion properties of porous rocks or bones, which are often saturated with different fluids. The spectral ratio technique has found the be a reliable method and therefore found the broadest acceptance [107, 134, 179]. In this method, a reference signal is recorded with no porous sample in the path and the other is recorded with the porous sample in between the transducers [198]. Therefore, this method is also often referred as the substitution method [107]. The signal transmitted through the fluid-saturated porous medium is compared with the signal transmitted through the reference medium with specific attenuation $\alpha_{\text {ref }}$ and speed of sound $c_{\text {ref }}$.

In addition to the frequency-dependent phase velocity, this method enables the determination of the so-called frequency-dependent quality factor $Q$, or it's inverse the specific attenuation $Q^{-1}$, which quantifies the intrinsic attenuation of seismic energy [72]. According to [179] the quality factor is defined as the fractional energy loss per cycle experienced by a ultrasonic wave. The quality factor is usually expressed as

$$
Q=\frac{\pi f}{\hat{\alpha}(f) c(f)}
$$

where $f, \hat{\alpha}(f)$ and $c(f)$ are the frequency, the frequency-dependent intrinsic attenuation normalized to the sample length and phase velocity of the ultrasound wave [125]. Depending on the propagation medium and saturation degree of the pore fluid, the quality factor can considerably differ from sample to sample. However, the behavior of the frequency-dependent quality factor in MRF-saturated porous samples under different magnetic strength has not been investigated yet. The quality factor in Equation (7.2) determined from ultrasound experiments is an effective value, which is controlled by intrinsic effects of the investigated sample and also by additional (apparent) attenuation processes, such as scattering from inclusion, heterogeneity, the effect of interface losses, etc. [72, 107]:

$$
\frac{1}{Q_{\mathrm{eff}}}=\frac{1}{Q_{\mathrm{int}}}+\frac{1}{Q_{\mathrm{add}}}
$$

Following the fundamental principles of ultrasonic wave attenuation and dispersion, we can describe the amplitude spectrum of the received waveform that has 
propagated through the reference medium (MRF) as follows

$$
A_{\text {ref }}(f)=A_{0}(f) A_{\text {ref }}^{d}(f)
$$

where $A_{0}(f)$ is the amplitude spectrum of the electrical input signal and the transfer functions of the receiving and transmitting transducers and electronics. $A_{\text {ref }}^{d}(f)$ is the transfer function which characterizes the effect of ultrasound propagation for the MRF path only [43]. In this study, sinus-burst-signals and Ricker wavelets are used as electrical input signals.

According to [107] the amplitude spectrum of the received sample signal can be expressed as

$$
A(f)=A_{0}(f) T(f) A_{d}(f) e^{-\hat{\alpha}(f) l}
$$

where $A_{d}(f) e^{-\hat{\alpha}(f) l}$ characterizes the transfer function of the ultrasound wave, when additionally a porous sample is positioned between the transducers. The term $e^{-\hat{\alpha}(f) l}$ describes the attenuation caused by the porous sample with length l. $T(f)$ represents the transmission coefficient for the reference fluid-sample and sample-reference fluid interfaces. The diffraction transfer functions in Equations (7.3) and (7.4) are represented by $A_{d}(f)$ and $A_{\text {ref }}^{d}(f)$.

The frequency-dependent apparent attenuation $\bar{\alpha}(f)$ is usually defined on the logarithmic scale as

$$
\bar{\alpha}(f) l=\ln \left(\frac{\left|A_{\mathrm{ref}}(f)\right|}{|A(f)|}\right) .
$$

By inserting Equations (7.3) and (7.4) in Equation (7.5) the apparent attenuation can be expressed as

$$
\bar{\alpha}(f) l=\hat{\alpha}(f) l+\ln \left(\frac{\left|A_{\mathrm{ref}}^{d}(f)\right|}{\left|A_{d}(f)\right|}\right)-\ln (|T|),
$$

where $\hat{\alpha}(f) l$ represents the actual intrinsic attenuation of the signal transmitted through the MRF-saturated sample. In the considered frequency range of 0.1$0.4 \mathrm{MHz}$ for the ultrasound experiments, the slope of the determined apparent attenuation $\hat{\alpha}(f) l$ yields the quality factor $Q$. In the some cases, for instance in water-bone-systems $[43,107]$, it is assumed that the

- the effect of diffraction $\ln \left(\frac{\left|A_{\text {ref }}(f)\right|}{|A(f)|}\right)$ is negligible small [43],

- the transmission losses $\ln (|T|)$ are frequency-independent and do not affect the slope of the attenuation $[43,107]$,

- phase cancellation effects can be neglected, which occurs especially when the fast and slow P-waves overlap in time [13, 15, 120]. 
The TOFs of the reference and sample measurements can be calculated [107] in the time domain as

$$
\begin{aligned}
\text { reference material : } & T O F_{\mathrm{ref}} & =\frac{L}{c_{\mathrm{ref}}} \\
\text { reference and sample material : } & T O F & =\frac{L-l}{c_{\mathrm{ref}}}+\frac{L}{c} .
\end{aligned}
$$

The corresponding sound velocity of the MRF-saturated sample is determined from the time difference of the sample and reference measurements [73], given in Equations (7.7) and (7.8), as

$$
c=\frac{l}{\frac{l}{c_{\mathrm{ref}}}-\Delta T O F},
$$

where the time difference between the sample and reference measurement is defined as $\triangle T O F=T O F_{\text {ref }}-T O F$. For the determination of the frequencydependent phase velocities, we use the phase spectra of the sample and reference measurement

$$
\begin{aligned}
\Delta \phi(f) & =\phi(f)-\phi_{\mathrm{ref}}(f)=\arctan \left(\frac{\operatorname{Im}(A(f))}{\operatorname{Re}(A(f))}\right)-\arctan \left(\frac{\operatorname{Im}\left(A_{\mathrm{ref}}(f)\right)}{\operatorname{Re}\left(A_{\mathrm{ref}}(f)\right)}\right) \\
& =2 \pi f l\left(\frac{1}{c_{\mathrm{ref}}}-\frac{1}{c(f)}\right)
\end{aligned}
$$

Reformulating Equation (7.10) yields the frequency-dependent phase velocities

$$
c(f)=\frac{l}{\frac{l}{c_{\text {ref }}}-\frac{\Delta \phi_{u}(f)}{2 \pi f}},
$$

where the time difference in Equation (7.9) is expressed in terms of the frequency $f$ and phase difference $\Delta \phi_{u}(f) . \Delta \phi_{u}(f)$ denotes the unwrapped phase difference, where the discontinuities of the arc-tangent functions in the calculations of the phase spectra are removed. It should be noticed, that the short ultrasound pulses used in this experiment (1-cycle-Ricker and 1-cycle-sinus) show a broad frequency (0.01-2.0 MHz), and each frequency present in the signal react differently to the structure of the used sintered glass bead sample and MRF in terms of attenuation and phase velocity.

Figure 7.2 shows flow chart for the determination of the dispersion properties according to spectral ratio method. First, the time signals obtained from reference and sample measurements are acquired. So-called time windows are applied to isolate the useful signal from the remaining part of the signal. The isolated 


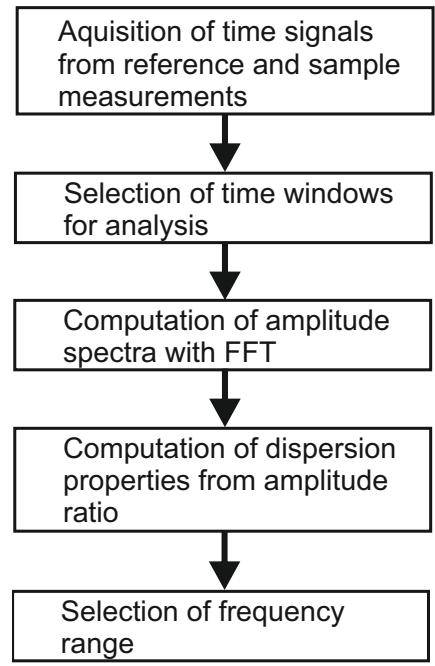

Figure 7.2: Flow chart for determination dispersion properties (i.e. $\hat{\alpha}(f), Q(f)$, $c(f))$ according to the spectral ratio method.

signal parts are transformed via FFT into the frequency domain to determine the amplitude spectra. The frequency-dependent dispersion properties are determined from the amplitude ratio of the sample and reference measurement. In the final step, the relevant frequency range of the determined dispersion parameters is identified.

\subsection{Experiments}

\subsubsection{Sintered specimen and MRF}

The sintered glass bead sample used in this study is developed in a special sintering set-up, which is described in [69, 71]. Figure 7.3 (A.) depicts a photograph of the investigated sample, which has a bulk diameter of $30 \mathrm{~mm}$ and sample length of $50 \mathrm{~mm}$. The solid matrix is composed of sintered glass beads with particle diameters from 1.0 to $1.2 \mathrm{~mm}$. Compared with many natural and artificial porous materials, the sintered sample shows a simple and repeatable pore geometry. Figure 7.3 (B.,C.) shows the volume-rendered solid matrix and the corresponding pore structure determined from $\mu \mathrm{XRCT}$ data of the investigated sample. A detailed analysis of the solid matrix and pore space is described in chapters 2 and 


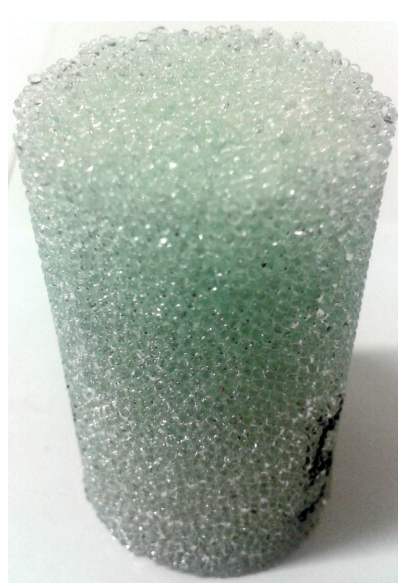

A.)

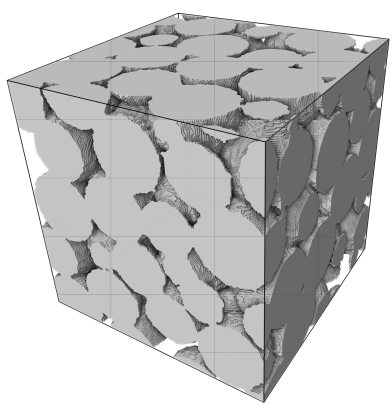

B.)

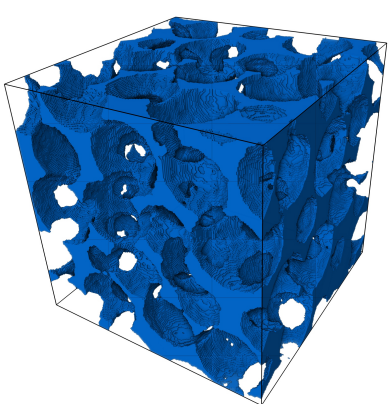

C.)

Figure 7.3: Photograph of the used porous sintered glass bead sample (A.) and $\mu \mathrm{CT}$ image of the solid matrix (B.) and corresponding pore structure (C.).

4 .

The photographs in Figure 7.4 shows the commercial available magneto-rheological fluid (MRF-140CG, Lord MR), which is used for the ultrasound experiments, at different states. The used MRF-140CG is a dark gray suspension with magnetizable, micron-sized particles in a silicone oil-based carrying fluid [3]. To decrease the rate of ferro particle setting and guaranty a stable suspension, surfactant are added to the used MRF-140CG. As can be seen from pictures in Figure 7.4 (A.) the used MRF-140CG allows free movement in the absence of a magnetic field, and when a magnetic field is applied, the ferro particles align themselves with the direction of the applied magnetic field in chain-like fashion [3], see Figure 7.4 (B.). In Figure 7.4 (B.) a NeFeB-permanent magnet is used to restrict the fluid movement and generate visco-elastic solid.

Further properties of the used MRF-140CG and the produced sintered glass bead sample are listed in Table 7.1.

\subsubsection{Experimental procedure}

In this study, ultrasonic measurements are made according to the pulse transmission technique consisting two identical immersion broadband transducers with 


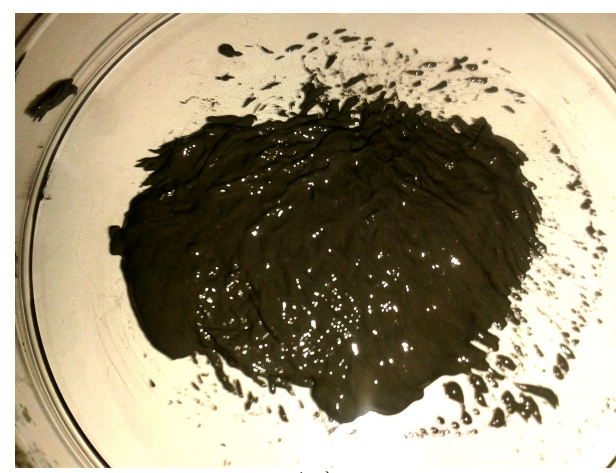

A.)

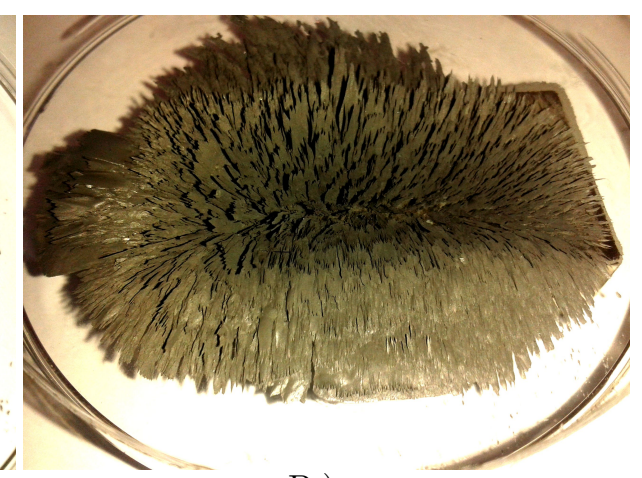

B.)

Figure 7.4: Photographs of the used MRF 140CG, when no magnetic field is applied (A.) and a magnetic field is generated by using a NeFeB-permanent magnet (B.).

Table 7.1: Material parameters of the used ferro fluid and sintered glass bead sample.

\begin{tabular}{|c|c|c|}
\hline Parameter & Unit & Magneto-rheological fluid \\
\hline Product name $[A]$ & {$[-]$} & MRF-140CG \\
\hline Carrying fluid $[A]$ & {$[-]$} & synthetic hydrocarbon base and decanedioic acid \\
\hline $\operatorname{Viscosity}[A]$ at $40^{\circ} \eta^{f R}$ & {$[\mathrm{Pas}]$} & $0.280 \pm 0.070$ \\
\hline Density $[A]$ & {$\left[\mathrm{g} / \mathrm{cm}^{3}\right]$} & $3.54-3.74$ \\
\hline Solid content by weight ${ }^{[1]}$ & [\%] & 85.44 \\
\hline Flash Point $[A]$ & {$\left[{ }^{\circ} \mathrm{C}\right]$} & 150 \\
\hline Operating temperature ${ }^{[A]}$ & {$\left[{ }^{\circ} \mathrm{C}\right]$} & -40 to +130 \\
\hline Particle size of Fe-particles ${ }^{[B]}$ & {$[\mu \mathrm{m}]$} & $0.88-4.03$ \\
\hline Parameter & Unit & Sintered glass bead sample \\
\hline Tortuosity factor ${ }^{[C]}$ & {$[-]$} & 1.54 \\
\hline Effect. porosity $[D]$ & {$[\%]$} & 31.84 \\
\hline Effect. permeability $[E]$ & {$\left[\mathrm{m}^{2}\right]$} & $4.58 \times 10^{-10}$ \\
\hline Particle diameter of beads & {$[\mathrm{mm}]$} & $1.0-1.2$ \\
\hline
\end{tabular}

$[A]$ : manufacturer information, see Ref. [3]

$[B]$ : see Ref. [11]

${ }^{[C]}$ : Obtained from $1024 \times 1024 \times 2048$ cuboids extracted from $\mu \mathrm{CT}$ data

$[D]$ : Obtained from bulk density and volume

$[E]$ : determined from stationary permeability experiments 
central frequencies of $2.25 \mathrm{MHz}$ (Olympus, Panametrics NDT, V306-SU). The piezo-electric transducers are aligned contrarily and mounted in specially developed thin-walled measuring cell made of acrylic glass at fixed distance of $84 \mathrm{~mm}$, cf. Figure 7.6. The electrical pulses for the broadband transmitter were generated by an arbitrary function generator (Tektronix, AFG 3101, 1 GS/s, $100 \mathrm{MHz}$ ) and power-amplified with $55 \mathrm{~dB}$ (RF Power Amplifier, model E\&I 1040L, $10 \mathrm{kHz}$ $5 \mathrm{MHz}, 400 \mathrm{~W}$ ). Sinus-burst signals and Ricker wavelets at different frequencies are used as electrical input signal to simulate the piezo-electric acoustic transmitter. The Ricker wavelet is the negative normalized second derivative of a Gaussian function and is often referred as Mexican hat wavelet due to it's shape. The amplitude of the Ricker wavelet is defined in the time domain as

$$
A(t)=\frac{2}{\sqrt{3 \sigma} \pi^{1 / 4}}\left(1-\frac{t^{2}}{\sigma}\right) e^{\frac{-t^{2}}{2 \sigma^{2}}},
$$

where $t$ and $\sigma$ represent the time and duration of the wavelet signal [63]. The Ricker wavelet represents theoretically a solution of the Stokes differential equation, which takes into account the effects of Newtonian viscosity. Therefore, it is often applied as model seismic wavelet which propagates through a viscoelastic homogeneous porous media [183]. For the ultrasound measurement a maximum of 2 cuboid-shaped permanent magnets are used. The residual magnetic strength for each of the used permanent magnets is $B=1.26-1.29$ T. Further characteristic parameters of the NeFeB-magnets are listed in Table 7.2. In order to investigate the influence of an magnetic field on the ultrasound experiments, the NeFeB-magnets are attached at the top or/and bottom measuring cell, where the thickness of the plates made of acrylic glass is only $3.35 \mathrm{~mm}$, see Figure 7.6.

The signal from the receiving transducer is first pre-amplified with 20 or $40 \mathrm{~dB}$ using a spike impulser (Olympus, Panametrics NDT, model 5052PR) and finally fed to a $200 \mathrm{MHz}$ digital storage oscilloscope (LeCroy 24Xs-A, 2.5 GS/s), which is interfaced to a PC. In order to minimize the noise level, the received signals are averaged over 512 acquisitions. The synchronization is achieved by triggering the oscilloscope from the output of the function generator. Prior to the ultrasound measurements, the measuring cell including porous sample is rinsed out with carbon dioxide to achieve an optimal saturation of the porous sample. The sintered specimen itself is coated with a latex membrane and fixed on a specially-developed sample holder, in order to prevent a sliding of the sample during saturation with the high viscous MRF. The sintered sample is positioned in the center of the measuring cell and the distance to both immersion transducers is fixed at $17 \mathrm{~mm}$. Figure 7.5 shows a scaled CAD drawing of the measuring cell, which was used to study the effect of magnetic field strengths on the wave propagation in MRF-saturated porous sintered glass bead sample. 


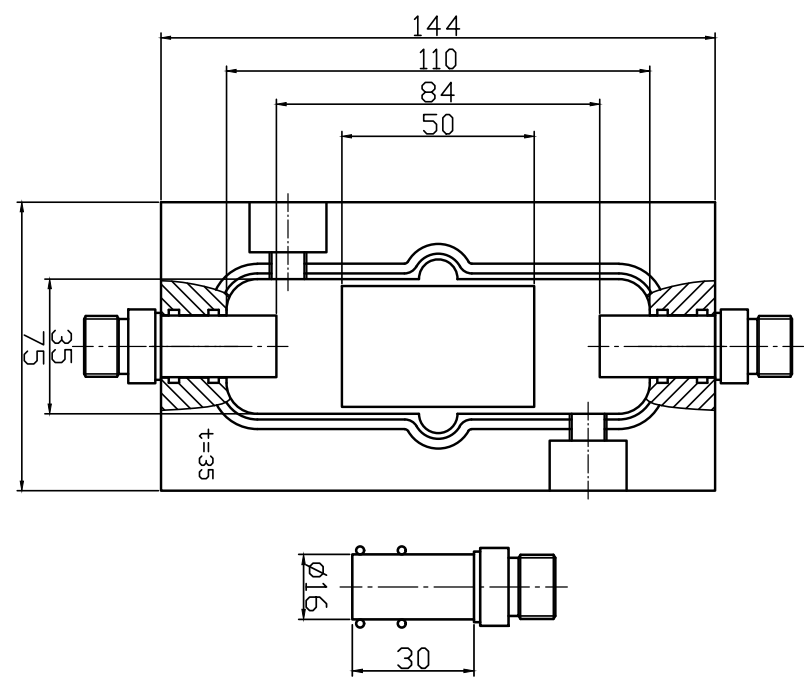

Figure 7.5: Scaled CAD drawing of the thin-walled measuring cell and the used broadband immersion transducers (central frequency $2.25 \mathrm{MHz}$, PanametriceNDT, V306-SU). Length informations are given in $\mathrm{mm}$.

The saturation of the sample is performed using a syringe pump, see Figure 7.6.

\subsection{Results and discussion}

\subsubsection{Time signals and power spectra}

The waveforms for the ultrasound pulse traveling through the MRF-saturated sample at different magnetic strength are shown in Figure 7.7 (B.). For comparison the MRF pulse received from ultrasound measurements without placing porous sample is shown in Figure 7.7 (A.). From Figures 7.7 (A.) and (B.), we can clearly see that the received time signals basically consist of a coherent deterministic pulse characterized by large amplitudes and an incoherent part, which arrives at certainly later times and show smaller amplitudes compared to the coherent pulse at the leading edge.

For the determinations of the power spectra and the dispersion properties only the coherent parts are considered. The coherent part is windowed to separate 


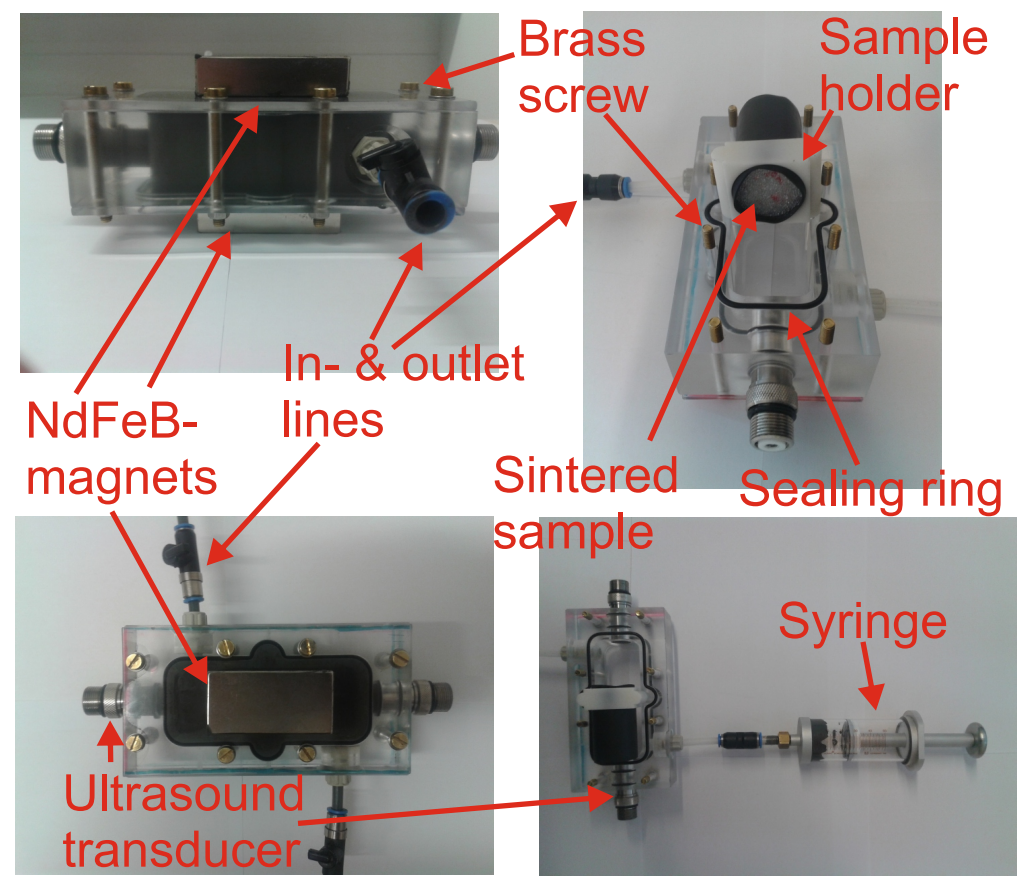

Figure 7.6: Photographs of the measuring cell made of acrylic glass and designed to investigate the influence of magnetic strength on the acoustical wave propagation of MRF-saturated porous samples.

Table 7.2: Material parameters of the used permanent magnets ${ }^{[1]}$.

\begin{tabular}{l|c} 
Chemical composition & NdFeB \\
Coating & Nickel $(\mathrm{Ni}-\mathrm{Cu}-\mathrm{Ni})$ \\
Volume $(\mathrm{L} \times \mathrm{W} \times \mathrm{H})$ Length $\left[\mathrm{mm}^{3}\right]$ & $60 \times 30 \times 15$ \\
Approx. magnetic strength $[\mathrm{N}]$ & 550 \\
Maximum working temperature $\left[{ }^{\circ} \mathrm{C}\right]$ & 80 \\
Weight $[\mathrm{g}]$ & 205.2 \\
Curie temperature $\left[{ }^{\circ} \mathrm{C}\right]$ & 310 \\
Residual magnetism $B[\mathrm{~T}]$ & $1.26-1.29$ \\
Coercive field strength $b_{H c}[\mathrm{kA} / \mathrm{m}]$ & $860-955$ \\
Coercive field strength $i_{c h}[\mathrm{kA} / \mathrm{m}]$ & $\geq 955$ \\
Energy product $(B \times H)_{\max }\left[\mathrm{kJ} / \mathrm{m}^{3}\right]$ & $303-318$ \\
\hline
\end{tabular}

[1] manufacturer information, see Ref. [5] 


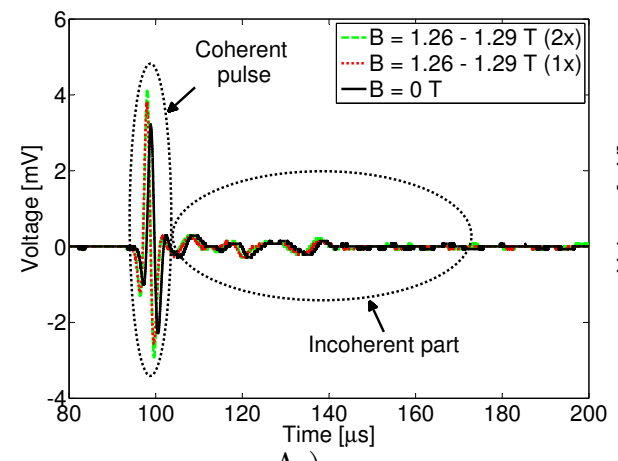

A.)

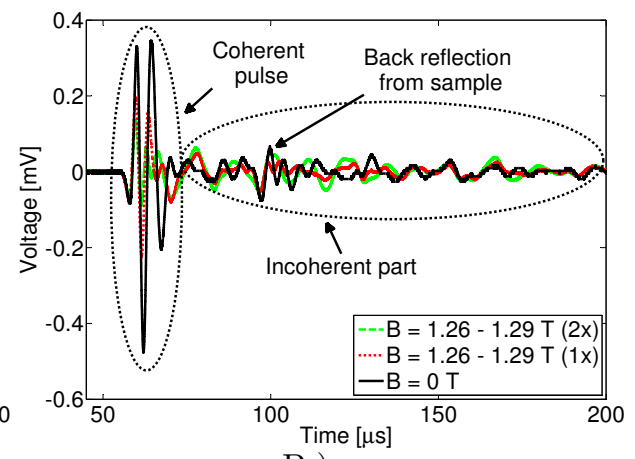

B.)

Figure 7.7: Received time signals of MRF only (A.) and MRF-saturated sintered glass bead sample (B.). The piezoelectric transmitter is excited with a sinus-burst-signal at $1 \mathrm{MHz}$. The coherent and incoherent parts are marked accordingly.

it from remaining part of the signal and subsequently the FFT is calculated. In general, the temporal widths of the time windows decrease with increasing frequency according to $T=2 \pi / \omega$. Figure 7.8 shows exemplary the raw signal and the isolated coherent part for the reference measurement performed in MRF only, as well as for the sample measurement, whereby the piezo-electric acoustic transmitter is excited with a sinus-burst-signal at $1 \mathrm{MHz}$.

Figure 7.9 illustrate the enlarged coherent parts of the received time signals (A.,C.) and the corresponding power spectra (B.,D.) of the reference and sample measurement at different magnetic strength $B$, when the transmitter was excited with a sinus-burst-signal at $1 \mathrm{MHz}$. The coherent part of the sample measurement corresponds to the fast P1-wave, which arises mainly from the inertial coupling between the pore fluid and solid matrix. The hydrodynamic coupling of the pore fluid on the solid frame is proportional to the relative velocity between the solid frame and fluid [91]. It can be clearly seen that the maximum peaks of the reference measurement increase with increasing magnetic strength, whereas in the case of the sample measurement the opposite effect occurs. The amplitudes of the coherent wave part of the sample measurements are more damped and decrease with increasing magnetic strength $B$ (or increasing number of used NdFeB-magnets), since the pore fluid behaves more stiffer with increasing magnetic strength. This indicates the low coupling mechanism between the both constituents. In contrast to this, the fast P-wave of the reference measurement 


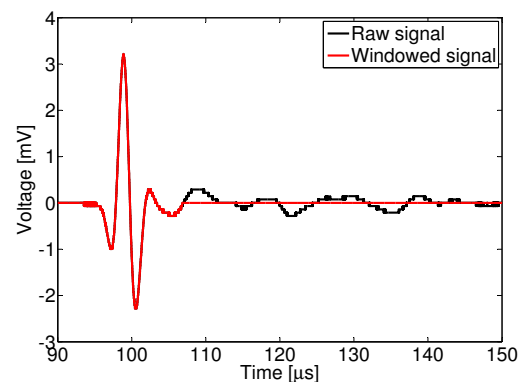

A.)

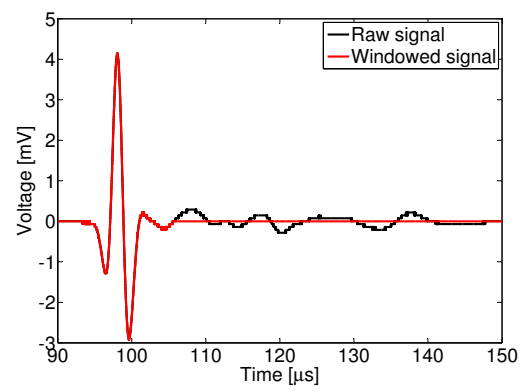

C.)

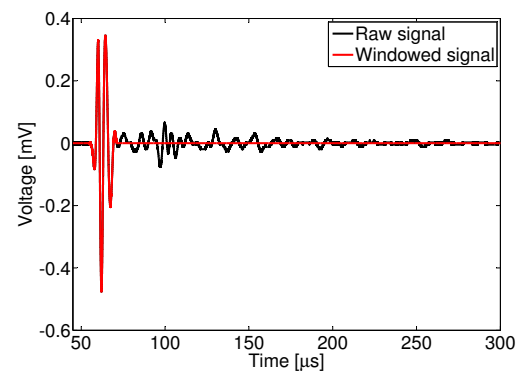

E.)

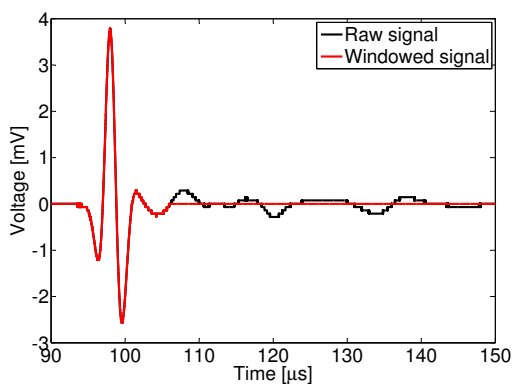

B.)

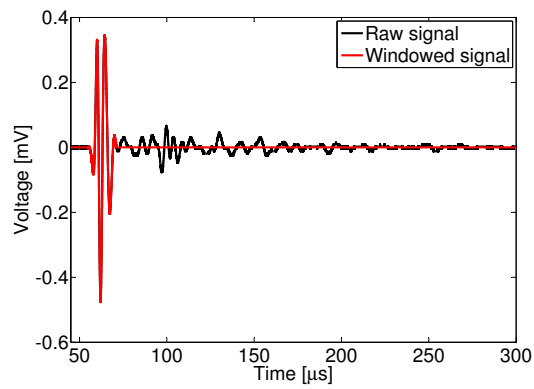

D.)

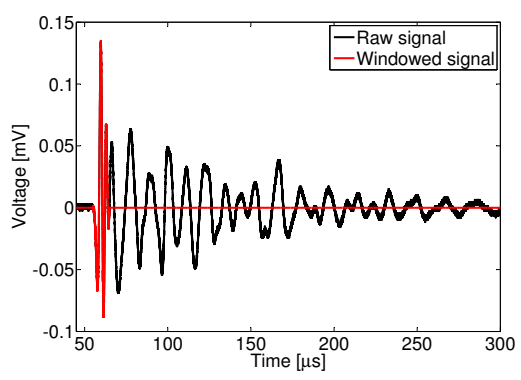

F.)

Figure 7.8: Examples of raw and windowed signals, when the acoustic transmitter is excited with a sinus-burst-signal at $1 \mathrm{MHz}$. 


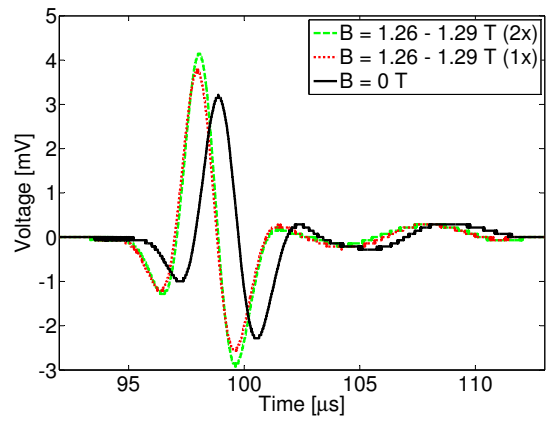

A.)

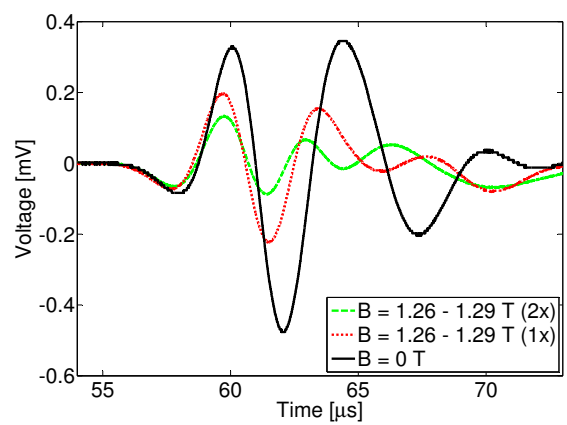

C.)

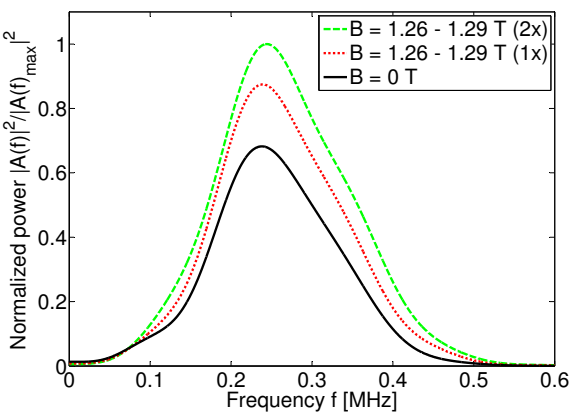

B.)

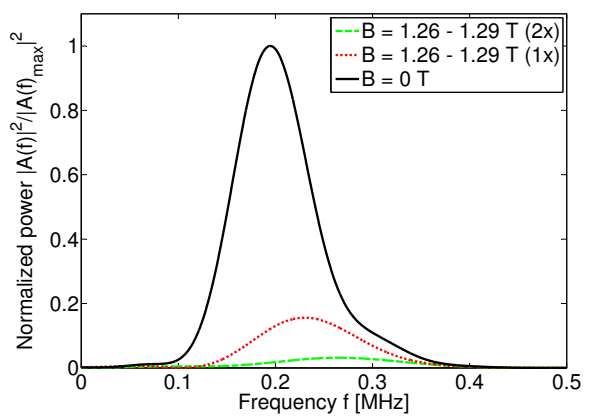

D.)

Figure 7.9: Coherent Part of the received time signals (A.,C.) transmitted through only MRF and MRF-saturated sintered sample with corresponding normalized power spectra (B.,D.). The piezo-electric acoustic transmitter is excited with a sinus-burst-signal at $1 \mathrm{MHz}$.

propagates faster and with higher intensity, when the MRF is subjected to a magnetic field due to chain forming of the iron particles suspended in the fluid. The amplitude change at different magnetic field strengths is also highlighted in the normalized power spectra Figure 7.9 (B.,D.).

For the reference measurement the TOF of the detected coherent wave decreases about $1.90 \mu \mathrm{s}$, when a magnetic field strength is increased from 0 to 1.26-1.29 T. For further increase in magnetic strength the TOF remains relatively unchanged. In contrast to that, the TOFs of the sample measurements remain relatively constant independent on the applied magnetic strength $B$. The TOFs and the corresponding Speed Of Sound (SOS) determined in the time domain are summarized 
Table 7.3: Experimental and theoretical determined TOFs and SOSs of fast P1wave of investigated sintered samples at a excitation frequency of $1 \mathrm{MHz}$.

\begin{tabular}{lcccc}
\hline Parameter & Unit & 0 Magnet & 1 Magnet & 2 Magnets \\
\hline \hline Magnetic strength $B$ & {$[\mathrm{~T}]$} & 0 & $1.26-1.29$ & $1.26-1.29$ \\
Time Of Flight $T O F_{\text {ref }}$ & {$[\mu \mathrm{s}]$} & 95.78 & 94.87 & 94.99 \\
Time Of Flight $T O F$ & {$[\mu \mathrm{s}]$} & 56.07 & 55.86 & 55.91 \\
Time difference $\triangle T O F$ & {$[\mu \mathrm{s}]$} & -39.71 & -39.01 & -39.08 \\
Speed Of Sound $c_{\text {ref }}$ & {$[\mathrm{m} / \mathrm{s}]$} & 877.01 & 885.42 & 884.30 \\
Speed Of Sound $c$ & {$[\mathrm{~m} / \mathrm{s}]$} & 2889.86 & 2863.65 & 2863.42 \\
\hline \hline
\end{tabular}

in Table 7.3. Moreover, it is remarkable that the peak obtained from the sample measurement is shifted to higher frequencies with increasing magnetic strength $B$, whereas the frequencies of the peaks for the reference measurement remain almost identical with changing magnetic strength $B$. Since the power spectra of the sample measurements lie within the frequency spectrum of the reference measurements, a linear wave conversion at boundary between the solid and fluid phase can be assumed. The almost same distributions for the power spectra are observed, when the frequency of the input waveform is changed to $2.25 \mathrm{MHz}$, which corresponds to central frequency of the used immersion transducers, cf Figure 7.10 .

The power spectra of the reference measurements in Figure 7.9 and 7.10 clearly demonstrate that the frequency spectrum is limited to approximately $0.5 \mathrm{MHz}$, although sinus-burst-signals with frequencies of 1.0 and $2.25 \mathrm{MHz}$ have been used. It proves that the high-frequency component $(f>0.5 \mathrm{~Hz})$ of the broadband input signal is filtered out through the MRF. The filter effect is further enhanced through the porous sintered sample. The cut-off frequency is lower, when additionally a porous sample is placed between the transducers and lies at approximately at $0.4 \mathrm{MHz}$.

Similar lowpass filter effects of the MRF and the sintered sample are also observed for different excitation signals at different frequencies. Figures 7.11 and 7.12 show the time signals and amplitude spectra, when the transmitter is excited with a Ricker wavelet at 0.2 and $1 \mathrm{MHz}$. The cut-off frequencies of the MRF and the porous sintered sample are lower compared to the results obtained from excitation with a sinus burst signal. The cut-off frequency lies at 0.4 and $0.32 \mathrm{MHz}$, respectively. It is also apparent from the power spectra, that for the reference measurements, the filter effect slightly decreases with increasing number of used 


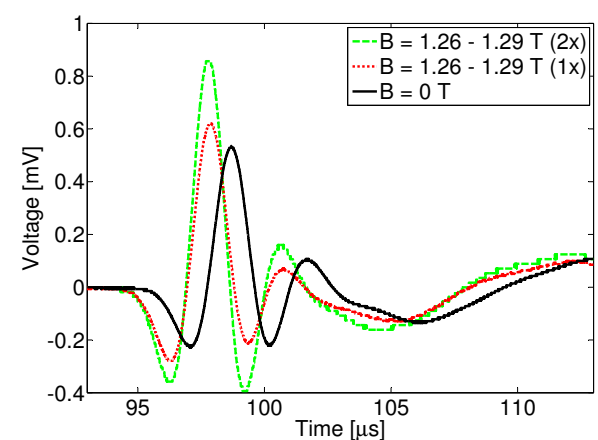

A.)

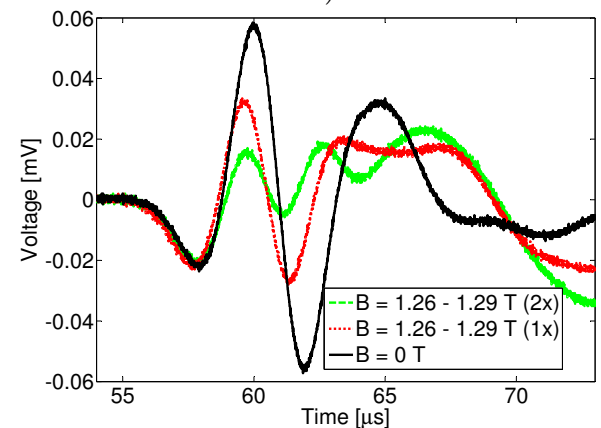

C.)

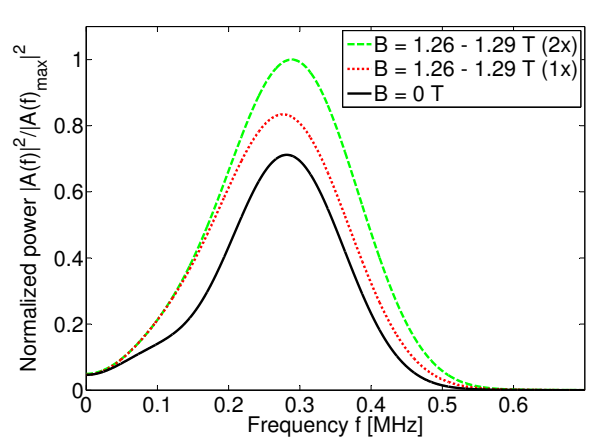

B.)

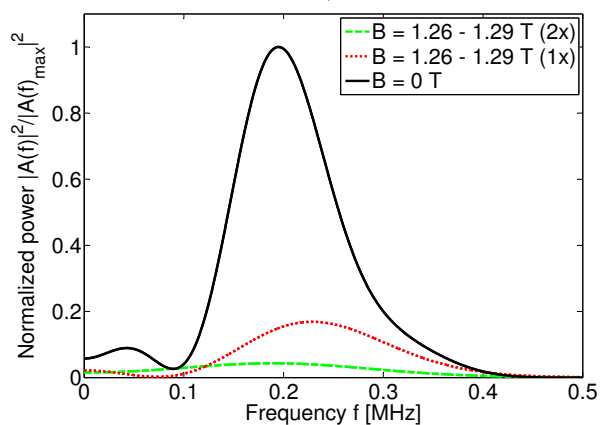

D.)

Figure 7.10: Coherent Part of the received time signals (A.,C.) transmitted through only MRF and MRF-saturated sintered sample with corresponding normalized power spectra (B.,D.). The piezo-electric acoustic transmitter is excited with a sinus-burst-signal at $2.25 \mathrm{MHz}$. 


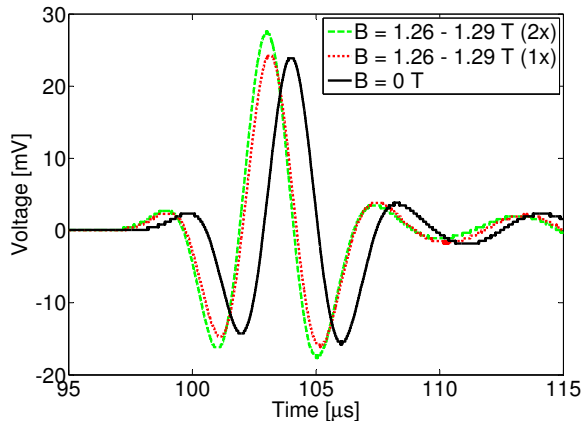

A.)

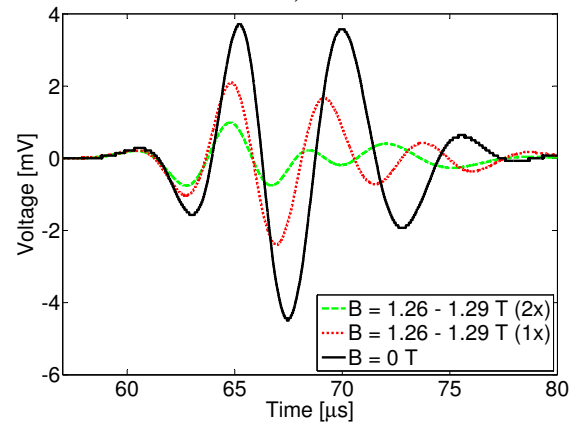

C.)

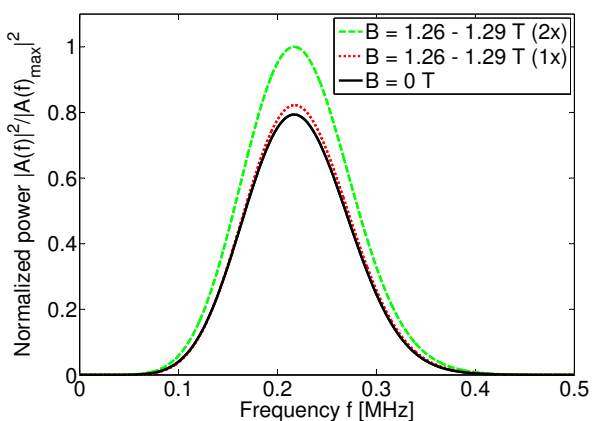

B.)

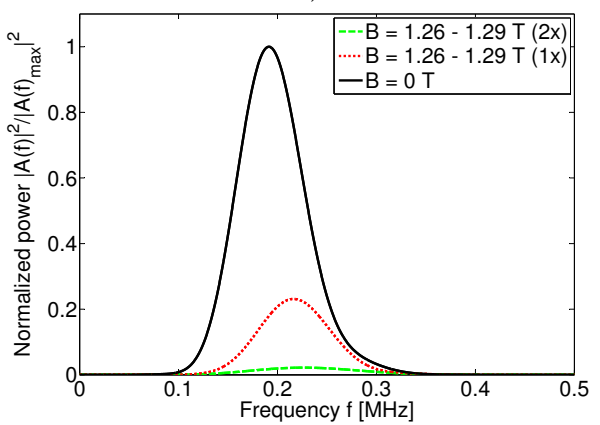

D.)

Figure 7.11: Coherent Part of the received time signals (A.,C.) transmitted through only MRF and MRF-saturated sintered sample with corresponding normalized power spectra (B.,D.). The piezo-electric acoustic transmitter is excited with a Ricker wavelet at $0.2 \mathrm{MHz}$. 


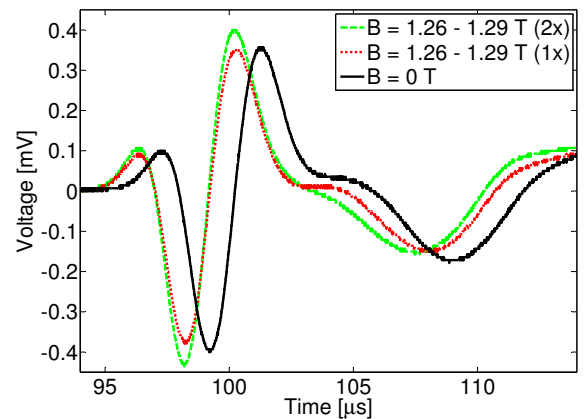

A.)

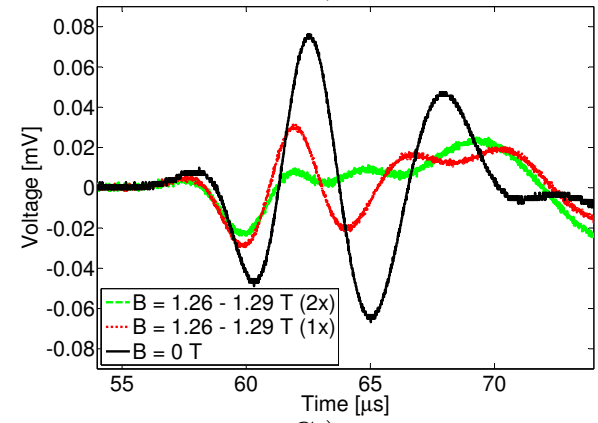

C.)

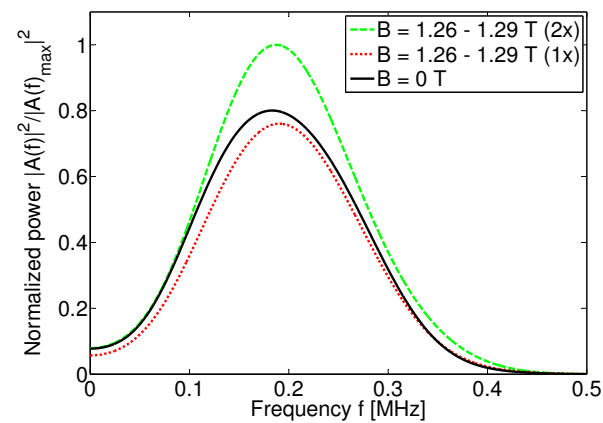

B.)

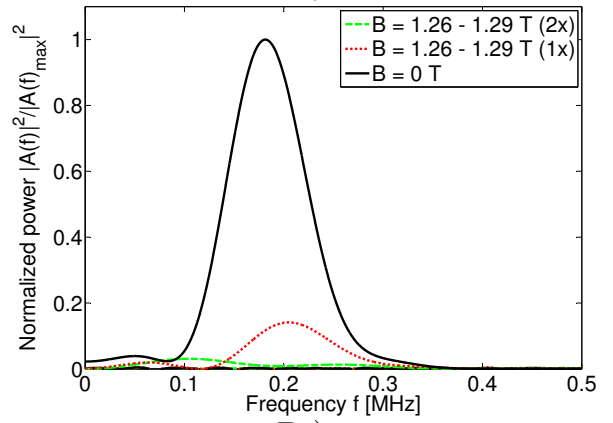

D.)

Figure 7.12: Coherent Part of the received time signals (A.,C.) transmitted through only MRF and MRF-saturated sintered sample with corresponding normalized power spectra (B.,D.). The piezo-electric acoustic transmitter is excited with a Ricker wavelet at $1.0 \mathrm{MHz}$.

magnets. Conversely, the power spectra of sample measurements show an enhancement of the filter effect, when the MRF-saturated sample is subjected to a magnetic field.

\subsubsection{Dispersion properties}

Figures 7.13 and 7.14 show the dispersion parameters for different input waveforms determined from the spectral ratios of the reference and sample measurements. The approximate frequency spectrum of the analyzed coherent wave part is represented by the gray-shaded area. As can be seen from Figure 7.13 and 7.14 the investigated sintered sample show higher (specific) attenuation effects, when the MRF-saturated sample is exposed to higher magnetic field strengths. 
This finding has been also confirmed in the normalized power spectra of the MRF-saturated sample shown in the previous section.

The measured specific attenuation $1 / Q_{P 1}(f)$, shown in Figure 7.13 (A.,B.) and 7.14 (A.,B.) steadily decreases up to a certain frequency value and finally remains relatively constant in the relevant frequency range independent from the used input signals and excitation signals. In contrast to that, the attenuation $\alpha_{P 1}(f)$ steadily increases with increasing frequency in the relevant frequency range, when the transmitter is excited with a sinus-burst-signal. However, the increase of the attenuation is not significant for higher magnetic strengths, when the Ricker wavelet is used as input signal.

Independent from the used input signal, the phase velocity, illustrated in Figure 7.13 (E.,F.) and 7.14 (E.,F.), determined at different magnetic strength fluctuate around the constant reference values, listed in Table 7.3. The fluctuations are reduced with increasing frequency. However, the fluctuations differ with the used input signal, excitation frequency and magnetic strength.

\subsection{Conclusion}

In summary, we have demonstrated that the dispersion properties of a MRFsaturated porous sintered glass bead sample can be influenced, when a magnetic field is generated. We have presented a new thin-walled measuring cell, which has enabled us to investigated the influence of an magnetic field on the acoustical wave propagation in MRF-saturated porous media. This work provides insight and experimental evidence of the influence of the pore fluid on the acoustical wave propagation in porous media. We have demonstrated that the detected and analyzed coherent wave part, which represents the fast P1-wave in Biot's theory, is attenuated, when a magnetic field is applied. The TOF remained almost constant. The reference measurements without a porous sample showed surprisingly the opposite effect. A clear signal enhancement and time shift of the coherent wave part could be determined, when the MRF is subjected to a magnetic field. In general, we have proved that the interaction between pore fluid and solid matrix can be selectively controlled by the magnetic strength, when a MRF is used as pore fluid.

\subsection{Acknowledgement}

We acknowledge support from the research program of the Stichting voor Fundamenteel Onderzoek der Materie (FOM), which is financially supported by the 


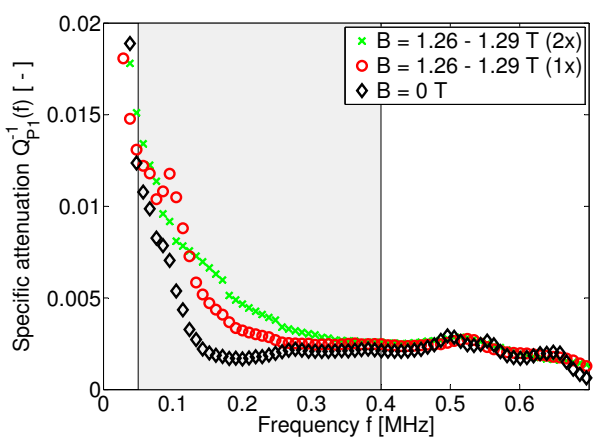

A.)

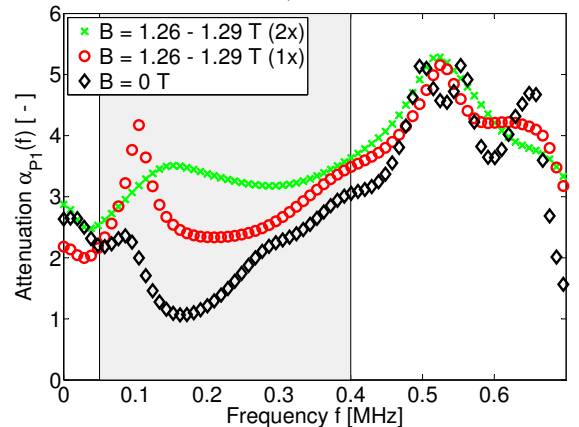

C.)

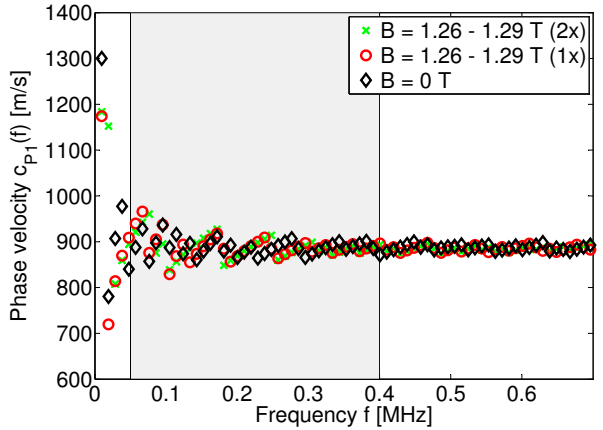

E.)

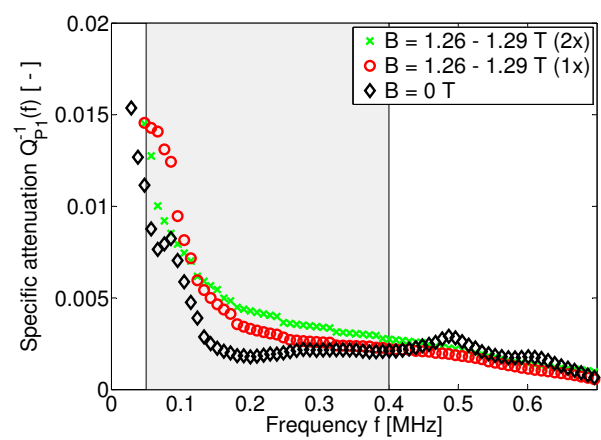

B.)

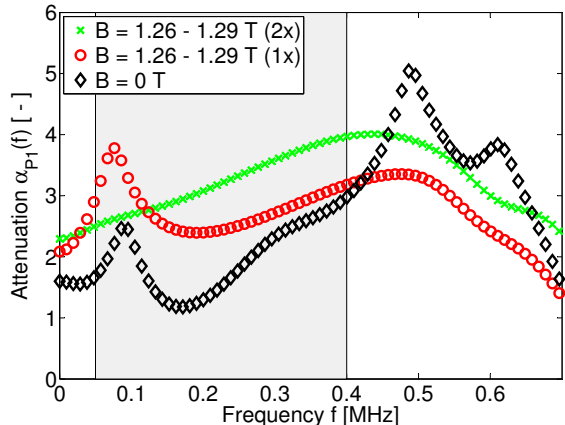

D.)

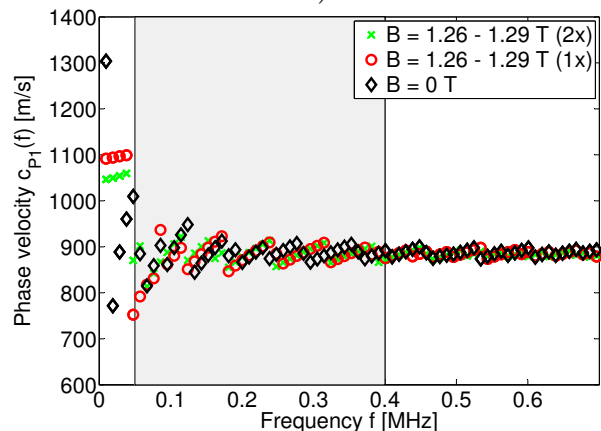

F.)

Figure 7.13: Specific attenuation $1 / Q(f)$, attenuation $\alpha(f)$ and phase velocity $c_{P h}(f)$ in dependence on the frequency of the detected fast P1-wave, where different magnetic strength $B$ are applied during ultrasound experiments. The piezo-electric transmitter is excited with a sinus-burst-signal at 0.1 (A.,C.,E.) and $1.0 \mathrm{MHz}$ (B.,D.,F.). 


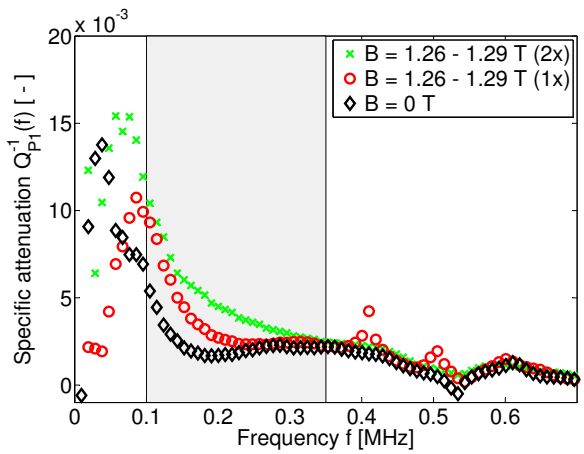

A.)

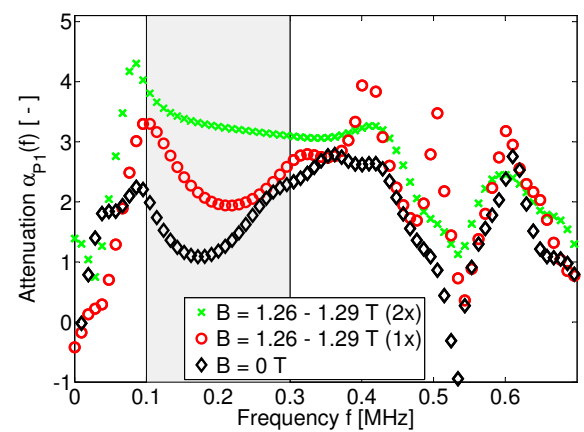

C.)

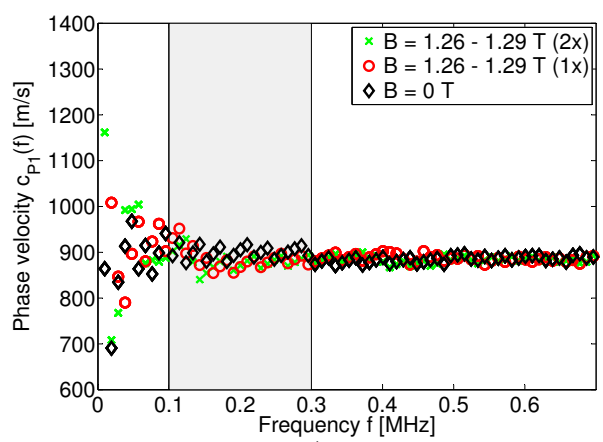

E.)

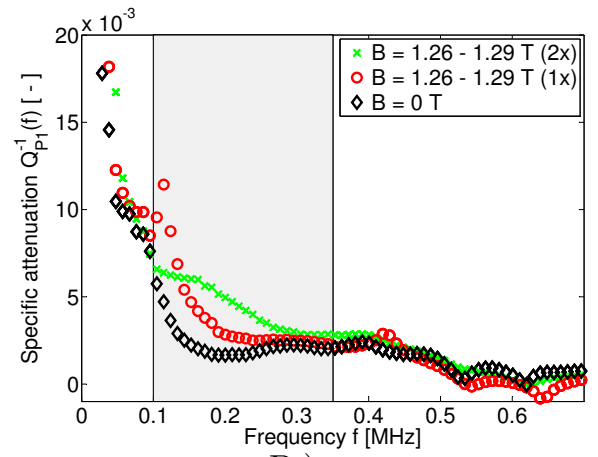

B.)

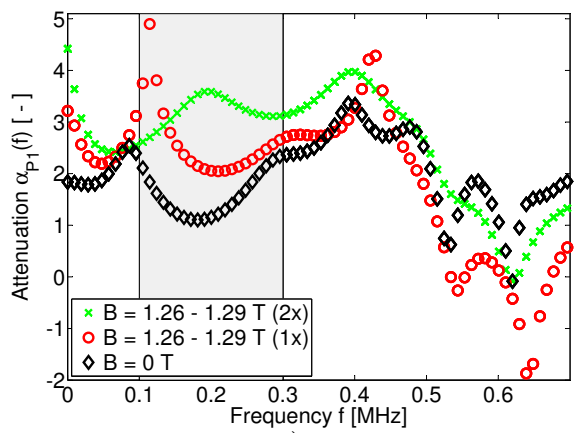

D.)

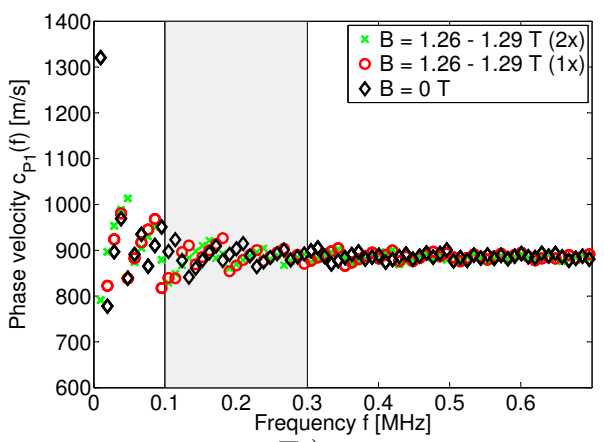

F.)

Figure 7.14: Specific attenuation $1 / Q(f)$, attenuation $\alpha(f)$ and phase velocity $c_{P h}(f)$ in dependence on the frequency of the detected fast P1-wave, where different magnetic strength $B$ are applied during ultrasound experiments. The piezo-electric transmitter is excited with a Ricker wavelet at 0.2 (A.,C.,E.) and 1.0 $\mathrm{MHz}$ (B.,D.,F.). 
"Nederlandse Organisatie voor Wetenschappelijk Onderzoek" (NWO), (project number: 09iPOG14-2). 


\section{CHAPTER 8}

\section{Summary, conclusion and Outlook}

\subsection{Summary and conclusion}

The main objectives of this investigation are the hydraulical transport and acoustical wave properties of sintered glass bead packings. The introductory chapter has provided a background information and motivation for both topics and illustrated the fundamentals of transport and wave behavior in porous media.

In the following paragraphs the main findings of each chapter are summarized and concluded with further comments on limitations and future work.

- Chapter 2 emphasized the sintering procedure and hydraulical properties the produced sintered glass bead samples. Moreover, an extensive procedure for the determination of parameters from $\mu \mathrm{CT}$ data, which highly affect the transport properties of the sintered packings, is presented and was applied to various porous samples. A good agreement between experimentally observed and numerically predicted permeabilities could be achieved for various sintered samples with different microstructures due to the used glass bead sizes and different sintering treatments. An appropriate filtering procedure of the image data revealed that the intrinsic permeability of the sintered granular packings is quantitatively and qualitatively well described by the Kozeny-Carman model. The empirical constant was determined at $c_{1}=131$ i.e. considerably smaller compared to commonly used value for monodisperse and non-sintered spherical packings of $c_{1}=18 \pi^{2}$. The numerical LB results showed that the Kozeny Carman factor not only depends on the porosity, but also on the microscopic pore structure, like 
pore throats, and particle shapes, orientations and sizes. Different segmentation procedures applied on to the data sets of different samples revealed that the porosity across the cross-sectional area of the cylindrical samples remained almost constant, while a clear porosity gradient across the sample height exists due to gravitational forces acting on the beads during sintering. Rotational sintering may eventually lead to more homogenous samples. A clear linear correlation between porosity, pore throat diameter and intrinsic permeability could be detected. The local intrinsic permeability as well as the porosity decrease linearly with increasing averaged equivalent pore throat diameter determined from the pore throat distributions of the investigated subsets. The pore throat distributions showed two distinct peaks independently from the edge sizes of the subsets, which can be clearly assigned to typical pore throat areas from three or four particle constellations.

- In Chapter 3 an innovative and elaborate set-up for the investigation of the frequency-dependent hydraulical properties of the sintered samples is presented. Different methods and set-up configurations are tested to determine the frequency-dependent permeability. A clear decrease of the dynamic permeability with increasing frequency of the induced oscillatory fluid flow could be determined for the sintered samples, when the measurement data were evaluated according to [161]. However the inertial effects leading to lower permeabilities occurred at much lower frequencies than theoretically predicted. Moreover, the pressure pore oscillation allowed to determine besides the permeability also the storage capacity and diffusivity of the samples. However, the correct choice of the frequency and amplitude remain challenging. Technical improvements of the experimental set-up, for instance the oscillation of the latex membrane, the pressure measurement as well as the volume of the downstream reservoir may eventually lead to reliable results.

- Chapter 4 dealed with the acoustical wave propagation of water-saturated sintered glass bead samples. In this chapter only the coherent wave part, which arrives at the leading edge and is characterized by large amplitudes, were considered. The coherent wave part was analyzed for different samples, which exhibit different microstructures, and used for the determination of the dispersion properties. The increasing lowpass-filter effect of the samples with increasing bead sizes and sintering durations was shown. Further properties determining the acoustical properties, such as the contact area between the beads were visualized and analyzed from $\mu \mathrm{CT}$ data and used as input parameters in Biot's theory. The coherent wave part of the received signals was confirmed in Biot's theory as the fast P1-wave. 
- While the previous chapter addressed the coherent wave part, where the wavelengths are usually greater than the glass particles sizes, chapter 5 focused on incoherent wave part, which consists usually of (multiple-) scattered waves and exhibit wavelengths in the order or smaller than glass particles. The incoherent wave part is successfully approximated by a diffusive wave model and the diffusion coefficient and quality factor are determined for a selective input signal with specific frequency.

- Chapter 6 focused on the evidence of the slow P2-wave in fluid-saturated sintered glass bead samples. While the slow P2-wave was clearly observed in water-saturated sintered sample, it's propagation in silicone oil-saturated samples is hampered due to the higher viscous coupling of the pore fluid with the solid matrix. The Biot theory was used to classify the detected signals. The attenuation of the slow P2-wave is found to be considerably higher compared to the fast P1-wave, since it's generation is connected with a higher energy dissipation due to the out-of-phase movement between the fluid and solid phase. The propagation of the slow P2-wave is additionally attenuated or even hampered through the densification of the sample in the wave propagation direction

- The study of wave propagation in MRF-saturated sintered sample in Chapter 7 revealed that the pore fluid plays an important role for the wave propagation in fluid-saturated porous media. The experiments showed that the dispersion properties of the MRF-saturated sample changes, when a magnetic field is applied. A clear increase of the frequency-dependent attenuation coefficient could be determined, when an external magnetic field was generated. Moreover, changes in the arrival times of the detected fast P1waves could be observed for the reference measurements with increasing magnetic strengths, when no sample was placed between the transducers, whereas the arrival times of the P1-waves of the sample measurements remained almost constant.

Complementary, to the quantitative analysis the main findings of this thesis can be summarized as:

\section{Transport properties:}

- The transport properties in sintered granular systems, such as the intrinsic permeability, correlate with the microscopic properties such as the averaged equivalent pore throat diameter $\left(k_{z}^{s} \propto d_{p t}\right)$ or particle arrangement $\left(c_{1}=\right.$ 131).

- An appropriate filtering procedure based on the minimization of the intraclass variance and maximization of interclass variance of the gray-scale 
value distributions during XRCT data processing is crucial for a proper segmentation and thus for the accurate determination of the permeabilities. In this respect, the applied "Delineate"- and "Median"-filter showed satisfactory results.

- New universally applicable strategies for the determination of parameters as shown for pore throats or contact areas, which affect not only the transport but also the wave properties, from $\mu \mathrm{XRCT}$ data are important for the understanding of micro-macro relationships.

- For accurate and reliable permeability experiments, a proper design and development of a measuring cell as well as the method of measurement, which include for instance the proper affixing and optimal saturation of the sample, is of great importance.

\section{Wave properties:}

- Individual phenomena, like particle size, pore fluid, magnetic strength, sintering treatment have great influence on the wave properties and decide for instance whether scattering occurs or not.

- The detection of the slow P2-wave in sintered glass bead systems requires the proper choice of glass beads, sintering treatment and pore fluid. Longer sintering duration and larger glass beads lead to stronger densification of the sample and scattering of ultrasonic waves, which makes it difficult to detect the slow P2-wave. The propagation of slow P2-wave occurs in directions, where fluid flow is possible.

- The received signals and dispersion properties are not only affected by the properties of the fluid and porous solid matrix, but also depend on the input signals used to stimulate the transmitter. This point is not considered in the Biot theory.

- The classical bi-phase Biot theory is sufficient to predict the arrival times of the detected waves, but insufficient to describe the dispersion properties, for instance, the frequency-dependent attenuation from ultrasound experiments differs qualitatively and quantitatively considerably from the theoretical predicted attenuation coefficients obtained from Biot theory.

In this thesis a conclusion for each chapter was provided. Moreover, a lot of experimental and numerical data was provided, which served to better understand the transport and wave phenomena in sintered glass bead systems and to develop new or complement already existing models. The developed experimental set-ups presented in this work have a broad generality for the investigation of transport 
and wave properties of porous media with applicability in many industrial processes. The present work also offers theoretical and technical fundamentals of processing $\mu \mathrm{CT}$ data in porous media for a wide readership. This work provides a further step in understanding the transport and wave propagation phenomena in sintered granular systems.

\subsection{Outlook}

Although several aspects of transport and wave propagation phenomena through sintered glass bead systems were treated, several remarks are given, that have to be improved or examined more deeply. From the author's point of view the subsequent issues with scientific and industrial relevance are:

- Extension of LB simulations towards oscillatory fluid flow, whereby the binarized XRCT data of the sintered specimens are used as geometrical boundary conditions, cf. [8, 143].

- Testing of further $\mu \mathrm{CT}$-based methods for the determination of pore throat sizes as shown in [177], for instance, it remains to be ascertained whether the area or the extent of the pore throat is more decisive for the intrinsic permeability.

- Solving numerically and theoretically the differential equations formulated in chapter 3 for the pressure pore oscillation method, which takes the inertial effects at high frequencies into account.

- Technical improvements of the experimental set-up for dynamic permeability measurements, such as the excitation of the latex membrane with accurately controllable amplitudes for the generation of oscillatory fluid movement or the additional recording of further measurable quantities, like the volumetrical flux, are required. This lead to more accurate results and the determination of the volumetrical flux can be additionally used to verify the results. measurements.

- Extension of dynamic hydraulic measurements towards electrokinetic methods, cf. the determination of the electrokinetic coupling coefficient $[66,67$, 181].

- Extension of ultrasound experiments towards electroseismic measurements $[146,155,156,199]$.

The above listed topics provide many interesting possible directions for future research. However, it cannot be a complete list. Although this study constitutes an 
important step towards a better understanding of the transport and wave propagation phenomena in general, and in sintered granular systems specifically, the combination of mechanical and electromagnetic phenomena in saturated sintered systems can motivate and inspire much further research. 


\section{Acknowledgement}

The present thesis is the outcome of my research as $\mathrm{PhD}$ student at the University of Twente in Enschede (The Netherlands) and the Ruhr-University in Bochum (Germany) from 2012 to 2016 . Working over four years intensely on this research and the strong collaboration between two universities and countries gave me new insights and memories.

First, I thank my supervisors Prof. Dr. Stefan Luding and Prof. Dr. Holger Steeb, who gave me this opportunity. I thank both for their professional and personal advice, the fruitful talks and the exchange of ideas.

I also thank Prof. Dr. Jens Harting and Stefan Frijters for the great and uncomplicated support for helping me with the Lattice Boltzmann simulations.

Then, I thank all the members of the chair of Continuum Mechanics and the MSM group for the support given in any form and the nice working atmosphere.

Since this work was part of the research programme of the Stichting voor Fundamenteel Onderzoek der Materie (FOM), financially supported by the Nederlandse Organisatie voor Wetenschappelijk Onderzoek (NWO) and the Stichting Shell Research, I thank all these organizations for their financial support (project number 09iPOG14-2), and appreciate the discussions during FOM-Shell progress meetings in Rijswijk.

Finally, I thank all my friends and family, who supported me in this exciting, but sometimes challenging and difficult time.

Bochum, June 2016

Ibrahim Güven 


\section{Bibliography}

[1] Silicone oil, technical data sheet - X-160-A. Technical report, Fragol GmbH \& CO. KG, October 2005.

[2] Experimental evidence for slow compressional waves. Journal of Engineering Mechanics, 131(9):908-917, 2005.

[3] Lord technical data sheet - MRF-140CG, Magneto-Rhological Fluid. Technical report, Lord Mid-Atlantic Rubber Co., June 2008.

[4] Panametrics - Ultrasonic Transducers - Wedges, cables, test blocks. Technical report, Olympus corporation, 2010.

[5] NeFeB permanent magnet, technical data sheet - Q-60-30-15N. Technical report, www.supermagnete.de, November 2011.

[6] Glass beads Muehlmeier, technical data sheet - Minibeads. Technical report, Muehlmeier GmbH \& CO. KG, September 2014.

[7] V. O. Abramov, A. V. Abramova, V. M. Bayazitov, A. V. Marnosov, S. P. Kuleshov, and A. S. Gerasin. Selective ultrasonic treatment of perforation zones in horizontal oil wells for water cut reduction. Applied Acoustics, 103, Part B:214 - 220, 2016. Ultrasound: A tool of never ending applications.

[8] P. Adler and A. Pazdniakou. Dynamic permeability of porous media by the lattice boltzmann method. In EGU General Assembly Conference Abstracts, volume 14, page 2317, 2012.

[9] M. Alderliesten. Mean particle diameters: From statistical definition to physical understanding. PhD thesis, TU Delft, Delft University of Technology, 2008.

[10] M. Alderliesten. Mean particle diameters: From statistical definition to physical understanding. Journal of Biopharmaceutical Statistics, 15(2):295$325,2005$. 
[11] A. Alghamdi and A. Olabi. Novel design concept of magneto rheological damper in squeeze mode. In International Conference on Experimental Mechanics, volume 2607, 2012.

[12] E. Alhomadhi, M. Amro, and M. Almobarky. Experimental application of ultrasound waves to improved oil recovery during waterflooding. Journal of King Saud University - Engineering Sciences, 26(1):103 - 110, 2014.

[13] C. C. Anderson, K. R. Marutyan, M. R. Holland, K. A. Wear, and J. G. Miller. Interference between wave modes may contribute to the apparent negative dispersion observed in cancellous bone. The Journal of the Acoustical Society of America, 124(3):1781-1789, 2008.

[14] K. Attenborough. Acoustical characteristics of rigid fibrous absorbents and granular materials. The Journal of the Acoustical Society of America, 73 (3):785-799, 1983.

[15] A. Q. Bauer, K. R. Marutyan, M. R. Holland, and J. G. Miller. Negative dispersion in bone: The role of interference in measurements of the apparent phase velocity of two temporally overlapping signals. The Journal of the Acoustical Society of America, 123(4):2407-2414, 2008.

[16] J. Bear and A. Verruijt. Theory and applications of transport in porous media. Springer, Dordrecht, 1987.

[17] Bear, Jacob and Verruijt, Arnold. Modeling groundwater flow and pollution, volume 2. Springer Science \& Business Media, 1987.

[18] I. A. Beresnev and P. A. Johnson. Elastic-wave stimulation of oil production: A review of methods and results. Geophysics, 59(6):1000-1017, 1994.

[19] Y. Bernabé, U. Mok, B. Evans, and F. Herrmann. Permeability and storativity of binary mixtures of high-and low-permeability materials. Journal of Geophysical Research: Solid Earth, 109(B12), 2004.

[20] Y. Bernabé, U. Mok, and B. Evans. A note on the oscillating flow method for measuring rock permeability. International Journal of Rock Mechanics and Mining Sciences, 43(2):311-316, 2006.

[21] J. G. Berryman. Confirmation of Biot's theory. Applied Physics Letters, 37 (4):382-384, 1980.

[22] B. P. Binks. Particles as surfactants - similarities and differences. Current Opinion in Colloid \& Interface Science, 7(1):21-41, 2002. 
[23] B. P. Binks and T. S. Horozov. Colloidal particles at liquid interfaces. Cambridge University Press, 2006.

[24] M. A. Biot. Theory of propagation of elastic waves in a fluid-saturated porous solid. i. low-frequency range. The Journal of the Acoustical Society of America, 28(2):168-178, 1956.

[25] M. A. Biot. Theory of propagation of elastic waves in a fluid-saturated porous solid. ii. higher frequency range. The Journal of the Acoustical Society of America, 28(2):179-191, 1956.

[26] M. A. Biot and D. G. Willis. The elastic coefficients of the theory of consolidation. Journal of Applied Mechanics, 24:594-601, 1957.

[27] M. Biot. Generalized theory of acoustic propagation in porous dissipative media. The Journal of the Acoustical Society of America, 34(9A):1254$1264,1962$.

[28] J. Bolton and E. Green. Normal incidence sound transmission through double-panel systems lined with relatively stiff, partially reticulated polyurethane foam. Applied Acoustics, 39(1-2):23-51, 1993.

[29] K. Boomsma and D. Poulikakos. The effects of compression and pore size variations on the liquid flow chracteristics in metal foams. Journal of Fluid Engineering, 124:263-272, March 2002.

[30] F. A. Boyle and N. P. Chotiros. Experimental detection of a slow acoustic wave in sediment at shallow grazing angles. The Journal of the Acoustical Society of America, 89(4B):1982-1982, 1991.

[31] W. Brace, J. Walsh, and W. Frangos. Permeability of granite under high pressure. Journal of Geophysical Research, 73(6):2225-2236, 1968.

[32] A. Buades, B. Coll, and J.-M. Morel. A non-local algorithm for image denoising. In Computer Vision and Pattern Recognition, 2005. CVPR 2005. IEEE Computer Society Conference on, volume 2, pages 60-65. IEEE, 2005.

[33] A. Buades, B. Coll, and J.-M. Morel. A review of image denoising algorithms, with a new one. Multiscale Modeling \& Simulation, 4(2):490-530, 2005.

[34] T. Butz. Fouriertransformation für Fußgänger. Springer Science \& Business Media, Teubner Verlag/ GWV Fachverlage GmbH, Wiesbaden, 4. edition, 2005. 
[35] M. T. Cantisano. Relative permeability in a shale formation in colombia using digital rock physics. Unconventional Resources Technology Conference (URTEC), 2013.

[36] L. Cardoso, F. Teboul, L. Sedel, C. Oddou, and A. Meunier. In vitro acoustic waves propagation in human and bovine cancellous bone. Journal of Bone and Mineral Research, 18(10):1803-1812, 2003.

[37] J. D. Carlson, M. J. Chrzan, and F. O. James. Magnetorheological fluid devices, February 8 1994. US Patent 5,284,330.

[38] J. D. Carlson, M. J. Chrzan, and F. O. James. Magnetorheological fluid devices, March 21 1995. US Patent 5,398,917.

[39] J. D. Carlson, D. Catanzarite, and K. St. Clair. Commercial magnetorheological fluid devices. International Journal of Modern Physics B, 10 (23n24):2857-2865, 1996.

[40] P. Carman. Fluid flow through a granular bed. Transactions of the Institutions of Chemical Engineers, 15:150-167, 1937.

[41] W. D. Carrier. Goodbye, Hazen; Hello, Kozeny-Carman. Journal of Geotechnical and Geoenvironmental Engineering, 129:1054-1056, 2003.

[42] H. Carslaw and J. Jaeger. Conduction of Heat in Solids. Oxford science publications. Clarendon Press, Oxford, 1986.

[43] S. Chaffar, F. Padilla, G. Berger, and P. Laugier. In vitro measurement of the frequency-dependent attenuation in cancellous bone between 0.2 and 2 mhz. The Journal of the Acoustical Society of America, 108(3):1281-1289, 2000 .

[44] E. Charlaix, A. Kushnick, and J. Stokes. Experimental study of dynamic permeability in porous media. Physical Review Letters, 61(14):1595, 1988.

[45] J. Claracq, J. Sarrazin, and J.-P. Montfort. Viscoelastic properties of magnetorheological fluids. Rheologica Acta, 43(1):38-49, 2003.

[46] M. Cowan, I. Jones, J. Page, and D. Weitz. Diffusing acoustic wave spectroscopy. Physical Review E, 65(6):066605, 2002.

[47] S. Cowin and L. Cardoso. Difficulties arising from different definitions of tortuosity for wave propagation in saturated poroelastic media models. ZAMM - Journal of Applied Mathematics and Mechanics / Zeitschrift für Angewandte Mathematik und Mechanik, 94(9):694-704, 2014. 
[48] F. Deroo, J.-Y. Kim, J. Qu, K. Sabra, and L. J. Jacobs. Detection of damage in concrete using diffuse ultrasound. The Journal of the Acoustical Society of America, 127(6):3315-3318, 2010.

[49] D. d'Humières, I. Ginzburg, M. Krafczyk, P. Lallemand, and L.-S. Luo. Multiple-relaxation-time lattice Boltzmann models in three dimensions. Phil. Trans. R. Soc. Lond. A, 360:437, 2002.

[50] J. P. Du Plessis and S. Woudberg. Pore scale derivation of the Ergun equation to enhance its adaptability and generalization. Chemical Engineering Science, 63:2576-2686, February 2008.

[51] A. Duda, Z. Koza, and M. Matyka. Hydraulic tortuosity in arbitrary porous media flow. Physical Review E, 84:036319, Sep 2011.

[52] N. Dukhan and C. A. Minjeur II. A two-permeability approach for assessing flow properties in metal foam. Journal of Porous Materials, 18(4):417-424, 2011.

[53] F. A. Dullien. Porous media: fluid transport and pore structure. Academic Press, Inc., San Diego, 2012.

[54] S. FEI Visualization Sciences Group. AVIZO 8.0 Reference Guide. VSG, SAS, 2013.

[55] G. Fischer. The determination of permeability and storage capacity: Pore pressure oscillation method. International Geophysics Series, 51:187-187, 1992.

[56] G. Fischer and M. Paterson. Measurement of permeability and storage capacity in rocks during deformation at high temperature and pressure. International Geophysics, 51:213-252, 1992.

[57] J. Frenkel. On the theory of seismic and seismoelectric phenomena in a moist soil. Journal of Engineering Mechanics, 131(9):879-887, 2005.

[58] S. Frijters. Liquid-liquid and liquid-solid interactions at the mesoscale. PhD thesis, Technical University of Eindhoven, Eindhoven, AZ 5612, Not published yet.

[59] S. Frijters and J. Harting. Self-assembled porous media from particlestabilized emulsions. arXiv preprint arXiv:1408.2974, 2014.

[60] G. Gaiselmann, M. Neumann, V. Schmidt, O. Pecho, T. Hocker, and L. Holzer. Quantitative relationships between microstructure and effective transport properties based on virtual materials testing. American Institute of Chemical Engineers Journal, 60(6):1983-1999, 2014. 
[61] F. Gassmann. Über die Elastizität poröser Medien. Vierteljahrschrift der Naturforschenden Gesellschaft in Zürich, 96:1-23, 1951.

[62] S. Genc and P. P. Phulé. Rheological properties of magnetorheological fluids. Smart Materials and Structures, 11(1):140, 2002.

[63] A. Gholamy and V. Kreinovich. Why ricker wavelets are successful in processing seismic data: Towards a theoretical explanation. In Computational Intelligence for Engineering Solutions (CIES), 2014 IEEE Symposium on, pages 11-16. IEEE, 2014.

[64] J. Ginder, L. Davis, and L. Elie. Rheology of magnetorheological fluids: Models and measurements. International Journal of Modern Physics B, 10 (23n24):3293-3303, 1996.

[65] J. M. Ginder. Behavior of magnetorheological fluids. Mrs Bulletin, 23(08): 26-29, 1998.

[66] P. W. Glover and N. Déry. Streaming potential coupling coefficient of quartz glass bead packs: Dependence on grain diameter, pore size, and pore throat radius. Geophysics, 75(6):F225-F241, 2010.

[67] P. Glover and E. Walker. Grain-size to effective pore-size transformation derived from electrokinetic theory. Geophysics, 74(1):E17-E29, 2008.

[68] S. Griffiths, A. Rescaglio, and F. Melo. Ultrasound propagation in wet and airless non-consolidated granular materials. Ultrasonics, 50(2):139$144,2010$.

[69] I. Gueven, S. Frijters, S. Luding, H. J., and H. Steeb. Hydraulical Properties of porous sintered glass bead systems. Granular Matter. submitted 2016.

[70] I. Gueven, P. Kurzeja, S. Luding, and H. Steeb. Experimental evaluation of phase velocities and tortuosity in fluid saturated highly porous media. Proceedings in Applied Mathematics and Mechanics, 12(1):401-402, 2012.

[71] I. Gueven, S. Luding, and H. Steeb. Hydraulic and acoustic investigation of sintered glass beads. AIP Conference Proceedings, 1542(1):581-584, 2013.

[72] A. Gusmeroli, R. A. Clark, T. Murray, A. D. Booth, B. Kulessa, and B. E. Barrett. Seismic wave attenuation in the uppermost glacier ice of storglaciaren, sweden. Journal of Glaciology, 56(196):249-256, 2010.

[73] G. Haiat, F. Padilla, M. Svrcekova, Y. Chevalier, D. Pahr, F. Peyrin, P. Laugier, and P. Zysset. Relationship between ultrasonic parameters and apparent trabecular bone elastic modulus: a numerical approach. Journal of Biomechanics, 42(13):2033-2039, 2009. 
[74] T. Haire and C. Langton. Biot theory: a review of its application to ultrasound propagation through cancellous bone. Bone, 24(4):291-295, 1999.

[75] M. Halisch. Application and Assessment of the Lattice Boltzmann Method for Fluid Flow Modeling in Porous Rocks. PhD thesis, 2013.

[76] M. Halisch. The REV challenge-estimating representative elementary volumes and porous rock inhomogeneity from high resolution micro-CT data sets. SCA2013-069, September 2013.

[77] M. Halisch, E. Vogt, C. Müller, A. Cano-Odena, D. Pattyn, P. Hellebaut, and K. van der Kamp. Capillary flow porometry-assessment of an alternative method for the determination of flow relevant parameters of porous rocks. SCA2013-00\%, 2013.

[78] S. M. Hassanizadeh. Derivation of basic equations of mass transport in porous media, part 1. macroscopic balance laws. Advances in Water Resources, 9(4):196-206, 1986.

[79] M. Hecht and J. Harting. Implementation of one-site velocity boundary condition for D3Q19 lattice Boltzmann simulations. J. Stat. Mech., 10: 1742-5468, 2010.

[80] R. Hennino, N. Trégourès, N. Shapiro, L. Margerin, M. Campillo, B. Van Tiggelen, and R. Weaver. Observation of equipartition of seismic waves. Physical Review Letters, 86(15):3447, 2001.

[81] R. Hilfer. Local-porosity theory for flow in porous media. Physical Review B, 45(13):7115-7121, Apr 1992.

[82] R. Hilfer. Transport and relaxation phenomena in porous media. Advances in Chemical Physics, 92:299-424, 1996.

[83] A. Hosokawa. Simulation of ultrasound propagation through bovine cancellous bone using elastic and Biot's finite-difference time-domain methods. The Journal of the Acoustical Society of America, 118(3):1782-1789, 2005.

[84] A. Hosokawa and T. Otani. Ultrasonic wave propagation in bovine cancellous bone. The Journal of the Acoustical Society of America, 101(1): 558-562, 1997.

[85] A. Hosokawa and T. Otani. Acoustic anisotropy in bovine cancellous bone. The Journal of the Acoustical Society of America, 103(5):2718-2722, 1998. 
[86] A. Houston, W. Otten, P. Baveye, and S. Hapca. Adaptive-window indicator kriging: A thresholding method for computed tomography images of porous media. Computers and Geosciences, 54:239-248, 2013.

[87] E. R. Hughes, T. G. Leighton, G. W. Petley, P. R. White, and R. C. Chivers. Estimation of critical and viscous frequencies for Biot theory in cancellous bone. Ultrasonics, 41(5):365 - 368, 2003.

[88] E. R. Hughes, T. G. Leighton, G. W. Petley, and P. R. White. Ultrasonic propagation in cancellous bone: a new stratified model. Ultrasound in Medicine and Biology, 25(5):811 - 821, 1999.

[89] P. P. Iassonov and I. A. Beresnev. A model for enhanced fluid percolation in porous media by application of low-frequency elastic waves. Journal of Geophysical Research: Solid Earth, 108(B3), 2003.

[90] R. Jaafar and W. J. Likos. Estimating water retention characteristics of sands from grain size distribution using idealized packing conditions. Geotechnical Testing Journal, 34(5):1-14, 2011.

[91] Q. Ji, L. Le, L. Filipow, and S. Jackson. Ultrasonic wave propagation in water-saturated aluminum foams. Ultrasonics, 36(6):759 - 765, 1998.

[92] X. Jia. Codalike multiple scattering of elastic waves in dense granular media. Physical Review Letter, 93:154303, 2004.

[93] S. Job, A. Strybulevych, and J. H. Page. Ultrasonic wave transport in weakly confined granular media in the intermediate frequency regime. In S. F. d'Acoustique, editor, Acoustics 2012, Nantes, France, 2012.

[94] D. L. Johnson and T. J. Plona. Acoustic slow waves and the consolidation transition. The Journal of the Acoustical Society of America, 72(2):556-565, 1982.

[95] D. L. Johnson, J. Koplik, and R. Dashen. Theory of dynamic permeability and tortuosity in fluid-saturated porous media. Journal of Fluid Mechanics, 176:379-402, 1987.

[96] D. H. Johnston, M. N. Toksöz, and A. Timur. Attenuation of seismic waves in dry and saturated rocks: II. Mechanisms. Geophysics, 44(4):691-711, 1979.

[97] M. R. Jolly, J. W. Bender, and J. D. Carlson. Properties and applications of commercial magnetorheological fluids. Journal of Intelligent Material Systems and Structures, 10(1):5-13, 1999. 
[98] T. D. Jones. Pore fluids and frequency-dependent wave propagation in rocks. Geophysics, 51(10):1939-1953, 1986.

[99] M. Kaczmarek, J. Kubik, and M. Pakula. Short ultrasonic waves in cancellous bone. Ultrasonics, 40(1):95-100, 2002.

[100] O. Kelder. Frequency-depent wave propagation in water-saturated porous media. PhD thesis, TU Delft, Delft University of Technology, 1998.

[101] O. Kelder and D. M. Smeulders. Observation of the biot slow wave in watersaturated nivelsteiner sandstone. Geophysics, 62(6):1794-1796, 1997.

[102] R. Kranz, J. Saltzman, and J. Blacic. Hydraulic diffusivity measurements on laboratory rock samples using an oscillating pore pressure method. International Journal of Rock Mechanics and Mining Sciences and Geomechanics Abstracts, 27(5):345 - 352, 1990.

[103] H.-J. Kümpel. About the potential of wells to reflect stress variations within inhomogeneous crust. Tectonophysics, 211(1):317-336, 1992.

[104] P. S. Kurzeja and H. Steeb. About the transition frequency in biotâẮ́s theory. The Journal of the Acoustical Society of America, 131(6):EL454EL460, 2012.

[105] P. S. Kurzeja. Waves in partially saturated porous media: An investigation on multiple scales. PhD thesis, Ruhr-University Bochum, Bochum (Germany), 44801, 2014.

[106] C. M. Langton, S. B. Palmer, and R. W. Porter. The measurement of broadband ultrasonic attenuation in cancellous bone. Engineering in Medicine, 13(2):89-91, 1984.

[107] P. Laugier and G. Haiat. Bone Quantitative Ultrasound. Springer Netherlands, 2011.

[108] B. P. Lawney and S. Luding. Mass-disorder effects on the frequency filtering in one-dimensional discrete particle systems. AIP Conference Proceedings, 1542(1):535-538, 2013.

[109] B. Lawney and S. Luding. Frequency filtering in disordered granular chains. Acta Mechanica, 225(8):2385-2407, 2014.

[110] K. I. Lee and S. W. Yoon. Comparison of acoustic characteristics predicted by Biot's theory and the modified Biot-Attenborough model in cancellous bone. Journal of Biomechanics, 39(2):364-368, 2006. 
[111] K. I. Lee, H.-S. Roh, and S. W. Yoon. Acoustic wave propagation in bovine cancellous bone: application of the modified biot-attenborough model. The Journal of the Acoustical Society of America, 114(4):2284-2293, 2003.

[112] E. Lemaire, A. Meunier, G. Bossis, J. Liu, D. Felt, P. Bashtovoi, and N. Matoussevitch. Influence of the particle size on the rheology of magnetorheological fluids. Journal of Rheology, 39(5):1011-1020, 1995.

[113] D. Lesnic, L. Elliott, D. Ingham, B. Clennell, and R. Knipe. A mathematical model and numerical investigation for determining the hydraulic conductivity of rocks. International Journal of Rock Mechanics and Mining Sciences, 34(5):741 - 759, 1997.

[114] L. Lin, J. Cheng, W. Lin, and Y.-X. Qin. Prediction of trabecular bone principal structural orientation using quantitative ultrasound scanning. Journal of Biomechanics, 45(10):1790-1795, 2012.

[115] H. Liu, Q. Kang, C. R. Leonardi, S. Schmieschek, A. Narváez, B. D. Jones, J. R. Williams, A. J. Valocchi, and J. Harting. Multiphase lattice Boltzmann simulations for porous media applications. Computational Geosciences, pages 1-29, 2014.

[116] S. Ma and N. R. Morrow. Relationships between porosity and permeability for porous rocks. In International symposium of the Society of Core Analysts, September, pages 8-10, 1996.

[117] A. Manbachi, S. Hashemi, B. Lashkari, et al. On estimating the directionality distribution in pedicle trabecular bone from micro-CT images. Physiological Measurement, 35(12):2415, 2014.

[118] C. Manwart and R. Hilfer. Numerical simulation of creeping fluid flow in reconstruction models of porous media. Physica A: Statistical Mechanics and its Applications, (1):706-713, 2002.

[119] C. Manwart, U. Aaltosalmi, A. Koponen, R. Hilfer, and J. Timonen. Lattice-boltzmann and finite-difference simulations for the permeability for three-dimensional porous media. Physical Review E, 66(1):016702, 2002.

[120] K. R. Marutyan, M. R. Holland, and J. G. Miller. Anomalous negative dispersion in bone can result from the interference of fast and slow waves. The Journal of the Acoustical Society of America, 120(5):EL55-EL61, 2006.

[121] G. Mavko, T. Mukerji, and J. Dvorkin. The rock physics handbook: Tools for seismic analysis of porous media. Cambridge university press, 2009. 
[122] R. Meyer. Enhanced oil recovery using multiple sonic sources, December 8 2009. US Patent 7,628,202.

[123] R. Meyer and C. Tarnawskyj. Method for improving oil recovery using an ultrasound technique, June 18 2002. US Patent 6,405,796.

[124] M. Mohsin and M. Meribout. An extended model for ultrasonic-based enhanced oil recovery with experimental validation. Ultrasonics sonochemistry, 23:413-423, 2015.

[125] J. B. Molyneux and D. R. Schmitt. Compressional-wave velocities in attenuating media: A laboratory physical model study. Geophysics, 65(4): 1162-1167, 2000.

[126] O. Mouraille. Sound propagation in dry granular materials : discrete element simulations, theory, and experiments. $\mathrm{PhD}$ thesis, Enschede (Netherlands), 7500 AE, 2009.

[127] O. Mouraille and S. Luding. Sound waves in 3D periodic granular materials. The Journal of the Acoustical Society of America, 123(5):3146-3146, 2008.

[128] A. Nabovati, E. W. Llewellin, and A. C. Sousa. A general model for the permeability of fibrous porous media based on fluid flow simulations using the lattice boltzmann method. Composites Part A: Applied Science and Manufacturing, 40(6):860-869, 2009.

[129] A. Narváez, T. Zauner, F. Raischel, R. Hilfer, and J. Harting. Quantitative analysis of numerical estimates for the permeability of porous media from lattice-Boltzmann simulations. Journal of Statistical Mechanics: Theory and Experiment, 2010(11):P11026, 2010.

[130] A. Narváez, K. Yazdchi, S. Luding, and J. Harting. From creeping to inertial flow in porous media: a lattice Boltzmann-finite element study. Journal of Statistical Mechanics: Theory and Experiment, 2013(02):P0 2038, 2013.

[131] P. H. F. Nicholson, G. Lowet, C. M. Langton, J. Dequeker, and G. V. der Perre. A comparison of time-domain and frequency-domain approaches to ultrasonic velocity measurement in trabecular bone. Physics in Medicine and Biology, 41(11):2421, 1996.

[132] C. F. Njeh, T. Fuerst, E. Diessel, and H. K. Genant. Is quantitative ultrasound dependent on bone structure? a reflection. Osteoporosis International, 12(1):1-15, 2001. 
[133] C. Njeh, C. Boivin, and C. Langton. The role of ultrasound in the assessment of osteoporosis: a review. Osteoporosis International, 7(1):7-22, 1997.

[134] F. Padilla and P. Laugier. Phase and group velocities of fast and slow compressional waves in trabecular bone. The Journal of the Acoustical Society of America, 108(4):1949-1952, 2000.

[135] J. H. Page. Ultrasonic wave transport in strongly scattering media. Proceedings of the International School of Physics "Enrico Fermi", pages 75-93, 2011.

[136] J. H. Page, H. P. Schriemer, A. E. Bailey, and D. A. Weitz. Experimental test of the diffusion approximation for multiply scattered sound. Physical Review E, 52:3106-3114, 1995.

[137] J. H. Page, I. P. Jones, H. P. Schriemer, M. L. Cowan, P. Sheng, and D. A. Weitz. Diffusive transport of acoustic waves in strongly scattering media. Physica B, 263:37-39, 1999.

[138] J. Page, H. Schriemer, I. Jones, P. Sheng, and D. Weitz. Classical wave propagation in strongly scattering media. Physica A: Statistical Mechanics and its Applications, 241(1):64-71, 1997.

[139] J. Page, M. Cowan, P. Sheng, and D. Weitz. Diffusing acoustic wave transport and spectroscopy. In IUTAM Symposium on Mechanical and Electromagnetic Waves in Structured Media, volume 91 of Solid Mechanics and Its Applications, pages 121-132. Springer Netherlands, 2002.

[140] H. J. Pain and H. J. Pain. The physics of vibrations and waves. John Wiley, Chichester, 2005.

[141] B. J. Park, F. F. Fang, and H. J. Choi. Magnetorheology: Materials and application. Soft Matter, 6:5246-5253, 2010.

[142] J. H. Park, B. D. Chin, and O. O. Park. Rheological properties and stabilization of magnetorheological fluids in a water-in-oil emulsion. Journal of Colloid and Interface Science, 240(1):349 - 354, 2001.

[143] A. Pazdniakou and P. Adler. Dynamic permeability of porous media by the lattice boltzmann method. Advances in Water Resources, 62:292-302, 2013.

[144] T. J. Plona. Observation of a second bulk compressional wave in a porous medium at ultrasonic frequencies. Applied Physics Letters, 36(4):259-261, 1980 . 
[145] M. L. Porter and D. Wildenschild. Image analysis algorithms for estimating porous media multiphase flow variables from computed microtomography data: a validation study. Computational Geosciences, 14(1):15-30, 2010.

[146] S. R. Pride and F. Morgan. Electrokinetic dissipation induced by seismic waves. Geophysics, 56(7):914-925, 1991.

[147] S. R. Pride. Relationships between seismic and hydrological properties. In R. Yoram and S. S. Hubbard, editors, Hydrogeophysics, pages 253-290. Springer, New York, 2005.

[148] Y. H. Qian, D. d'Humières, and P. Lallemand. Lattice BGK models for Navier-Stokes equation. Europhysics Letters, 17:479, 1992.

[149] B. Quintal, R. Jänicke, J. G. Rubino, H. Steeb, and K. Holliger. Sensitivity of S-wave attenuation to the connectivity of fractures in fluid-saturated rocks. Geophysics, 79(5):WB15-WB24, 2014.

[150] J. Renner and H. Steeb. Modeling of fluid transport in geothermal research. In W. Freeden, M. Z. Nashed, and T. Sonar, editors, Handbook of Geomathematics, pages 1443-1500. Springer Berlin Heidelberg, 2015.

[151] J. R. Rice and M. P. Cleary. Some basic stress diffusion solutions for fluidsaturated elastic porous media with compressible constituents. Reviews of Geophysics and Space Physics, 14(2):227-241, 1976.

[152] W. Sachse and Y. Pao. On the determination of phase and group velocities of dispersive waves in solids. Journal of Applied Physics, 49(8):4320-4327, 1978.

[153] E. H. Saenger, F. Enzmann, Y. Keehm, and H. Steeb. Digital rock physics: Effect of fluid viscosity on effective elastic properties. Journal of Applied Geophysics, 74(4):236 - 241, 2011.

[154] E. H. Saenger, D. Uribe, R. Jänicke, O. Ruiz, and H. Steeb. Digital material laboratory : wave propagation effects in open-cell aluminium foams. International Journal of Engineering Science, 58:115-123, 2012.

[155] M. Schakel and D. Smeulders. Seismoelectric reflection and transmission at a fluid/porous-medium interface. The Journal of the Acoustical Society of America, 127(1):13-21, 2010.

[156] M. D. Schakel. Coupled seismic and electromagnetic wave propagation. $\mathrm{PhD}$ thesis, Delft University of Technology, Delft (Netherlands), 2600 AA, 2011. 
[157] R. J. Schalkoff. Digital image processing and computer vision, volume 286. Wiley New York, 1989.

[158] A. Scheuermann and A. Bieberstein. Experimental Unsaturated Soil Mechanics, chapter Determination of the Soil Water Retention Curve and the Unsaturated Hydraulic Conductivity from the Particle Size Distribution, pages 421-433. Springer Berlin Heidelberg, Berlin, Heidelberg, 2007.

[159] S. Schlüter, A. Sheppard, K. Brown, and D. Wildenschild. Image processing of multiphase images obtained via x-ray microtomography: a review. Water Resources Research, 50(4):3615-3639, 2014.

[160] F. C. Schoemaker, D. M. J. Smeulders, and E. C. Slob. Simultaneous determination of dynamic permeability and streaming potential. SEG Technical Program Expanded Abstracts, 26(1):1555-1559, 2007.

[161] F. C. Schoemaker. Electrokinetic conversion. PhD thesis, Delft University of Technology, Delft (Netherlands), 2600 AA, 2011.

[162] P. Sheng. Scattering and localization of classical waves in random media, volume 8. World Scientific Series on Directions in Condensed Matter Physics, 1990.

[163] P. Sheng. Introduction to Wave Scattering: Localization and Mesoscopic Phenomena, volume 88. Springer-Verlag Berlin, Heidelberg, 2006.

[164] P. Sheng and M.-Y. Zhou. Dynamic permeability in porous media. Physical Review Letter, 61:1591-1594, 1988.

[165] R. Sivanesapillai, H. Steeb, and A. Hartmaier. Transition of effective hydraulic properties from low to high reynolds number flow in porous media. Geophysical Research Letters, 41(14):4920-4928, 2014.

[166] D. M. J. Smeulders, R. L. G. M. Eggels, and M. E. H. van Dongen. Dynamic permeability: reformulation of theory and new experimental and numerical data. Journal of Fluid Mechanics, 245:211-227, 1992.

[167] D. M. J. Smeulders. On wave propagation in saturated and partially saturated porous media. PhD thesis, Technical University Eindhoven, Eindhoven, 5612 AZ, 1992.

[168] I. Song and J. Renner. Analysis of oscillatory fluid flow through rock samples. Geophysical Journal International, 170(1):195-204, 2007. 
[169] I. Song, A. P. Rathbun, and D. M. Saffer. Uncertainty analysis for the determination of permeability and specific storage from the pulse-transient technique. International Journal of Rock Mechanics and Mining Sciences, 64:105-111, 2013.

[170] M. A. Spaid and F. R. Phelan Jr. Lattice Boltzmann methods for modeling microscale flow in fibrous porous media. Physics of Fluids, 9(9):2468-2474, 1997.

[171] B. Spencer, S. Dyke, M. Sain, and J. Carlson. Phenomenological model for magnetorheological dampers. Journal of engineering mechanics, 123 (3):230-238, 1997.

[172] H. Steeb. Ultrasound propagation in cancellous bone. Archive of Applied Mechanics, 80(5):489-502, 2010.

[173] H. Steeb, P. Kurzeja, and S. Schmalholz. Wave propagation in unsaturated porous media. Acta Mechanica, 225(8):2435-2448, 2014.

[174] O. Stenzel, M. Salzer, V. Schmidt, P. W. Cleary, and G. W. Delaney. Quantitative structural analysis of simulated granular packings of non-spherical particles. Granular Matter, 16(4):457-468, 2014.

[175] O. Stenzel, O. Pecho, L. Holzer, M. Neumann, and V. Schmidt. Predicting effective conductivities based on geometric microstructure characteristics. American Institute of Chemical Engineers Journal, 2016. in print.

[176] R. D. Stoll. Acoustic waves in ocean sediments. Geophysics, 42(4):715-725, 1977.

[177] H. Taylor, C. O'Sullivan, and W. Sim. A new method to identify void constrictions in micro-ct images of sand. Computers and Geotechnics, 69: 279-290, 2015.

[178] H. D. To, A. Scheuermann, and D. J. Williams. A new simple model for the determination of the pore constriction size distribution. In 6th International Conference on Scour and Erosion (ICSE-6), pages 295-303. Société Hydrotechnique de France (SHF), 2012.

[179] R. Tonn. Comparison of seven methods for the computation of Q. Physics of the Earth and Planetary Interiors, 55(3):259 - 268, 1989.

[180] N. Viard and A. Derode. Experimental determination of the diffusion constant for ultrasonic waves in 2-d multiple scattering media with focused beamforming. In Acoustics 2012, 2012. 
[181] J. Vinogradov, M. Jaafar, and M. Jackson. Measurement of streaming potential coupling coefficient in sandstones saturated with natural and artificial brines at high salinity. Journal of Geophysical Research: Solid Earth, 115(B12), 2010.

[182] J. Wang and G. Meng. Magnetorheological fluid devices: principles, characteristics and applications in mechanical engineering. Proceedings of the Institution of Mechanical Engineers, Part L: Journal of Materials Design and Applications, 215(3):165-174, 2001.

[183] Y. Wang. Frequencies of the ricker wavelet. Geophysics, 80(2):A31-A37, 2015.

[184] K. Wear. A stratified model to predict dispersion in trabecular bone. $U l$ trasonics, Ferroelectrics, and Frequency Control, IEEE Transactions on, 48 (4):1079-1083, July 2001.

[185] K. Wear et al. Ultrasonic scattering from cancellous bone: a review. Ultrasonics, Ferroelectrics, and Frequency Control, IEEE Transactions on, 55 (7):1432-1441, 2008.

[186] R. Weaver. Ultrasonics in an aluminum foam. Ultrasonics, 36(1-5):435 442, 1998.

[187] R. Weaver, Y. Zhang, W. Sachse, and K. Green. Diffusive ultrasound polycrystalline solids. In Ultrasonics International 91, pages $507-510$. Butterworth-Heinemann, 1991.

[188] R. L. Weaver and W. Sachse. Diffusion of ultrasound in a glass bead slurry. The Journal of the Acoustical Society of America, 97(4):2094-2102, 1995.

[189] C. Wei and K. K. Muraleetharan. Acoustical characterization of fluidsaturated porous media with local heterogeneities: Theory and application. International Journal of Solids and Structures, 43(5):982-1008, 2006.

[190] D. Wildenschild, C. Vaz, M. Rivers, D. Rikard, and B. Christensen. Using x-ray computed tomography in hydrology: systems, resolutions, and limitations. Journal of Hydrology, 267(3âĂŞ4):285 - 297, 2002.

[191] D. Wildenschild and A. P. Sheppard. X-ray imaging and analysis techniques for quantifying pore-scale structure and processes in subsurface porous medium systems. Advances in Water Resources, 51:217 - 246, 2013.

[192] J. L. Williams. Ultrasonic wave propagation in cancellous and cortical bone: Prediction of some experimental results by biotâĂ $\mathbf{s}$ theory. The Journal of the Acoustical Society of America, 91(2):1106-1112, 1992. 
[193] K. Wilmanski. Tortuosity and objective relative accelerations in the theory of porous materials. Proceedings of the Royal Society of London A: Mathematical, Physical and Engineering Sciences, 461(2057):1533-1561, 2005.

[194] J. R. Womersley. Method for the calculation of velocity, rate of flow and viscous drag in arteries when the pressure gradient is known. The Journal of Physiology, 127(3):553-563, 1955.

[195] K. Yazdchi and S. Luding. Upscaling and microstructural analysis of the flow-structure relation perpendicular to random, parallel fiber arrays. Chemical Engineering Science, 98:173-185, 2013.

[196] K. Yazdchi. Micro-macro relations for flow through fibrous media. $\mathrm{PhD}$ thesis, University of Twente, Enschede (Netherlands), 7500 AE, 2012.

[197] S. Zhang, R. E. Klimentidis, and P. Barthelemy. Micron to millimeter upscaling of shale rock properties based on $3 \mathrm{~d}$ imaging and modeling. In International Symposium of the Society of Core Analysts, pages 27-30, 2012.

[198] R. Zheng, L. H. Le, M. D. Sacchi, D. Ta, and E. Lou. Spectral ratio method to estimate broadband ultrasound attenuation of cortical bones in vitro using multiple reflections. Physics in Medicine and Biology, 52(19): $5855,2007$.

[199] Z. Zhu, M. N. Toksöz, and D. R. Burns. Electroseismic and seismoelectric measurements of rock samples in a water tank. Geophysics, 73(5):E153E164, 2008.

[200] Q. Zou and X. He. On pressure and velocity boundary conditions for the lattice Boltzmann BGK model. Physics of Fluids, 9:1591, 1997. 



\section{Curriculum Vitae - Ibrahim GüVEN}

Place and Date of Birth: Bochum, Germany | 09 December 1983

\section{Professional Occupation}

since $01 / 2012$

Ph.D. student and research assistant

Multi Scale Mechanics Group

(Prof. Dr. rer. nat. S. Luding)

University of Twente, The Netherlands

since $01 / 2012$

Ph.D. student and research assistant

Chair of Continuum Mechanics

(Prof. Dr.-Ing. H. Steeb)

Ruhr-Universität Bochum, Germany

06/2009 - 09/2009 Internship

Research and Development Center for Gas and Steam Turbines and Generators

Siemens AG, Mühlheim an der Ruhr, Germany

10/2010 - 10/2011 Student assistant

Chair of Continuum Mechanics

(Prof. Dr.-Ing. H. Steeb)

Ruhr Universität Bochum, Germany

12/2006 - 05/2009 Student assistant

Chair of Technical Mechanics

(Prof. Em. Dr.-Ing. O.T.Bruhns)

Ruhr Universität Bochum, Germany

\section{University Education}

since 08/2012 Member of RUB Research School

09/2003 - 11/2011 Studies of Environmental Engineering and Resource

Management

Academic Degree: Diploma (Dipl. Ing.)

Ruhr Universität Bochum, Germany

\section{School Education}

06/1994 -06/2003 Higher School Certificate (Abitur)

Lessing Gymnasium, Bochum, Germany 


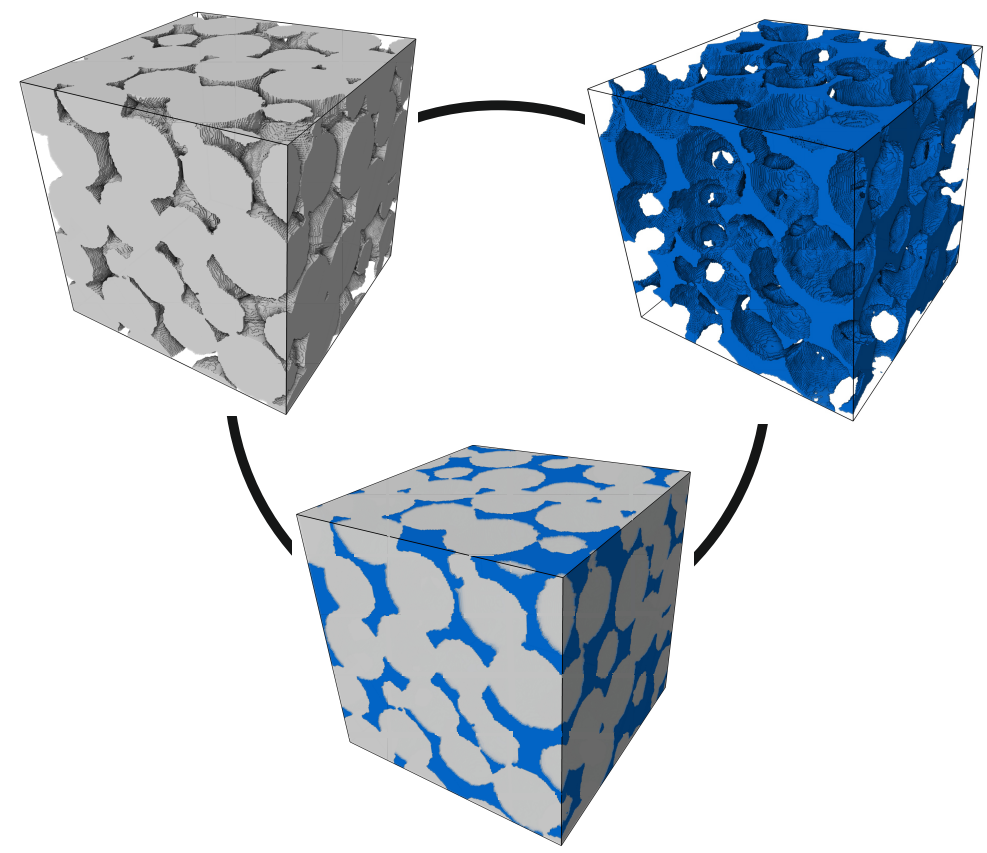

(n) 